\title{
Supporting Information for: \\ Investigation of Stepwise Covalent Synthesis on a Surface Yielding Porphyrin-Based Multicomponent Architectures
}

Izabela Schmidt, Jieying Jiao, Patchanita Thamyongkit, Duddu S. Sharada, David F. Bocian, and Jonathan S. Lindsey

\section{Table of Contents}

I. General Experimental Section S1

II. Noncommercial Compounds S1

III. Experimental Procedures S1-S7

IV. Alternative Synthesis of Porphyrin $42 \quad$ S8-S9

V. Characterization Data for Selected Compounds S10-S109

\section{General Experimental Section.}

All ${ }^{1} \mathrm{H}$ NMR spectra $(400 \mathrm{MHz})$ and ${ }^{13} \mathrm{C}$ NMR spectra $(100 \mathrm{MHz})$ were collected in $\mathrm{CDCl}_{3}$ unless noted otherwise. Absorption spectra were collected in $\mathrm{CH}_{2} \mathrm{Cl}_{2}$ at room temperature unless noted otherwise. Mass spectra of porphyrins were obtained via laser desorption mass spectrometry (LD-MS) without a matrix ${ }^{\mathrm{S} 1}$ and by high-resolution fast atom bombardment mass spectrometry (FAB-MS) using a matrix of nitrobenzyl alcohol and polyethylene glycol. Melting points are uncorrected. Silica gel (40 $\mu \mathrm{m}$ average particle size) was used for column chromatography. THF was freshly distilled from sodium as required. Toluene was distilled from $\mathrm{CaH}_{2} . \mathrm{CHCl}_{3}$ was stabilized with $0.8 \%$ ethanol.

Sonication of crude reaction samples was performed (typically to remove chromatographic residue) with use of a benchtop low-power sonication bath. In this process, the crude solid product was suspended in a poor solvent (e.g., a porphyrin in methanol). The suspension was sonicated for a short period ( $<1 \mathrm{~min}$, typically some $5-10 \mathrm{sec})$. The suspension was either decanted (if sonication was to be repeated) or filtered to isolate the solid product.

\section{Noncommercial compounds.}

Aldehydes 15f, ${ }^{31} \mathbf{1 5 g},{ }^{32}$ and $\mathbf{1 5 m} ;{ }^{45}$ dipyrromethanes $\mathbf{1 6 a},{ }^{27} \mathbf{1 6 b},{ }^{13} \mathbf{1 6 c},{ }^{28} \mathbf{1 6 d},{ }^{29} \mathbf{1 6 e},{ }^{23}$ $\mathbf{1 6 h},{ }^{27} \mathbf{1 6} \mathbf{i}^{5}{ }^{\mathbf{1 6}} \mathbf{j}^{30}$ and tri(pyrrol-2-yl)methane (16k); ${ }^{36} 1,9$-diacyldipyrromethane $\mathbf{1 8}^{33}$ and tin complex 21; ${ }^{37}$ and porphyrins $\mathbf{Z n - 2 4},{ }^{1} \mathbf{Z n - 2 5},{ }^{1} \mathbf{Z n - 2 6},{ }^{41} \mathbf{2 9},{ }^{44} \mathbf{3 8},{ }^{35}$ and $\mathbf{4 2}{ }^{30}$ were prepared as described in the literature. The synthesis of porphyrin $\mathbf{Z n - 2 3}{ }^{40}$ is described in the Supporting Information of reference 40.

\section{Experimental Procedures.}

5-[4-(4-Allylhepta-1,6-dien-4-yl)phenyl]-10,20-di-p-tolylporphyrin (3). As described for $\mathbf{2}$, the reduction of $18(106 \mathrm{mg}, 0.280 \mathrm{mmol})$ in THF/MeOH $(12 \mathrm{~mL}, 3: 1)$ with $\mathrm{NaBH}_{4}(500$ $\mathrm{mg}, 13.2 \mathrm{mmol}$ ) for $2 \mathrm{~h}$ afforded 18-diol as a slightly yellow foam-like solid, which was condensed with $\mathbf{1 6 b}(100 \mathrm{mg}, 0.280 \mathrm{mmol})$ in $\mathrm{CH}_{2} \mathrm{Cl}_{2}(112 \mathrm{~mL})$ containing $\mathrm{Yb}(\mathrm{OTf})_{3}(220 \mathrm{mg}$, $3.2 \mathrm{mM}, 0.355 \mathrm{mmol}$ ) at room temperature for $30 \mathrm{~min}$ followed by oxidation with DDQ (190 $\mathrm{mg}, 0.837 \mathrm{mmol})$. Standard workup including filtration through a pad of alumina $\left(\mathrm{CH}_{2} \mathrm{Cl}_{2}\right)$ and sonication of the crude porphyrin product suspended in methanol afforded a purple solid (25 $\mathrm{mg}$, 13\%): ${ }^{1} \mathrm{H}$ NMR $\delta-2.98(\mathrm{~s}, 2 \mathrm{H}), 2.72-2.75$ (overlapping peaks $\left.12 \mathrm{H}\right), 5.84-5.92(\mathrm{~m}, 6 \mathrm{H}), 5.84-$ $9.94(\mathrm{~m}, 3 \mathrm{H}), 7.59$ (d, $J=7.6 \mathrm{~Hz}, 4 \mathrm{H}), 7.68(\mathrm{~d}, J=8.3 \mathrm{~Hz}, 2 \mathrm{H}), 8.14$ (d, $J=7.6 \mathrm{~Hz}, 4 \mathrm{H}), 8.16$ (d, $J=8.5 \mathrm{~Hz}, 2 \mathrm{H}), 8.85(\mathrm{~d}, J=4.6 \mathrm{~Hz}, 2 \mathrm{H}), 8.94(\mathrm{~d}, J=4.6 \mathrm{~Hz}, 2 \mathrm{H}), 9.05(\mathrm{~d}, J=4.6 \mathrm{~Hz}, 2 \mathrm{H}), 9.33$ 
(d, $J=4.6 \mathrm{~Hz}, 2 \mathrm{H}), 10.20$ (s, 1H); LD-MS obsd 700.1; FAB-MS obsd 700.3609, calcd 700.3566 $\left(\mathrm{C}_{50} \mathrm{H}_{44} \mathrm{~N}_{4}\right) ; \lambda_{\text {abs }} 414,509,545,584,639 \mathrm{~nm}$.

5-[4-(4-Allylhepta-1,6-dien-4-yl)phenyl]-15,20-di-p-tolylporphinatozinc(II) (Zn-3) As described for 2, the reduction of $18(53 \mathrm{mg}, 0.14 \mathrm{mmol})$ in THF/MeOH (12 $\mathrm{mL}, 3: 1)$ with $\mathrm{NaBH}_{4}(261 \mathrm{mg}, 6.90 \mathrm{mmol})$ for $1.5 \mathrm{~h}$ afforded 18-diol as a slightly yellow foam-like solid, which was condensed with $\mathbf{1 6 b}(50 \mathrm{mg}, 0.14 \mathrm{mmol})$ in $\mathrm{CH}_{2} \mathrm{Cl}_{2}(56 \mathrm{~mL})$ containing $\mathrm{Yb}(\mathrm{OTf})_{3}$ (110 mg, $3.2 \mathrm{mM}, 0.177 \mathrm{mmol}$ ) at room temperature for $30 \mathrm{~min}$ followed by oxidation with DDQ. After standard workup including filtration through a pad of alumina $\left(\mathrm{CH}_{2} \mathrm{Cl}_{2}\right)$, the crude product in $\mathrm{CHCl}_{3}(20 \mathrm{~mL})$ was treated overnight at room temperature with a solution of $\mathrm{Zn}(\mathrm{OAc})_{2} \cdot 2 \mathrm{H}_{2} \mathrm{O}(200 \mathrm{mg}, 0.911 \mathrm{mmol})$ in methanol $(6 \mathrm{~mL})$. Chromatography [silica, hexanes $\left./ \mathrm{CH}_{2} \mathrm{Cl}_{2}(1: 1)\right]$ afforded a purple powder $(11 \mathrm{mg}, 10 \%):{ }^{1} \mathrm{H}$ NMR $\delta 10.22(\mathrm{~s}, 1 \mathrm{H}), 9.38$ $(\mathrm{d}, J=4.6 \mathrm{~Hz}, 2 \mathrm{H}), 9.12(\mathrm{~d}, J=4.6 \mathrm{~Hz}, 2 \mathrm{H}), 9.03(\mathrm{~d}, J=4.6 \mathrm{~Hz}, 2 \mathrm{H}), 8.95(\mathrm{~d}, J=4.6 \mathrm{~Hz}, 2 \mathrm{H})$, $8.16(\mathrm{~d}, J=7.9 \mathrm{~Hz}, 2 \mathrm{H}), 8.13(\mathrm{~d}, J=7.6 \mathrm{~Hz}, 4 \mathrm{H}), 7.68(\mathrm{~d}, J=7.9 \mathrm{~Hz}, 2 \mathrm{H}), 7.58(\mathrm{~d}, J=7.6 \mathrm{~Hz}$, 4H), 5.92-5.85 (m, 3H), 5.23-5.18 (m, 6H), 2.75-2.73 (overlapping peaks, 12H); LD-MS obsd 762.4; FAB-MS obsd 762.2757, calcd 762.2701 $\left(\mathrm{C}_{50} \mathrm{H}_{42} \mathrm{~F}_{4} \mathrm{Zn}\right) ; \lambda_{\text {abs }} 414,542 \mathrm{~nm}$.

\section{5-[4-(4-Allylhepta-1,6-dien-4-yl)phenyl]-15-(4-pyridyl)-10,20-di-p-}

tolylporphinatozinc(II) (Zn-7). As described for $\mathbf{Z n - 5}$, reduction of 19 (70 $\mathrm{mg}, 0.085 \mathrm{mmol})$ in dry THF/MeOH (15 mL, 10:1) with $\mathrm{NaBH}_{4}(160 \mathrm{mg}, 4.23 \mathrm{mmol})$ for $4 \mathrm{~h}$ afforded 19-diol as a slightly yellow foam-like solid, which was condensed with $\mathbf{1 6 d}(20.0 \mathrm{mg}, 2 \mathrm{mM}, 0.089 \mathrm{mmol})$ in $\mathrm{CH}_{2} \mathrm{Cl}_{2}(44 \mathrm{~mL})$ containing $\mathrm{Yb}(\mathrm{OTf})_{3}(87.3 \mathrm{mg}, 3.2 \mathrm{mM}, 0.141 \mathrm{mmol})$ at room temperature for $20 \mathrm{~min}$ followed by oxidation with DDQ $(75 \mathrm{mg}, 0.33 \mathrm{mmol})$. After standard workup including filtration through a pad of alumina $\left(\mathrm{CH}_{2} \mathrm{Cl}_{2} / \mathrm{MeOH}, 3: 1\right)$, the solid was dissolved in $\mathrm{CHCl}_{3}(20$ $\mathrm{mL})$ and metalated with $\mathrm{Zn}(\mathrm{OAc})_{2} \cdot 2 \mathrm{H}_{2} \mathrm{O}(200 \mathrm{mg}, 0.911 \mathrm{mmol})$ in methanol $(6 \mathrm{~mL})$. Standard workup including chromatography (silica, $\mathrm{CH}_{2} \mathrm{Cl}_{2}$ ) and sonication of the product suspended in methanol afforded a purple solid $(5 \mathrm{mg}, 6 \%)$ : ${ }^{1} \mathrm{H}$ NMR $\left(\mathrm{THF}-d_{8}\right) \delta 2.69(\mathrm{~s}, 6 \mathrm{H}), 2.78(\mathrm{~d}, J=7.0$ $\mathrm{Hz}, 6 \mathrm{H}), 5.14-5.23(\mathrm{~m}, 6 \mathrm{H}), 5.88-5.96(\mathrm{~m}, 3 \mathrm{H}), 7.56(\mathrm{~d}, J=8.2 \mathrm{~Hz}, 4 \mathrm{H}), 7.76(\mathrm{~d}, J=8.1 \mathrm{~Hz}$, $2 \mathrm{H}), 8.07(\mathrm{~d}, J=7.7 \mathrm{~Hz}, 4 \mathrm{H}), 8.10-8.15(\mathrm{~m}, 2 \mathrm{H}), 8.16(\mathrm{~d}, J=7.7 \mathrm{~Hz}, 2 \mathrm{H}), 8.80(\mathrm{~d}, J=4.8 \mathrm{~Hz}$, 2H), 8.83-8.88 (overlapping peaks, 4H), 8.88 (d, $J=4.8 \mathrm{~Hz}, 4 \mathrm{H}$ ); LD-MS obsd 840.7; FAB-MS obsd 840.3102, calcd 840.3045 [(M+H) $\left.{ }^{+} ; \mathrm{M}=\mathrm{C}_{55} \mathrm{H}_{45} \mathrm{~N}_{5} \mathrm{Zn}\right]$; $\lambda_{\text {abs }}$ (THF) 424, 556, $597 \mathrm{~nm}$.

\section{5-[4-(4-Allylhepta-1,6-dien-4-yl)phenyl]-15-(4-cyanophenyl)-10,20-di-p-}

tolylporphyrin (8). As described for 2, reduction of $19(110 \mathrm{mg}, 0.134 \mathrm{mmol})$ in dry THF/MeOH $(20 \mathrm{~mL}, 10: 1)$ with $\mathrm{NaBH}_{4}(253 \mathrm{mg}, 6.69 \mathrm{mmol})$ for $4 \mathrm{~h}$ afforded 19-diol as a slightly yellow foam-like solid, which was condensed with 16e $(33.1 \mathrm{mg}, 0.134 \mathrm{mmol})$ in $\mathrm{CH}_{2} \mathrm{Cl}_{2}(53.6 \mathrm{~mL})$ containing $\mathrm{Yb}(\mathrm{OTf})_{3}(106.4 \mathrm{mg}, 3.2 \mathrm{mM}, 0.172 \mathrm{mmol})$ at room temperature for $20 \mathrm{~min}$ followed by oxidation with DDQ $(91.3 \mathrm{mg}, 0.402 \mathrm{mmol})$. Standard workup including chromatography (silica, $\mathrm{CH}_{2} \mathrm{Cl}_{2}$ ) and addition of methanol to the solution containing the product in $\mathrm{CH}_{2} \mathrm{Cl}_{2}$ afforded a purple precipitate, which was collected to give a purple solid (24.6 mg, 23\%): ${ }^{1} \mathrm{H}$ NMR $\delta-2.79(\mathrm{~s}, 2 \mathrm{H}), 2.71(\mathrm{~s}, 6 \mathrm{H}), 2.73(\mathrm{~d}, J=7.0 \mathrm{~Hz}, 6 \mathrm{H}), 5.17-5.23(\mathrm{~m}, 6 \mathrm{H}), 5.81-$ $5.91(\mathrm{~m}, 3 \mathrm{H}), 7.56(\mathrm{~d}, J=8.1 \mathrm{~Hz}, 4 \mathrm{H}), 7.69(\mathrm{~d}, J=8.1 \mathrm{~Hz}, 2 \mathrm{H}), 8.04-8.12(6 \mathrm{H}), 8.15(\mathrm{~d}, J=8.4$ $\mathrm{Hz}, 2 \mathrm{H}), 8.34(\mathrm{~d}, J=8.1 \mathrm{~Hz}, 2 \mathrm{H}), 8.71(\mathrm{~d}, J=4.8 \mathrm{~Hz}, 2 \mathrm{H}), 8.83(\mathrm{~d}, J=4.4 \mathrm{~Hz}, 2 \mathrm{H}), 8.89$ (d, $J=$ $4.4 \mathrm{~Hz}, 2 \mathrm{H}), 8.91(\mathrm{~d}, J=4.8 \mathrm{~Hz}, 2 \mathrm{H})$; LD-MS obsd 802.2; FAB-MS obsd 802.3939, calcd $802.3910\left[(\mathrm{M}+\mathrm{H})^{+} ; \mathrm{M}=\mathrm{C}_{57} \mathrm{H}_{47} \mathrm{~N}_{5}\right] ; \lambda_{\text {abs }} 420,516,552,592,647 \mathrm{~nm}$.

5-[4-(4-Allylhepta-1,6-dien-4-yl)phenyl]-15-(4-cyanophenyl)-10,20-di-ptolylporphinatozinc(II) (Zn-8). As described for $\mathbf{Z n - 4}$, metalation of 8 (20.0 $\mathrm{mg}, 0.025 \mathrm{mmol})$ in $\mathrm{CHCl}_{3}(20 \mathrm{~mL})$ with methanolic $\mathrm{Zn}(\mathrm{OAc})_{2} \cdot 2 \mathrm{H}_{2} \mathrm{O}(200 \mathrm{mg}, 0.911 \mathrm{mmol})$ followed by the standard workup including chromatography (silica, $\mathrm{CH}_{2} \mathrm{Cl}_{2}$ ) and addition of methanol to the 
solution containing the product in $\mathrm{CH}_{2} \mathrm{Cl}_{2}$ afforded a purple precipitate, which was collected to give a purple solid (19 mg, 88\%): ${ }^{1} \mathrm{H}$ NMR $\delta 2.72(\mathrm{~s}, 6 \mathrm{H}), 2.74(\mathrm{~d}, J=7.3 \mathrm{~Hz}, 6 \mathrm{H}), 5.18-5.23$ $(\mathrm{m}, 6 \mathrm{H}), 5.82-5.94(\mathrm{~m}, 3 \mathrm{H}), 7.56(\mathrm{~d}, J=8.1 \mathrm{~Hz}, 4 \mathrm{H}), 7.68(\mathrm{~d}, J=8.9 \mathrm{~Hz}, 2 \mathrm{H}), 8.06(\mathrm{~d}, J=8.1$ $\mathrm{Hz}, 2 \mathrm{H}), 8.10(\mathrm{~d}, J=7.7 \mathrm{~Hz}, 4 \mathrm{H}), 8.16(\mathrm{~d}, J=8.1 \mathrm{~Hz}, 2 \mathrm{H}), 8.35(\mathrm{~d}, J=8.1 \mathrm{~Hz}, 2 \mathrm{H}), 8.81(\mathrm{~d}, J=$ $4.8 \mathrm{~Hz}, 2 \mathrm{H}), 8.94(\mathrm{~d}, J=4.8 \mathrm{~Hz}, 2 \mathrm{H}), 8.99$ (d, $J=4.8 \mathrm{~Hz}, 2 \mathrm{H}), 9.01$ (d, $J=4.8 \mathrm{~Hz}, 2 \mathrm{H})$; LD-MS obsd 863.1; FAB-MS obsd 863.3010, calcd $863.2966\left(\mathrm{C}_{57} \mathrm{H}_{45} \mathrm{~N}_{5} \mathrm{Zn}\right) ; \lambda_{\text {abs }} 422,548,588 \mathrm{~nm}$.

\section{5-[4-(4-Allylhepta-1,6-dien-4-yl)phenyl]-15-[4-(N-(tert-}

butoxycarbonyl)amino)phenyl]-10,20-di-p-tolylporphyrin (9). As described for 2, reduction of $19(215 \mathrm{mg}, 0.26 \mathrm{mmol})$ in dry THF/MeOH $(20 \mathrm{~mL}, 10: 1)$ with $\mathrm{NaBH}_{4}(493 \mathrm{mg}, 0.130 \mathrm{mmol})$ for $4 \mathrm{~h}$ afforded 19-diol as a slightly yellow foam-like solid, which was condensed with $\mathbf{1 6 f}$ (88 $\mathrm{mg}, 0.26 \mathrm{mmol})$ in $\mathrm{CH}_{2} \mathrm{Cl}_{2}(104 \mathrm{~mL})$ containing $\mathrm{Yb}(\mathrm{OTf})_{3}(206 \mathrm{mg}, 3.2 \mathrm{mM}, 0.33 \mathrm{mmol})$ at room temperature for $20 \mathrm{~min}$ followed by oxidation with DDQ (176 mg, $0.775 \mathrm{mmol})$. Standard workup including chromatography (silica, $\mathrm{CH}_{2} \mathrm{Cl}_{2}$ ) and addition of methanol to the solution containing the product in $\mathrm{CH}_{2} \mathrm{Cl}_{2}$ afforded a purple precipitate, which was collected to give a purple solid (75 mg, 32\%): ${ }^{1} \mathrm{H}$ NMR $\delta-2.77(\mathrm{~s}, 2 \mathrm{H}), 1.65(\mathrm{~s}, 9 \mathrm{H}), 2.71(\mathrm{~s}, 6 \mathrm{H}), 2.60(\mathrm{~d}, J=6.9$ $\mathrm{Hz}, 6 \mathrm{H}), 5.17-5.22(\mathrm{~m}, 6 \mathrm{H}), 5.83-5.91(\mathrm{~m}, 3 \mathrm{H}), 6.84(\mathrm{~s}, 1 \mathrm{H}), 7.55(\mathrm{~d}, J=8.1 \mathrm{~Hz}, 4 \mathrm{H}), 7.68(\mathrm{~d}, J$ $=8.1 \mathrm{~Hz}, 2 \mathrm{H}), 7.75(\mathrm{~d}, J=8.4 \mathrm{~Hz}, 2 \mathrm{H}), 8.10(\mathrm{~d}, J=7.7 \mathrm{~Hz}, 4 \mathrm{H}), 8.17-8.12(\mathrm{~m}, 4 \mathrm{H}), 8.81(\mathrm{~d}, J=$ $4.8 \mathrm{~Hz}, 2 \mathrm{H}), 8.87$ (d, $J=4.8 \mathrm{~Hz}, 6 \mathrm{H})$, LD-MS obsd 892.0; FAB-MS obsd 892.4636, calcd $892.4591\left(\mathrm{C}_{61} \mathrm{H}_{57} \mathrm{~N}_{5} \mathrm{O}_{2}\right) ; \lambda_{\text {abs }} 421,518,553,592,655 \mathrm{~nm}$.

\section{5-[4-(4-Allylhepta-1,6-dien-4-yl)phenyl]-15-[4-( $N$-(tert-}

butoxycarbonyl)amino)phenyl]-10,20-di-p-tolylporphinatozinc(II) (Zn-9). As described for $\mathbf{Z n - 4}$, a solution of $9(22.0 \mathrm{mg}, 0.025 \mathrm{mmol})$ in $\mathrm{CHCl}_{3}(20 \mathrm{~mL})$ was treated with a methanolic solution of $\mathrm{Zn}(\mathrm{OAc})_{2} \cdot 2 \mathrm{H}_{2} \mathrm{O}(300 \mathrm{mg}, 1.36 \mathrm{mmol})$ followed by standard workup including chromatography (silica, $\mathrm{CH}_{2} \mathrm{Cl}_{2}$ ), affording a purple solid $(17.0 \mathrm{mg}, 72 \%)$ : ${ }^{1} \mathrm{H}$ NMR $\delta 1.63(\mathrm{~s}$, $9 \mathrm{H}), 2.71(\mathrm{~s}, 6 \mathrm{H}), 2.60(\mathrm{~d}, J=7.4 \mathrm{~Hz}, 6 \mathrm{H}), 5.17-5.23(\mathrm{~m}, 6 \mathrm{H}), 5.83-5.94(\mathrm{~m}, 3 \mathrm{H}), 6.81(\mathrm{~s}, 1 \mathrm{H})$, $7.55(\mathrm{~d}, J=7.7 \mathrm{~Hz}, 4 \mathrm{H}), 7.68(\mathrm{~d}, J=8.1 \mathrm{~Hz}, 2 \mathrm{H}), 7.75(\mathrm{~d}, J=7.7 \mathrm{~Hz}, 2 \mathrm{H}), 8.10(\mathrm{~d}, J=8.1 \mathrm{~Hz}$, 4H), 8.18-8.11 (m, 4H), 8.92 (d, $J=4.7 \mathrm{~Hz}, 2 \mathrm{H}), 8.98$ (d, $J=4.7 \mathrm{~Hz}, 6 \mathrm{H})$; LD-MS obsd 955.3; FAB-MS obsd 953.3706, calcd $953.3647\left(\mathrm{C}_{61} \mathrm{H}_{55} \mathrm{~N}_{5} \mathrm{O}_{2} \mathrm{Zn}\right) ; \lambda_{\text {abs }} 421,559,587 \mathrm{~nm}$.

\section{5-[4-(4-Allylhepta-1,6-dien-4-yl)phenyl]-15-(4-aminophenyl)-10,20-di-p-}

tolylporphinatozinc(II) (Zn-10). As described for $\mathbf{Z n - 4}$, a solution of $\mathbf{1 0}$ (16 $\mathrm{mg}, 0.02 \mathrm{mmol})$ in $\mathrm{CHCl}_{3}(20 \mathrm{~mL})$ was treated with a methanolic solution of $\mathrm{Zn}(\mathrm{OAc})_{2} \cdot 2 \mathrm{H}_{2} \mathrm{O}(200 \mathrm{mg}, 0.911$ mmol) followed by standard workup including chromatography (silica, $\mathrm{CH}_{2} \mathrm{Cl}_{2}$ ), affording a purple solid (15 mg, 87\%): ${ }^{1} \mathrm{H}$ NMR $\left(\right.$ THF- $\left.d_{8}\right) \delta 2.69(\mathrm{~s}, 6 \mathrm{H}), 2.77(\mathrm{~d}, J=7.3 \mathrm{~Hz}, 6 \mathrm{H}), 4.89$ (s, 2H), 5.15-5.23 (6H), 5.88-5.94 (m, 3H), $7.00(\mathrm{~d}, J=8.4 \mathrm{~Hz}, 2 \mathrm{H}), 7.55(\mathrm{~d}, J=7.7 \mathrm{~Hz}, 4 \mathrm{H}), 7.75$ $(\mathrm{d}, J=8.1 \mathrm{~Hz}, 2 \mathrm{H}), 7.86(\mathrm{~d}, J=8.1 \mathrm{~Hz}, 2 \mathrm{H}), 8.07(\mathrm{~d}, J=7.7 \mathrm{~Hz}, 4 \mathrm{H}), 8.16(\mathrm{~d}, J=8.1 \mathrm{~Hz}, 2 \mathrm{H})$, 8.80-8.94 (m, 6H), 8.95 (d, $J=4.8,2 \mathrm{H})$; LD-MS obsd 853.0; FAB-MS obsd 853.3179, calcd $853.3123\left(\mathrm{C}_{56} \mathrm{H}_{47} \mathrm{~N}_{5} \mathrm{Zn}\right) ; \lambda_{\text {abs }} 422,551,593 \mathrm{~nm}$.

\section{5-[4-(4-Allylhepta-1,6-dien-4-yl)phenyl]-15-(4-amino-3,5-dimethylphenyl)-10,20-di-} p-tolylporphyrin (11). As described for 2, reduction of 21 (100 $\mathrm{mg}, 0.12 \mathrm{mmol})$ in dry THF/MeOH $(20 \mathrm{~mL}, 10: 1)$ with $\mathrm{NaBH}_{4}(300 \mathrm{mg}$, $7.93 \mathrm{mmol})$ for $4 \mathrm{~h}$ afforded 19-diol as a slightly yellow foam-like solid, which was condensed with $\mathbf{1 6 g}(33 \mathrm{mg}, 0.12 \mathrm{mmol})$ in $\mathrm{CH}_{2} \mathrm{Cl}_{2}$ $(48 \mathrm{~mL})$ containing $\mathrm{Yb}(\mathrm{OTf})_{3}(95.3 \mathrm{mg}, 3.2 \mathrm{mM}, 0.154 \mathrm{mmol})$ at room temperature for $40 \mathrm{~min}$ followed by oxidation with $p$-chloranil $(200 \mathrm{mg}, 0.81 \mathrm{mmol})$ for $2 \mathrm{~h}$. Standard workup including chromatography (silica, $\mathrm{CH}_{2} \mathrm{Cl}_{2}$ ) and addition of methanol to the solution containing the product in $\mathrm{CH}_{2} \mathrm{Cl}_{2}$ afforded a purple precipitate, which was collected to give a purple solid (20 mg, 20\%): ${ }^{1} \mathrm{H}$ NMR (300 MHz) $\delta-2.74(\mathrm{~s}, 2 \mathrm{H}), 2.47$ (s, 6H), $2.70(\mathrm{~s}, 6 \mathrm{H}), 2.74(\mathrm{~d}, J=7.3 \mathrm{~Hz}, 6 \mathrm{H}), 3.94$ 
(br, 2H), 5.17-5.23 (m, 6H), 5.80-5.92 (m, 3H), $7.55(\mathrm{~d}, J=7.7 \mathrm{~Hz}, 4 \mathrm{H}), 7.68(\mathrm{~d}, J=8.1 \mathrm{~Hz}$, 2H), 7,81 (s, 2H), $8.10(\mathrm{~d}, J=7.7 \mathrm{~Hz}, 4 \mathrm{H}), 8.16(\mathrm{~d}, J=7.7 \mathrm{~Hz}, 2 \mathrm{H}), 8.80(\mathrm{~d}, J=4.8 \mathrm{~Hz}, 2 \mathrm{H})$, 8.81-8.87 (m, 4H), 8.94 (d, $J=4.4 \mathrm{~Hz}, 2 \mathrm{H})$; LD-MS obsd 820.6; FAB-MS obsd 820.4392 calcd $820.4379\left[(\mathrm{M}+\mathrm{H})^{+} ; \mathrm{M}=\mathrm{C}_{58} \mathrm{H}_{53} \mathrm{~N}_{5}\right] ; \lambda_{\text {abs }} 421,519,557,593,561 \mathrm{~nm}$.

5-[4-(4-Allylhepta-1,6-dien-4-yl)phenyl]-15-(4-amino-3,5-dimethylphenyl)-10,20-dip-tolylporphinatozinc(II) (Zn-11). As described for $\mathbf{Z n - 4}$, treatment of a solution of 11 (10 $\mathrm{mg}, 0.012 \mathrm{mmol})$ in $\mathrm{CHCl}_{3}(20 \mathrm{~mL})$ with methanolic $\mathrm{Zn}(\mathrm{OAc})_{2} \cdot 2 \mathrm{H}_{2} \mathrm{O}(200 \mathrm{mg}, 0.911 \mathrm{mmol})$ followed by standard workup including chromatography (silica, $\mathrm{CH}_{2} \mathrm{Cl}_{2}$ ) afforded a purple solid (10 mg, 93\%): ${ }^{1} \mathrm{H}$ NMR $\delta 2.00(\mathrm{~s}, 6 \mathrm{H}), 2.70(\mathrm{~s}, 6 \mathrm{H}), 2.73$ (d, $\left.J=7.0 \mathrm{~Hz}, 6 \mathrm{H}\right), 3.10$ (br, 2H), $5.16-5.22(\mathrm{~m}, 6 \mathrm{H}), 5.82-5.91(\mathrm{~m}, 3 \mathrm{H}), 7.53(\mathrm{~d}, J=7.7 \mathrm{~Hz}, 4 \mathrm{H}), 7.66(\mathrm{~d}, J=8.8 \mathrm{~Hz}, 4 \mathrm{H}), 8.10$ $(\mathrm{d}, J=7.7 \mathrm{~Hz}, 4 \mathrm{H}), 8.17(\mathrm{~d}, J=8.1 \mathrm{~Hz}, 2 \mathrm{H}), 8.90-8.93(\mathrm{~m}, 4 \mathrm{H}), 8.93-8.98(\mathrm{~m}, 4 \mathrm{H})$ LD-MS obsd 881.5; FAB-MS obsd 881.3474 calcd $881.3436\left(\mathrm{C}_{58} \mathrm{H}_{51} \mathrm{~N}_{5} \mathrm{Zn}\right) ; \lambda_{\text {abs }} 423,551,591 \mathrm{~nm}$.

5,15-Bis[4-(4-allylhepta-1,6-dien-4-yl)phenyl]-10,20-dimesitylporphinatozinc(II)

(Zn-12). As described for $\mathbf{Z n - 2}$, a solution of $12(16.0 \mathrm{mg}, 0.016 \mathrm{mmol})$ in $\mathrm{CHCl}_{3}(20 \mathrm{~mL})$ was treated with a methanolic solution of $\mathrm{Zn}(\mathrm{OAc})_{2} \cdot 2 \mathrm{H}_{2} \mathrm{O}(200 \mathrm{mg}, 0.911 \mathrm{mmol})$ followed by standard workup including chromatography (silica, $\mathrm{CH}_{2} \mathrm{Cl}_{2}$ with $20 \%$ of $\mathrm{CHCl}_{3}$ ) to give a purple solid (16.5 mg, 97\%): ${ }^{1} \mathrm{H}$ NMR $\delta 1.82(\mathrm{~s}, 12 \mathrm{H}), 2.62(\mathrm{~s}, 6 \mathrm{H}), 2.73(\mathrm{~d}, J=7.0 \mathrm{~Hz}, 12 \mathrm{H}), 5.15-$ $5.21(\mathrm{~m}, 12 \mathrm{H}), 5.80-5.90(\mathrm{~m}, 6 \mathrm{H}), 7.26(\mathrm{~s}, 4 \mathrm{H}), 7.66(\mathrm{~d}, J=8.1 \mathrm{~Hz}, 4 \mathrm{H}), 8.17(\mathrm{~d}, J=8.4 \mathrm{~Hz}$, 4H), $8.77(\mathrm{~d}, J=4.8 \mathrm{~Hz}, 4 \mathrm{H}), 8.85(\mathrm{~d}, J=4.8 \mathrm{~Hz}, 4 \mathrm{H})$; LD-MS obsd 1028.2; FAB-MS obsd 1028.4744; calcd $1028.4735\left(\mathrm{C}_{70} \mathrm{H}_{68} \mathrm{~N}_{4} \mathrm{Zn}\right)$; $\lambda_{\text {abs }} 421,549,588 \mathrm{~nm}$.

5-(2-Pyrrolyl)-10,20-di-p-tolyl-15-(4-vinylphenyl)porphyrin (13). As described for 2, reduction of $20(217 \mathrm{mg}, 0.303 \mathrm{mmol})$ in dry THF/MeOH $(14 \mathrm{~mL}, 6: 1)$ with $\mathrm{NaBH}_{4}(460 \mathrm{mg}$, $12.2 \mathrm{mmol}$ ) for $4 \mathrm{~h}$ afforded 20-diol as a slightly yellow foam-like solid, which was condensed with 16k (64.0 mg, $0.303 \mathrm{mmol})$ in $\mathrm{CH}_{2} \mathrm{Cl}_{2}(160 \mathrm{~mL})$ containing $\mathrm{Yb}(\mathrm{OTf})_{3}(317 \mathrm{mg}, 3.2 \mathrm{mM}$, $0.511 \mathrm{mmol}$ ) at room temperature for $20 \mathrm{~min}$ followed by oxidation with DDQ (272 $\mathrm{mg}, 1.20$ mmol). Standard workup including filtration through a pad of alumina $\left(\mathrm{CH}_{2} \mathrm{Cl}_{2}\right)$ and chromatography (silica, $\mathrm{CH}_{2} \mathrm{Cl}_{2}$ ) afforded a purple solid $(20 \mathrm{mg}, 10 \%):{ }^{1} \mathrm{H} \mathrm{NMR} \delta-2.70(\mathrm{~s}, 2 \mathrm{H})$, $2.71(\mathrm{~s}, 6 \mathrm{H}), 5.42(\mathrm{~d}, J=11.2 \mathrm{~Hz}, 1 \mathrm{H}), 6.07(\mathrm{~d}, J=17.6 \mathrm{~Hz}, 1 \mathrm{H}), 6.74-6.77(\mathrm{~m}, 1 \mathrm{H}), 7.06(\mathrm{dd}, J$ $=17.6 \mathrm{~Hz}, J=11.2 \mathrm{~Hz}, 1 \mathrm{H}), 7.20-7.23(\mathrm{~m}, 1 \mathrm{H}), 7.28-7.31(\mathrm{~m}, 1 \mathrm{H}), 7.57(\mathrm{~d}, J=7.8 \mathrm{~Hz}, 4 \mathrm{H})$, $7.79(\mathrm{~d}, J=8.1 \mathrm{~Hz}, 2 \mathrm{H}), 8.10(\mathrm{~d}, J=7.8 \mathrm{~Hz}, 4 \mathrm{H}), 8.17(\mathrm{~d}, J=8.1 \mathrm{~Hz}, 2 \mathrm{H}), 8.86-8.85(\mathrm{~m}, 4 \mathrm{H})$, $8.87(\mathrm{~d}, J=4.8 \mathrm{~Hz}, 2 \mathrm{H}), 9.06(\mathrm{~d}, J=4.6 \mathrm{~Hz}, 2 \mathrm{H}), 9.17(\mathrm{~s}, 1 \mathrm{H})$; LD-MS 657.4; FAB-MS obsd 658.2958, calcd 658.2971 [(M+H) $\left.{ }^{+} ; \mathrm{M}=\mathrm{C}_{46} \mathrm{H}_{35} \mathrm{~N}_{5}\right] ; \lambda_{\text {abs }} 422,519,557,593,551 \mathrm{~nm}$.

5-(2-Pyrrolyl)-10,20-di-p-tolyl-15-(4-vinylphenyl)porphinatozinc(II) (Zn-13). As described for $\mathbf{Z n - 4}$, a solution of $\mathbf{1 3}(7.0 \mathrm{mg}, 0.011 \mathrm{mmol})$ in $\mathrm{CHCl}_{3}(20 \mathrm{~mL})$ was treated with a methanolic solution of $\mathrm{Zn}(\mathrm{OAc})_{2} \cdot 2 \mathrm{H}_{2} \mathrm{O}(200 \mathrm{mg}, 0.911 \mathrm{mmol})$ followed by standard workup including chromatography (silica, $\mathrm{CH}_{2} \mathrm{Cl}_{2}$ ) and addition of methanol to the solution containing the product in $\mathrm{CH}_{2} \mathrm{Cl}_{2}$ afforded a purple solid (4.0 mg, 52\%): ${ }^{1} \mathrm{H}$ NMR $\delta 2.72(\mathrm{~s}, 6 \mathrm{H}), 5.49(\mathrm{~d}, J$ $=11.2 \mathrm{~Hz}, 1 \mathrm{H}), 6.07(\mathrm{~d}, J=17.6 \mathrm{~Hz}, 1 \mathrm{H}), 6.77-6.80(\mathrm{~m}, 1 \mathrm{H}) ; 7.06(\mathrm{dd}, J=17.6 \mathrm{~Hz}, J=11.2 \mathrm{~Hz}$, $1 \mathrm{H}), 7.18-7.21(\mathrm{~m}, 1 \mathrm{H}), 7.36-7.39(\mathrm{~m}, 1 \mathrm{H}), 7.56(\mathrm{~d}, J=8.0 \mathrm{~Hz}, 4 \mathrm{H}), 7.79(\mathrm{~d}, J=8.0 \mathrm{~Hz}, 2 \mathrm{H})$, $8.08(\mathrm{~d}, J=8.0 \mathrm{~Hz}, 4 \mathrm{H}), 8.16(\mathrm{~d}, J=8.0 \mathrm{~Hz}, 2 \mathrm{H}), 8.87-8.95(\mathrm{~m}, 4 \mathrm{H}), 8.98(\mathrm{~d}, J=4.6 \mathrm{~Hz}, 2 \mathrm{H})$, $9.19(\mathrm{~d}, J=4.6 \mathrm{~Hz}, 2 \mathrm{H}), 9.26$ (s, 1H); LD-MS 719.7; FAB-MS obsd 719.2027, calcd 719.2027 $\left(\mathrm{C}_{46} \mathrm{H}_{33} \mathrm{~N}_{5} \mathrm{Zn}\right) ; \lambda_{\text {abs }} 425,552,597 \mathrm{~nm}$.

5-(4-Pyridyl)-10,20-di-p-tolyl-15-(4-vinylphenyl)porphinatozinc(II) (Zn-14). As described for Zn-5 with slight modification, reduction of 20 (143 $\mathrm{mg}, 0.200 \mathrm{mmol})$ in dry THF/MeOH (10 mL, 10:1) with $\mathrm{NaBH}_{4}(152 \mathrm{mg}, 4.02 \mathrm{mmol})$ was incomplete after $2 \mathrm{~h}$ upon 
TLC analysis [hexanes/ethyl acetate (3:1)]. An identical amount of $\mathrm{NaBH}_{4}$ was added, causing complete reduction after $2 \mathrm{~h}$ and giving 20-diol as a slightly yellow foam-like solid. Condensation of 20-diol and $\mathbf{1 6 d}(45 \mathrm{mg}, 0.20 \mathrm{mmol})$ in $\mathrm{CH}_{2} \mathrm{Cl}_{2}(80 \mathrm{~mL})$ containing $\mathrm{Yb}(\mathrm{OTf})_{3}$ $(158 \mathrm{mg}, 0.255 \mathrm{mmol})$ at room temperature for $30 \mathrm{~min}$ followed by oxidation with DDQ (200 $\mathrm{mg}, 0.881 \mathrm{mmol})$ and standard workup including filtration through a pad of alumina $\left(\mathrm{CHCl}_{3}\right)$ afforded the crude free base porphyrin. A solution of the crude product in $\mathrm{CHCl}_{3}(15 \mathrm{~mL})$ was treated with a methanolic solution of $\mathrm{Zn}(\mathrm{OAc})_{2} \cdot 2 \mathrm{H}_{2} \mathrm{O}(200 \mathrm{mg}, 0.911 \mathrm{mmol})$ followed by the standard workup including chromatography (silica, $\mathrm{CH}_{2} \mathrm{Cl}_{2}$ ) and addition of methanol to the solution containing the product in $\mathrm{CH}_{2} \mathrm{Cl}_{2}$ afforded a purple precipitate, which was collected to give a purple powder $(18 \mathrm{mg}, 12 \%)$ : ${ }^{1} \mathrm{H}$ NMR $\left(\mathrm{THF}-d_{8}\right) \delta 2.69(\mathrm{~s}, 6 \mathrm{H}), 5.44(\mathrm{dd}, J=11.0 \mathrm{~Hz}, J=$ $0.9 \mathrm{~Hz}, 1 \mathrm{H}), 6.10(\mathrm{dd}, J=17.5 \mathrm{~Hz}, J=0.9 \mathrm{~Hz}, 1 \mathrm{H}), 7.08(\mathrm{dd}, J=17.5 \mathrm{~Hz}, J=11.0 \mathrm{~Hz}, 1 \mathrm{H}), 7.57$ $(\mathrm{d}, J=7.8 \mathrm{~Hz}, 4 \mathrm{H}), 7.84(\mathrm{~d}, J=7.8 \mathrm{~Hz}, 2 \mathrm{H}), 8.11-8.04(\mathrm{~m}, 6 \mathrm{H}), 8.16(\mathrm{~d}, J=7.8 \mathrm{~Hz}, 2 \mathrm{H}), 8.82-$ $8.76(\mathrm{~m}, 4 \mathrm{H}), 8.91-8.85$ (m, 6H); LD-MS obsd 731.2; FAB-MS obsd 731.2073, calcd 731.2027 $\left(\mathrm{C}_{47} \mathrm{H}_{33} \mathrm{~N}_{5} \mathrm{Zn}\right) ; \lambda_{\text {abs }}$ (THF) $422,550 \mathrm{~nm}$.

\section{Dibutyl[5-(4-(4-allylhepta-1,6-dien-4-yl)phenyl)-5,10-dihydro-1,9-di-p-}

toluoyldipyrrinato]tin(IV) (19). As described for 17, EtMgBr $(6.8 \mathrm{~mL}, 6.8 \mathrm{mmol}, 1.0 \mathrm{M}$ in THF) was added slowly to a tap-water cooled flask containing a solution of $\mathbf{1 6 b}(488 \mathrm{mg}, 1.37$ $\mathrm{mmol})$ in toluene $(20 \mathrm{~mL})$ under argon. The reaction mixture was stirred at room temperature for $30 \mathrm{~min}$. A sample of $p$-toluoyl chloride $(0.37 \mathrm{~mL}, 2.9 \mathrm{mmol})$ was added over $10 \mathrm{~min}$. The mixture was stirred for an additional $25 \mathrm{~min}$ and then the reaction mixture was poured into a mixture of saturated aqueous $\mathrm{NH}_{4} \mathrm{Cl}$ and ethyl acetate. The organic layer was washed (water and brine), dried $\left(\mathrm{Na}_{2} \mathrm{SO}_{4}\right)$, and concentrated to dryness. The residue was treated with TEA $(0.4 \mathrm{~mL})$ and $\mathrm{Bu}_{2} \mathrm{SnCl}_{2}(400 \mathrm{mg}, 1.32 \mathrm{mmol})$ in $\mathrm{CH}_{2} \mathrm{Cl}_{2}(15 \mathrm{~mL})$ at room temperature for $30 \mathrm{~min}$. The mixture was concentrated, chromatographed (silica, $\mathrm{CH}_{2} \mathrm{Cl}_{2}$ with $1 \%$ of TEA), and the solution containing the product in diethyl ether was treated with methanol to give a pale yellow powder (405 mg, 36\%): mp 145-150 (dec.); ${ }^{1} \mathrm{H}$ NMR $\delta 0.68$ (t, $\left.J=7.3 \mathrm{~Hz}, 3 \mathrm{H}\right), 0.72$ (t, $\left.J=7.3 \mathrm{~Hz}, 3 \mathrm{H}\right)$, $1.10-1.43(\mathrm{~m}, 8 \mathrm{H}), 1.50-1.55(\mathrm{~m}, 2 \mathrm{H}), 1.61-1.66(\mathrm{~m}, 2 \mathrm{H}), 2.42(\mathrm{~d}, J=7.3 \mathrm{~Hz}, 6 \mathrm{H}), 2.44(\mathrm{~s}, 6 \mathrm{H})$, 4.97-5.02 (m, 6H), 5.50-5.57 (m, 3H), $5.54(\mathrm{~s}, 1 \mathrm{H}), 6.15(\mathrm{~d}, J=3.8 \mathrm{~Hz}, 2 \mathrm{H}), 7.09$ (d, $J=3.5 \mathrm{~Hz}$, 2H), $7.14(\mathrm{~d}, J=8.3 \mathrm{~Hz}, 2 \mathrm{H}), 7.23(\mathrm{~d}, J=8.3 \mathrm{~Hz}, 2 \mathrm{H}), 7.29(\mathrm{~d}, J=7.9 \mathrm{~Hz}, 4 \mathrm{H}), 7.82(\mathrm{~d}, J=7.9$ $\mathrm{Hz}, 4 \mathrm{H}) ;{ }^{13} \mathrm{C}$ NMR $\delta 13.8,21.8,24.1,24.9,26.2,26.4,27.4,27.5,42.1,43.2,45.3,115.4,117.7$, $123.8,127.10,127.13,128.1,129.27,129.33,134.8,135.2,136.1,144.3,151.8$; FAB-MS obsd 825.3482, calcd $825.3442\left[(\mathrm{M}+\mathrm{H})^{+} ; \mathrm{M}=\mathrm{C}_{49} \mathrm{H}_{56} \mathrm{~N}_{2} \mathrm{O}_{2} \mathrm{Sn}\right]$.

Dibutyl[5,10-dihydro-1,9-di-p-toluoyl-5-(4-vinylphenyl)dipyrrinato]tin(IV) (20). As described for 17, EtMgBr $(10 \mathrm{~mL}, 10 \mathrm{mmol}, 1.0 \mathrm{M}$ in THF) was added slowly to a tap-water cooled flask containing a solution of $\mathbf{1 6} \mathbf{i}(468 \mathrm{mg}, 1.88 \mathrm{mmol})$ in toluene $(40 \mathrm{~mL})$ under argon. The reaction mixture was stirred at room temperature for $30 \mathrm{~min}$. A sample of $p$-toluoyl chloride $(0.7 \mathrm{~mL}, 5 \mathrm{mmol})$ was added over $10 \mathrm{~min}$. The mixture was stirred for an additional $1 \mathrm{~h}$ and then the reaction mixture was poured into a mixture of saturated aqueous $\mathrm{NH}_{4} \mathrm{Cl}$ and ethyl acetate. The organic layer was washed (water and brine), dried $\left(\mathrm{Na}_{2} \mathrm{SO}_{4}\right)$, and concentrated to dryness. The residue was treated with TEA $(0.8 \mathrm{~mL}, 6 \mathrm{mmol})$ and $\mathrm{Bu}_{2} \mathrm{SnCl}_{2}(0.6 \mathrm{~g}, 2 \mathrm{mmol})$ in $\mathrm{CH}_{2} \mathrm{Cl}_{2}$ $(15 \mathrm{~mL})$ at room temperature for $30 \mathrm{~min}$. The mixture was concentrated. The crude mixture was dissolved in $\mathrm{CH}_{2} \mathrm{Cl}_{2}$ and silica (4 g) was added. The mixture was concentrated. Hexanes (60 $\mathrm{mL}$ ) was added and the mixture was sonicated for $10 \mathrm{~min}$ followed by filtration. The filtered material was washed with hexanes $(250 \mathrm{~mL})$ and the eluent was combined with the initial filtrate. The combined filtrate was concentrated. The resulting residue was dissolved in a minimum amount of diethyl ether, to which methanol was added, affording a pale yellow solid (454 $\mathrm{mg}$, 34\%): mp 134-138 ${ }^{\circ} \mathrm{C}$ (dec.); ${ }^{1} \mathrm{H}$ NMR $\delta 0.68$ (t, $\left.J=6.8 \mathrm{~Hz}, 3 \mathrm{H}\right), 0.73$ (t, $\left.J=6.8 \mathrm{~Hz}, 3 \mathrm{H}\right), 1.52-$ 
$1.05(\mathrm{~m}, 10 \mathrm{H}), 1.70-1.63(\mathrm{~m}, 2 \mathrm{H}), 2.43(\mathrm{~s}, 6 \mathrm{H}), 5.20(\mathrm{dd}, J=10.7 \mathrm{~Hz}, J=0.8 \mathrm{~Hz}, 1 \mathrm{H}), 5.58(\mathrm{~s}$, $1 \mathrm{H}), 5.69(\mathrm{dd}, J=17.7 \mathrm{~Hz}, J=0.8 \mathrm{~Hz}, 1 \mathrm{H}), 6.18(\mathrm{~d}, J=3.9 \mathrm{~Hz}, 2 \mathrm{H}), 6.67(\mathrm{dd}, J=17.7 \mathrm{~Hz}, J=$ $10.7 \mathrm{~Hz}, 1 \mathrm{H}), 7.08(\mathrm{~d}, J=3.9 \mathrm{~Hz}, 2 \mathrm{H}), 7.17(\mathrm{~d}, J=8.2 \mathrm{~Hz}, 2 \mathrm{H}), 7.35-7.27(\mathrm{~m}, 6 \mathrm{H}), 7.81(\mathrm{~d}, J=$ 8.2 Hz, 4H); ${ }^{13} \mathrm{C}$ NMR $\delta 13.8,21.8,24.2,25.0,26.2,26.5,27.4,27.5,45.6,113.8,115.3,123.9$, 126.7, 128.5, 129.3, 129.4, 135.2, 136.4, 136.2, 136.7, 142.4, 144.1, 151.4, 184.6; LD-MS obsd 717.8; FAB-MS obsd 717.2540, calcd 717.2503 [(M+H) $\left.{ }^{+} ; \mathrm{M}=\mathrm{C}_{41} \mathrm{H}_{44} \mathrm{~N}_{2} \mathrm{O}_{2} \mathrm{Sn}\right]$; Anal Calcd for $\mathrm{C}_{41} \mathrm{H}_{44} \mathrm{~N}_{2} \mathrm{O}_{2} \mathrm{Sn}: \mathrm{C}, 68.82 ; \mathrm{H}, 6.20 ; \mathrm{N}, 3.92$, Found: C, 68.74; H, 6.15; N, 3.82.

5,10,15-Triphenyl-20-(4-pyridyl)porphinatozinc(II) (Zn-22). As described for Zn-5, reduction of 21 (175 mg, $0.264 \mathrm{mmol})$ in dry THF/MeOH (14 mL, 10:1) with $\mathrm{NaBH}_{4}(400 \mathrm{mg}$, $10.6 \mathrm{mmol}$ ) for $4 \mathrm{~h}$ afforded 21-diol as a slightly yellow foam-like solid, which was condensed with 16d $(59.0 \mathrm{mg}, 1.6 \mathrm{mM}, 0.264 \mathrm{mmol})$ in $\mathrm{CH}_{2} \mathrm{Cl}_{2}(160 \mathrm{~mL})$ containing $\mathrm{Yb}(\mathrm{OTf})_{3}(317 \mathrm{mg}$, $3.2 \mathrm{mM}, 0.511 \mathrm{mmol})$ followed by oxidation with DDQ (272 $\mathrm{mg}, 1.20 \mathrm{mmol})$. Standard workup including filtration through a pad of alumina $\left(\mathrm{CHCl}_{3}\right)$ afforded the crude free base porphyrin, which was dissolved in $\mathrm{CHCl}_{3}(20 \mathrm{~mL})$ and treated with a methanolic solution of $\mathrm{Zn}(\mathrm{OAc})_{2} \cdot 2 \mathrm{H}_{2} \mathrm{O}(250 \mathrm{mg}, 1.14 \mathrm{mmol})$. Standard workup including chromatography (silica, $\mathrm{CH}_{2} \mathrm{Cl}_{2}$ ) and addition of methanol to the solution containing the product in $\mathrm{CH}_{2} \mathrm{Cl}_{2}$ afforded a purple precipitate, which was collected to give a purple powder $(20.0 \mathrm{mg}, 11 \%):{ }^{1} \mathrm{H} \mathrm{NMR}$ (THF$\left.d_{8}\right) \delta$ 7.79-7.71 (m, 9H), 8.08-8.05 (m, 2H), 8.22-8.17 (m, 6H), 8.68-8.64 (m, 2H), $8.76(\mathrm{~d}, J=$ $4.7 \mathrm{~Hz}, 2 \mathrm{H}), 8.85-8.84(\mathrm{~m}, 4 \mathrm{H}), 8.86(\mathrm{~d}, J=4.7 \mathrm{~Hz}, 2 \mathrm{H})$; LD-MS obsd 676.9; FAB-MS 677.1603, calcd $677.1558\left(\mathrm{C}_{43} \mathrm{H}_{27} \mathrm{~N}_{5} \mathrm{Zn}\right) ; \lambda_{\text {abs }}$ (THF) $422,555 \mathrm{~nm}$.

5,15-Bis(4-aminophenyl)-10,20-dimesitylporphinatozinc(II) (Zn-34). As described for Zn-2, a solution of $34(20 \mathrm{mg}, 0.027 \mathrm{mmol})$ in $\mathrm{CHCl}_{3}(20 \mathrm{~mL})$ was treated with methanolic $\mathrm{Zn}(\mathrm{OAc})_{2} \cdot 2 \mathrm{H}_{2} \mathrm{O}(84 \mathrm{mg}, 0.38 \mathrm{mmol})$ followed by standard workup including chromatography ( silica, $\left.\mathrm{CH}_{2} \mathrm{Cl}_{2}\right)$ to give a purple solid $(20 \mathrm{mg}, 92 \%):{ }^{1} \mathrm{H}$ NMR $\left(\mathrm{THF}-d_{8}\right) \delta 1.85(\mathrm{~s}, 12 \mathrm{H}), 2.61(\mathrm{~s}$, $6 \mathrm{H}), 4.87(\mathrm{~s}, 4 \mathrm{H}) ; 6.95(\mathrm{~d}, J=8.1 \mathrm{~Hz}, 4 \mathrm{H}), 7.29(\mathrm{~s}, 4 \mathrm{H}) ; 7.85(\mathrm{~d}, J=8.4 \mathrm{~Hz}, 4 \mathrm{H}), 8.61(\mathrm{~d}, J=4.4$ $\mathrm{Hz}, 4 \mathrm{H}), 8.89$ (d, $J=4.4 \mathrm{~Hz}, 4 \mathrm{H})$; LD-MS obsd 790.9; FAB-MS obsd 790.2732, calcd 790.2762 $\left(\mathrm{C}_{50} \mathrm{H}_{42} \mathrm{~N}_{6} \mathrm{Zn}\right) ; \lambda_{\text {abs }}(\mathrm{THF}) 428,560,556,601 \mathrm{~nm}$.

5,15-Bis(4-isothiocyanatophenyl)-10,20-dimesitylporphinatozinc(II) (Zn-35). As described for $\mathbf{Z n - 2}$, a solution of $\mathbf{3 5}(20 \mathrm{mg}, 0.025 \mathrm{mmol})$ in $\mathrm{CHCl}_{3}(20 \mathrm{~mL})$ was treated with a methanolic solution of $\mathrm{Zn}(\mathrm{OAc})_{2} \cdot 2 \mathrm{H}_{2} \mathrm{O}(60 \mathrm{mg}, 0.27 \mathrm{mmol})$ followed by standard workup including chromatography (silica, $\mathrm{CH}_{2} \mathrm{Cl}_{2}$ ) to give a purple solid $(20 \mathrm{mg}, 93 \%)$ : ${ }^{1} \mathrm{H} \mathrm{NMR} \delta 1.82$ $(\mathrm{s}, 12 \mathrm{H}), 2.64(\mathrm{~s}, 6 \mathrm{H}), 7.29(\mathrm{~s}, 4 \mathrm{H}), 7.62(\mathrm{~d}, J=8.4 \mathrm{~Hz}, 4 \mathrm{H}), 8.22(\mathrm{~d}, J=8.1 \mathrm{~Hz}, 4 \mathrm{H}), 8.79-8.84$ (m, 8H); LD-MS obsd 874.9; FAB-MS obsd 874.1859, calcd 874.1891 $\left(\mathrm{C}_{52} \mathrm{H}_{38} \mathrm{~N}_{6} \mathrm{~S}_{2} \mathrm{Zn}\right) ; \lambda_{\text {abs }}$ (THF) 422, 549, $591 \mathrm{~nm}$.

5,15-(4-Amino-3,5-dimethylphenyl)-10,20-dimesitylporphyrin (36). As described for 12, samples of 16h (208 mg, $10 \mathrm{mM}, 0.844 \mathrm{mmol})$ and aldehyde $\mathbf{1 5 g}(126 \mathrm{mg}, 10 \mathrm{mM}, 0.844$ mmol) were dissolved in $\mathrm{CH}_{2} \mathrm{Cl}_{2}(84.4 \mathrm{~mL})$. TFA $(116 \mu \mathrm{L}, 17.8 \mathrm{mM}, 1.51 \mathrm{mmol})$ was added. The reaction mixture was stirred at room temperature for $30 \mathrm{~min}$. Then $p$-chloranil (309 $\mathrm{mg}, 15$ $\mathrm{mM}, 1.26 \mathrm{mmol}$ ) was added, and the reaction mixture was stirred at room temperature for $2 \mathrm{~h}$. The reaction mixture was neutralized with TEA. The reaction mixture was concentrated and chromatographed (silica, $\mathrm{CH}_{2} \mathrm{Cl}_{2}$ ) to give a purple solid $(16.0 \mathrm{mg}, 5 \%)$ : ${ }^{1} \mathrm{H} \mathrm{NMR} \delta-2.59(\mathrm{~s}, 2 \mathrm{H})$, $1.83(\mathrm{~s}, 12 \mathrm{H}), 2.45(\mathrm{~s}, 12 \mathrm{H}), 2.63(\mathrm{~s}, 6 \mathrm{H}), 3.92(\mathrm{~s}, 4 \mathrm{H}), 7.27(\mathrm{~s}, 4 \mathrm{H}), 7.79(\mathrm{~s}, 4 \mathrm{H}), 8.64(\mathrm{~d}, J=4.8$ $\mathrm{Hz}, 4 \mathrm{H}), 8.87$ (d, $J=4.4 \mathrm{~Hz}, 4 \mathrm{H})$; LD-MS obsd 784.4; FAB-MS obsd 784.4315, calcd 784.4253 $\left(\mathrm{C}_{54} \mathrm{H}_{52} \mathrm{~N}_{6}\right) ; \lambda_{\text {abs }} 424,520,558,595,656 \mathrm{~nm}$. 
5,15-(4-Amino-3,5-dimethylphenyl)-10,20-dimesitylporphinatozinc(II) (Zn-36). As described for $\mathbf{Z n - 2}$, a solution of $\mathbf{3 6}(10.0 \mathrm{mg}, 0.013 \mathrm{mmol})$ in $\mathrm{CHCl}_{3}(20 \mathrm{~mL})$ was treated with a methanolic solution of $\mathrm{Zn}(\mathrm{OAc})_{2} \cdot 2 \mathrm{H}_{2} \mathrm{O}(100 \mathrm{mg}, 0.456 \mathrm{mmol})$ followed by standard workup including chromatography (silica, $\mathrm{CH}_{2} \mathrm{Cl}_{2}$ ) to give a purple solid $(10.0 \mathrm{mg}, 93 \%)$ : ${ }^{1} \mathrm{H} \mathrm{NMR}$ $\left(\mathrm{THF}-d_{8}\right) \delta 1.84(\mathrm{~s}, 12 \mathrm{H}), 2.40(\mathrm{~s}, 12 \mathrm{H}), 2.60(\mathrm{~s}, 6 \mathrm{H}), 4.53(\mathrm{~s}, 4 \mathrm{H}), 7.27(\mathrm{~s}, 4 \mathrm{H}), 7.68(\mathrm{~s}, 4 \mathrm{H}), 8.57$ $(\mathrm{d}, J=4.4 \mathrm{~Hz}, 4 \mathrm{H}), 8.85$ (d, $J=4.8 \mathrm{~Hz}, 4 \mathrm{H})$ LD-MS obsd 846.2; FAB-MS obsd 846.3404, calcd $846.3404\left(\mathrm{C}_{54} \mathrm{H}_{50} \mathrm{~N}_{6} \mathrm{Zn}\right) ; \lambda_{\text {abs }}(\mathrm{THF}) 428,560,601 \mathrm{~nm}$.

5,15-Dimesityl-10,20-bis[(4-methoxycarbonyl)phenyl]porphinatozinc(II) (Zn-39). As described for $\mathbf{1 2}$ with subsequent metalation, condensation of $\mathbf{1 6 h}(120 \mathrm{mg}, 10 \mathrm{mM}, 0.454$ $\mathrm{mmol})$ and $15 \mathrm{l}(74.5 \mathrm{mg}, 10 \mathrm{mM}, 0.454 \mathrm{mmol})$ in $\mathrm{CH}_{2} \mathrm{Cl}_{2}(45.5 \mathrm{~mL})$ containing TFA (62 $\mu \mathrm{L}, 18$ $\mathrm{mM}, 0.81 \mathrm{mmol}$ ) for $30 \mathrm{~min}$ followed by oxidation with DDQ (155 mg, $15 \mathrm{mM}, 0.683 \mathrm{mmol})$ followed by standard workup including silica pad filtration $\left(\mathrm{CH}_{2} \mathrm{Cl}_{2}\right)$ afforded the free base porphyrin. The solid was dissolved in $\mathrm{CHCl}_{3}(20 \mathrm{~mL})$ and treated with methanolic $\mathrm{Zn}(\mathrm{OAc})_{2} \cdot 2 \mathrm{H}_{2} \mathrm{O}(300 \mathrm{mg}, 1.37 \mathrm{mmol})$ followed by standard workup including chromatography ( silica, $\mathrm{CH}_{2} \mathrm{Cl}_{2}$ ) to give a purple solid $(41.0 \mathrm{mg}, 21 \%)$ : ${ }^{1} \mathrm{H}$ NMR $\delta 1.83(\mathrm{~s}, 12 \mathrm{H}), 2.63(\mathrm{~s}, 6 \mathrm{H})$, 4.07 (s, 6H), 7.29 (s, 4H), $8.32(\mathrm{~d}, J=8.1 \mathrm{~Hz}, 4 \mathrm{H}), 8.40(\mathrm{~d}, J=8.1 \mathrm{~Hz}, 4 \mathrm{H}), 8.79-8.85$ (m, 8H); LD-MS obsd 875.8; FAB-MS obsd 876.2629, calcd 876.2654 $\left(\mathrm{C}_{54} \mathrm{H}_{44} \mathrm{~N}_{4} \mathrm{O}_{4} \mathrm{Zn}\right) ; \lambda_{\text {abs }} 421,549$, $589 \mathrm{~nm}$.

5,15-Bis[4-(bromomethyl)phenyl]-10,20-dimesitylporphinatozinc(II) (Zn-40). As described for 12 with subsequent metalation, samples of $\mathbf{1 6 h}(150 \mathrm{mg}, 10 \mathrm{mM}, 0.568 \mathrm{mmol})$ and $15 \mathrm{~m}(113 \mathrm{mg}, 10 \mathrm{mM}, 0.568 \mathrm{mmol})$ were dissolved in $\mathrm{CH}_{2} \mathrm{Cl}_{2}(56.8 \mathrm{~mL})$, and then TFA $(80 \mu \mathrm{L}$, $18 \mathrm{mM}, 1.0 \mathrm{mmol}$ ) was added. The reaction was stirred at room temperature for $30 \mathrm{~min}$. Then DDQ (193 mg, $15 \mathrm{mM}, 0.852 \mathrm{mmol}$ ) was added, and the reaction mixture was stirred for $2 \mathrm{~h}$. The reaction mixture was neutralized with TEA, then poured onto a pad of silica and eluted with $\mathrm{CH}_{2} \mathrm{Cl}_{2}$. The solvent was removed under vacuum. The solid was dissolved in $\mathrm{CHCl}_{3}(20 \mathrm{~mL})$ and treated with a solution of $\mathrm{Zn}(\mathrm{OAc})_{2} \cdot 2 \mathrm{H}_{2} \mathrm{O}(150 \mathrm{mg}, 0.683 \mathrm{mmol})$ in methanol $(10 \mathrm{~mL})$. The mixture was stirred overnight at room temperature. Chromatography (silica, $\mathrm{CH}_{2} \mathrm{Cl}_{2}$ ) afforded a purple solid (58.0 mg; 10\%): ${ }^{1} \mathrm{H}$ NMR $\delta 1.83(\mathrm{~s}, 12 \mathrm{H}), 2.63(\mathrm{~s}, 6 \mathrm{H}), 4.86(\mathrm{~s}, 4 \mathrm{H}), 7.27(\mathrm{~s}, 4 \mathrm{H})$, $7.77(\mathrm{~d}, J=7.7 \mathrm{~Hz}, 4 \mathrm{H}), 8.21(\mathrm{~d}, J=7.7 \mathrm{~Hz}, 4 \mathrm{H}), 8.78(\mathrm{~d}, J=4.8 \mathrm{~Hz}, 4 \mathrm{H}), 8.88$ (d, $J=4.8 \mathrm{~Hz}$, 4H); MALDI-MS (dithranol) obsd 948.2; FAB-MS obsd 944.1110, calcd 944.1068 $\left(\mathrm{C}_{52} \mathrm{H}_{42} \mathrm{Br}_{2} \mathrm{~N}_{4} \mathrm{Zn}\right) ; \lambda_{\text {abs }} 422,549,588 \mathrm{~nm}$.

5,15-Bis[4-(tert-butoxycarbonylamino)phenyl]-10,20-dimesitylporphyrin (41). As described for 12, samples of $\mathbf{1 6 h}(50 \mathrm{mg}, 0.19 \mathrm{mmol})$ and $15 \mathbf{f}(42 \mathrm{mg}, 0.19 \mathrm{mmol})$ and were reacted in $\mathrm{CH}_{2} \mathrm{Cl}_{2}(19 \mathrm{~mL})$ containing TFA $(26 \mu \mathrm{L}, 18 \mathrm{mM}, 0.34 \mathrm{mmol})$. After $30 \mathrm{~min}$, DDQ (65 mg, $15 \mathrm{mM}, 0.29 \mathrm{mmol}$ ) was added, and the reaction mixture was stirred at room temperature for $1 \mathrm{~h}$. The reaction mixture was neutralized by TEA and filtered through a pad of silica $\left(\mathrm{CH}_{2} \mathrm{Cl}_{2}\right)$. The solvent was removed under reduced pressure. Chromatography (silica, $\mathrm{CH}_{2} \mathrm{Cl}_{2}$ ) afforded a purple solid $(35.0 \mathrm{mg}, 40 \%)$ : ${ }^{1} \mathrm{H}$ NMR $\delta-2.62(\mathrm{~s}, 2 \mathrm{H}), 1.65(\mathrm{~s}, 18 \mathrm{H}), 1.84(\mathrm{~s}$, $12 \mathrm{H}), 2.63(\mathrm{~s}, 6 \mathrm{H}), 6.84(\mathrm{~s}, 2 \mathrm{H}), 7.28(\mathrm{~s}, 4 \mathrm{H}), 7.75(\mathrm{~d}, J=7.3 \mathrm{~Hz}, 4 \mathrm{H}), 8.14(\mathrm{~d}, J=8.1 \mathrm{~Hz}, 4 \mathrm{H})$, $8.68(\mathrm{~d}, J=4.8 \mathrm{~Hz}, 4 \mathrm{H}), 8.82$ (d, $J=4.4 \mathrm{~Hz}, 4 \mathrm{H})$; MALDI-MS (dithranol) obsd 929.8; FAB-MS obsd 929.4839, calcd 929.4754 [(M+H) $\left.{ }^{+} ; \mathrm{M}=\mathrm{C}_{60} \mathrm{H}_{60} \mathrm{~N}_{6} \mathrm{O}_{4}\right] ; \lambda_{\text {abs }} 421,446,517,552,592,651$ nm. 


\section{Alternative Synthesis of Porphyrin 42.}

The reaction ${ }^{\mathrm{S} 2}$ of $\mathbf{1 6} \mathbf{j}$ with EtMgBr at $-78^{\circ} \mathrm{C}$ in THF followed by addition of $S$-2-pyridyl thioate $(\mathbf{S 1})^{\mathrm{S3}}$ gave 1-acyldipyrromethane. To facilitate purification of the 1acyldipyrromethane, a boron-complexation strategy ${ }^{\mathrm{S} 4}$ was employed wherein the crude acylation mixture is treated with a dialkylboron triflate and TEA. The resulting 1-acyldipyrromethaneboron complex $\mathbf{S 2}$ was employed as a substrate for 9-acylation. ${ }^{\mathrm{S5}}$ Thus, the reaction of $\mathbf{S 2}$ with $\mathrm{Mes} \mathrm{MgBr}$ followed by the addition of $p$-toluoyl chloride gave the corresponding 1,9diacyldipyrromethane-boron complex $\mathbf{S 3}$ in $52 \%$ yield. Reduction by $\mathrm{NaBH}_{4}$ of the latter afforded the corresponding dipyrromethane-dicarbinol, which upon reaction with dipyrromethane 16j in $\mathrm{CH}_{2} \mathrm{Cl}_{2}$ containing $\mathrm{Yb}(\mathrm{OTf})_{3}$ followed by oxidation with DDQ gave porphyrin $\mathbf{4 2}$ in $14 \%$ yield.

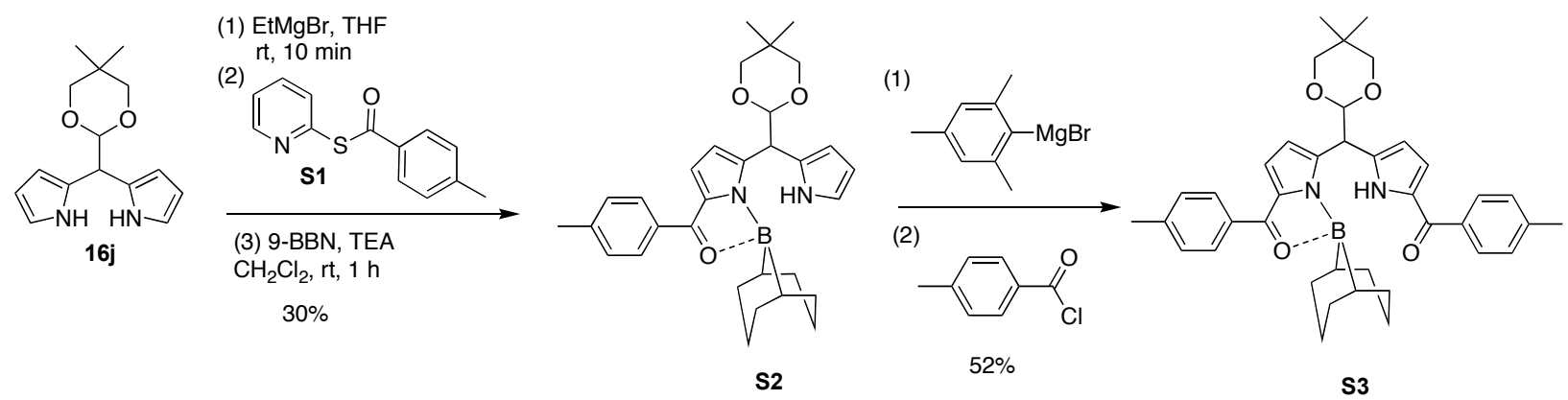

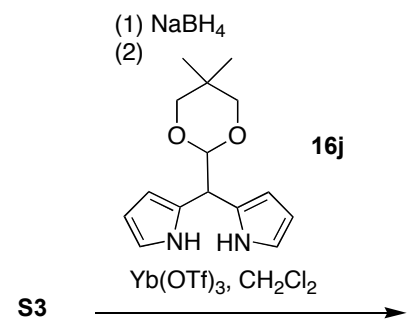

(3) DDQ

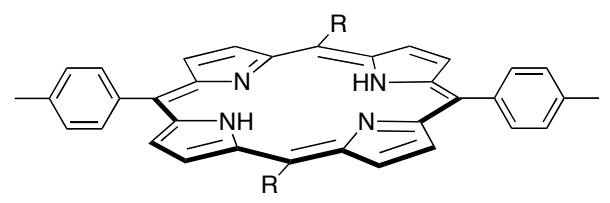

$14 \%$

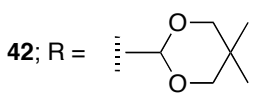

\section{Scheme S1}

10-(9-Borabicyclo[3.3.1]non-9-yl)-1-(4-methylbenzoyl)-5-(5,5-dimethyl-1,3-dioxan-2yl)dipyrromethane (S2). Following a standard acylation-complexation procedure, ${ }^{\mathrm{S} 4}$ a solution of EtMgBr (20.0 mL, $20 \mathrm{mmol}, 1.0 \mathrm{M}$ in THF) was added slowly to a solution of 16j $(2.21 \mathrm{~g}$, $10.0 \mathrm{mmol})$ in THF $(10 \mathrm{~mL})$ under argon. The mixture was stirred at room temperature for 10 min and then cooled to $-78{ }^{\circ} \mathrm{C}$. A solution of $\mathbf{S 1}(2.29 \mathrm{~g}, 10.0 \mathrm{mmol})$ in THF $(10 \mathrm{~mL})$ was added. The solution was stirred at $-78{ }^{\circ} \mathrm{C}$ for $10 \mathrm{~min}$ and then warmed to room temperature. After standard workup, the crude product (a red-orange oil) thus obtained was dissolved in $\mathrm{CH}_{2} \mathrm{Cl}_{2}(20 \mathrm{~mL})$ and treated with TEA $(3.35 \mathrm{~mL}, 24.0 \mathrm{mmol})$ followed by 9-BBN-OTf $(40.0 \mathrm{~mL}$, $20 \mathrm{mmol}, 0.5 \mathrm{M}$ in hexanes) with stirring at room temperature. After $1 \mathrm{~h}$, the mixture was passed through a pad of silica [hexanes $/ \mathrm{CH}_{2} \mathrm{Cl}_{2}(1: 1)$ ], affording a yellow solid (1.5 g, 30\%): $\mathrm{mp} 62-64$ ${ }^{\circ} \mathrm{C} ;{ }^{1} \mathrm{H}$ NMR $\delta$ 0.55-0.65 (m, 1H), 0.67-0.80 (m, 4H), $1.18(\mathrm{~s}, 3 \mathrm{H}), 1.60-2.45(\mathrm{~m}, 12 \mathrm{H}), 2.46(\mathrm{~s}$, $3 \mathrm{H}), 3.44(\mathrm{dd}, J=21.9,11.1 \mathrm{~Hz}, 2 \mathrm{H}), 3.64(\mathrm{dd}, J=11.1, J=2.5 \mathrm{~Hz}, 1 \mathrm{H}), 3.72$ (dd, $J=11.1, J=$ $2.5 \mathrm{~Hz}, 1 \mathrm{H}), 4.82(\mathrm{~d}, J=5.0 \mathrm{~Hz}, 1 \mathrm{H}), 4.93(\mathrm{~d}, J=5.0 \mathrm{~Hz}, 1 \mathrm{H}), 5.65-5.75(\mathrm{~m}, 1 \mathrm{H}), 6.05-6.08$ (m, 
$1 \mathrm{H}), 6.69(\mathrm{~d}, J=4.1 \mathrm{~Hz}, 1 \mathrm{H}), 6.73(\mathrm{~d}, J=1.5 \mathrm{~Hz}, 1 \mathrm{H}), 7.30-7.40(\mathrm{~m}, 3 \mathrm{H}), 8.11(\mathrm{~d}, J=8.2 \mathrm{~Hz}$, 2H), $8.99(\mathrm{~s}, 1 \mathrm{H}) ;{ }^{13} \mathrm{C}$ NMR $\delta 21.9,22.1,23.2,23.9,25.2,26.1,30.1,30.5,34.7,34.9,44.2$, 77.48, 77.54, 77.7, 103.7, 107.8, 108.1, 117.4, 118.0, 120.5, 128.4, 129.3, 129.8, 129.9, 134.6, 144.8, 148.7, 174.0. Anal. Calcd for $\mathrm{C}_{31} \mathrm{H}_{39} \mathrm{BN}_{2} \mathrm{O}_{3}$ : C, 74.70; H, 7.89; N, 5.62. Found: C, 74.69; $\mathrm{H}, 8.13 ; \mathrm{N}, 5.43 . \lambda_{\text {abs }} 379 \mathrm{~nm}$.

\section{0-(9-Borabicyclo[3.3.1]non-9-yl)-5-(5,5-dimethyl-1,3-dioxan-2-yl)-1,9-di-p-toluoyl-}

dipyrromethane (S3). Following a standard procedure, ${ }^{\mathrm{S} 5}$ a suspension of S2 (2.49 g, 5.00 $\mathrm{mmol})$ in THF $(5 \mathrm{~mL})$ under argon was treated with $\mathrm{MesMgBr}(10.0 \mathrm{~mL}, 10 \mathrm{mmol}, 1.0 \mathrm{M}$ in THF). The mixture was stirred at room temperature for $5 \mathrm{~min}$. Then a solution of $p$-toluoyl chloride $(0.727 \mathrm{~mL}, 5.50 \mathrm{mmol})$ in THF $(5.5 \mathrm{~mL})$ was added. The mixture was stirred at room temperature for $10 \mathrm{~min}$ under argon. Standard workup and column chromatography [silica, hexanes $/ \mathrm{CH}_{2} \mathrm{Cl}_{2}(1: 1)$ ] afforded a yellow solid (1.6 g, 52\%): mp $162-164{ }^{\circ} \mathrm{C} ;{ }^{1} \mathrm{H}$ NMR $\delta 0.60-$ $0.66(\mathrm{~m}, 2 \mathrm{H}), 0.74(\mathrm{~s}, 3 \mathrm{H}), 1.23(\mathrm{~s}, 3 \mathrm{H}), 1.65-2.32(\mathrm{~m}, 12 \mathrm{H}), 2.41(\mathrm{~s}, 3 \mathrm{H}), 2.48(\mathrm{~s}, 3 \mathrm{H}), 3.42-$ $3.56(\mathrm{~m}, 2 \mathrm{H}), 3.73(\mathrm{dd}, J=11.0, J=2.5 \mathrm{~Hz}, 1 \mathrm{H}), 3.79(\mathrm{dd}, J=11.0, J=2.5 \mathrm{~Hz}, 1 \mathrm{H}), 4.87(\mathrm{~d}, J=$ $4.1 \mathrm{~Hz}, 1 \mathrm{H}), 5.00(\mathrm{~d}, J=3.8 \mathrm{~Hz}, 1 \mathrm{H}), 5.82-5.88(\mathrm{~m}, 1 \mathrm{H}), 6.68-6.75(\mathrm{~m}, 2 \mathrm{H}), 7.25-7.26(\mathrm{~m}, 2 \mathrm{H})$, $7.35-7.40(\mathrm{~m}, 3 \mathrm{H}), 7.79(\mathrm{~d}, J=8.1 \mathrm{~Hz}, 2 \mathrm{H}), 8.13(\mathrm{~d}, J=8.1 \mathrm{~Hz}, 2 \mathrm{H}), 10.13-10.20(\mathrm{br}, 1 \mathrm{H}) ;{ }^{13} \mathrm{C}$ NMR $\delta$ 21.8, 22.0, 22.1, 23.3, 23.7, 25.1, 26.1, 30.41, 30.45, 30.5, 34.6, 34.8, 44.2, 77.6, 77.9, $103.0,111.3,118.1,119.1,120.6,128.2$, 129.0, 129.1, 129.9, 130.0, 131.0, 134.9, 136.1, 137.2, 142.2, 145.2, 146.9, 174.7, 184.3. Anal. Calcd for $\mathrm{C}_{39} \mathrm{H}_{45} \mathrm{BN}_{2} \mathrm{O}_{4}$ : C, 75.97; H, 7.36; N, 4.54. Found: C, 75.89; H, 7.46; N, 4.35. $\lambda_{\text {abs }} 379 \mathrm{~nm}$.

5,15-Bis(5,5-dimethyl-1,3-dioxan-2-yl)-10,20-di-p-tolylporphyrin (42). Following a standard procedure, ${ }^{23,24}$ a solution of boron complex S3 (166.0 $\left.\mathrm{mg}, 0.269 \mathrm{mmol}\right)$ in dry THF/MeOH (22 mL, 20:2) was treated with $\mathrm{NaBH}_{4}$ (508 mg, $13.5 \mathrm{mmol}, 50$ equiv) in small portions with rapid stirring at room temperature. After $1 \mathrm{~h}$, the reaction was quenched by slow addition of saturated aqueous $\mathrm{NH}_{4} \mathrm{Cl}$. The reaction mixture was extracted with $\mathrm{CH}_{2} \mathrm{Cl}_{2}$. The organic layer was dried and concentrated, affording S3-diol as a slightly yellow foam-like solid. The freshly prepared S3-diol was condensed with $\mathbf{1 6 j}(59.5 \mathrm{mg}, 0.269 \mathrm{mmol})$ in $\mathrm{CH}_{2} \mathrm{Cl}_{2}(107.6$ $\mathrm{mL})$ containing $\mathrm{Yb}(\mathrm{OTf})_{3}(214 \mathrm{mg}, 3.2 \mathrm{mM}, 0.344 \mathrm{mmol})$ at room temperature for $20 \mathrm{~min}$. DDQ (184 mg, $0.811 \mathrm{mmol}$ ) was added, and the reaction mixture was stirred for $1 \mathrm{~h}$. TEA was added to neutralize the acid. The reaction mixture was filtered through a pad of silica $\left(\mathrm{CH}_{2} \mathrm{Cl}_{2}\right)$, affording a purple solid $(27 \mathrm{mg}, 14 \%)$ : ${ }^{1} \mathrm{H}$ NMR $\delta-2.96(\mathrm{~s}, 2 \mathrm{H}), 1.11(\mathrm{~s}, 6 \mathrm{H}), 1.91(\mathrm{~s}, 6 \mathrm{H}), 2.73$ $(\mathrm{s}, 6 \mathrm{H}), 4.27-4.34(\mathrm{~m}, 8 \mathrm{H}), 7.56(\mathrm{~d}, J=7.7 \mathrm{~Hz}, 4 \mathrm{H}), 7.91(\mathrm{~s}, 2 \mathrm{H}), 8.06(\mathrm{~d}, J=8.1 \mathrm{~Hz}, 4 \mathrm{H}), 8.93$ $(\mathrm{d}, J=5.1 \mathrm{~Hz}, 4 \mathrm{H}), 9.89(\mathrm{~d}, J=4.8 \mathrm{~Hz}, 4 \mathrm{H})$; LD-MS obsd 719.0; FAB-MS obsd 719.3594, calcd $719.3597\left[(\mathrm{M}+\mathrm{H})^{+} ; \mathrm{M}=\mathrm{C}_{46} \mathrm{H}_{46} \mathrm{~N}_{4} \mathrm{O}_{4}\right] ; \lambda_{\text {abs }} 415,511,545,588,641 \mathrm{~nm}$.

\section{References}

(S1) (a) Fenyo, D.; Chait, B. T.; Johnson, T. E.; Lindsey, J. S. J. Porphyrins Phthalocyanines 1997, 1, 93-99. (b) Srinivasan, N.; Haney, C. A.; Lindsey, J. S.; Zhang, W.; Chait, B. T. J. Porphyrins Phthalocyanines 1999, 3, 283-291.

(S2) Rao, P. D.; Littler, B. J.; Geier, G. R., III; Lindsey, J. S. J. Org. Chem. 2000, 65, 10841092.

(S3) Araki, M.; Sakata, S.; Takei, H.; Mukaiyama, T. Bull. Chem. Soc. Jpn. 1974, 47, 17771780.

(S4) Muthukumaran, K.; Ptaszek, M.; Noll, B.; Scheidt, W. R.; Lindsey, J. S. J. Org. Chem. 2004, 69, 5354-5364.

(S5) Zaidi, S. H. H.; Muthukumaran, K.; Tamaru, S.-I.; Lindsey, J. S. J. Org. Chem. 2004, $69,8356-8365$. 


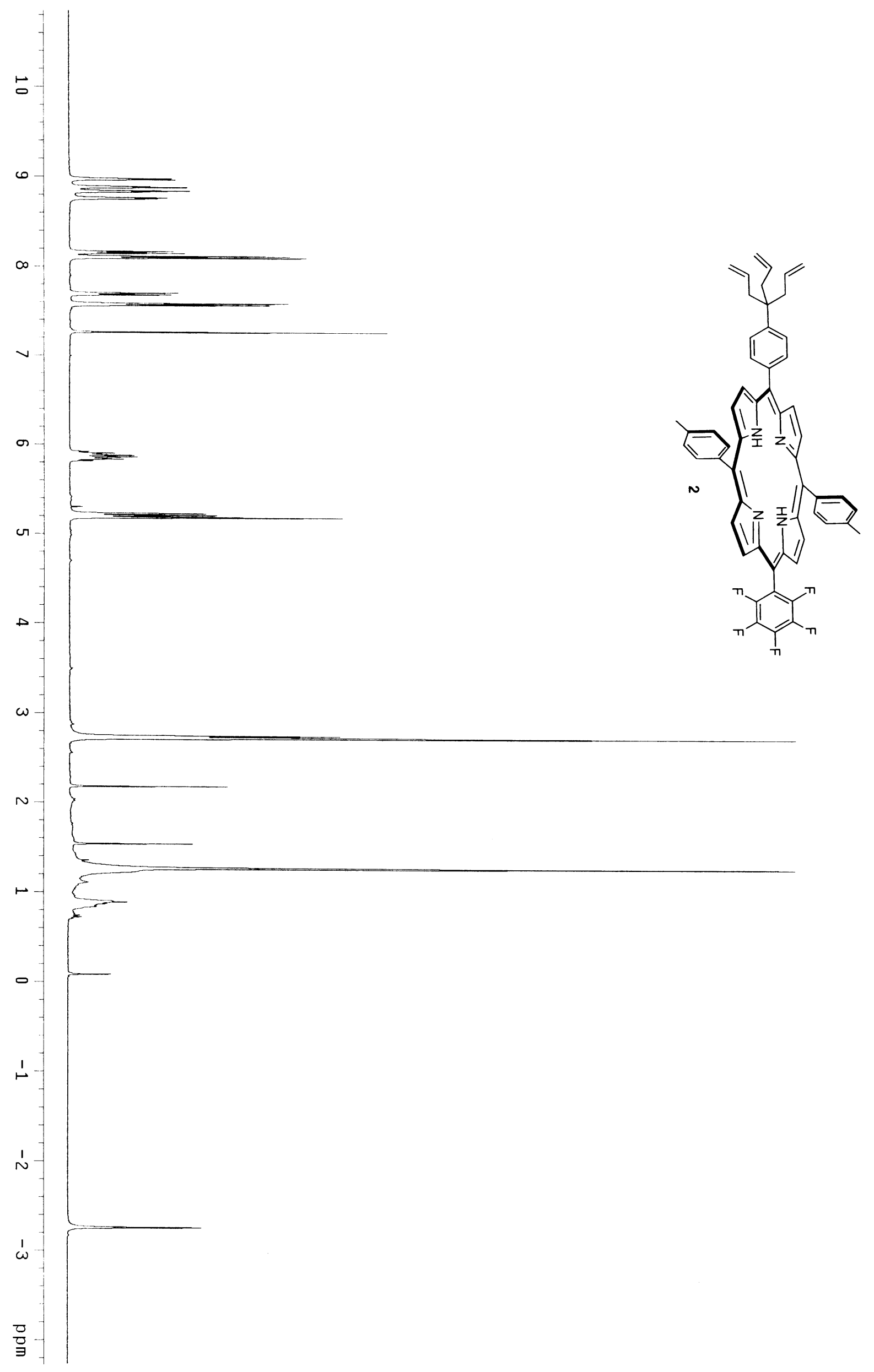




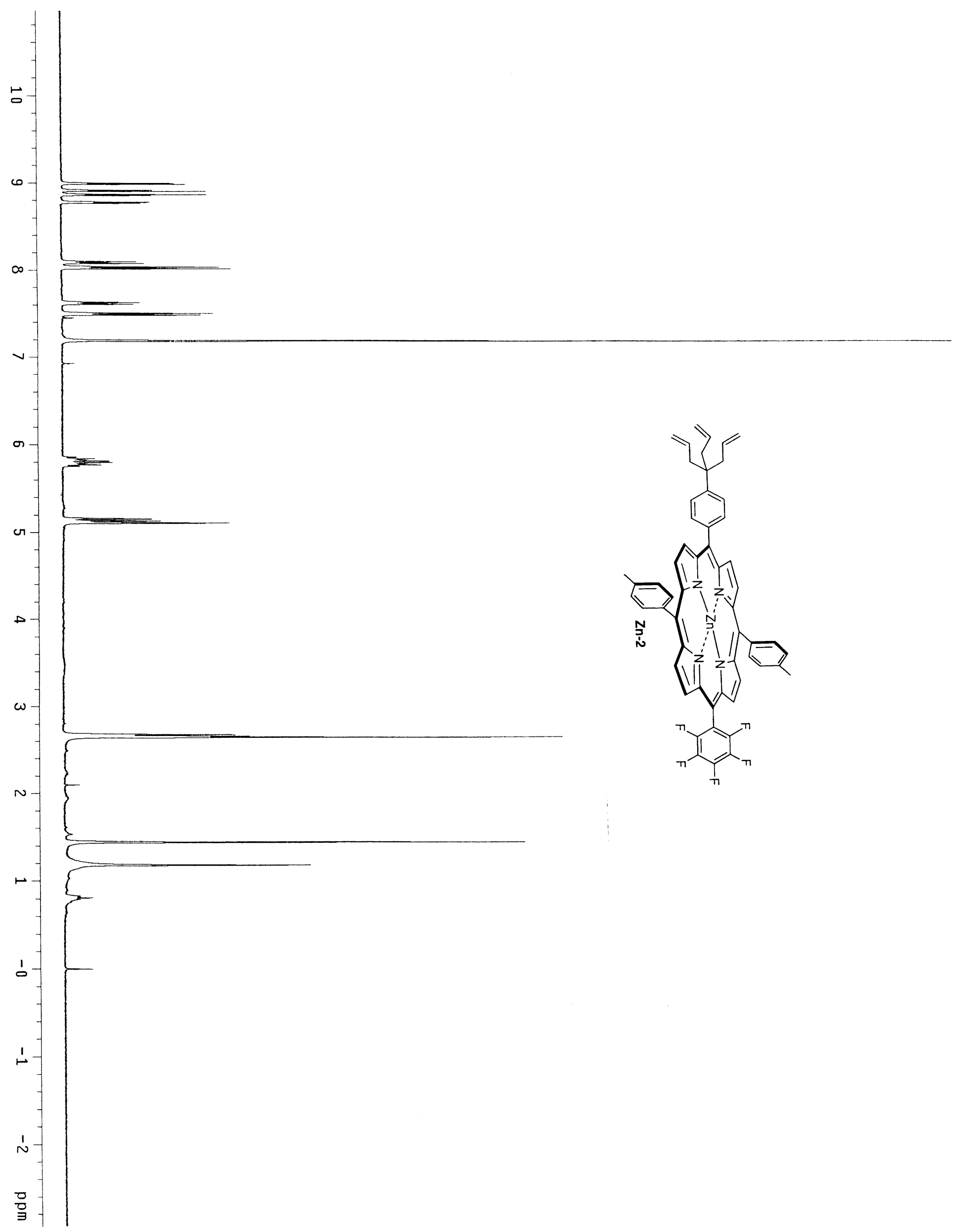




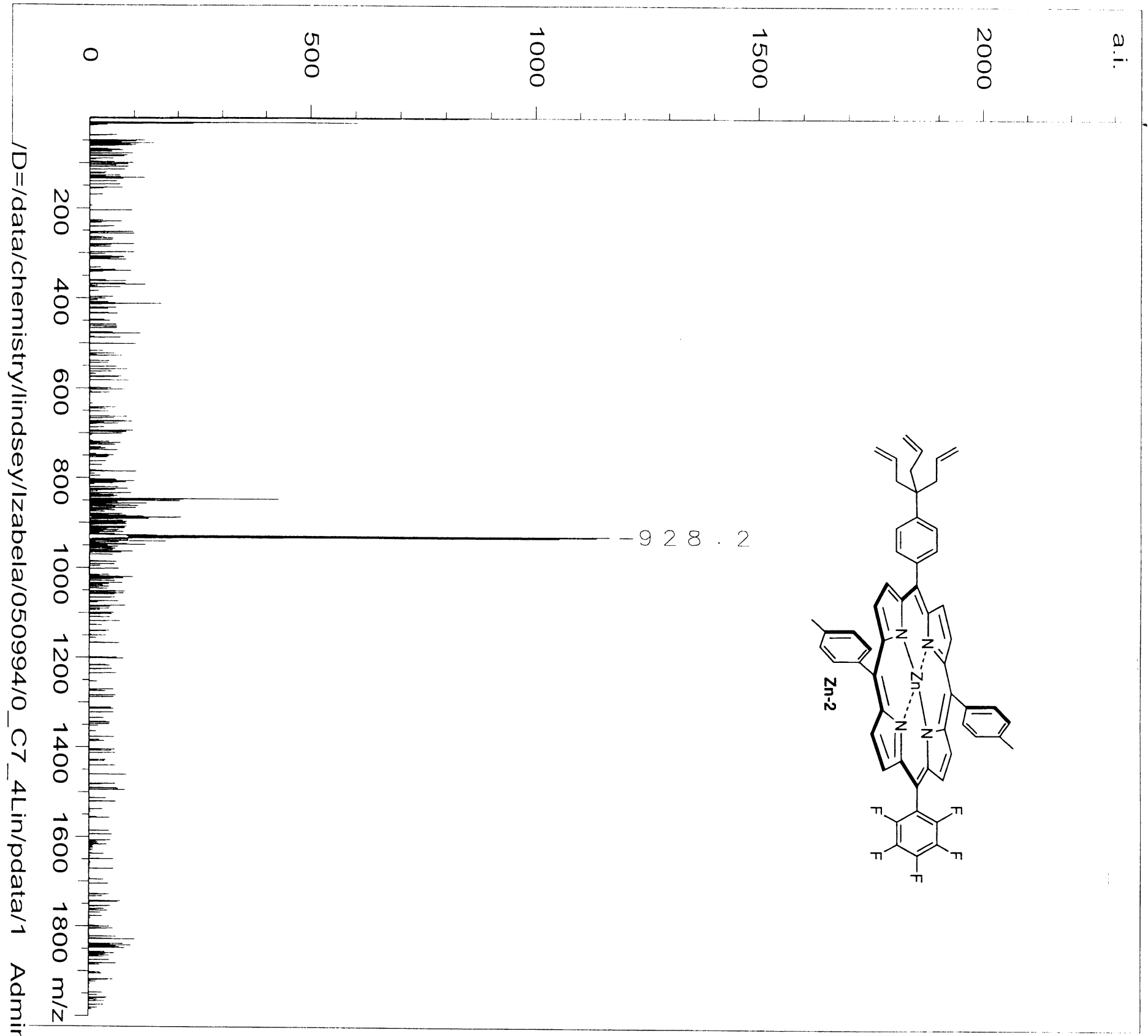

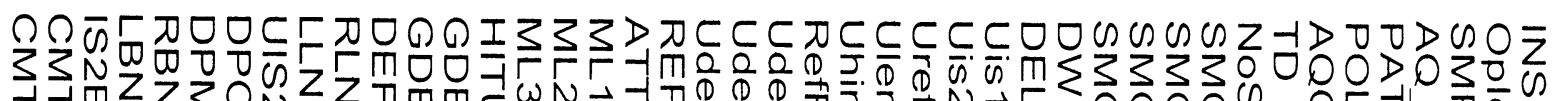

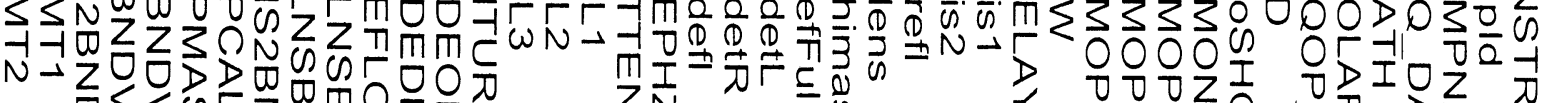

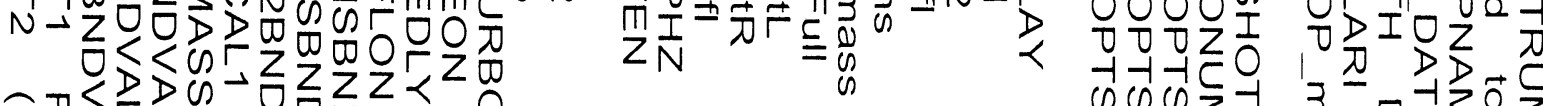

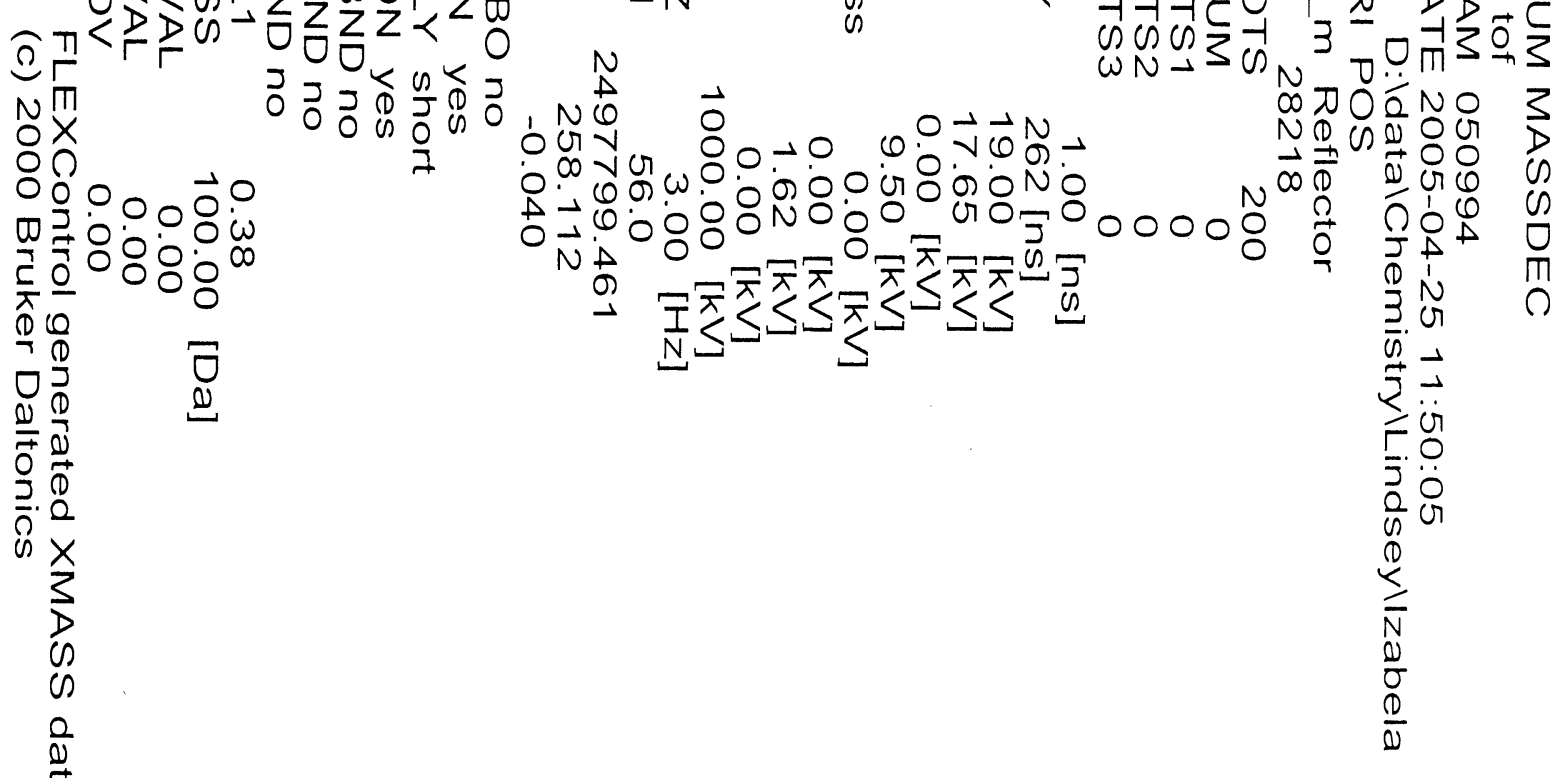




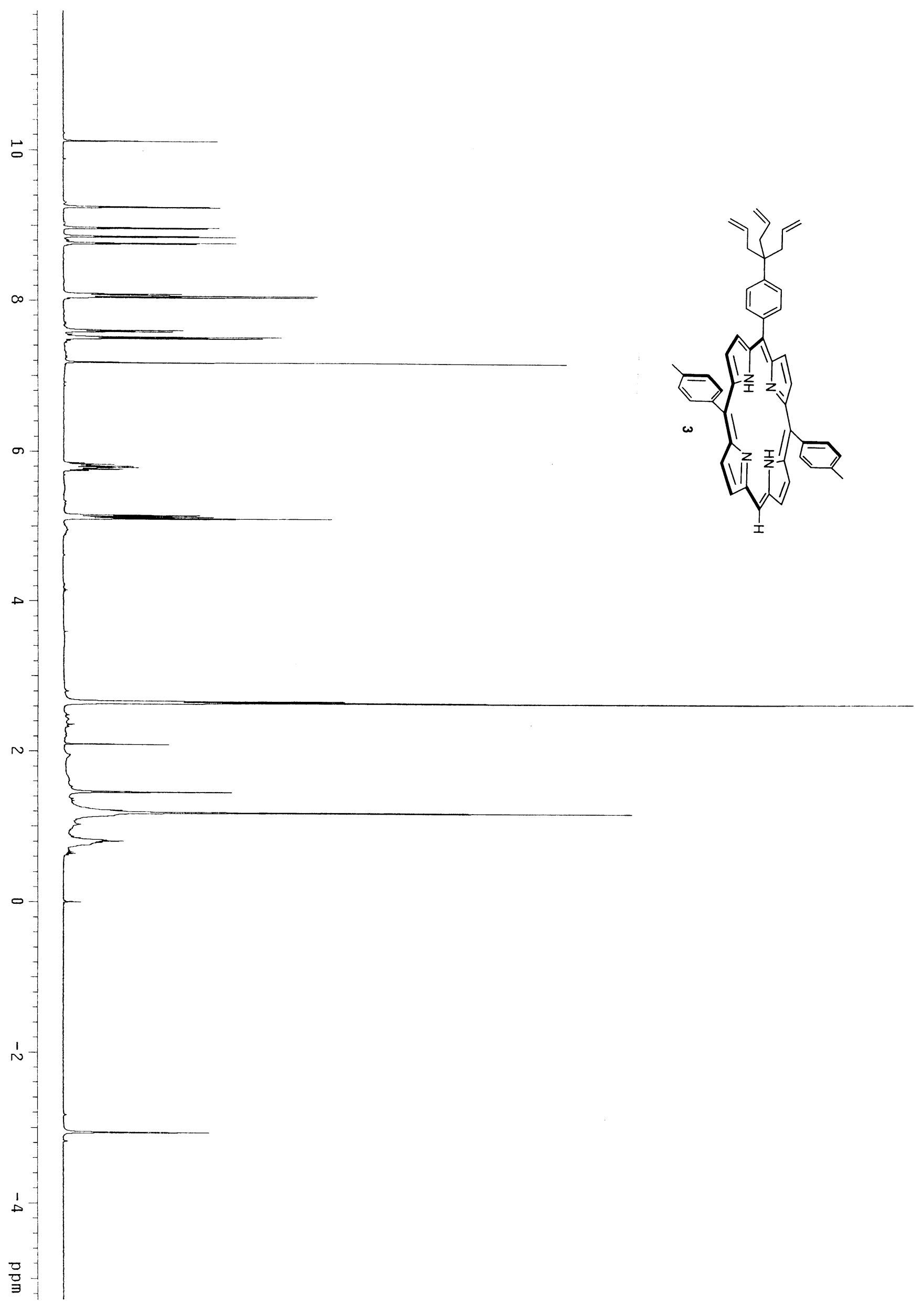



0
용
용

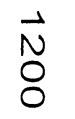
索

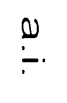
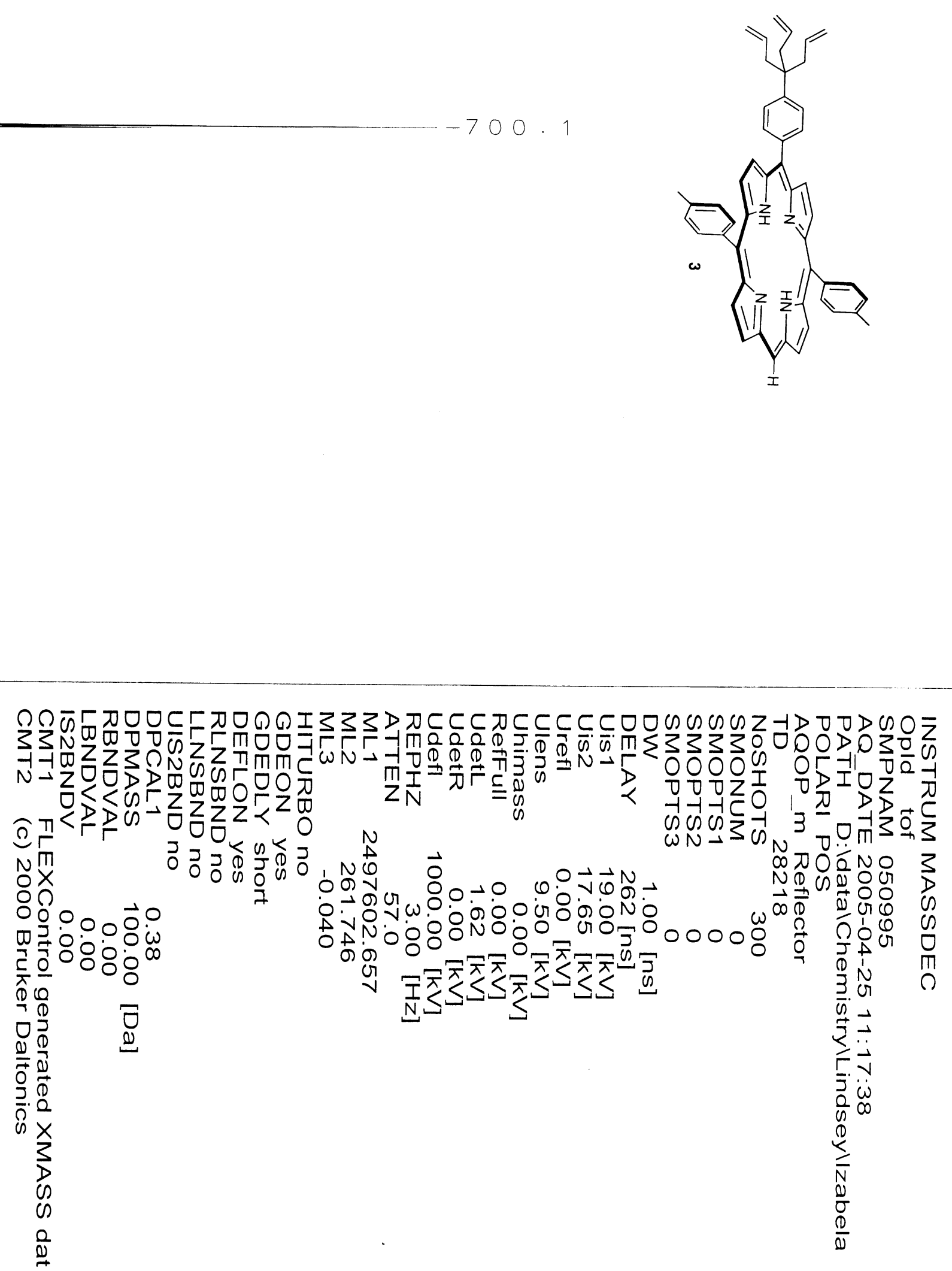


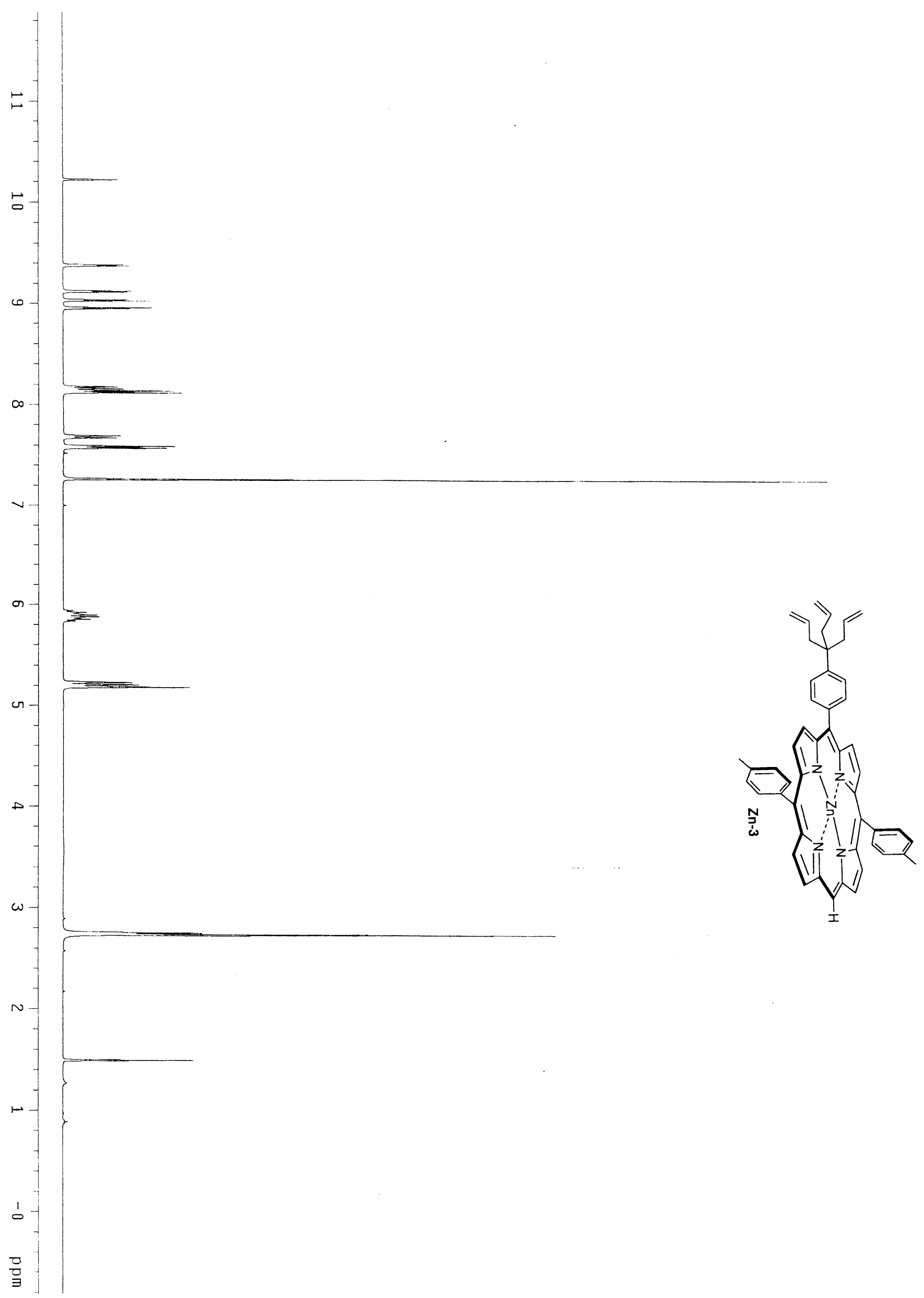




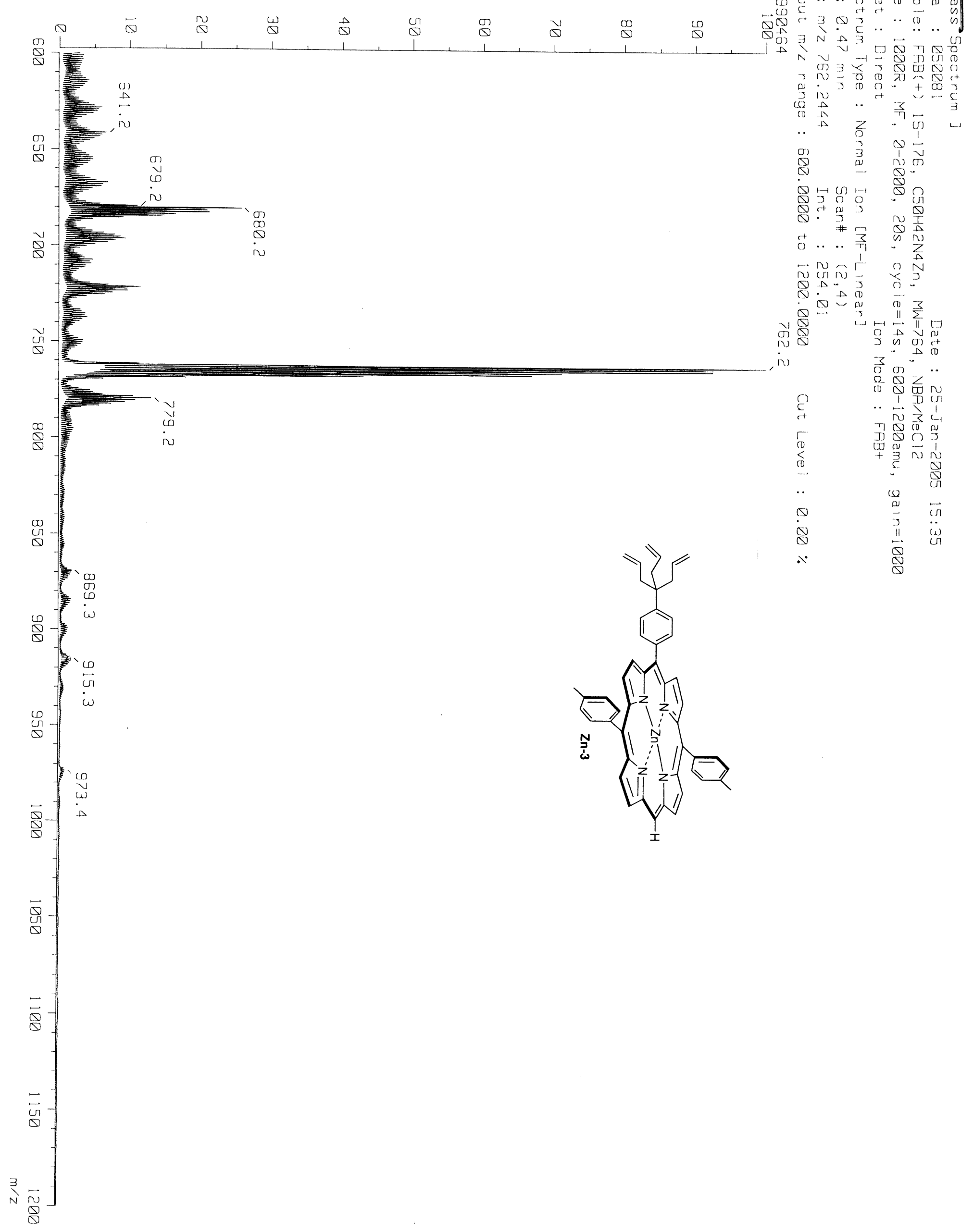




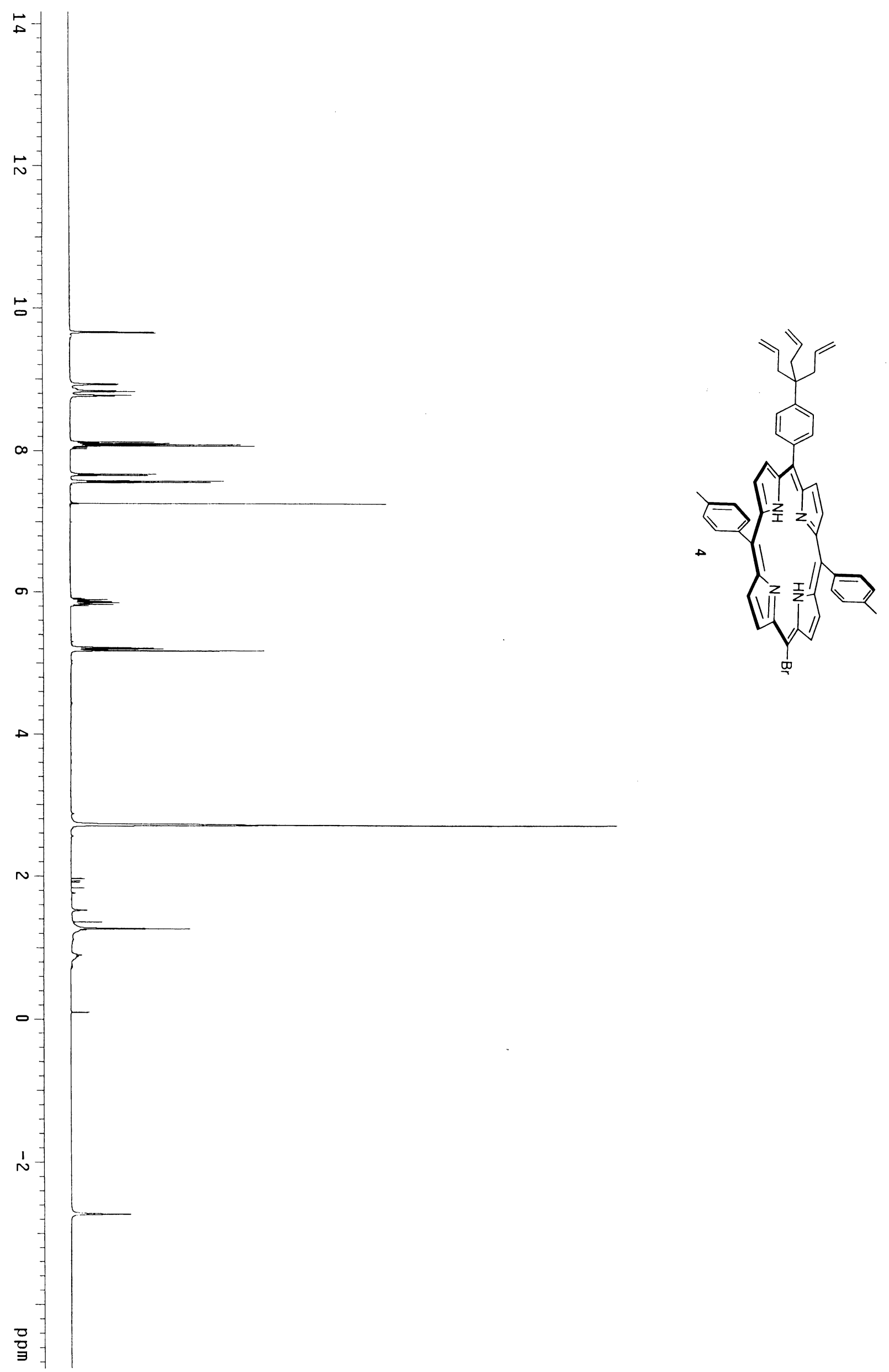




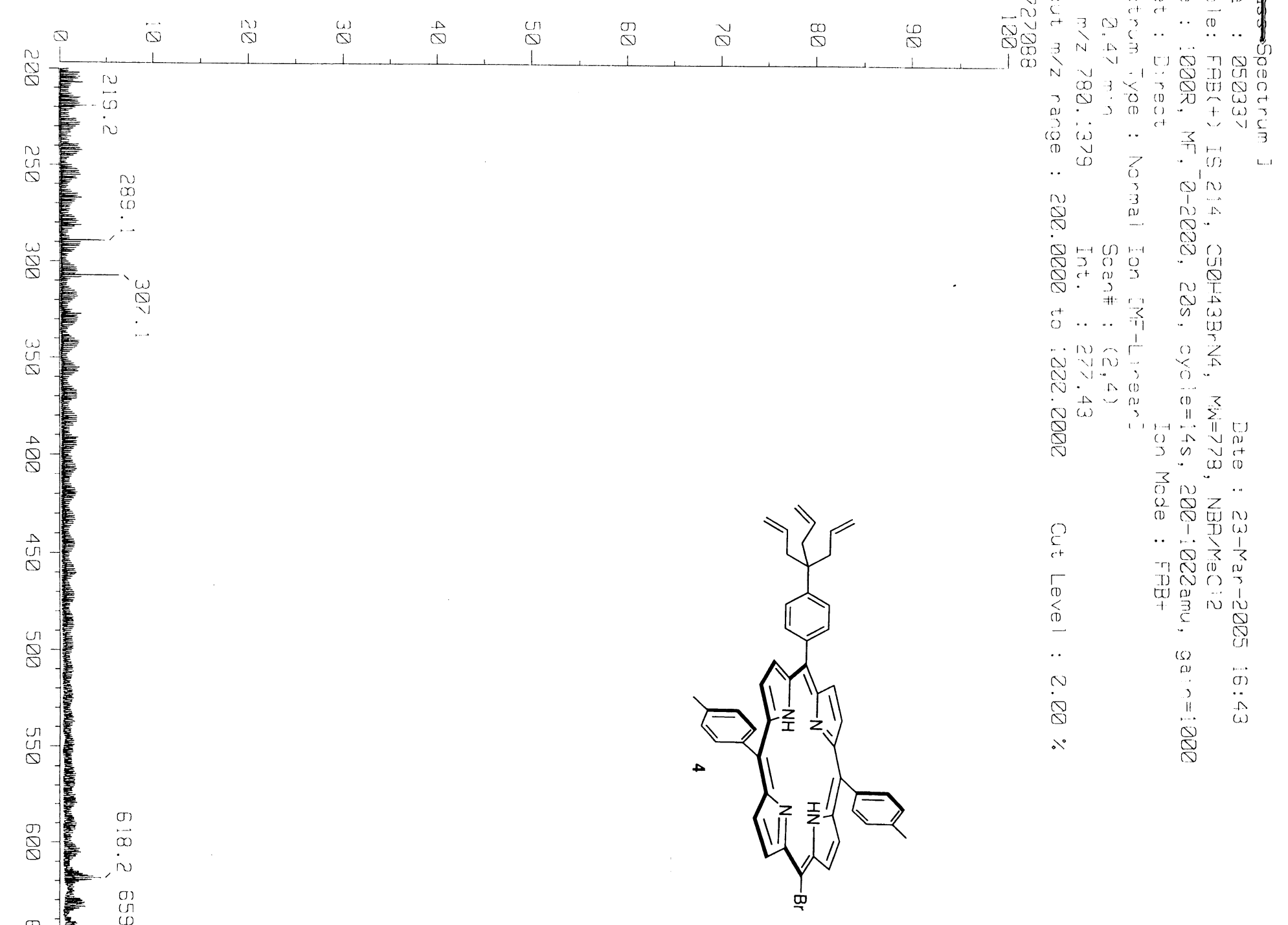




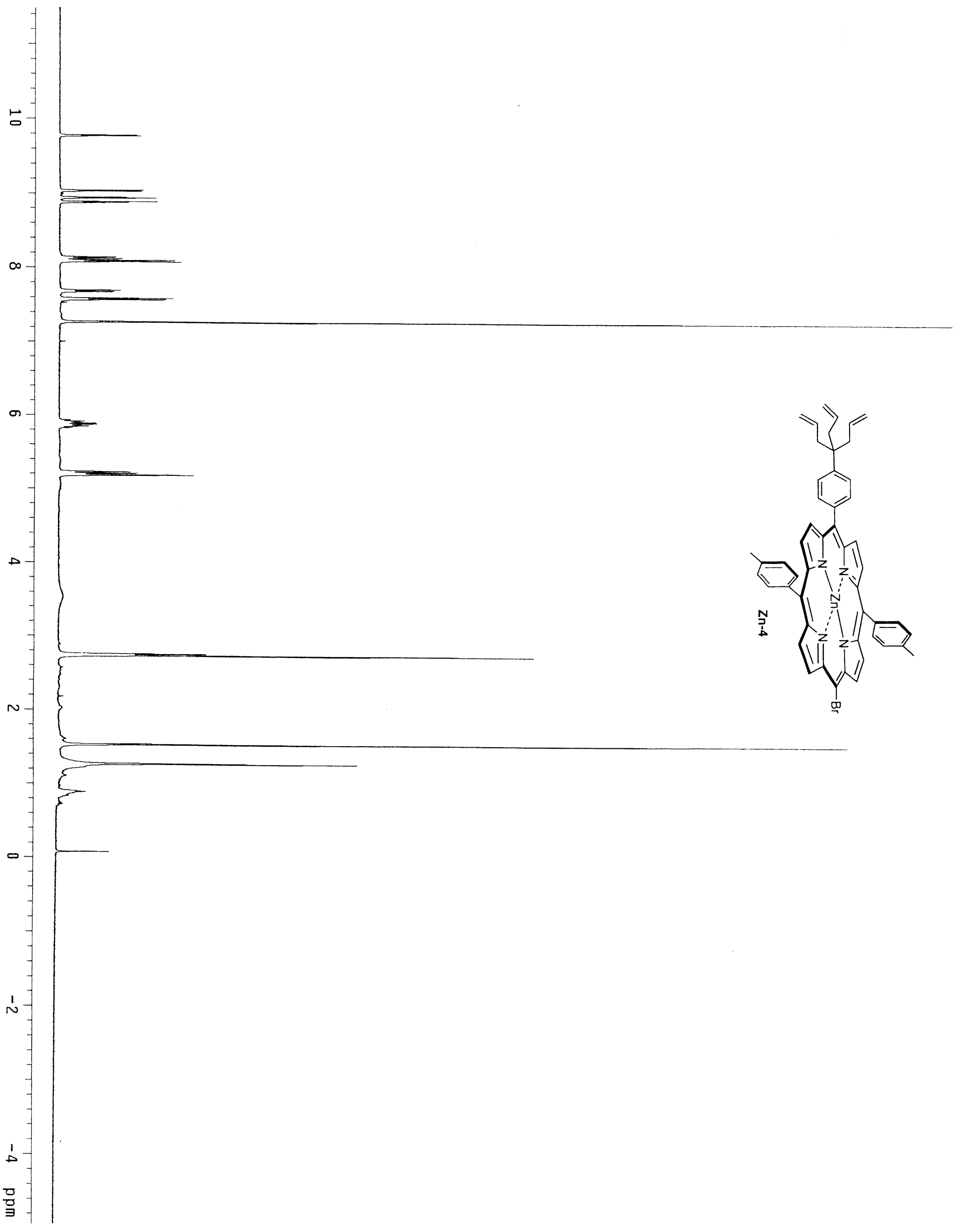




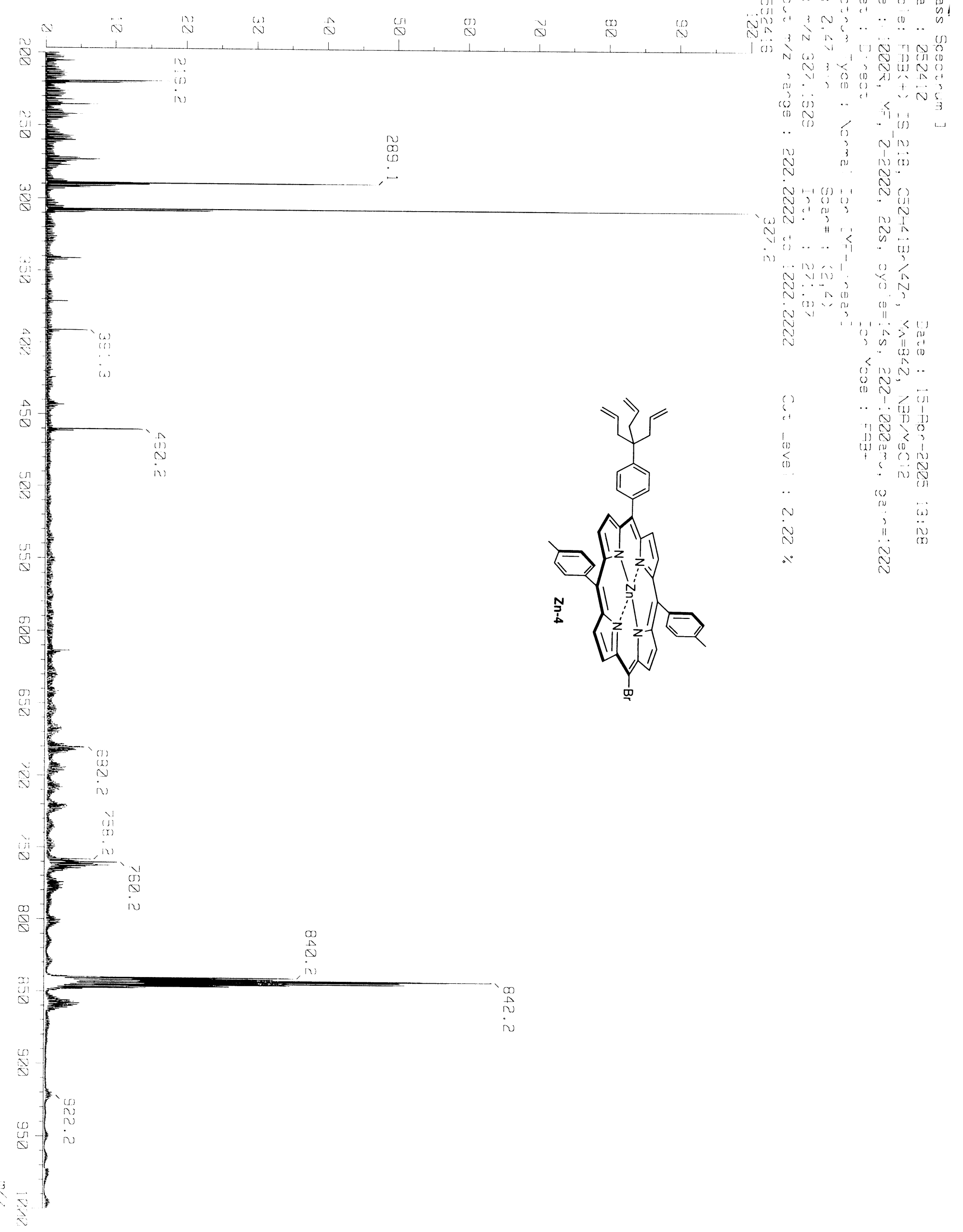




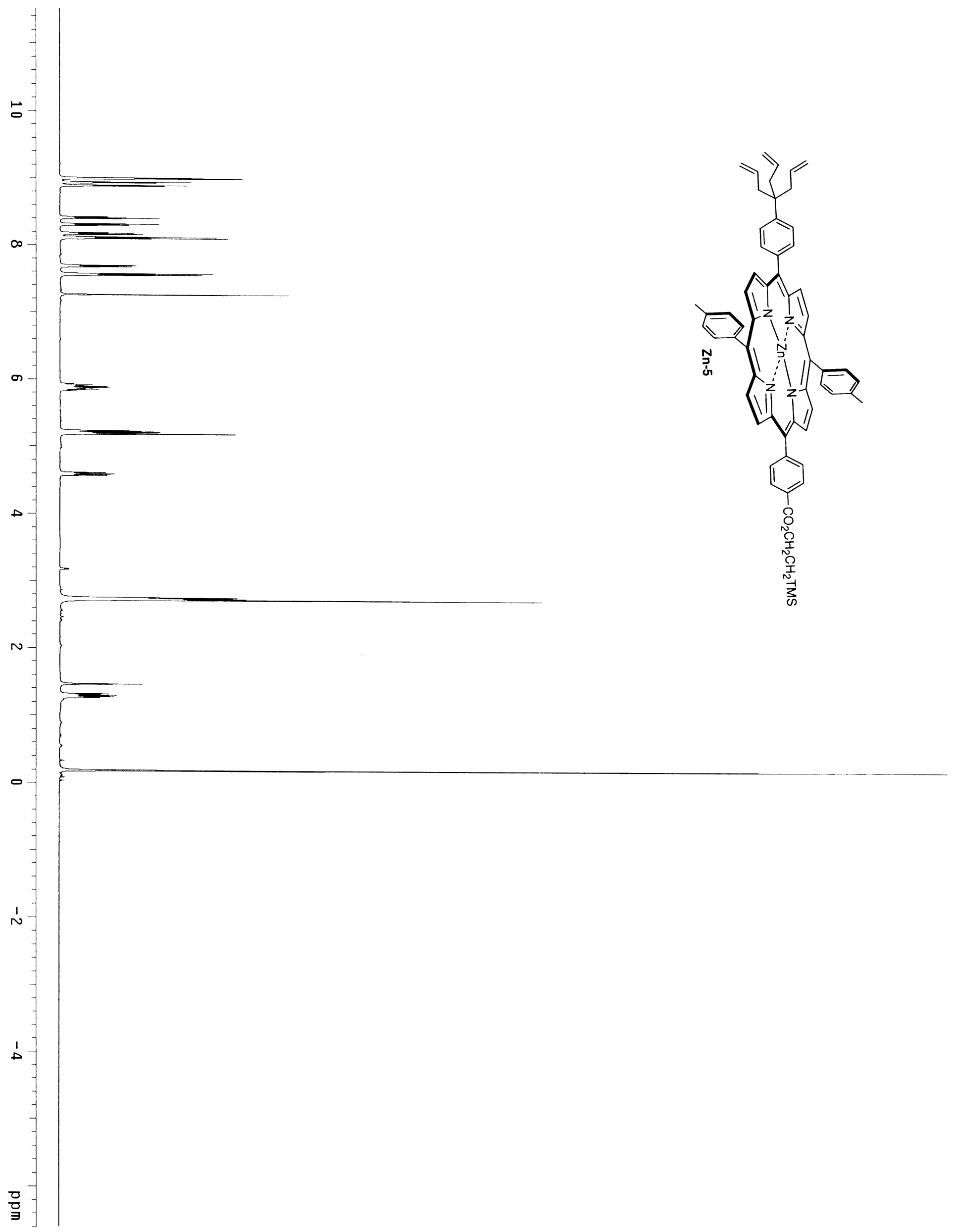




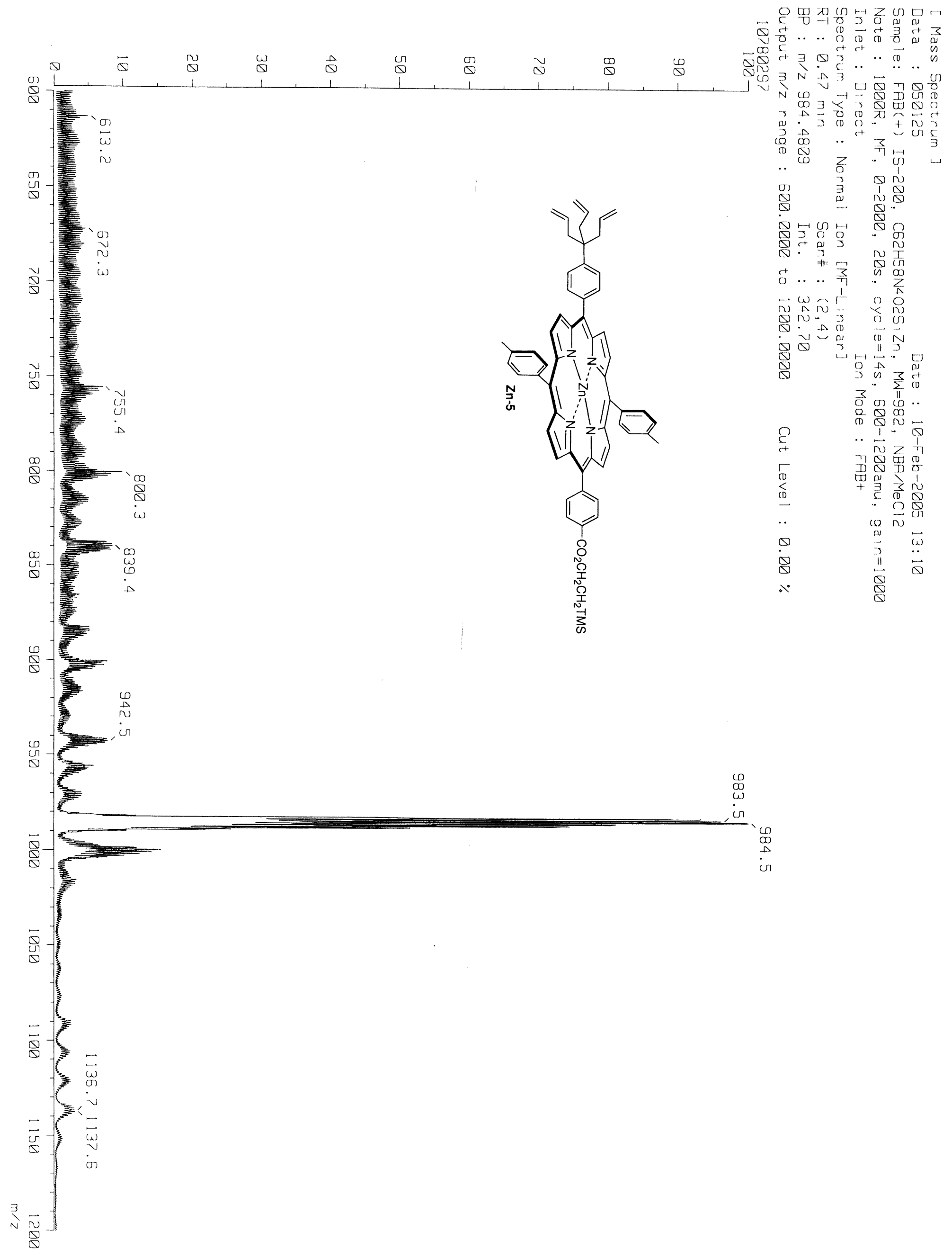




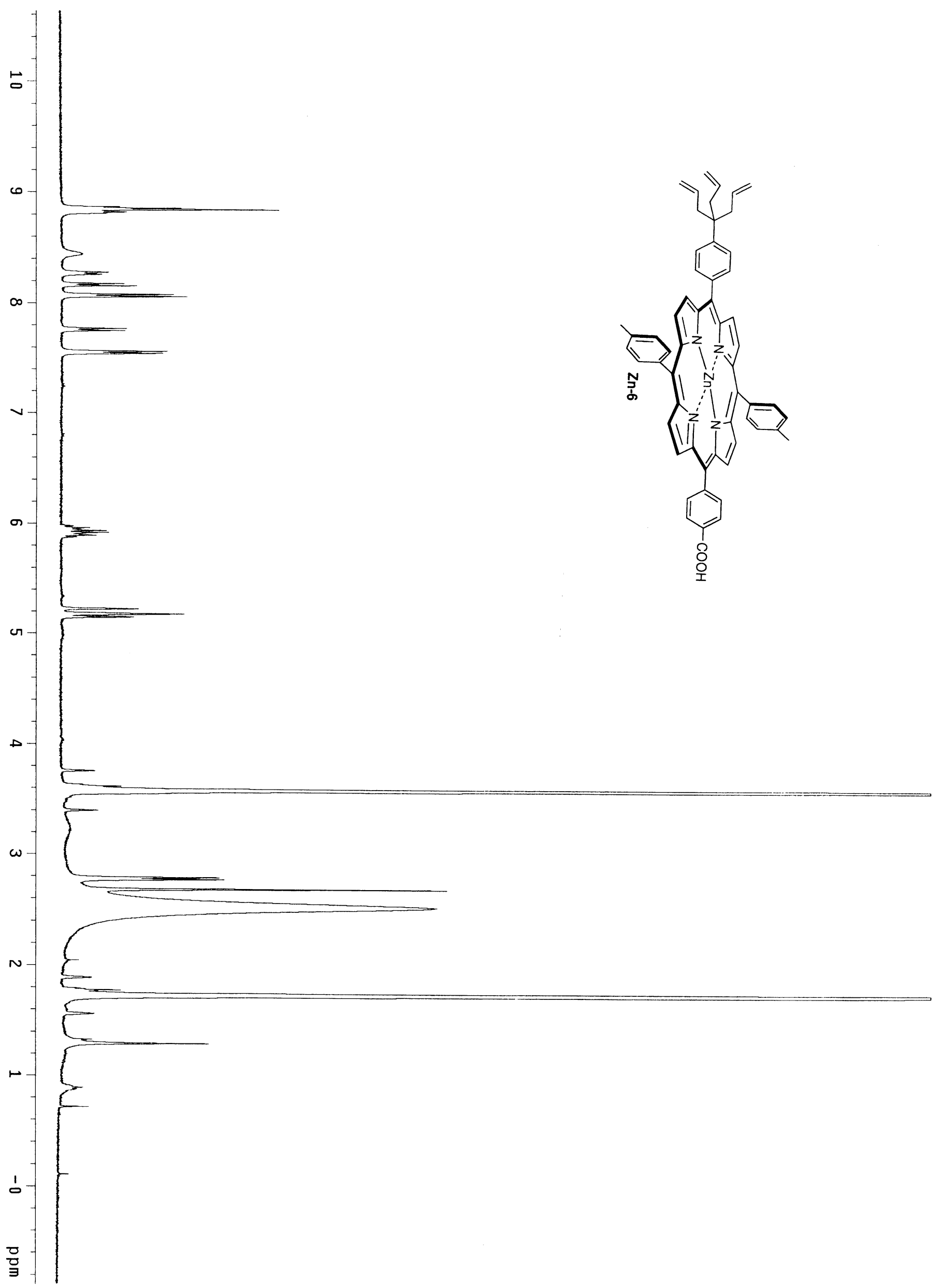




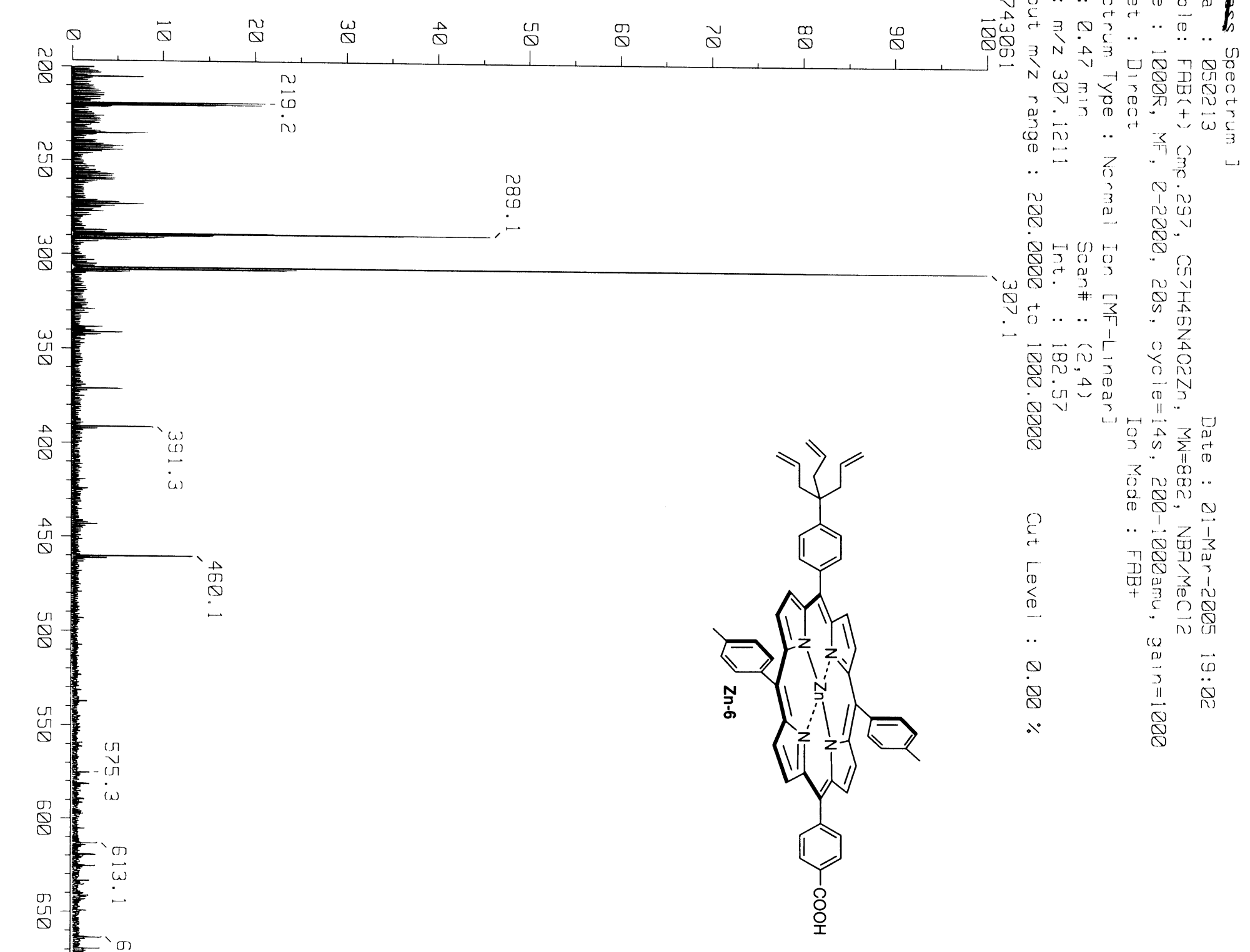




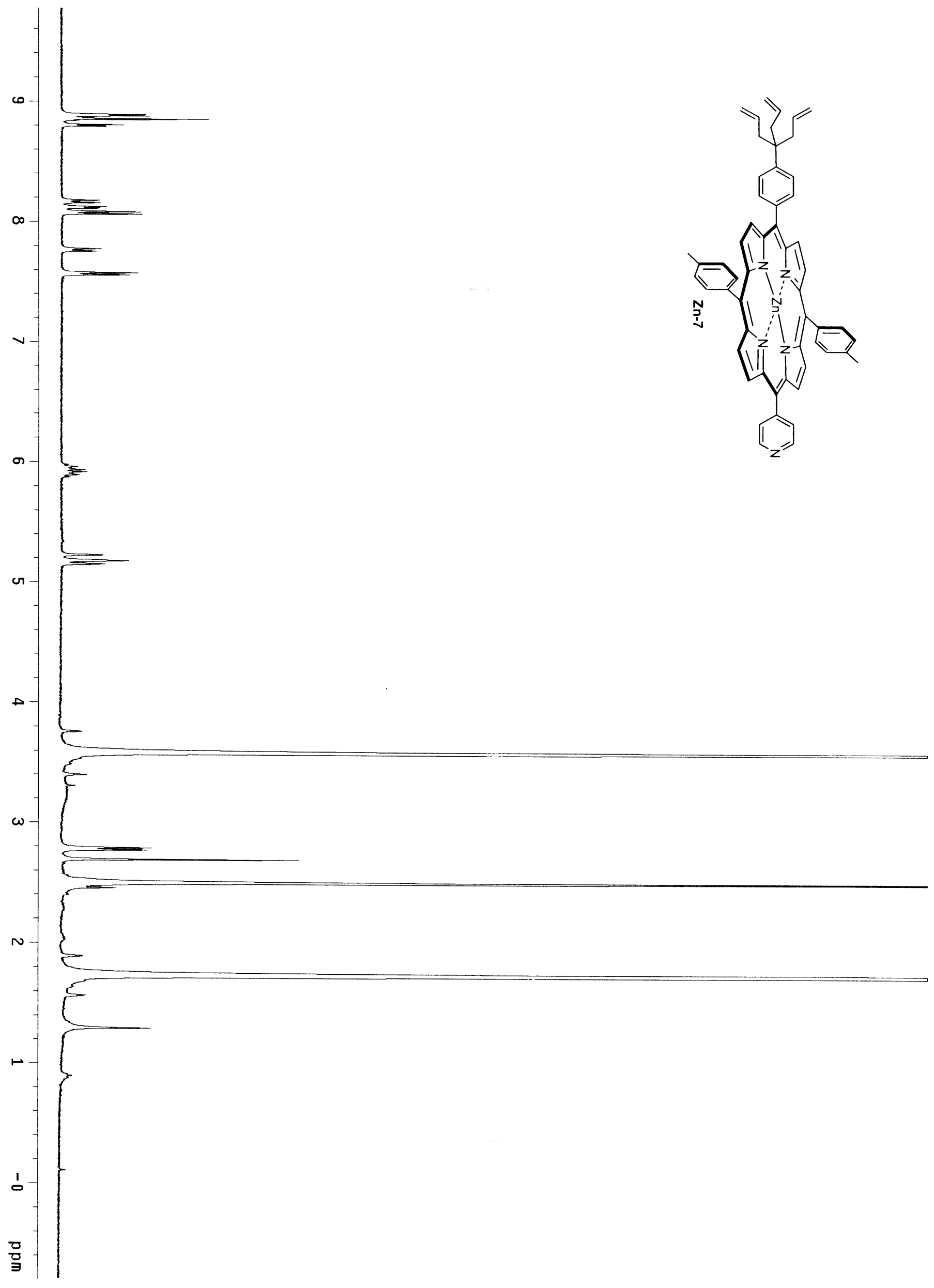




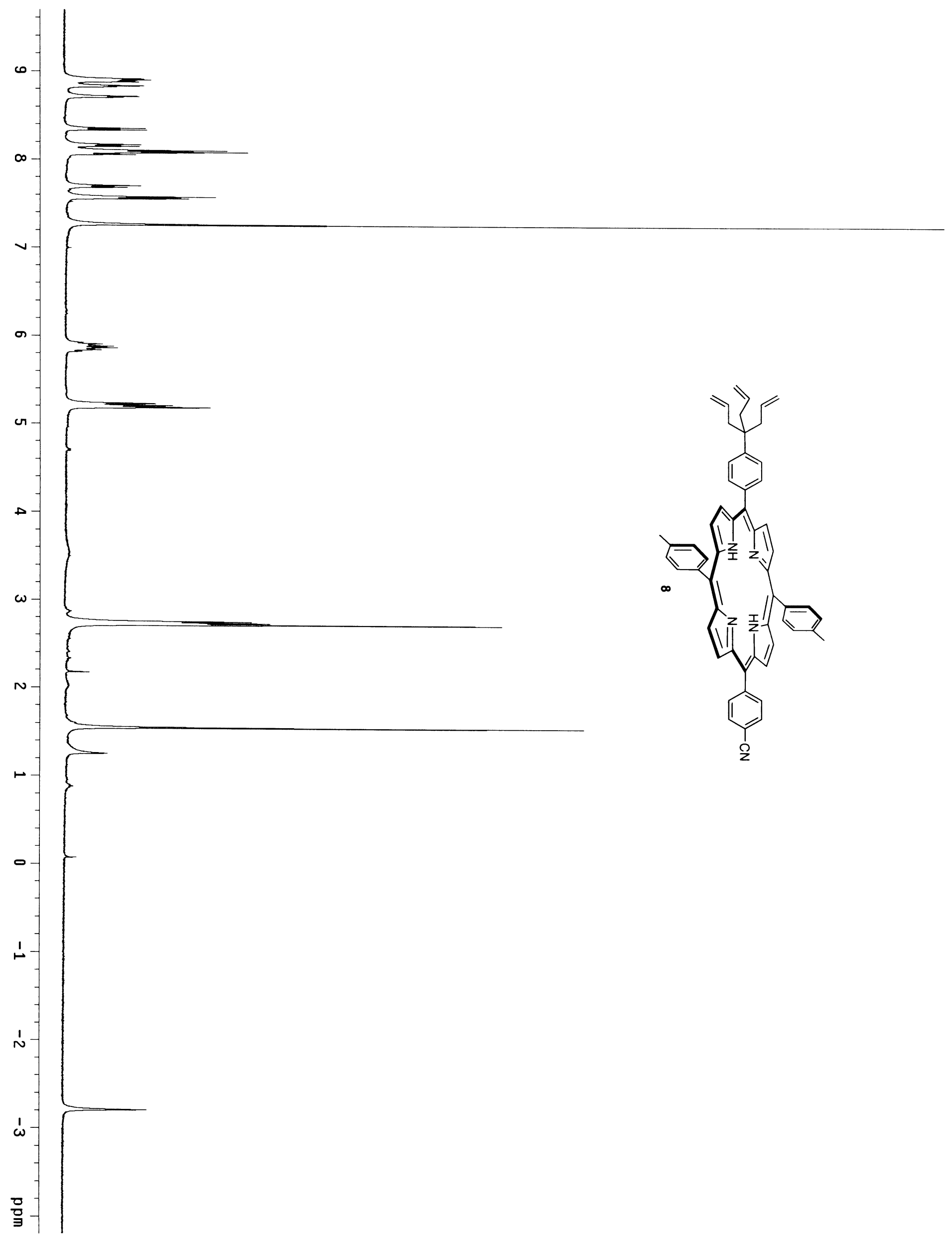




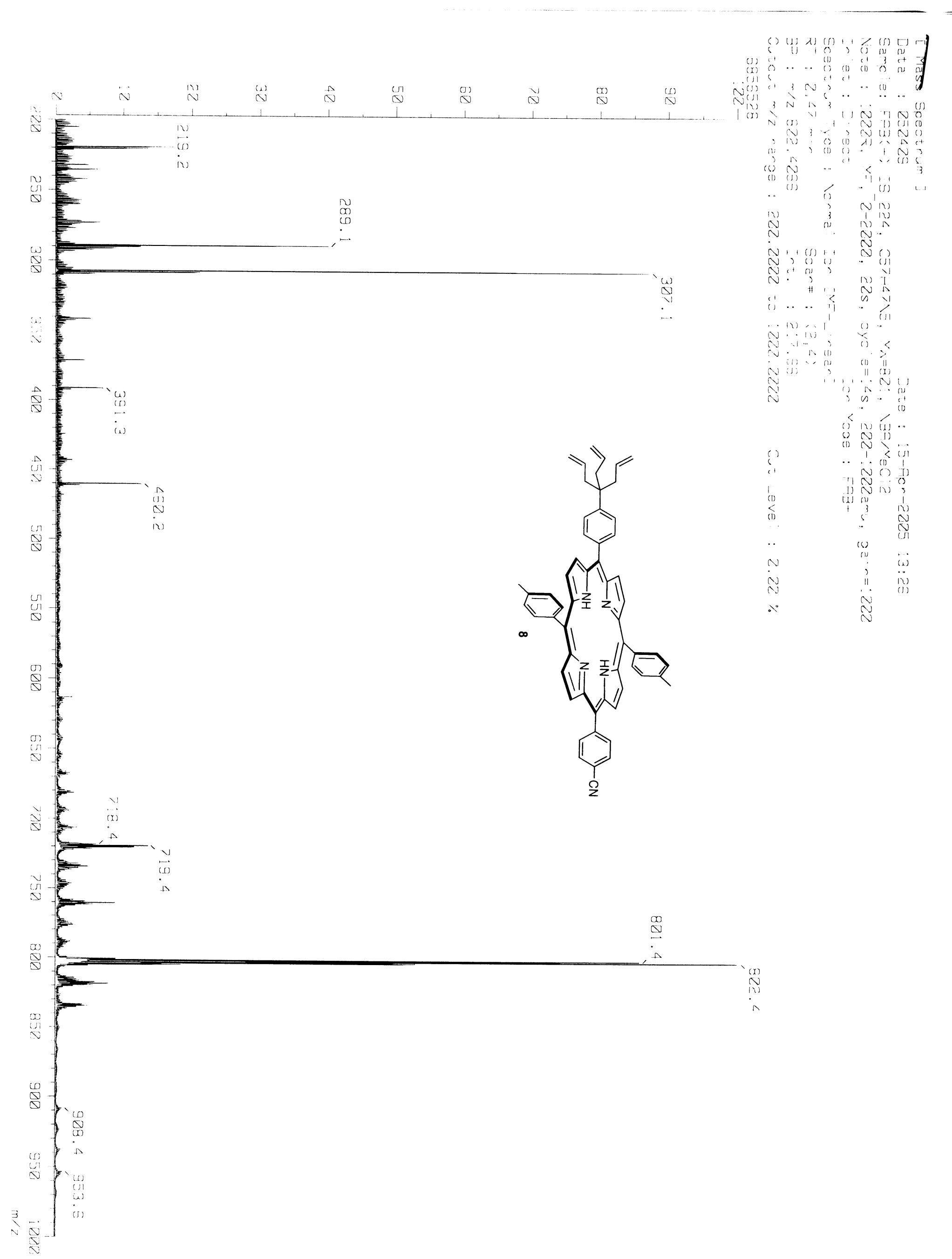




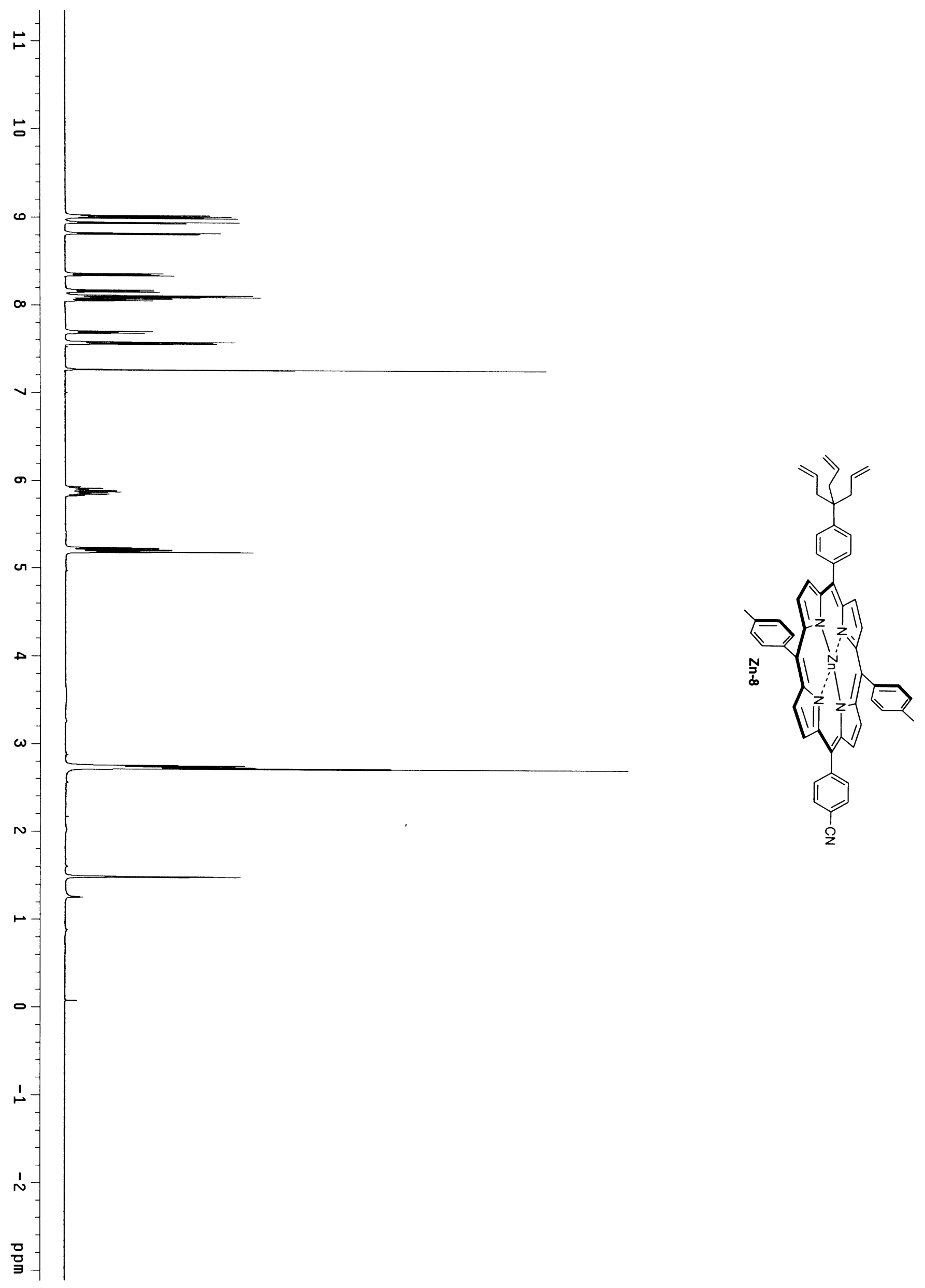




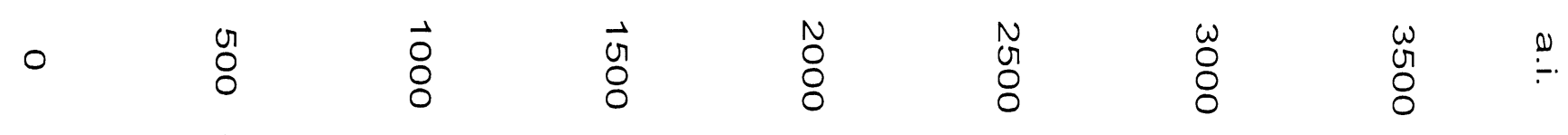

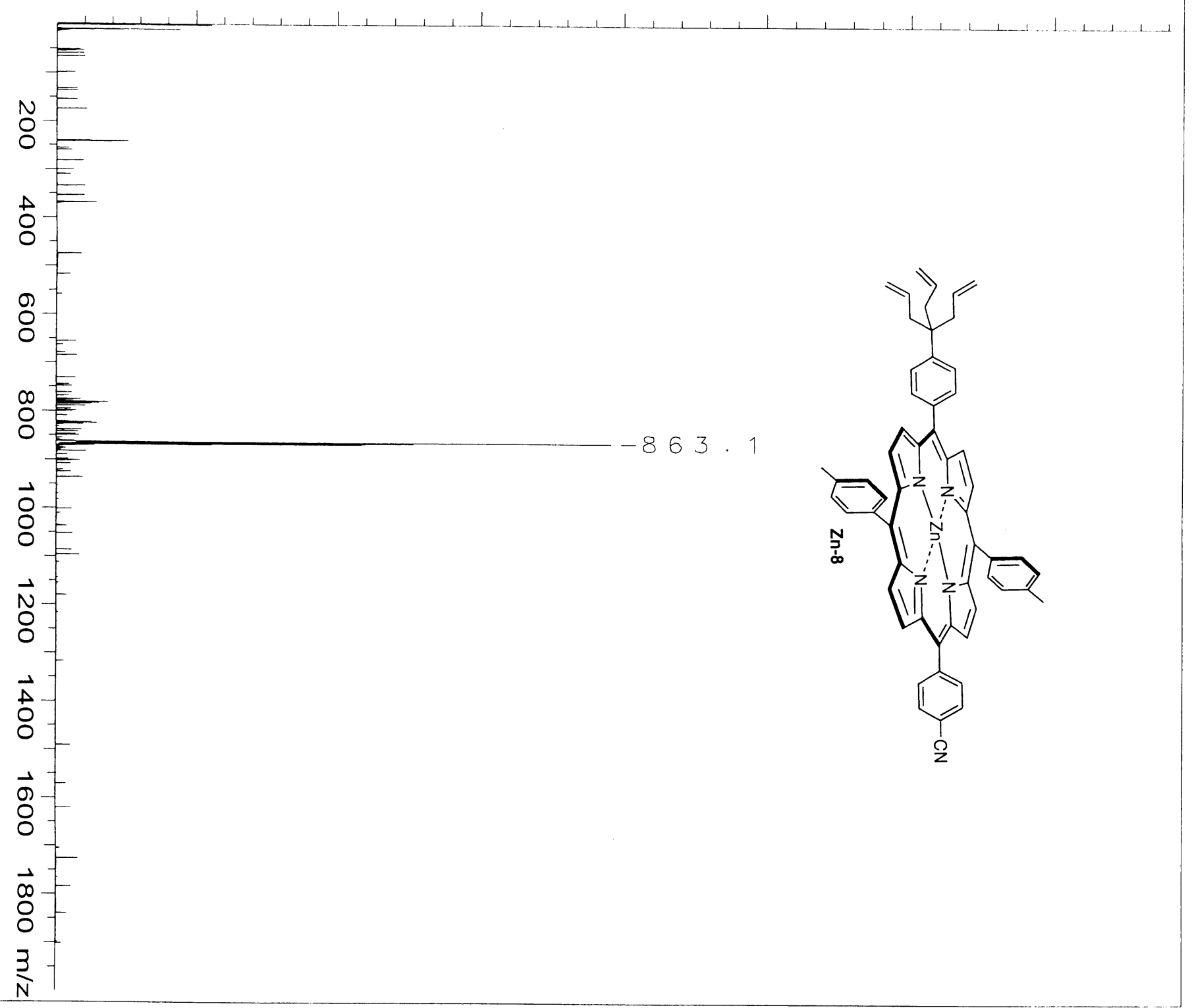

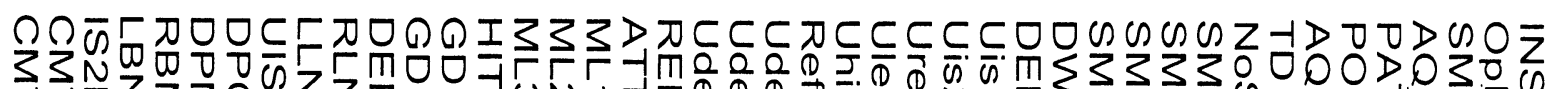

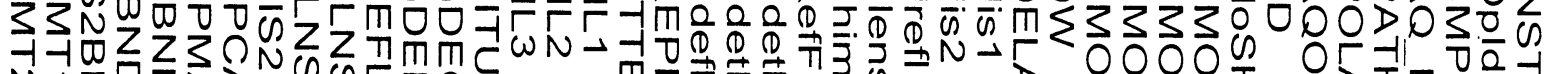

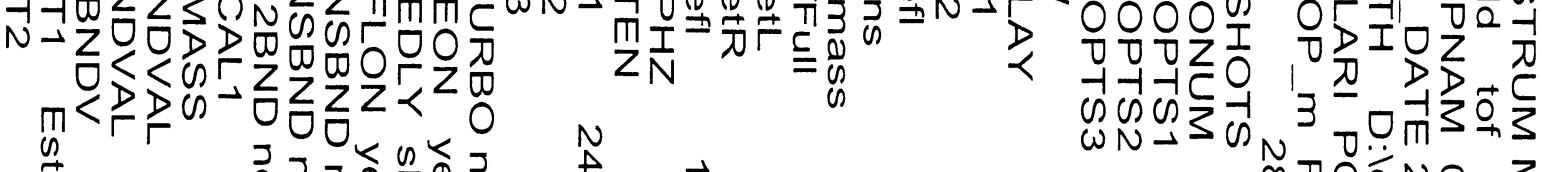

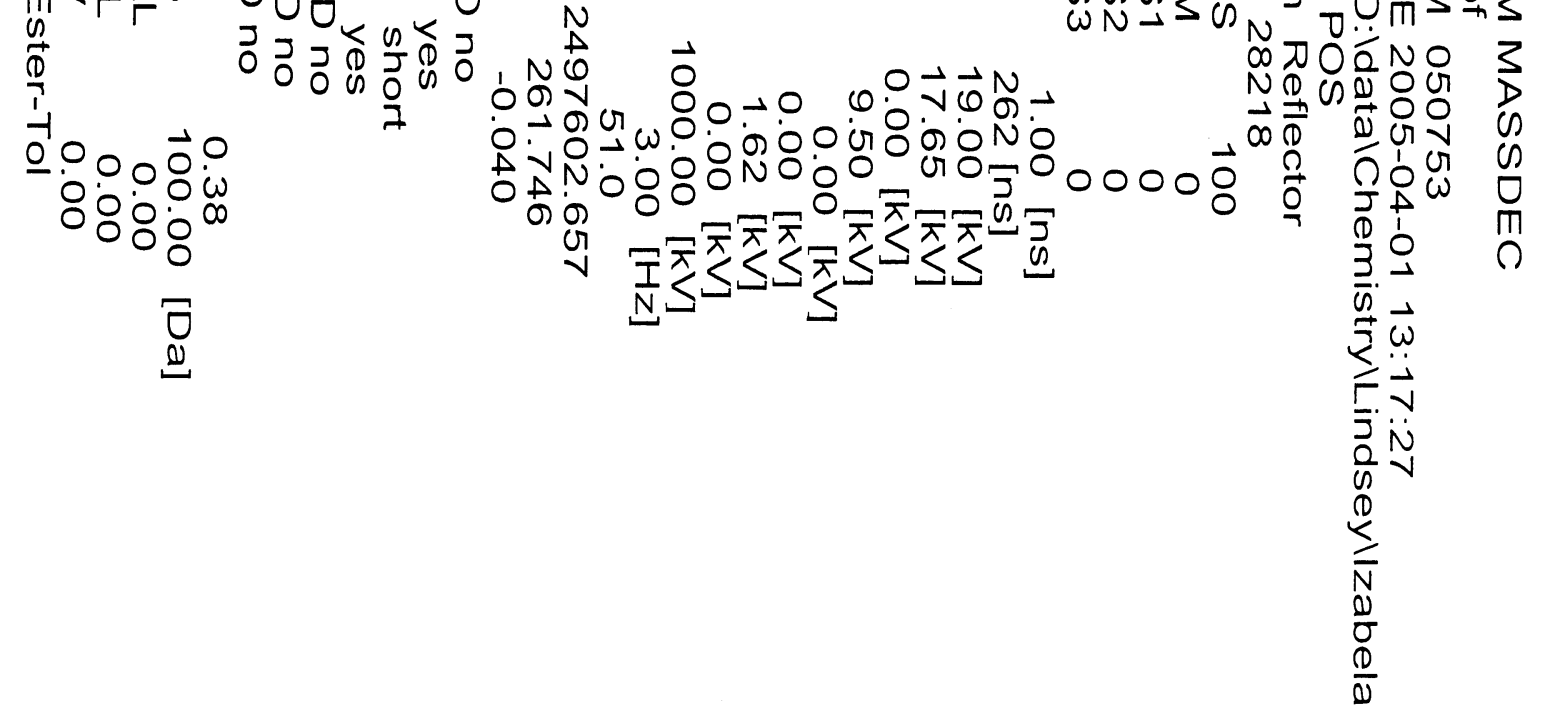




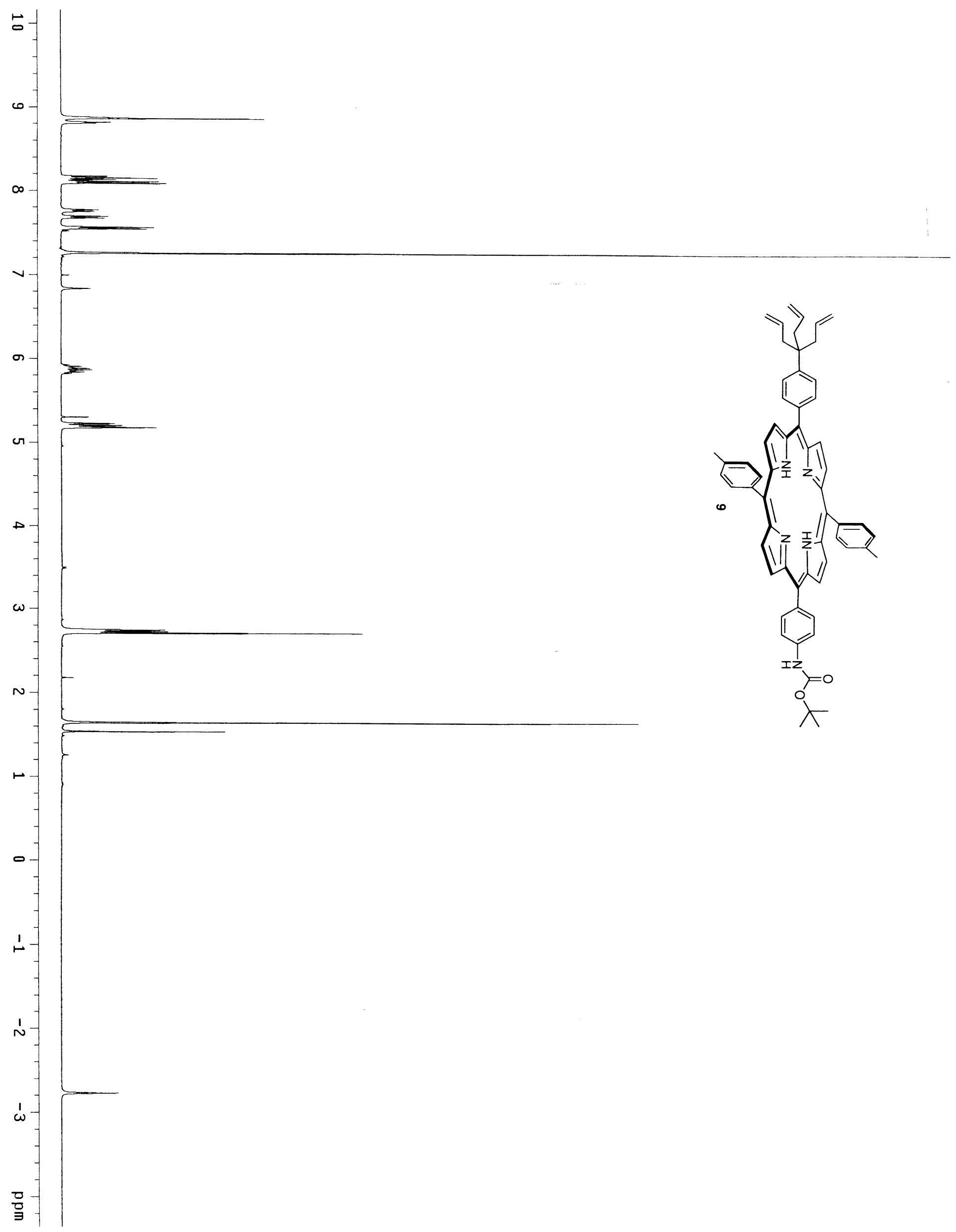




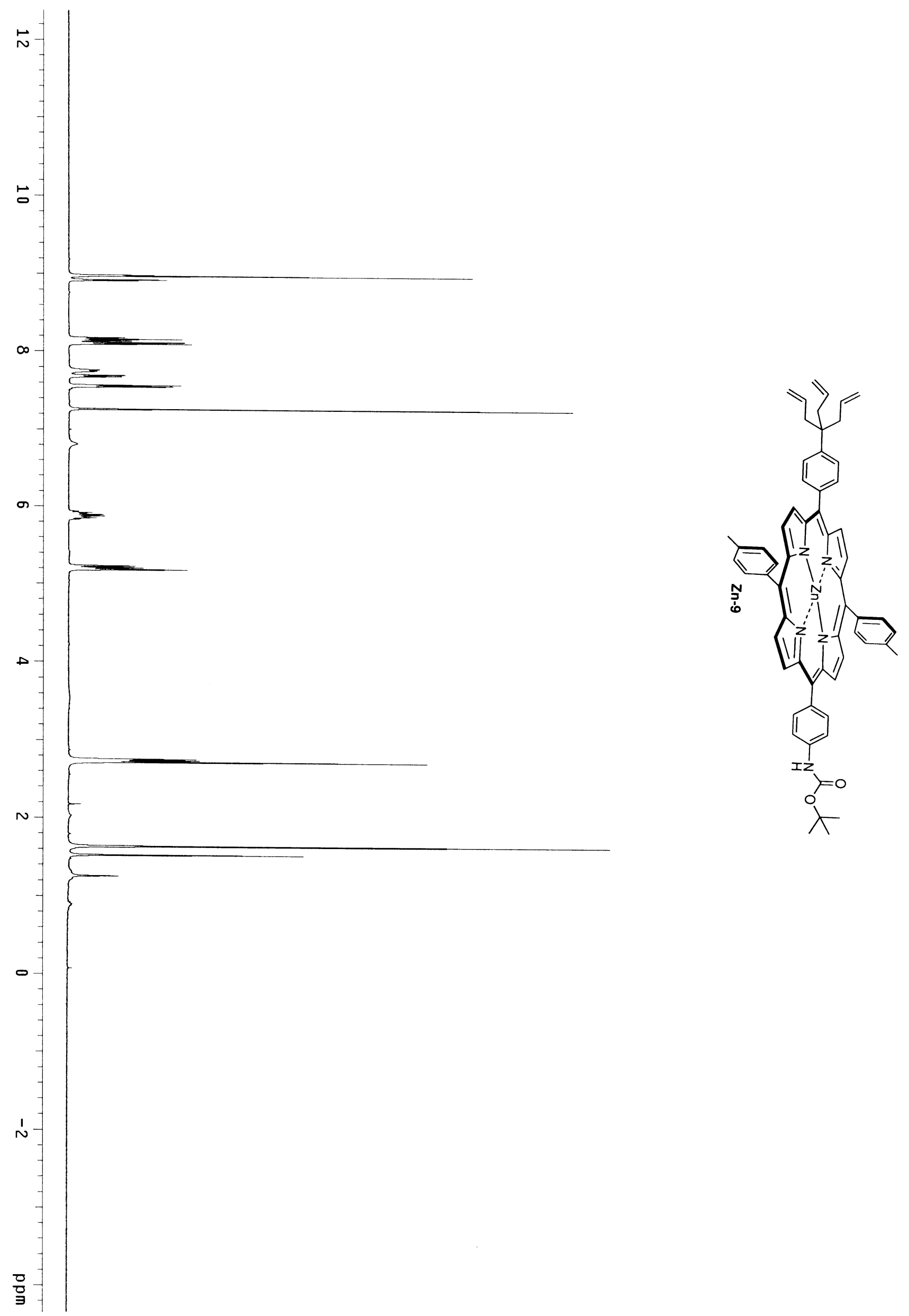




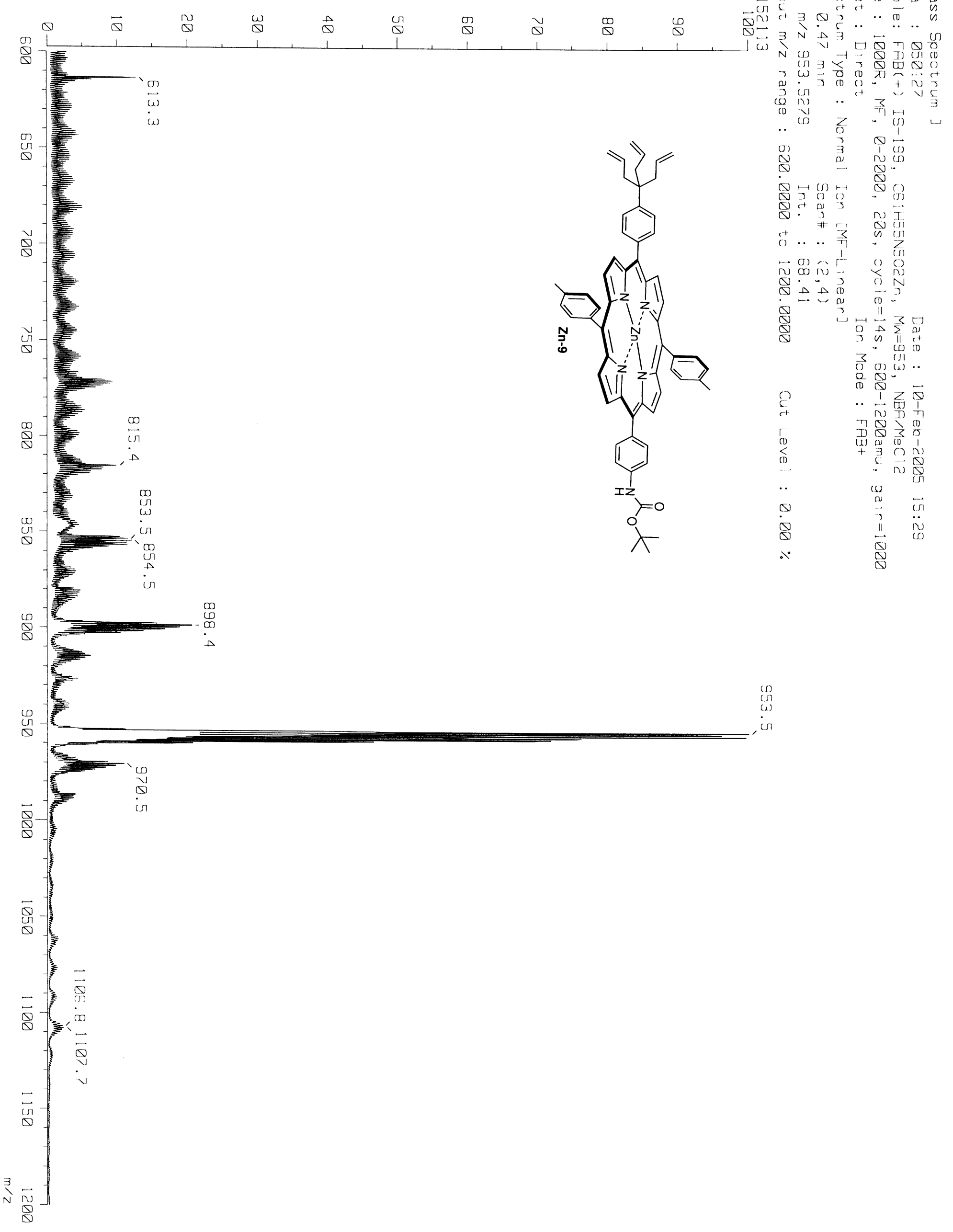




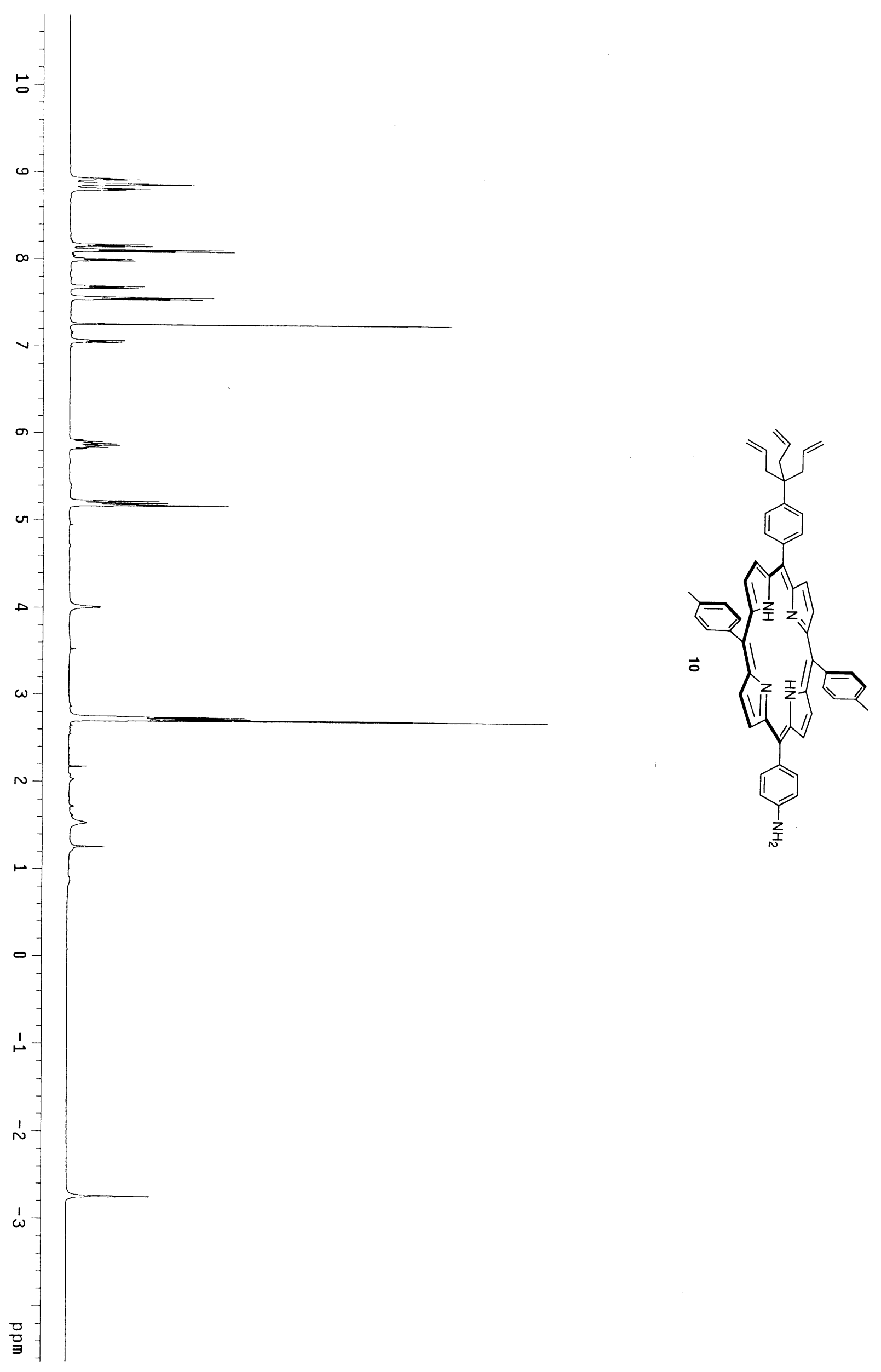




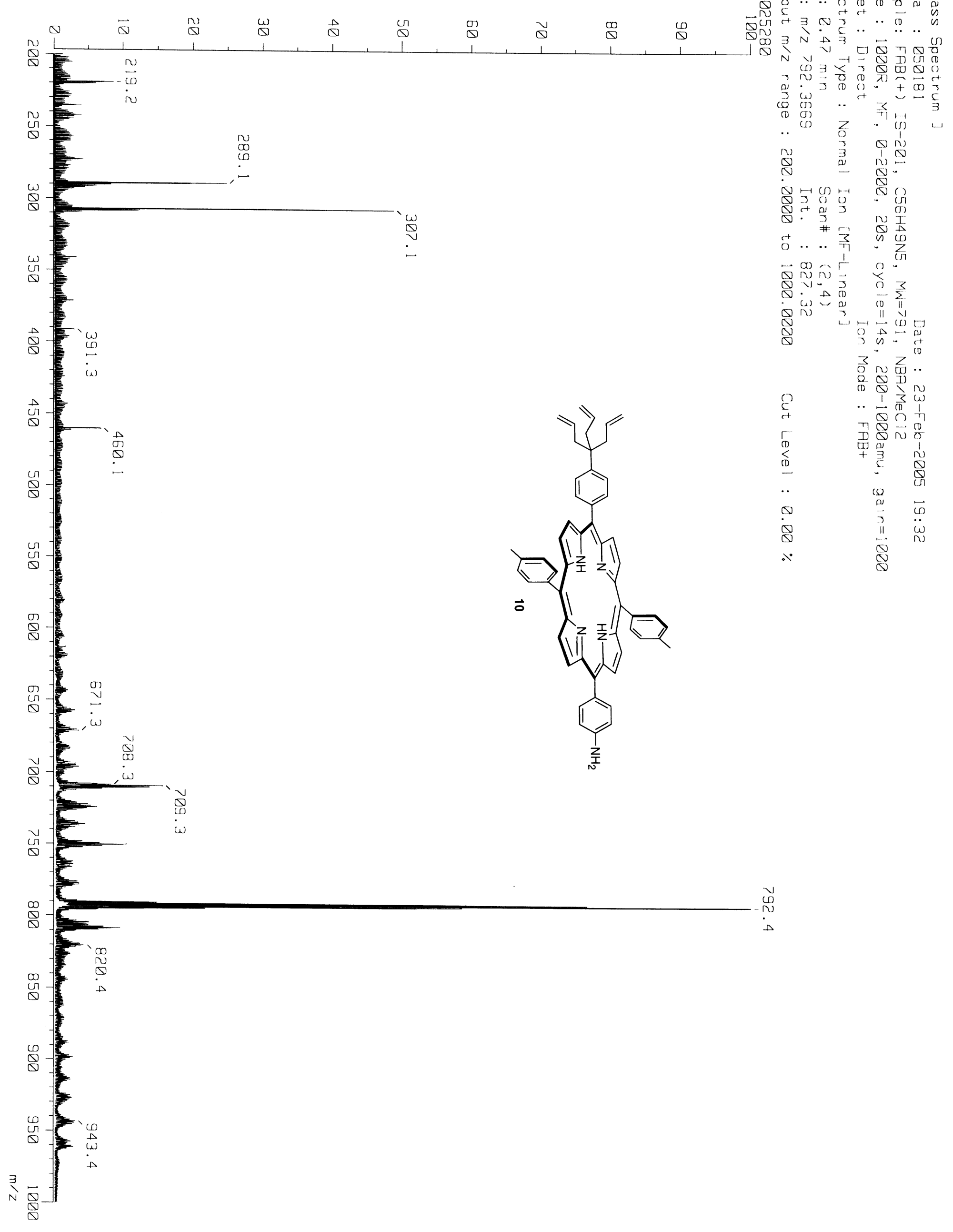




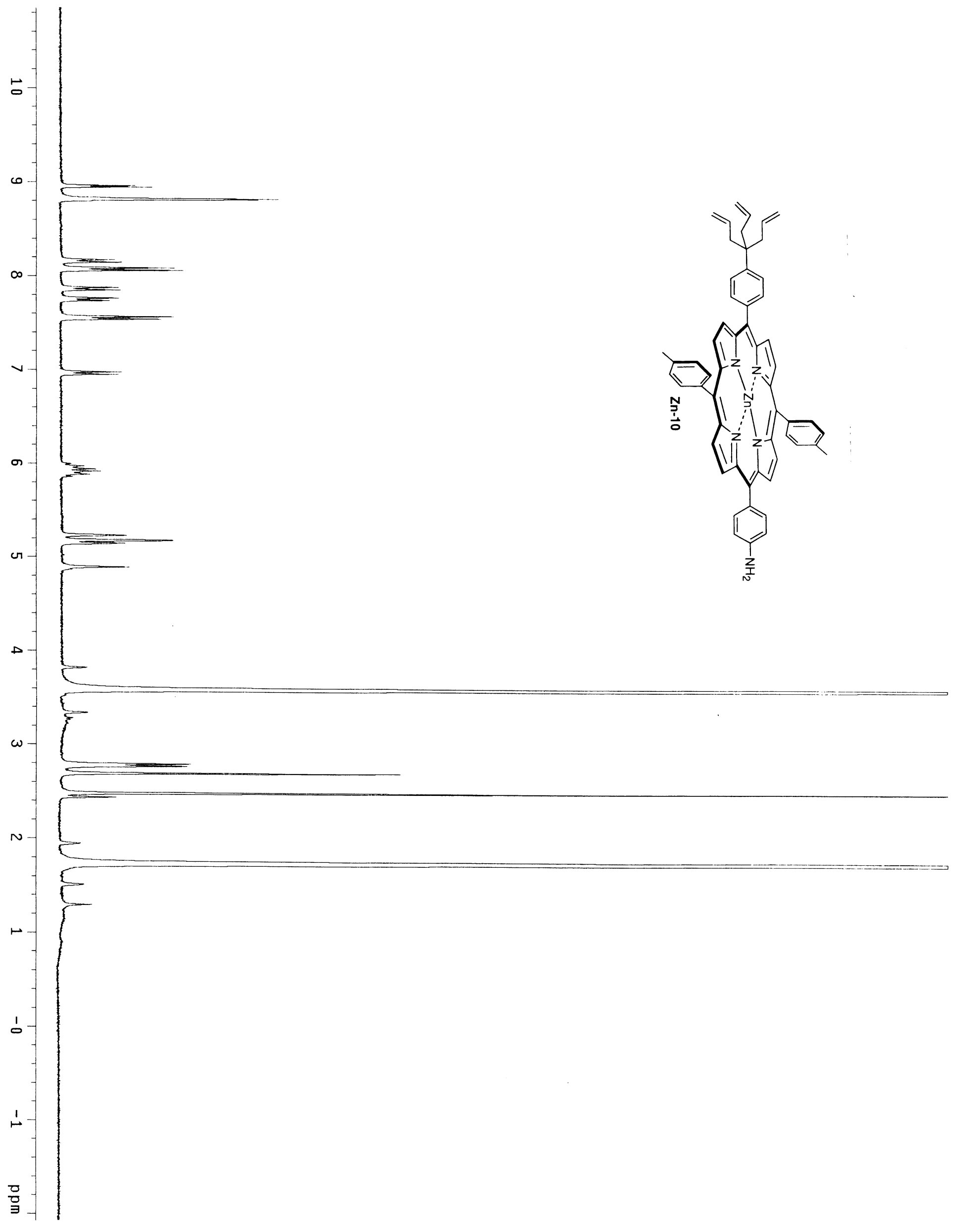



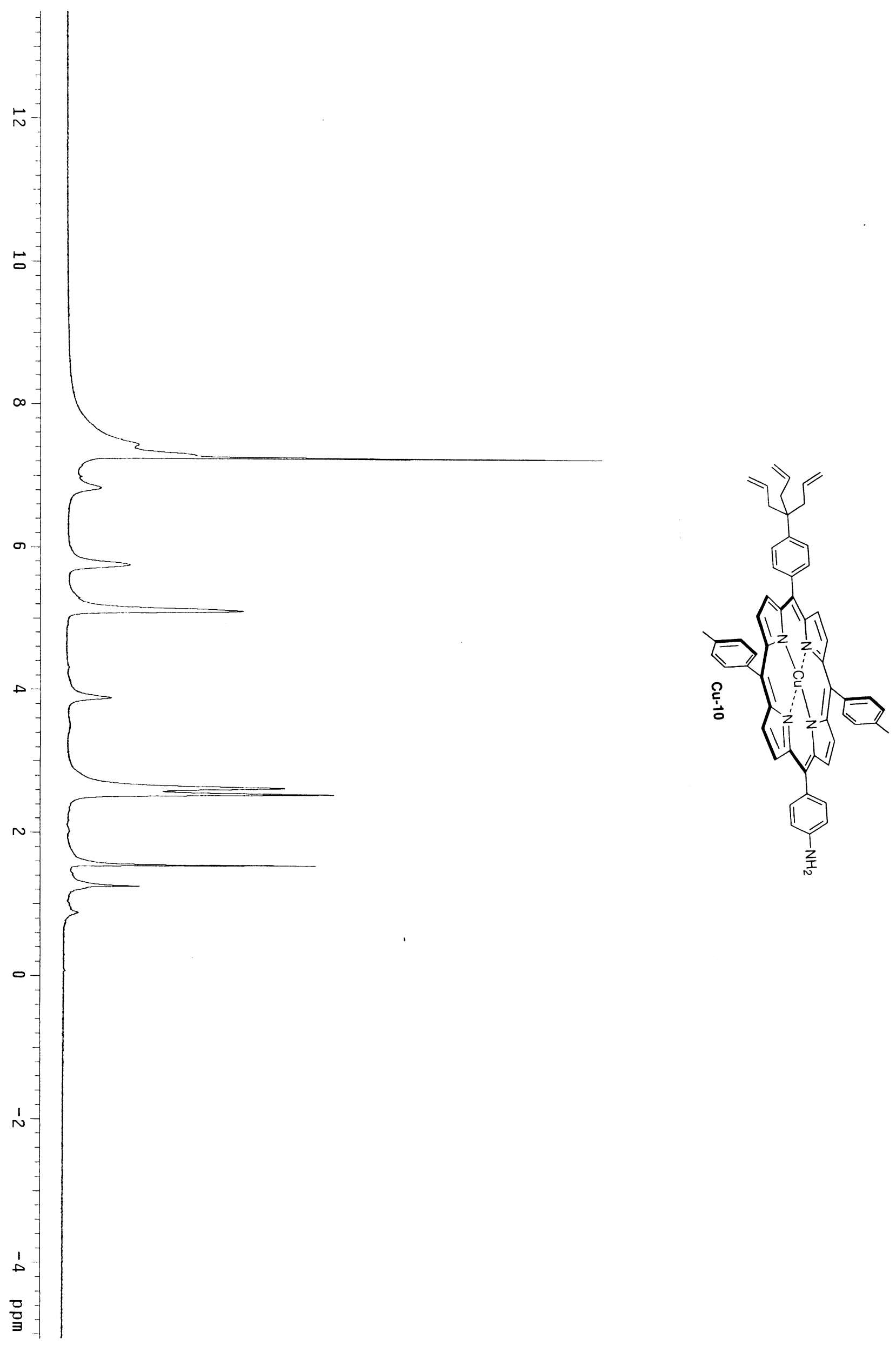


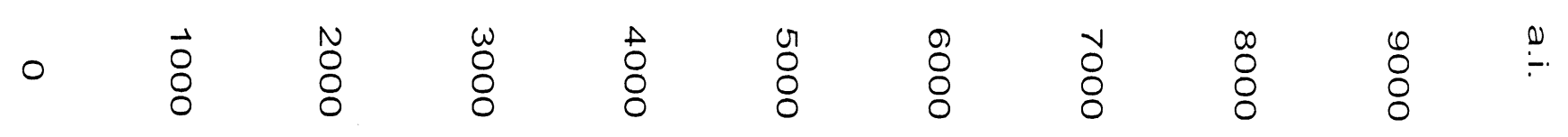

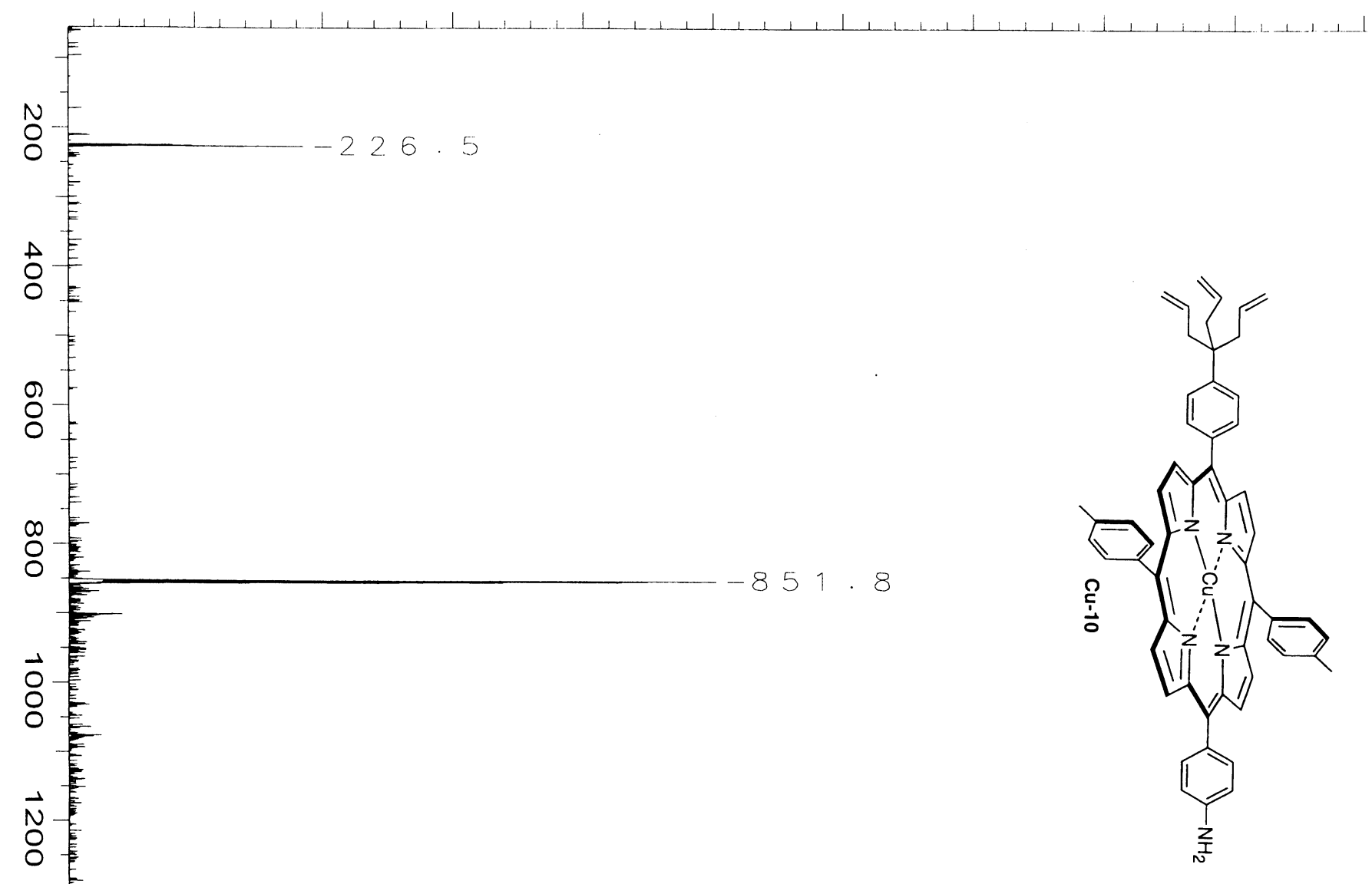

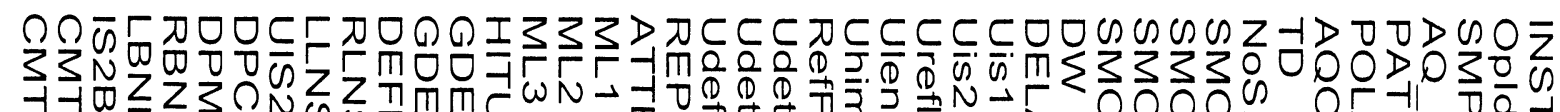

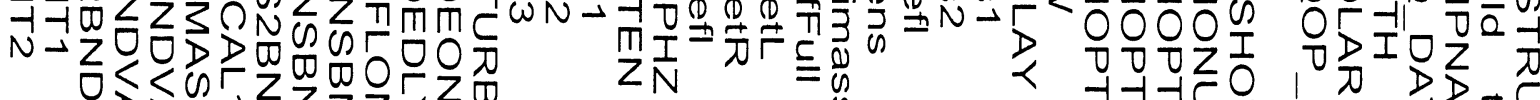

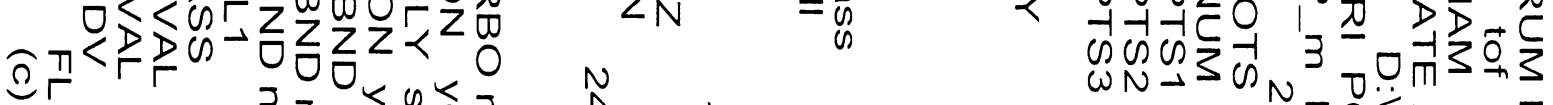

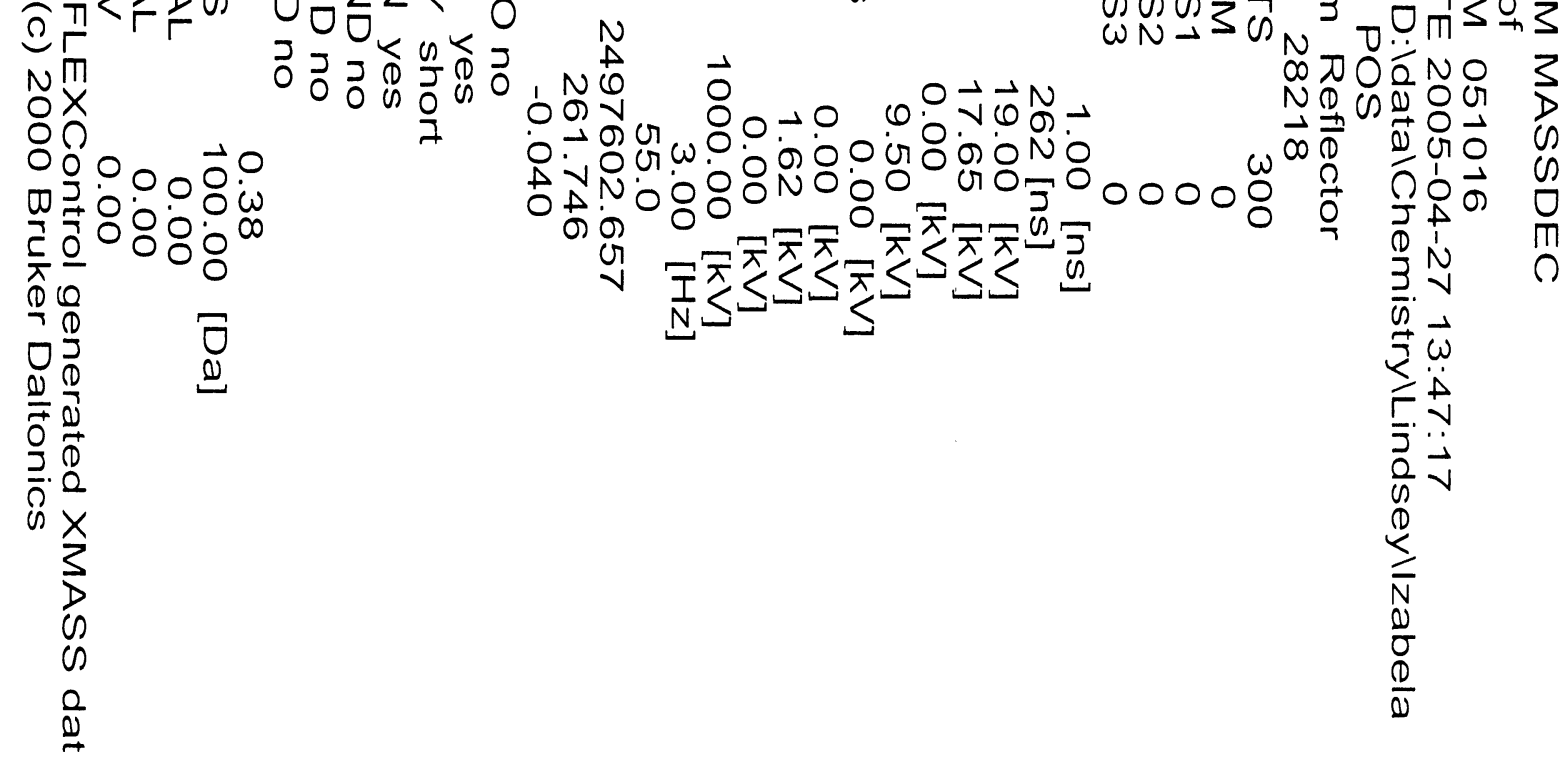




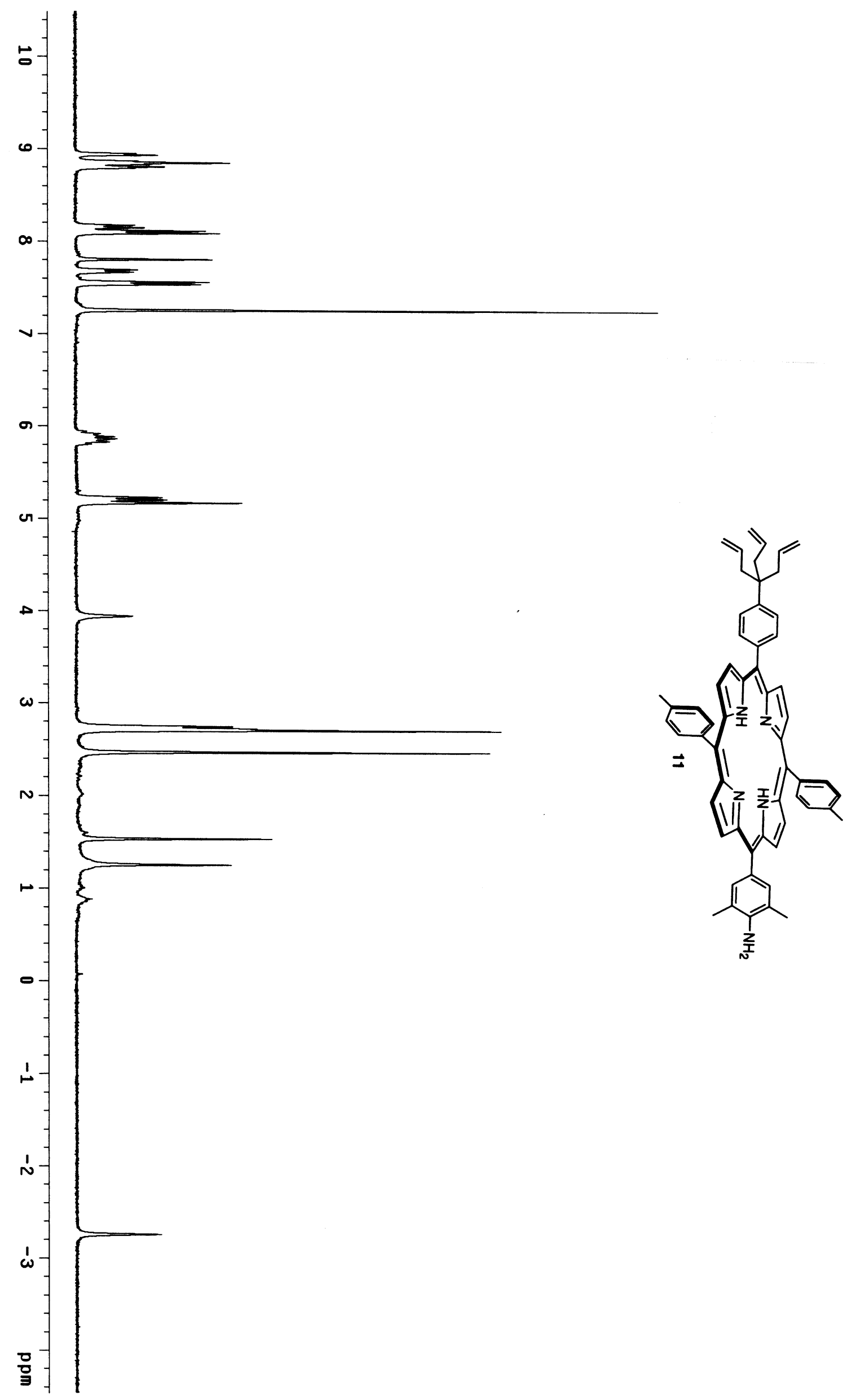




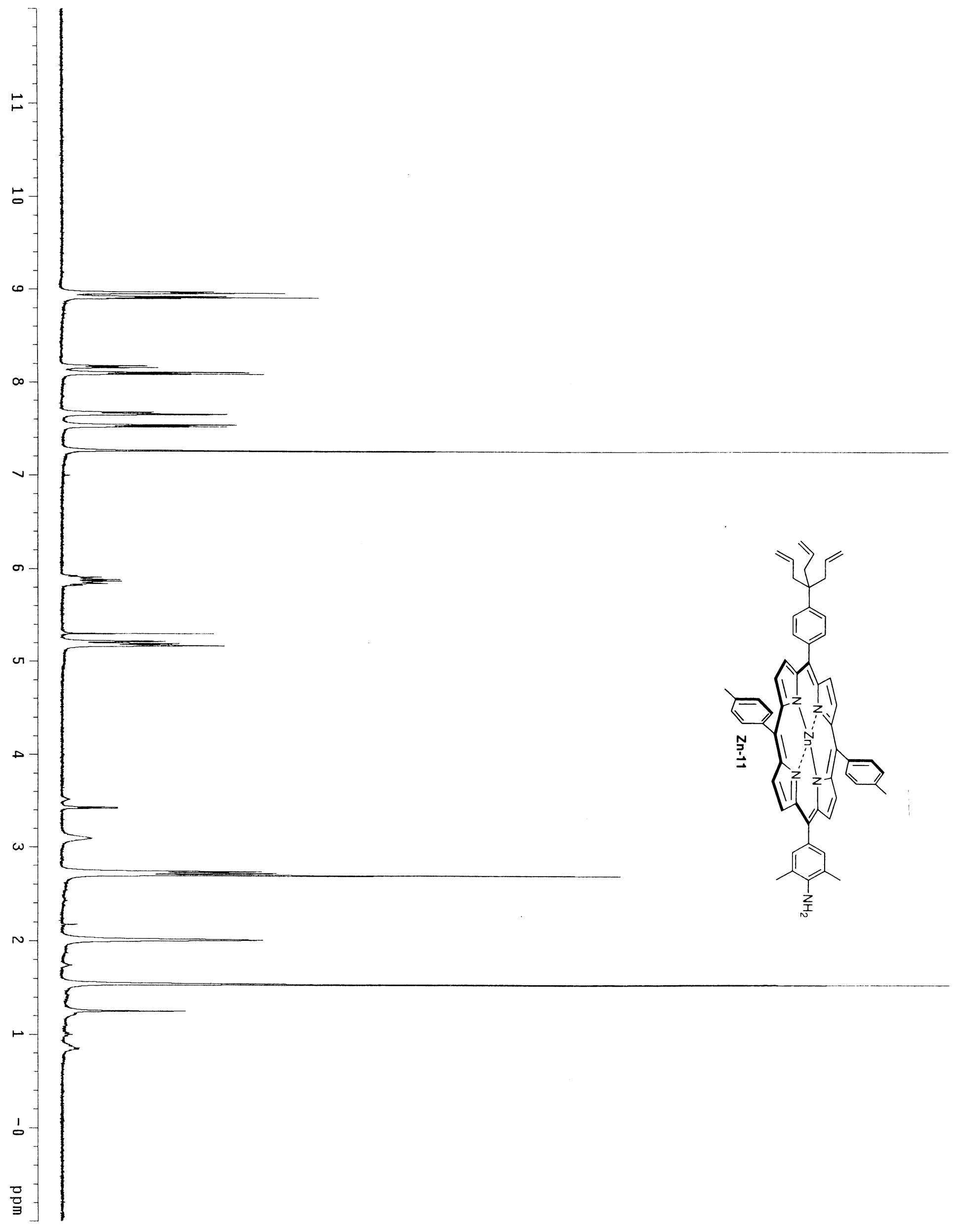




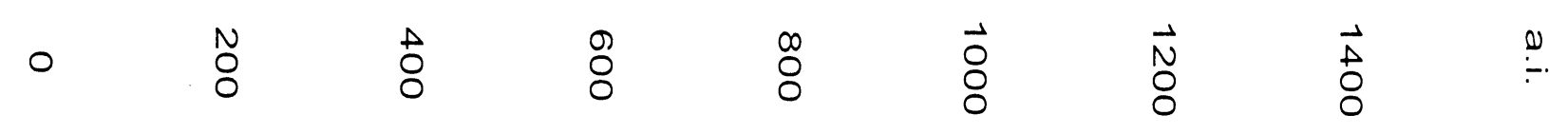

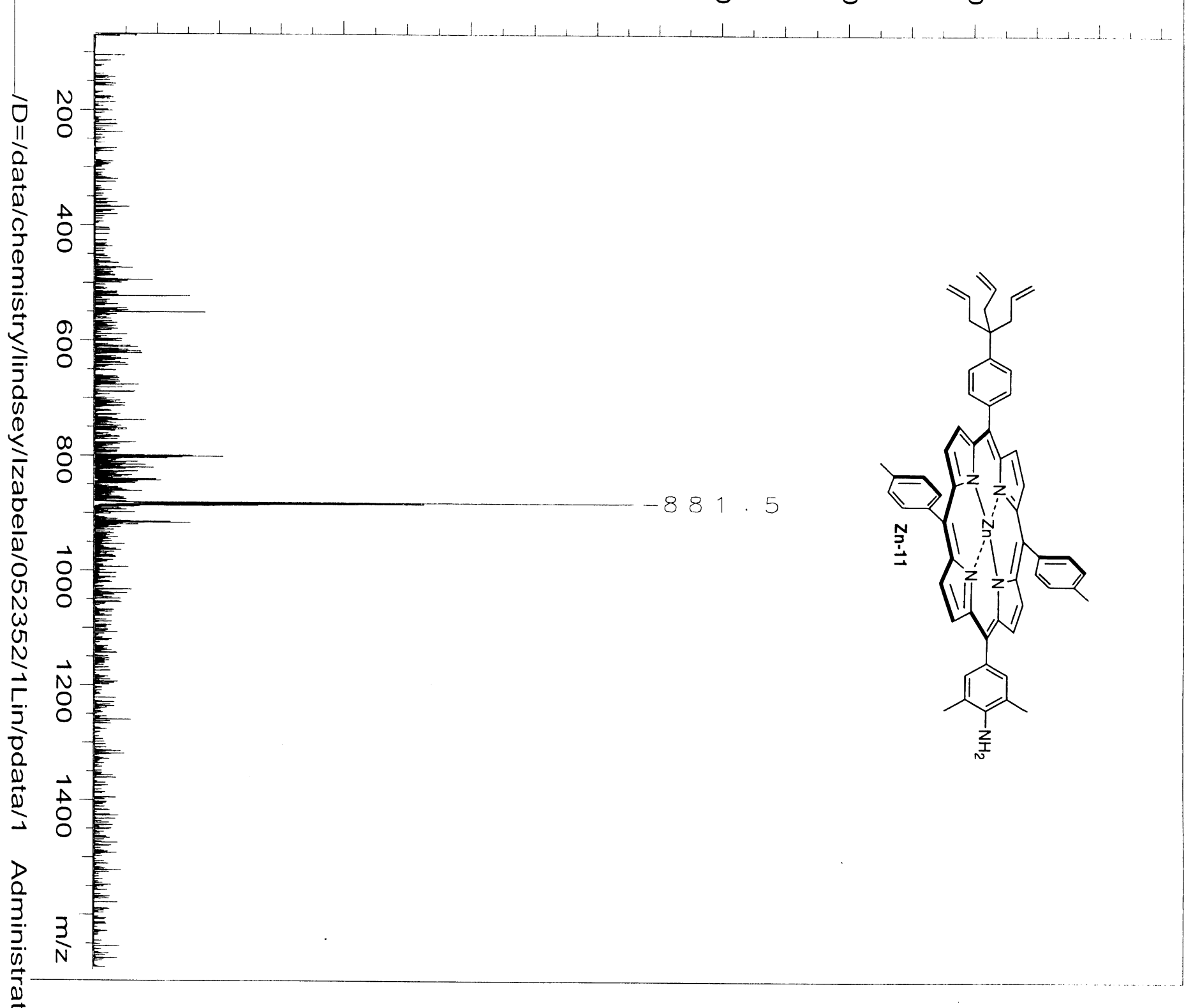

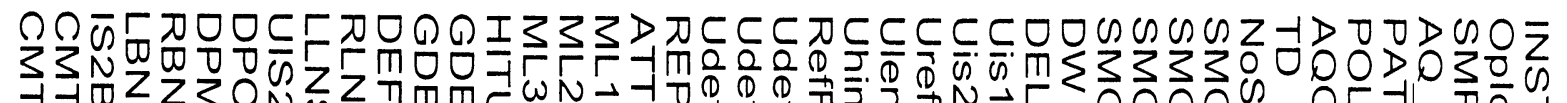

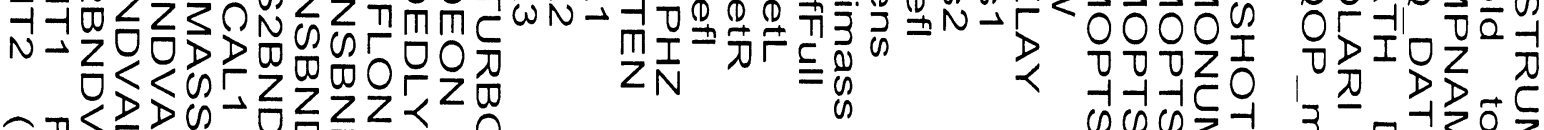

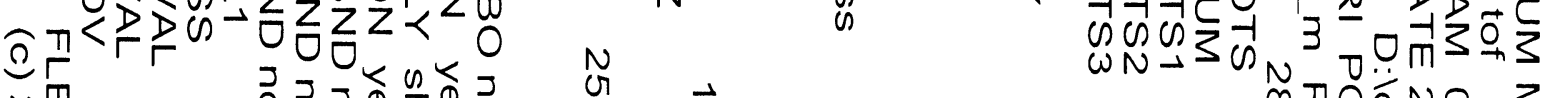

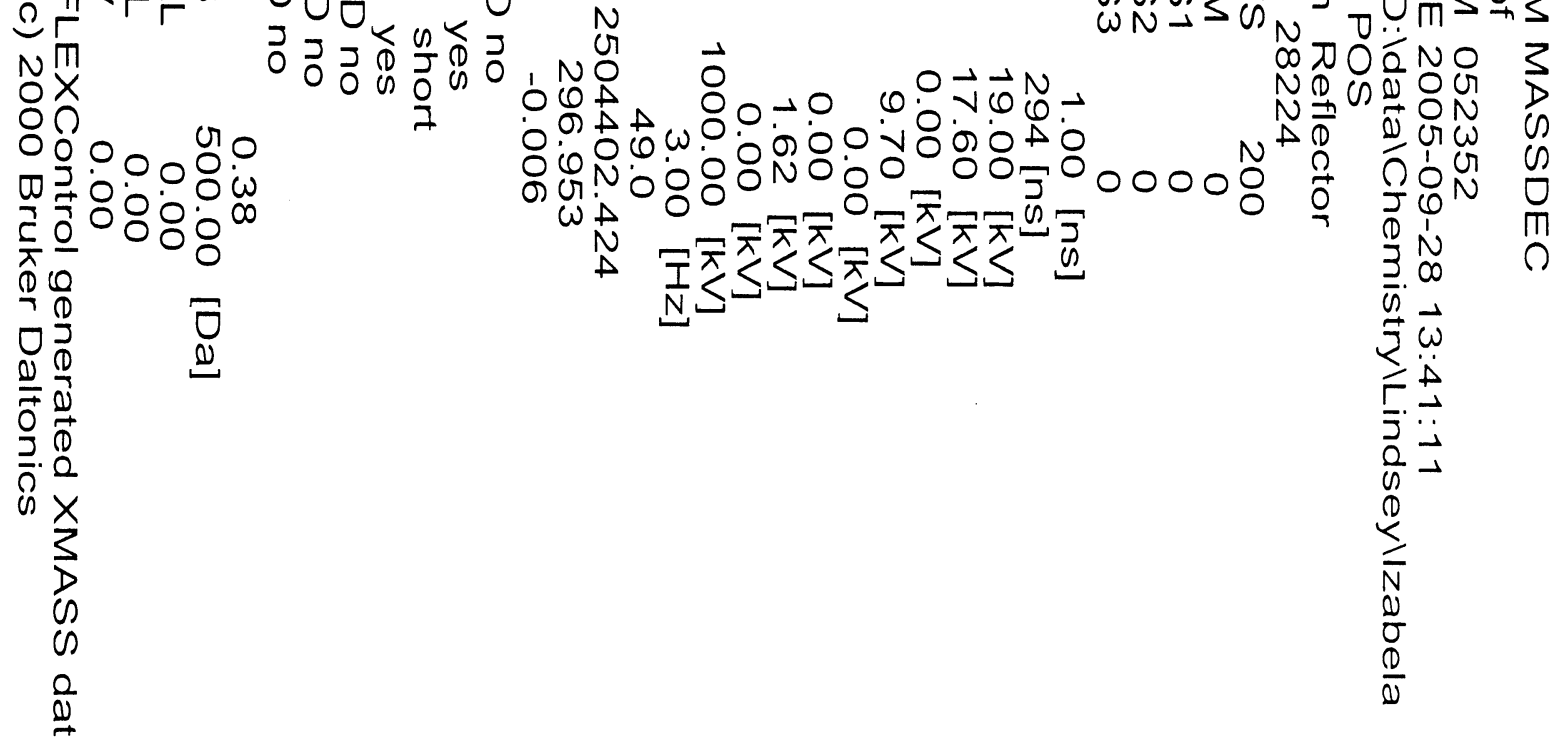




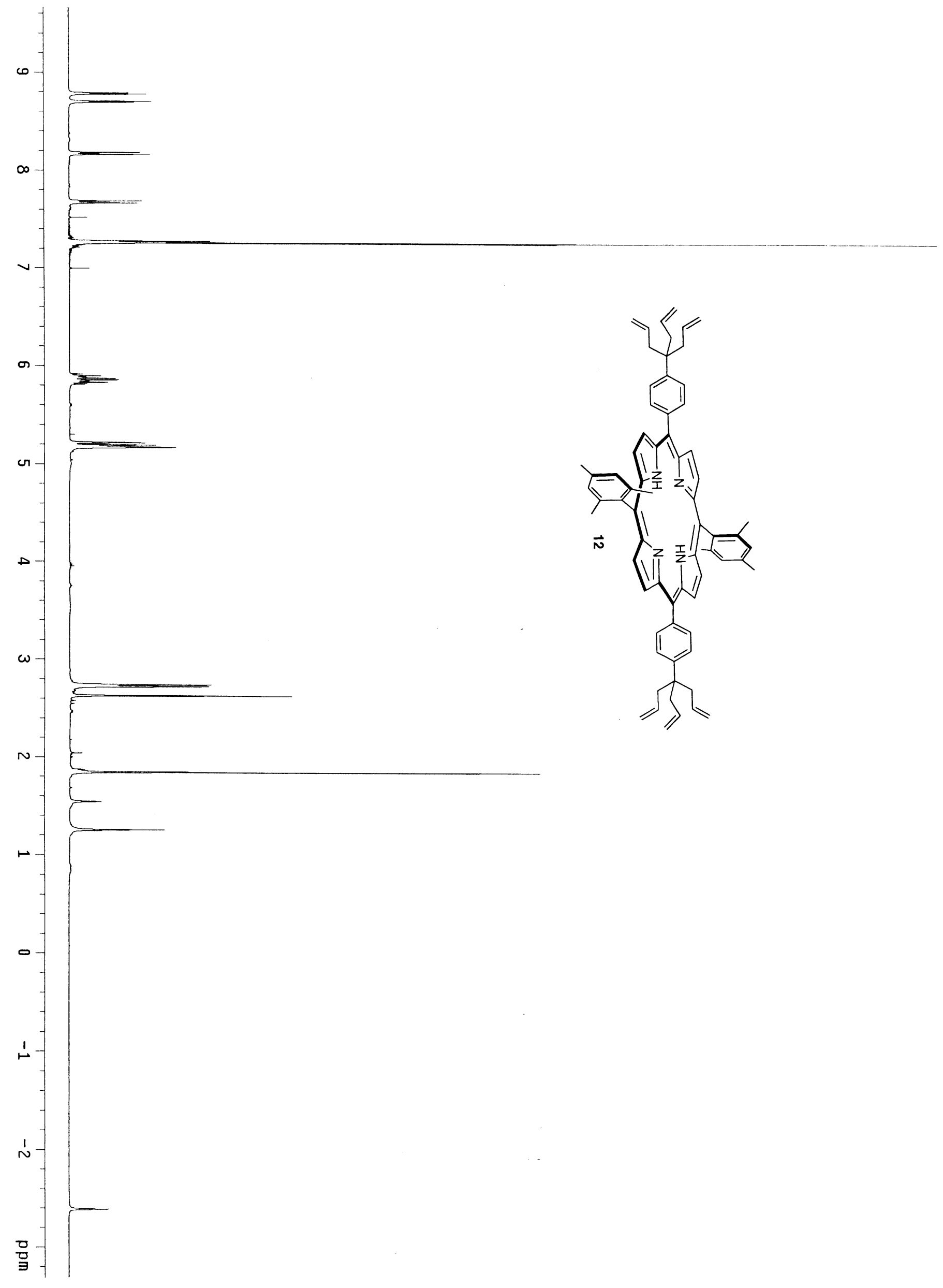




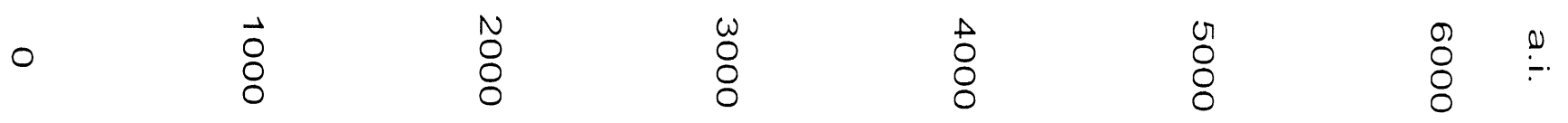

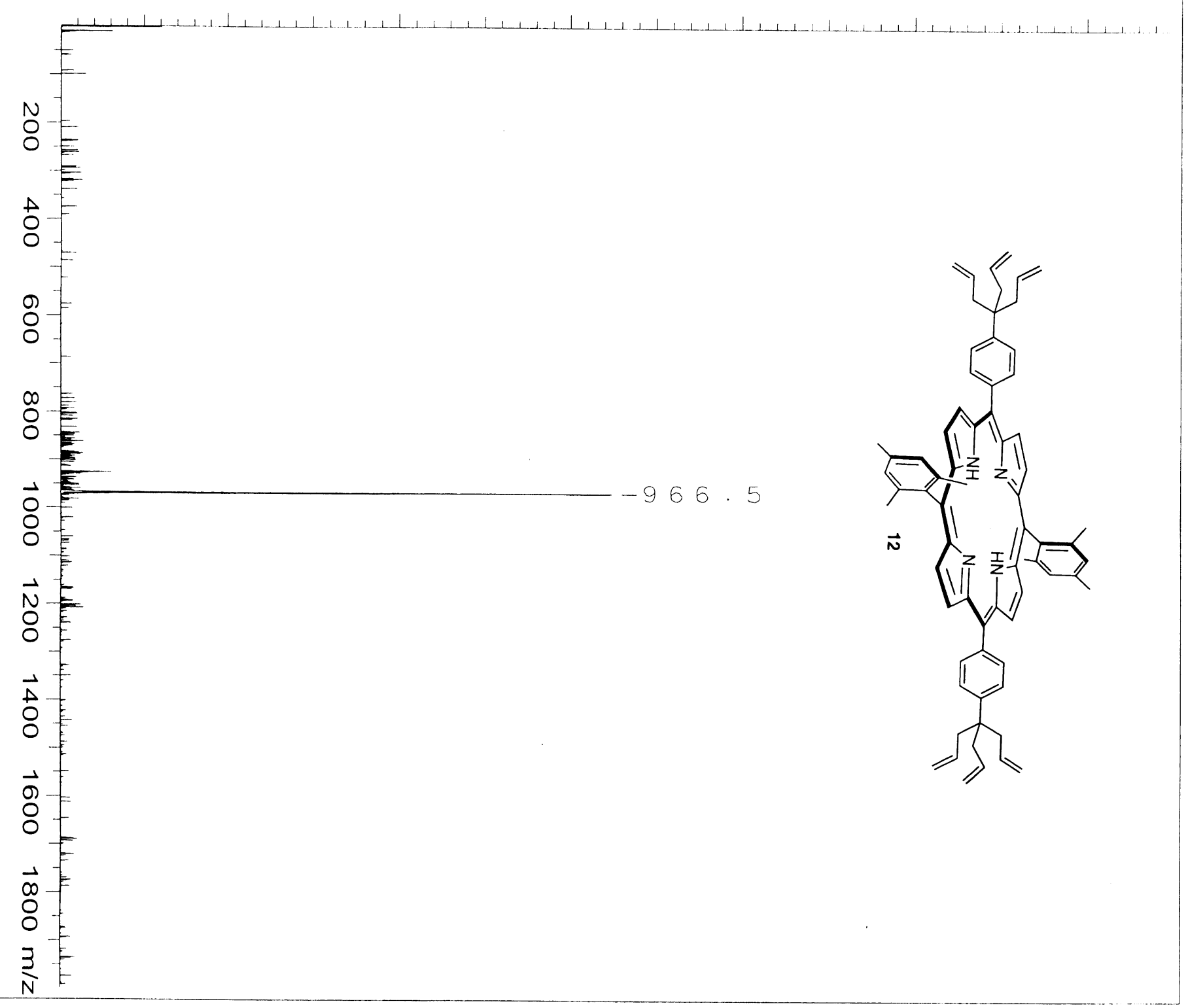

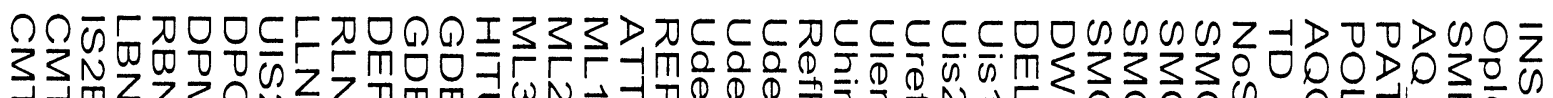

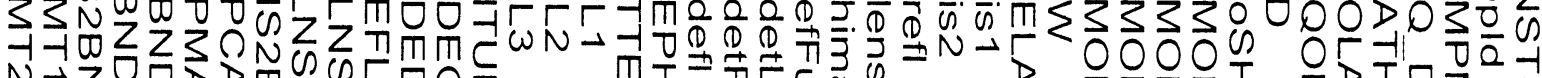

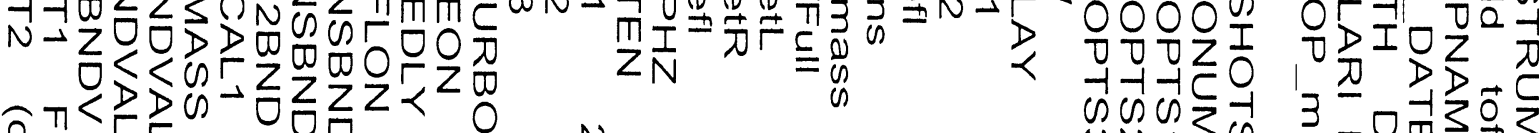

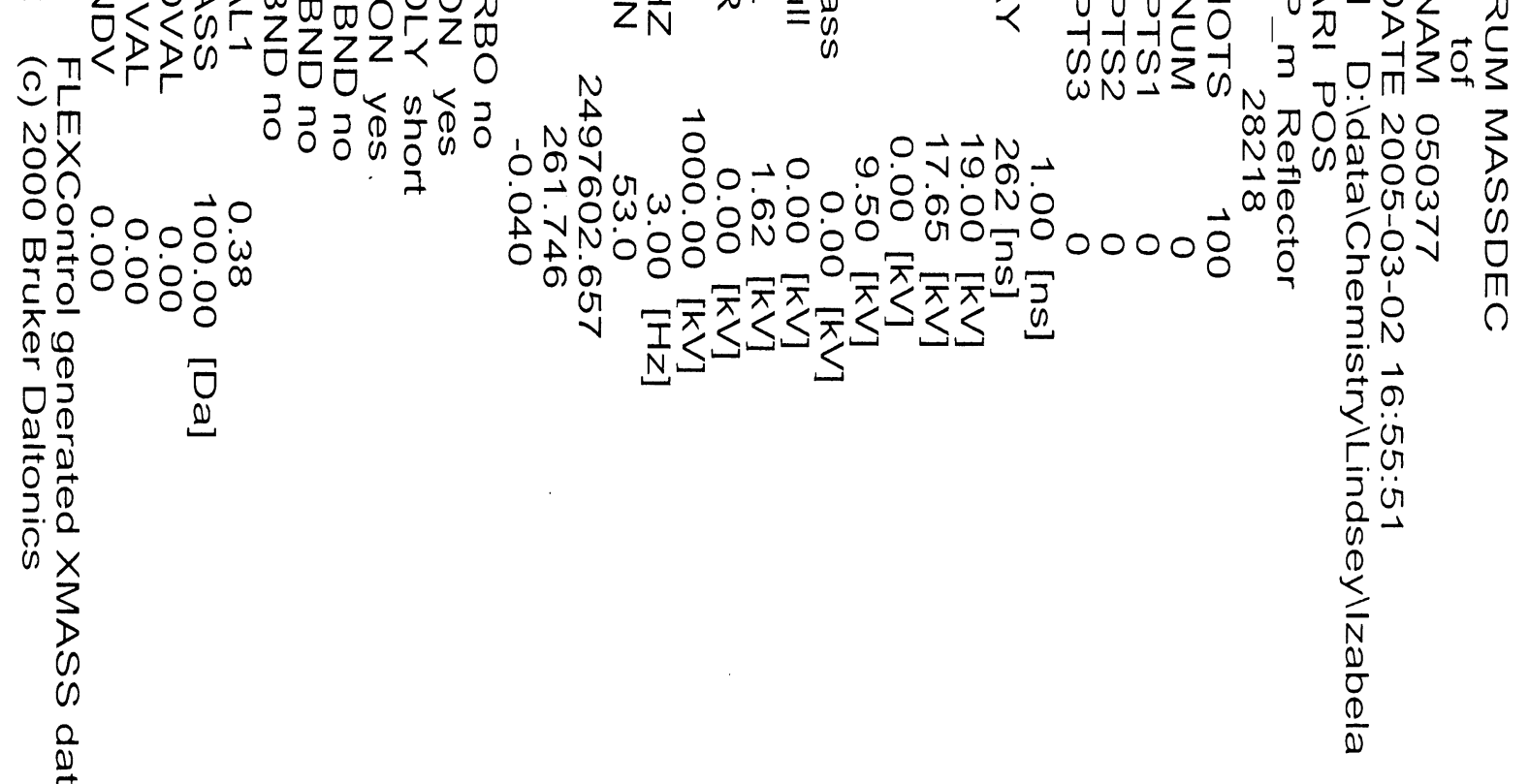



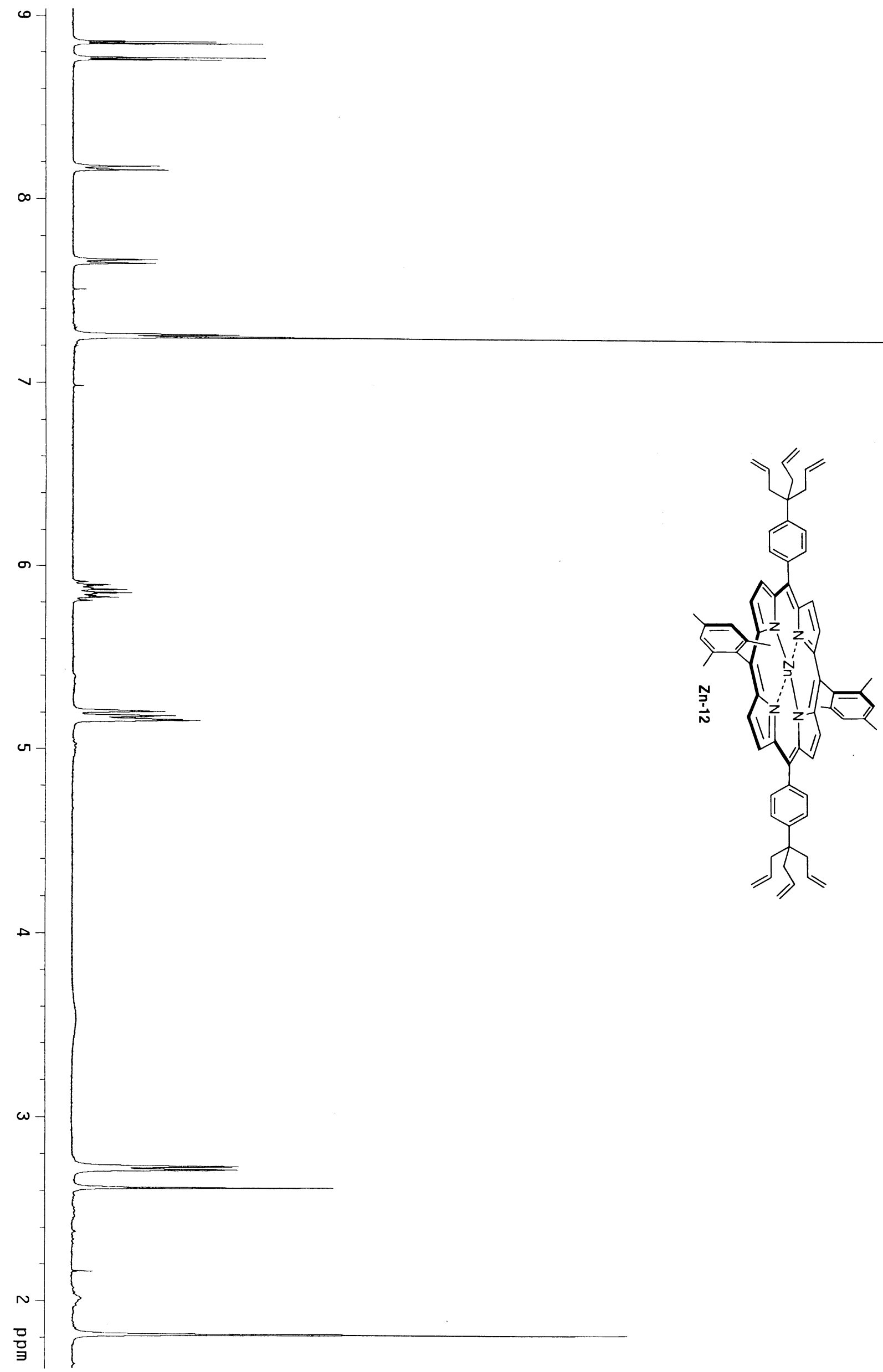


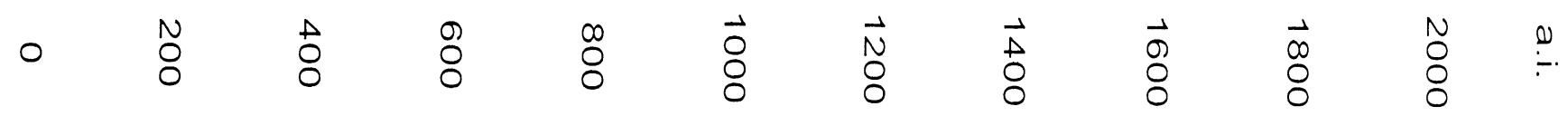

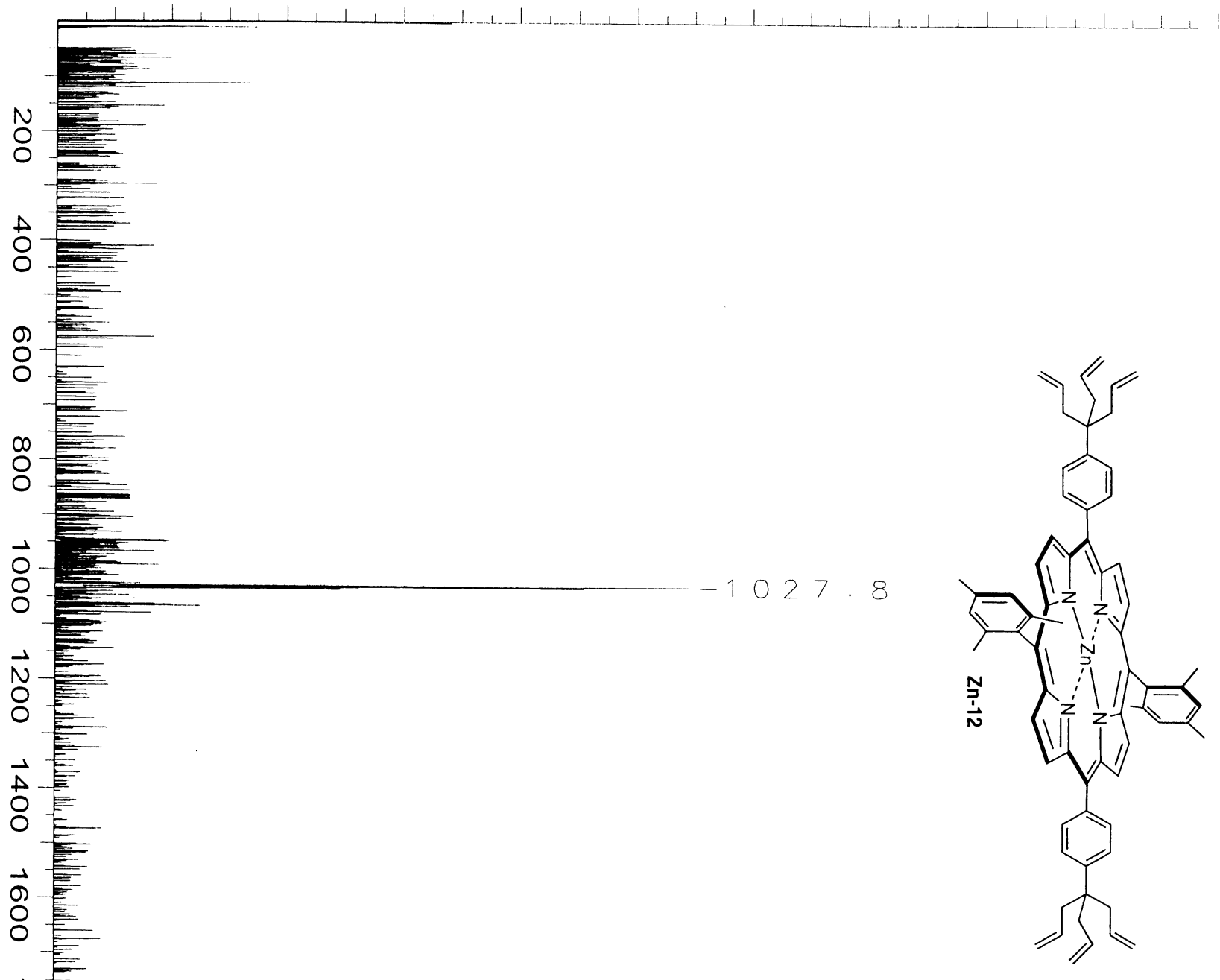

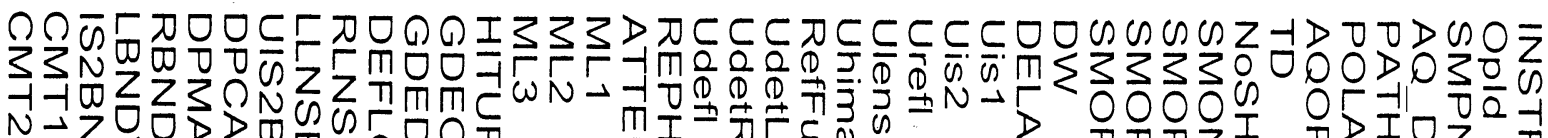

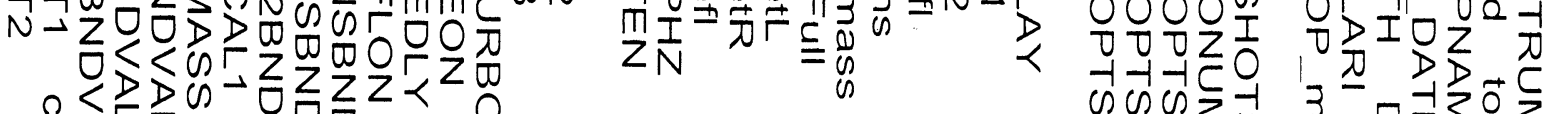
$3<\unrhd \unrhd \omega-0 Z Z Z<Z_{0}$

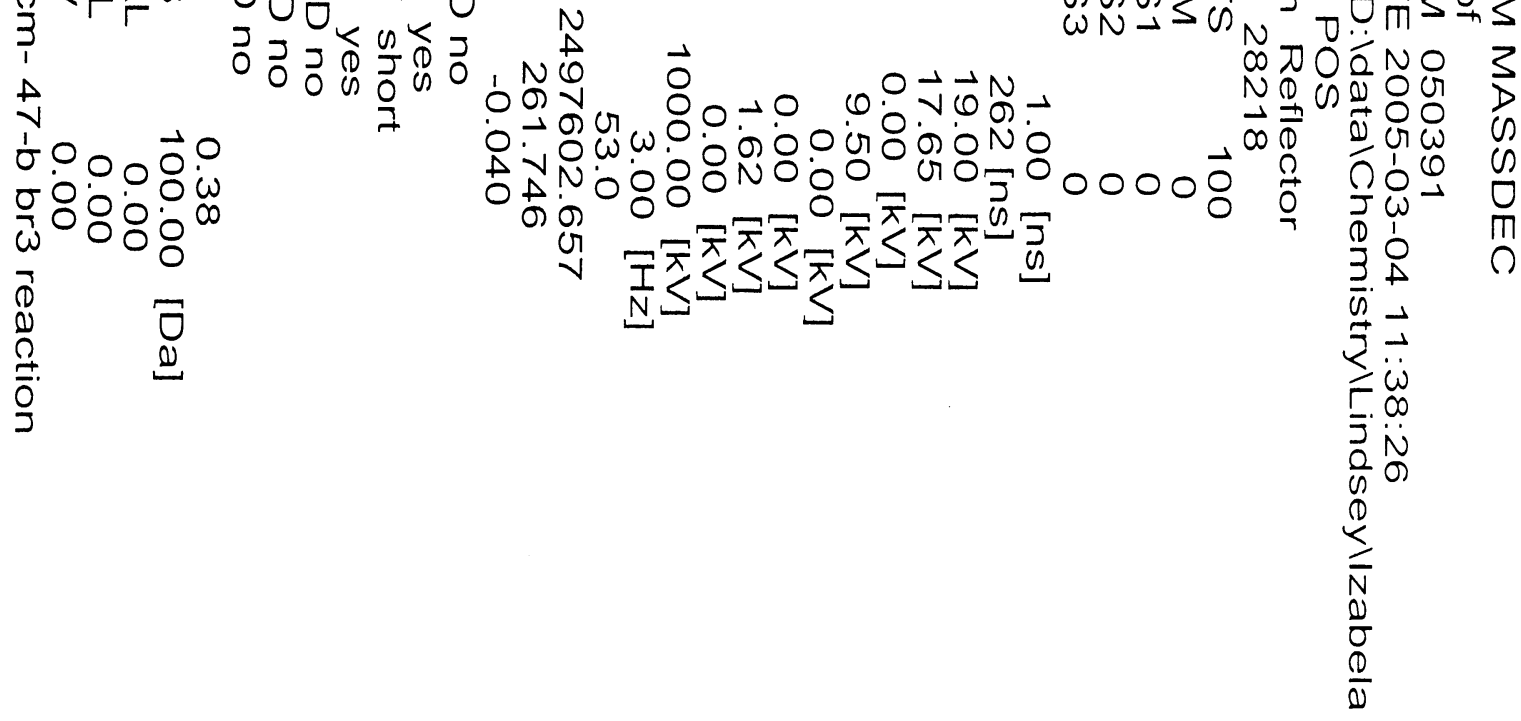



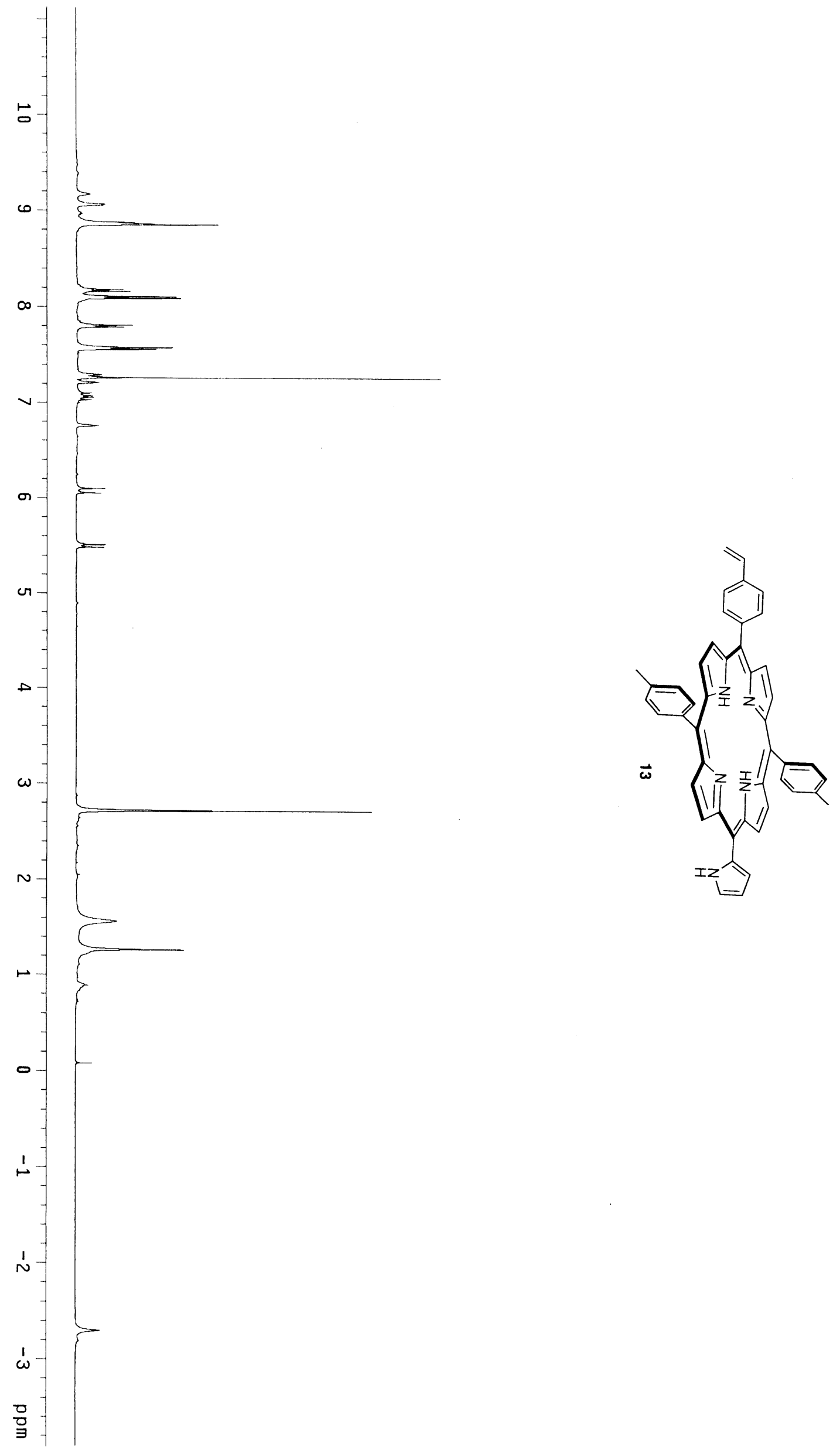

0
ठ
ัั
๕
。
芒

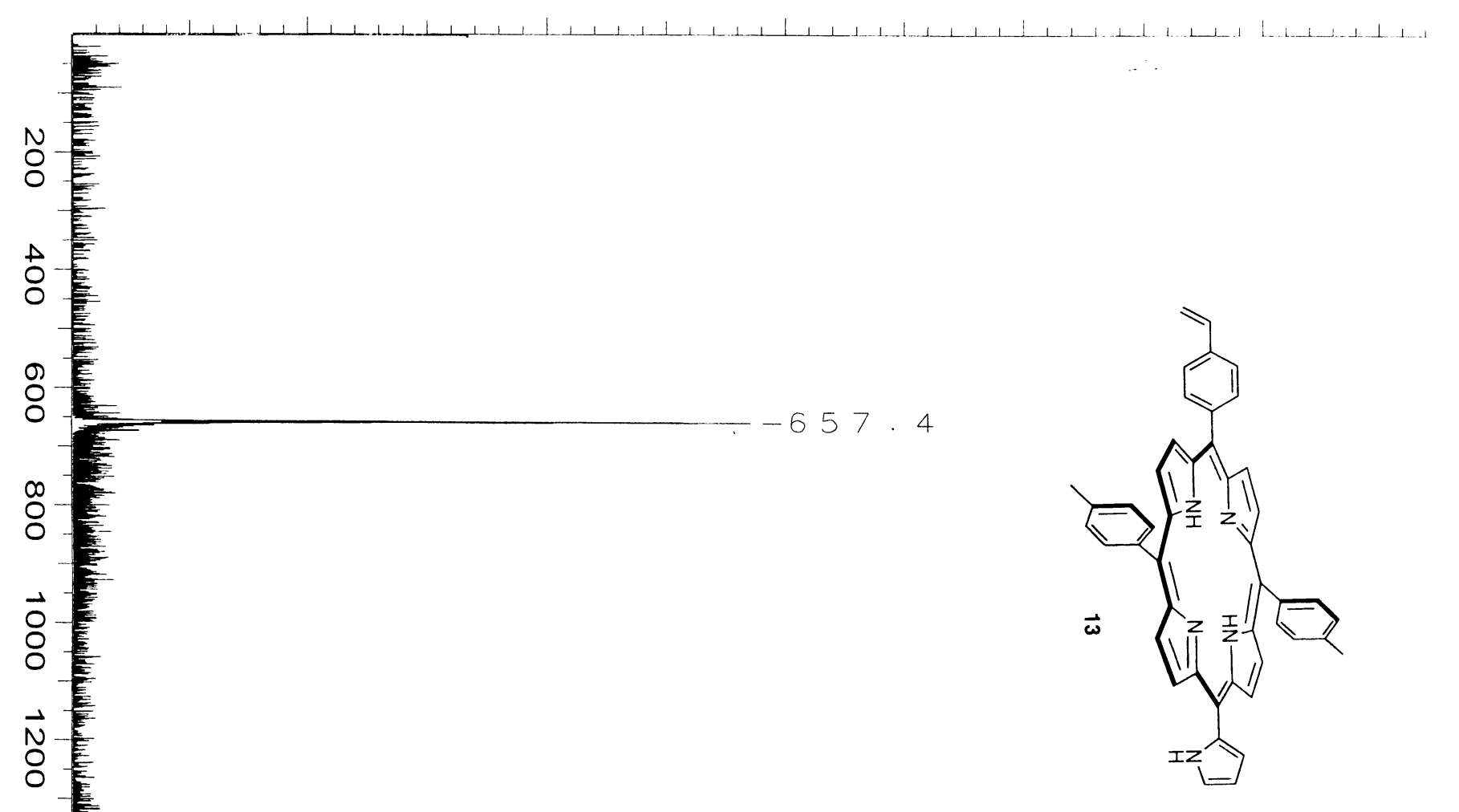

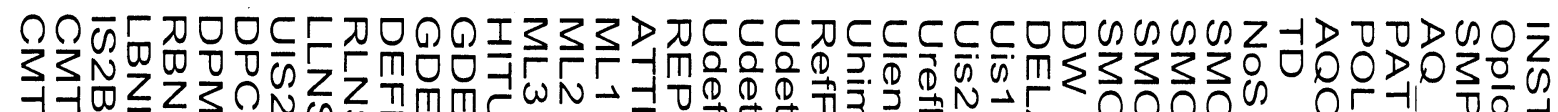

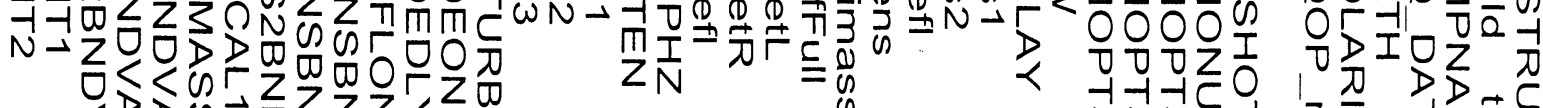

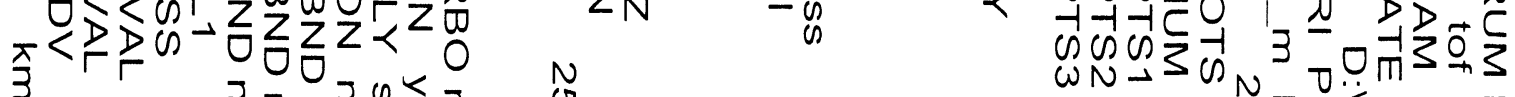

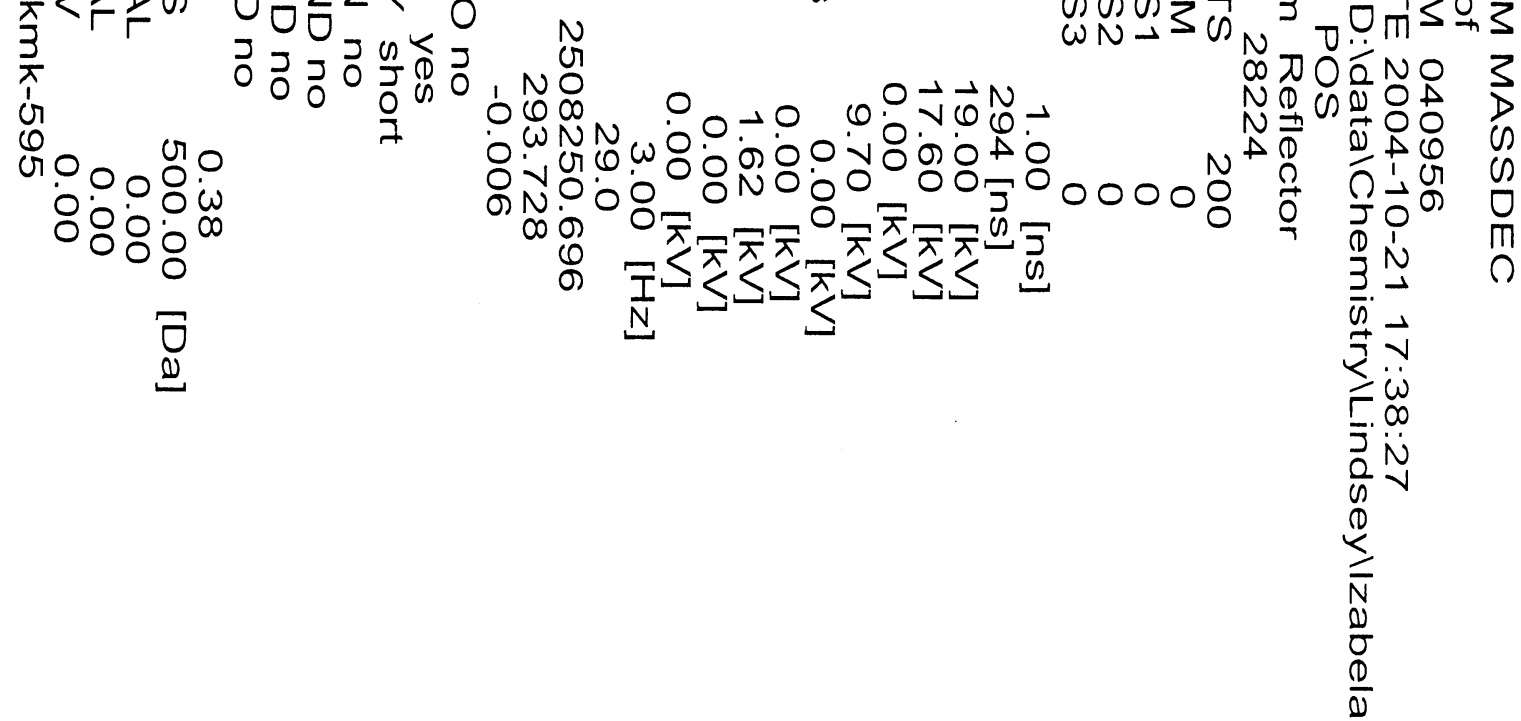



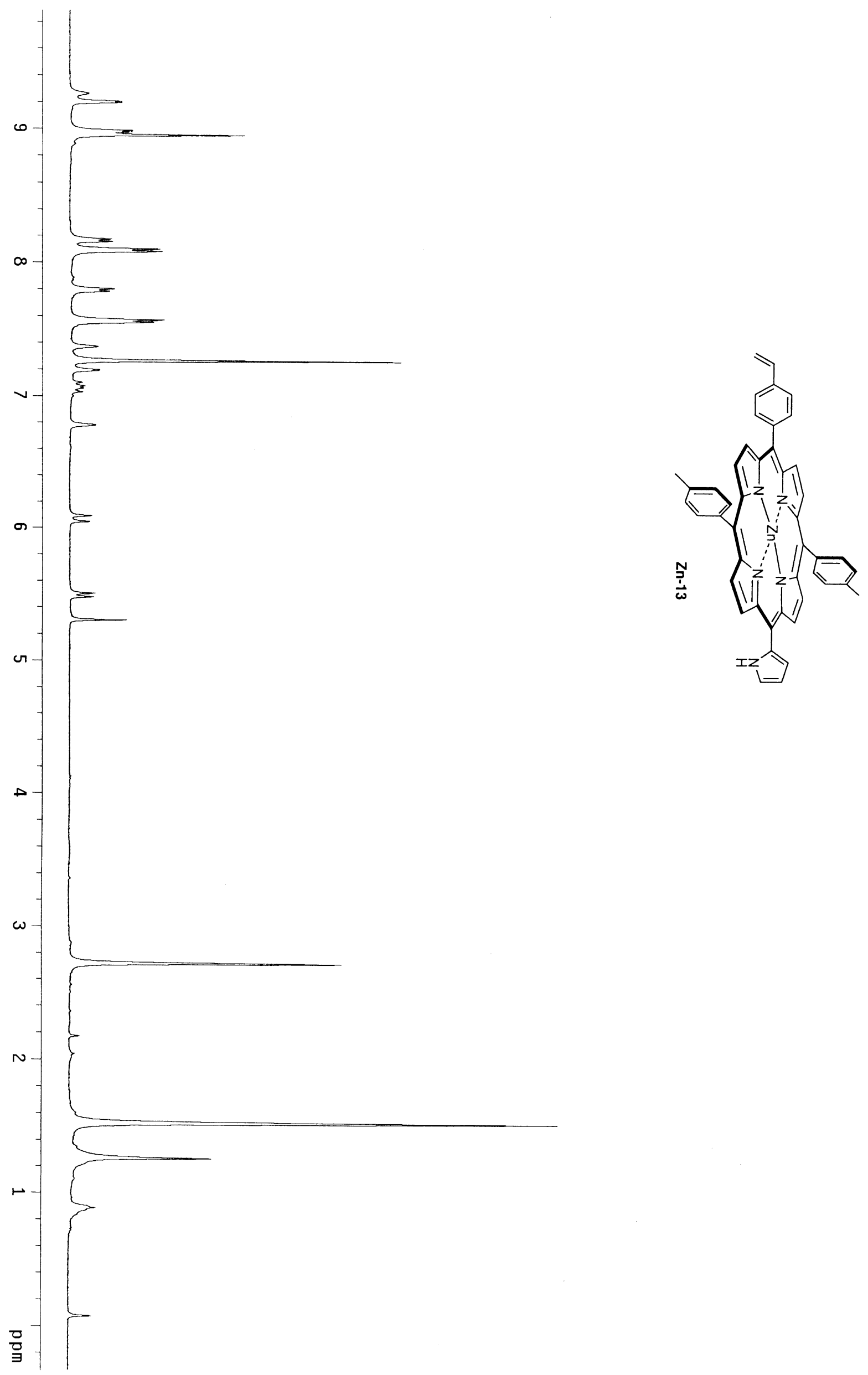

。
$\stackrel{\square}{8}$
흠
喜
:
高

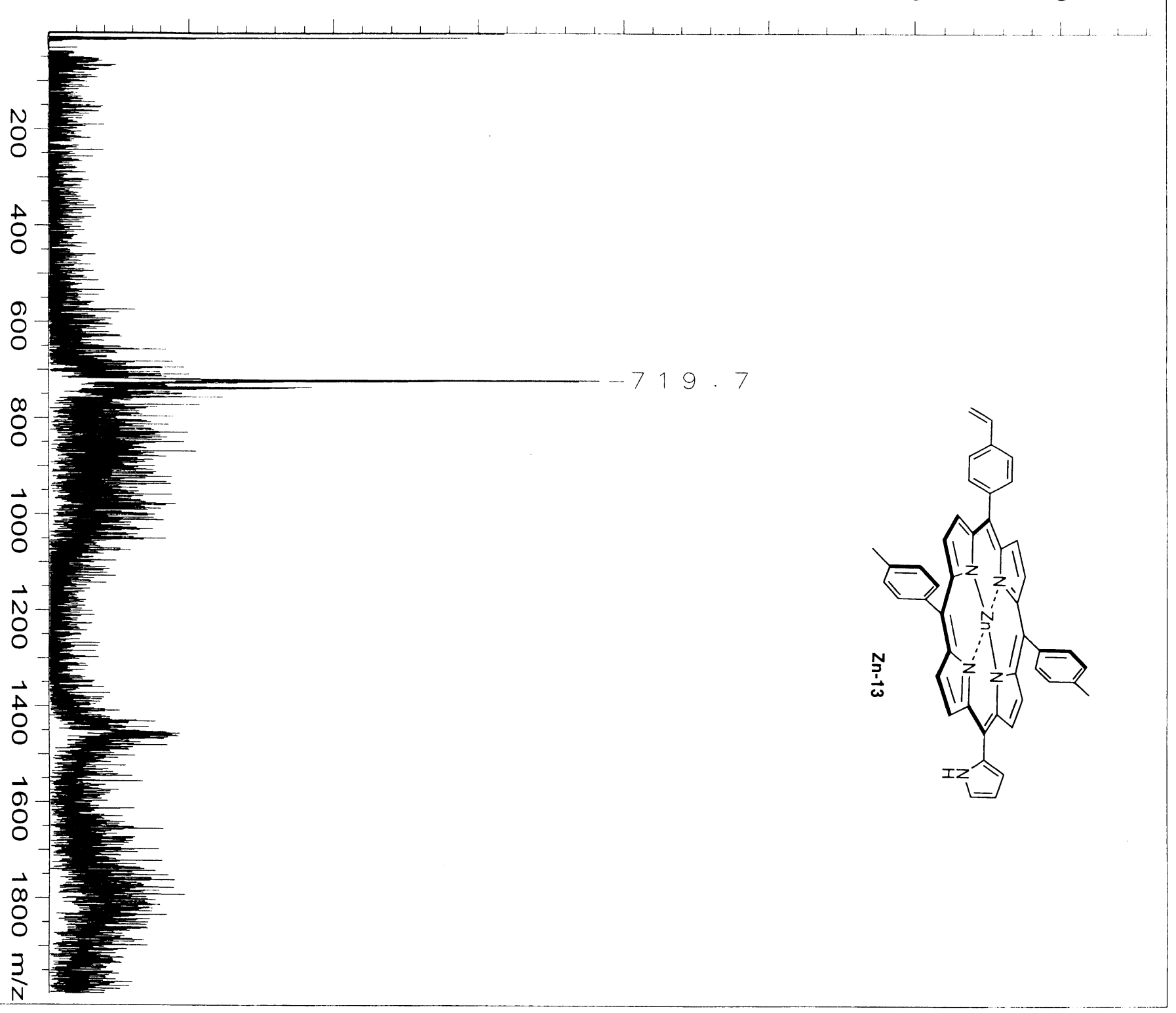

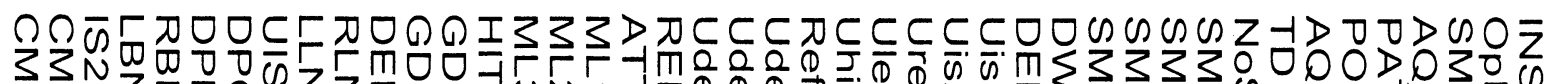

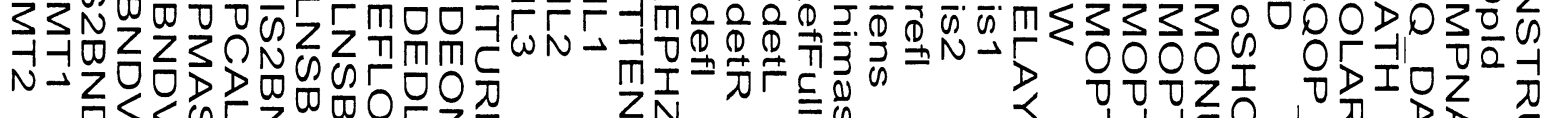

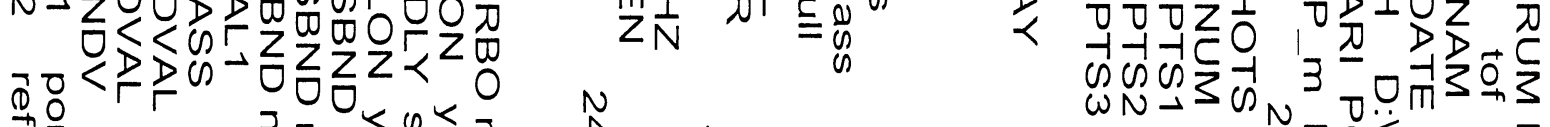

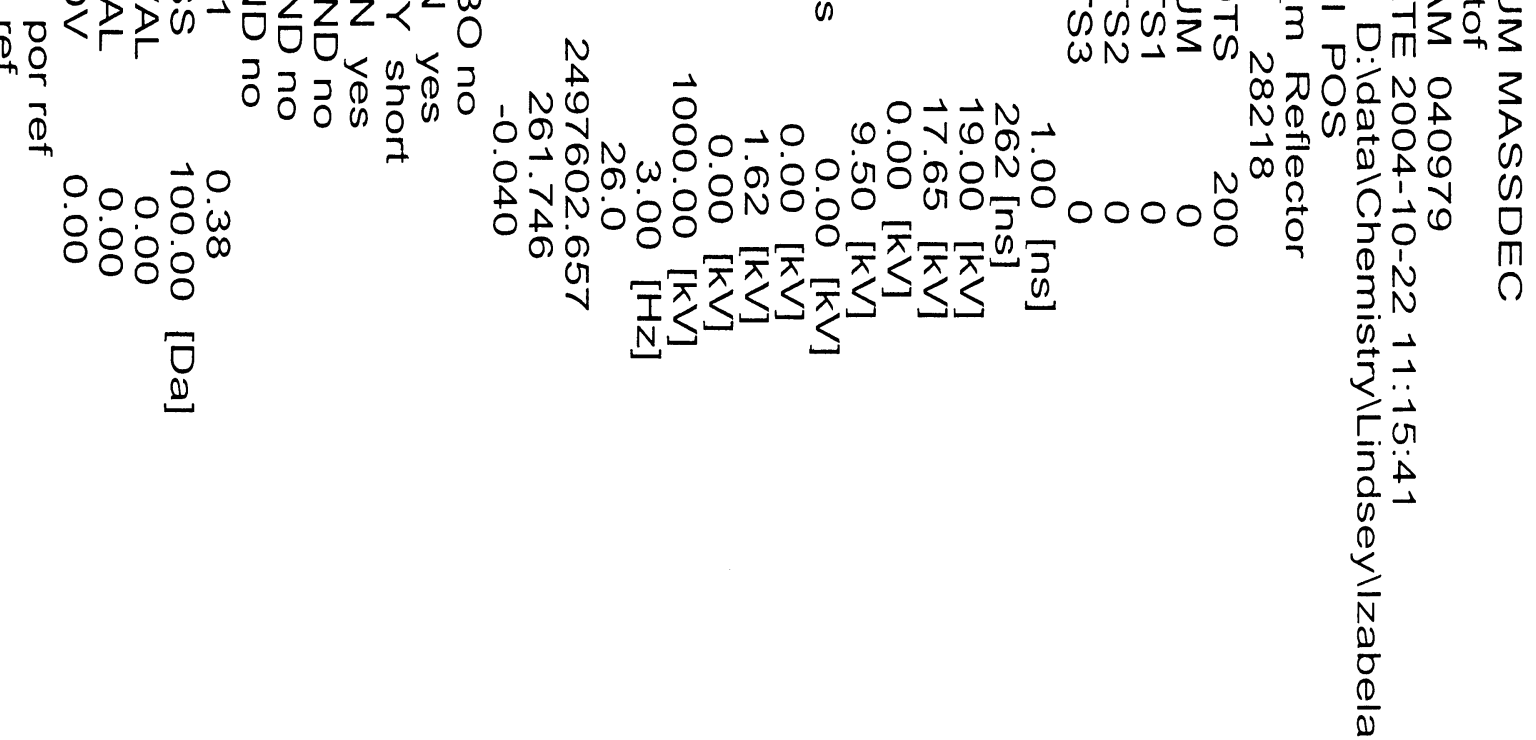




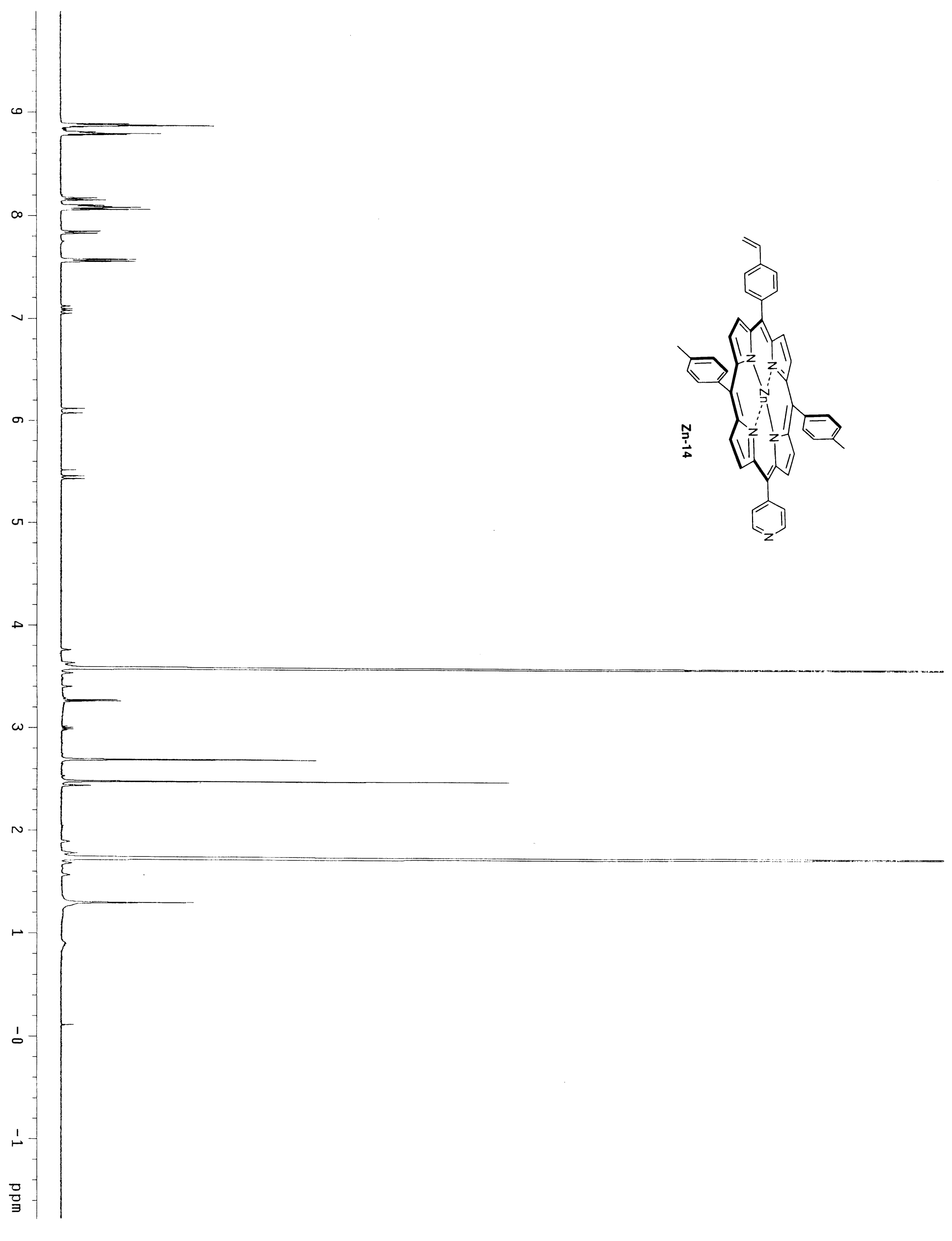




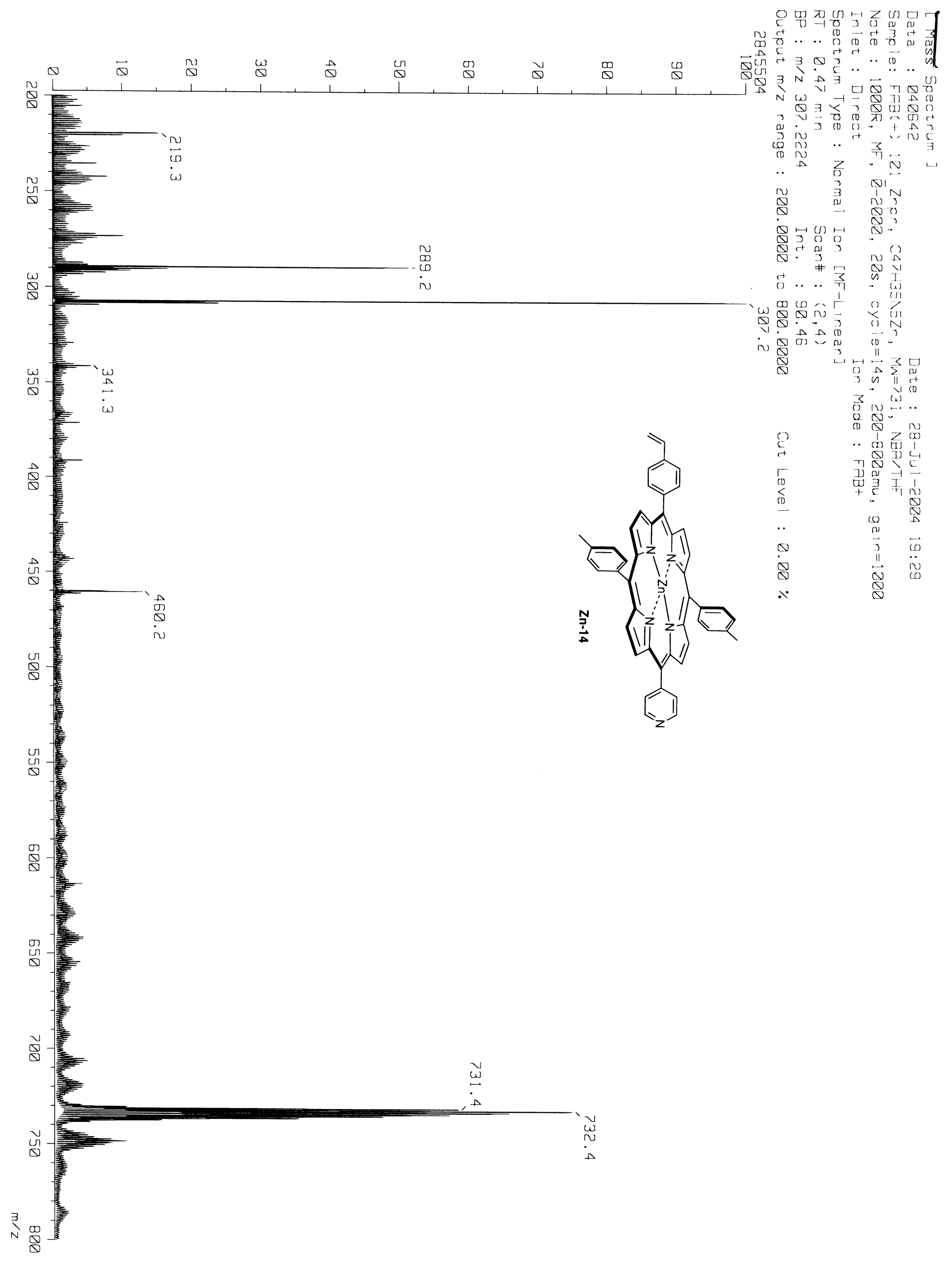




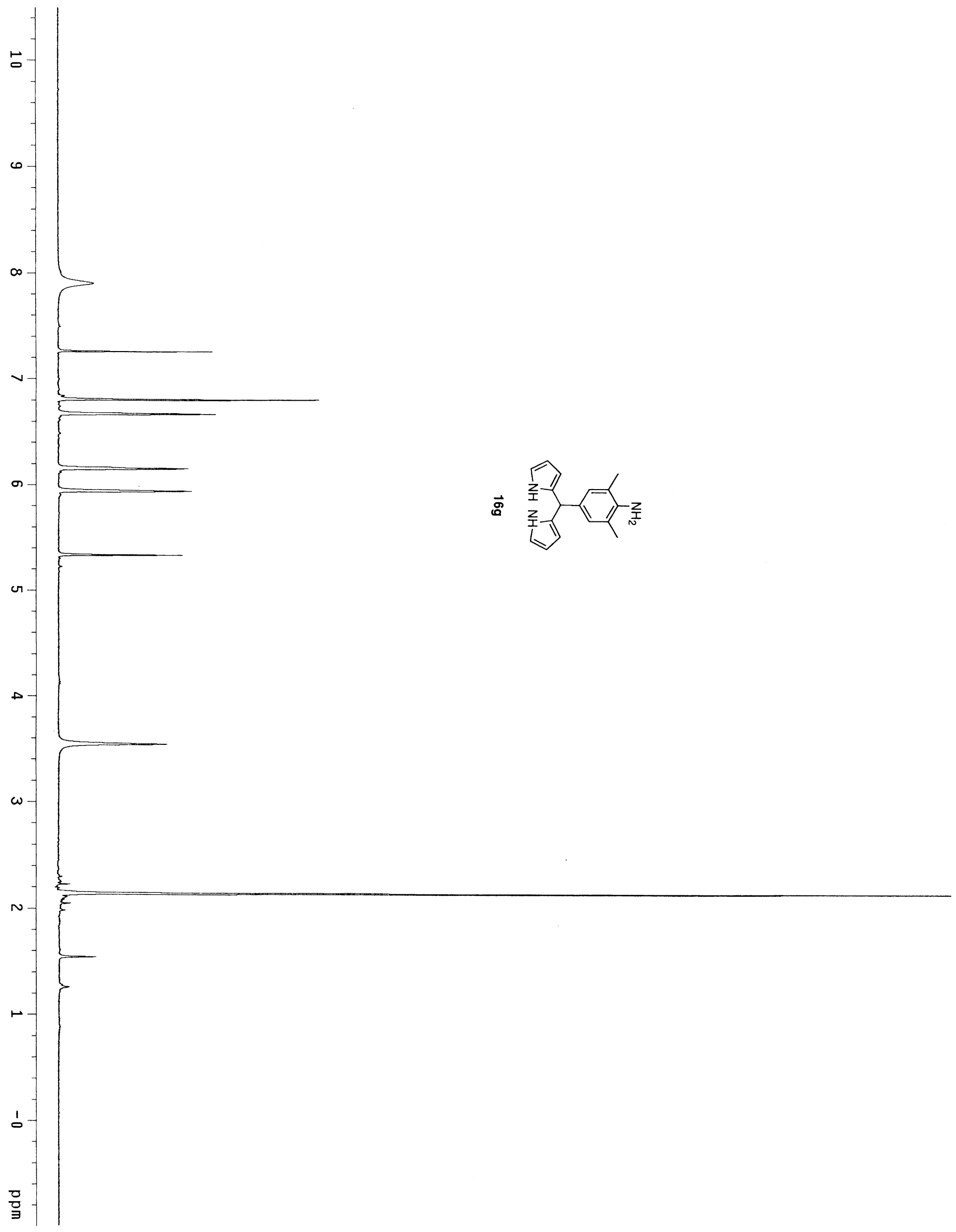



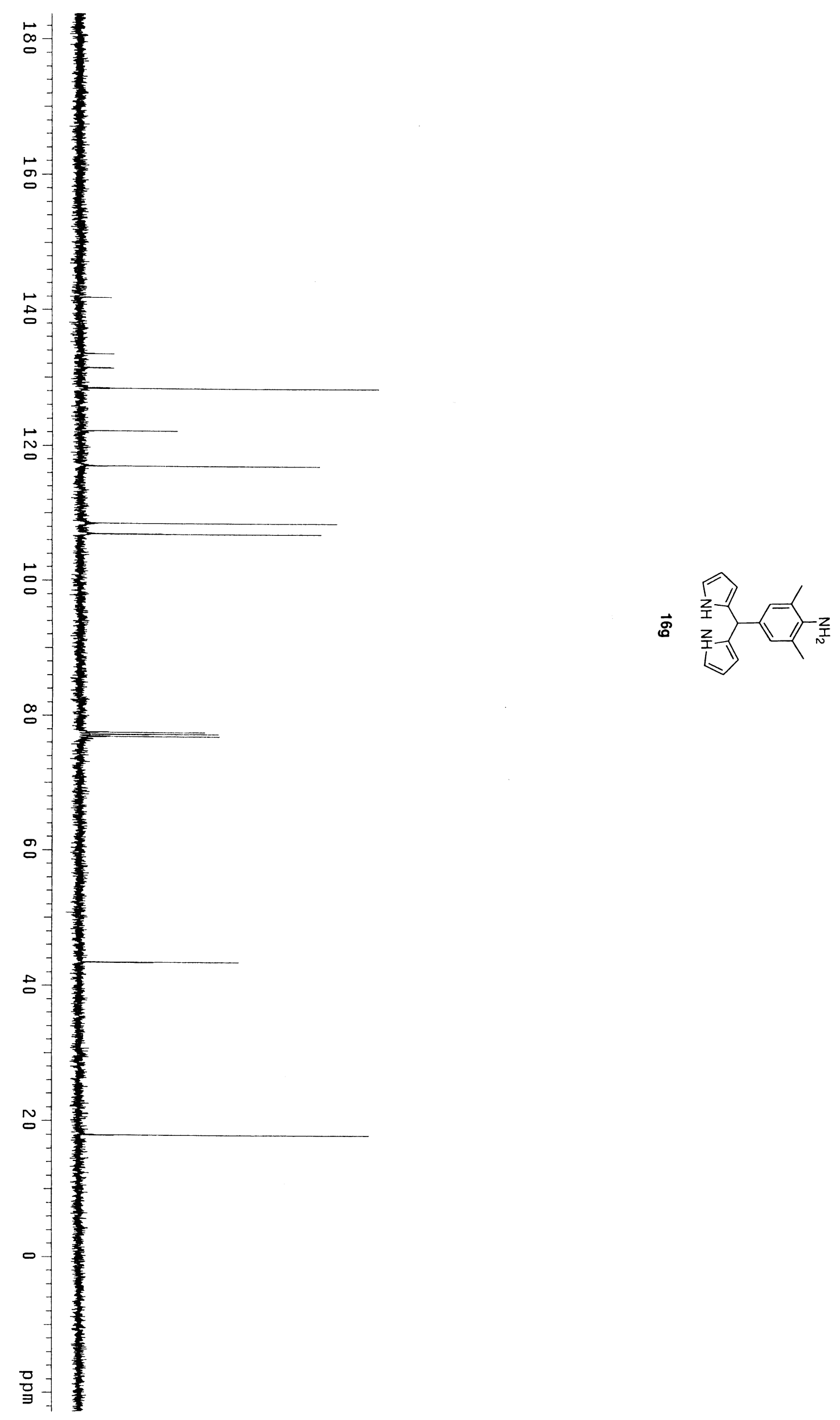


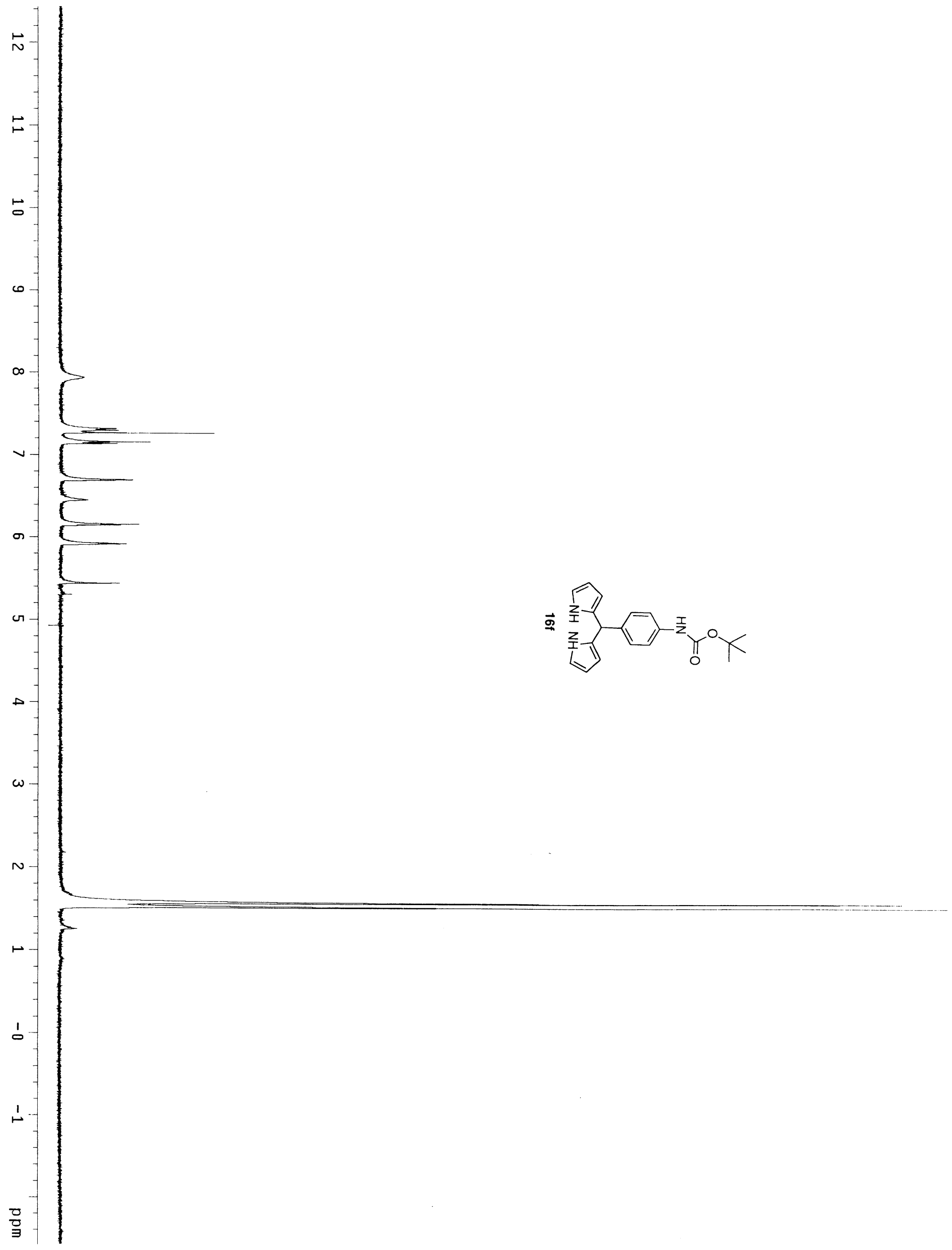




$$
\mid
$$




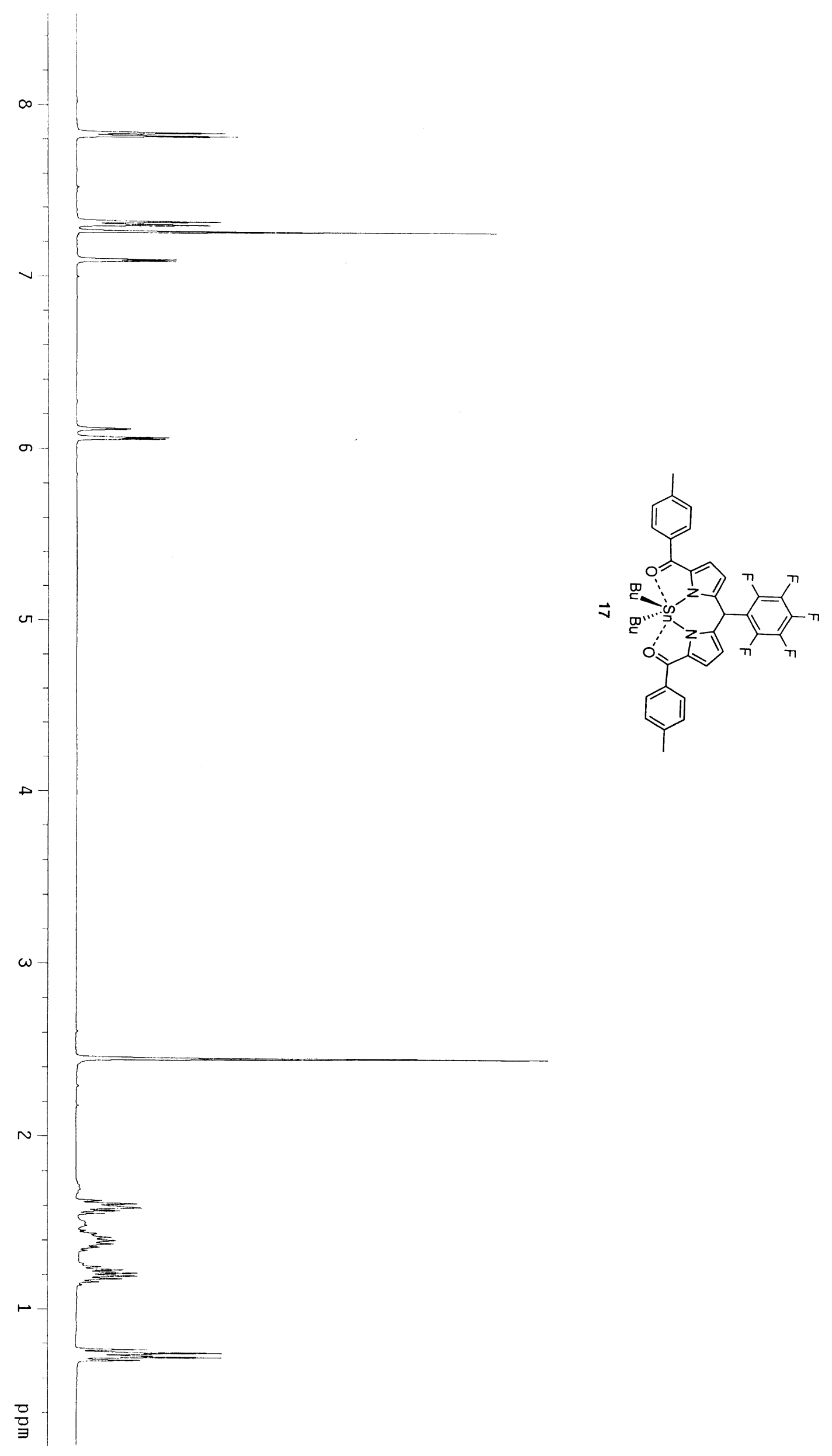




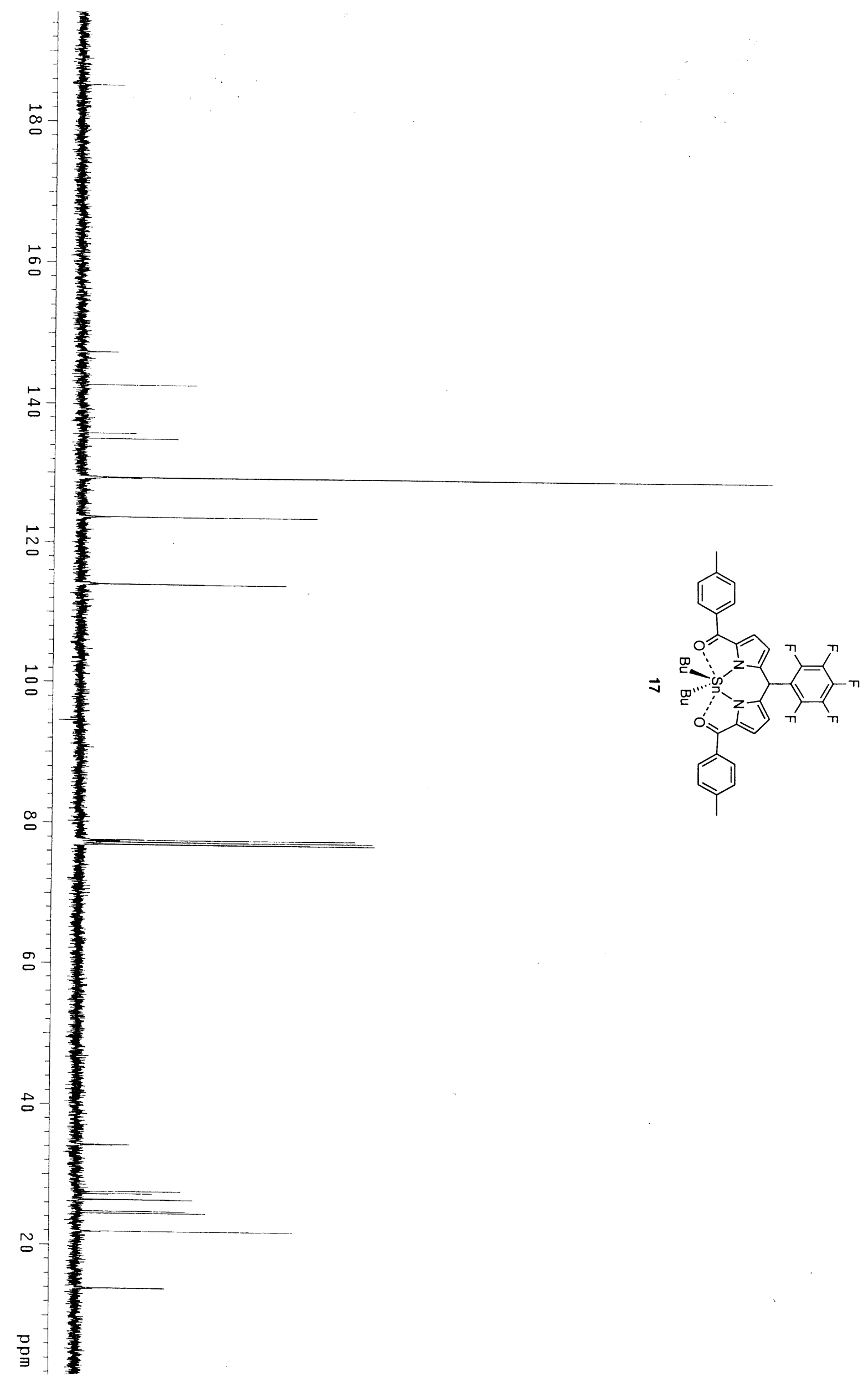



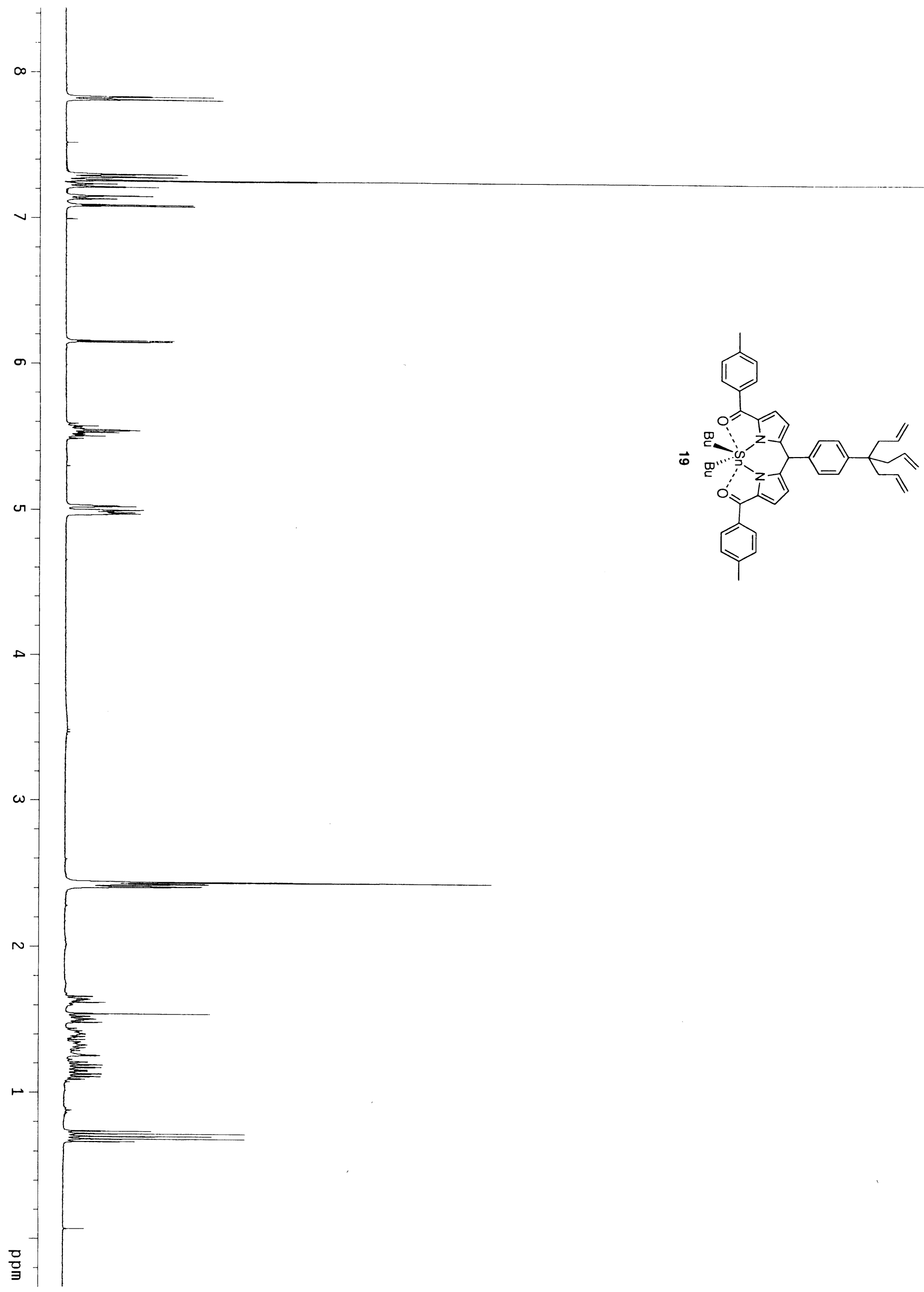


$$
E
$$




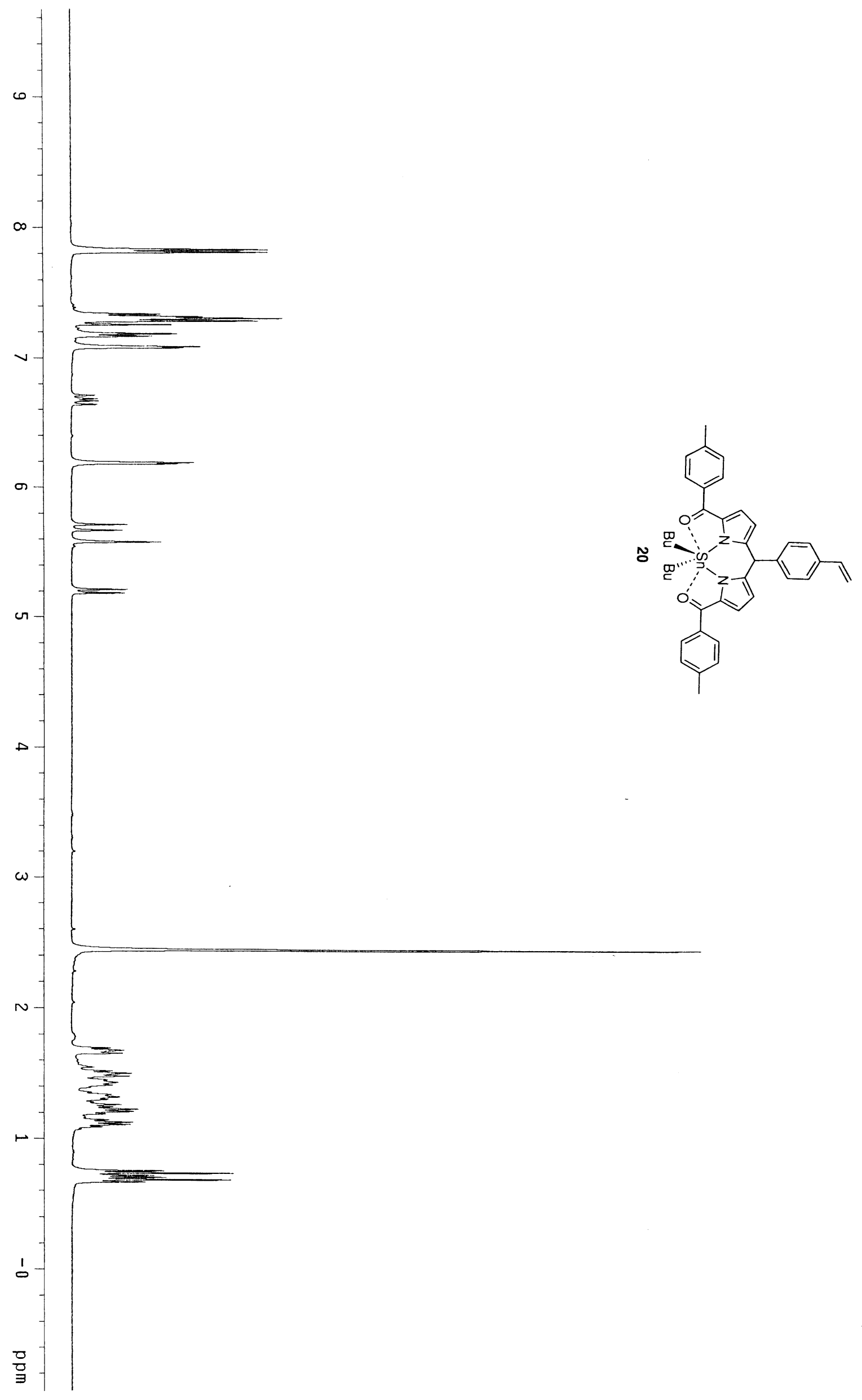




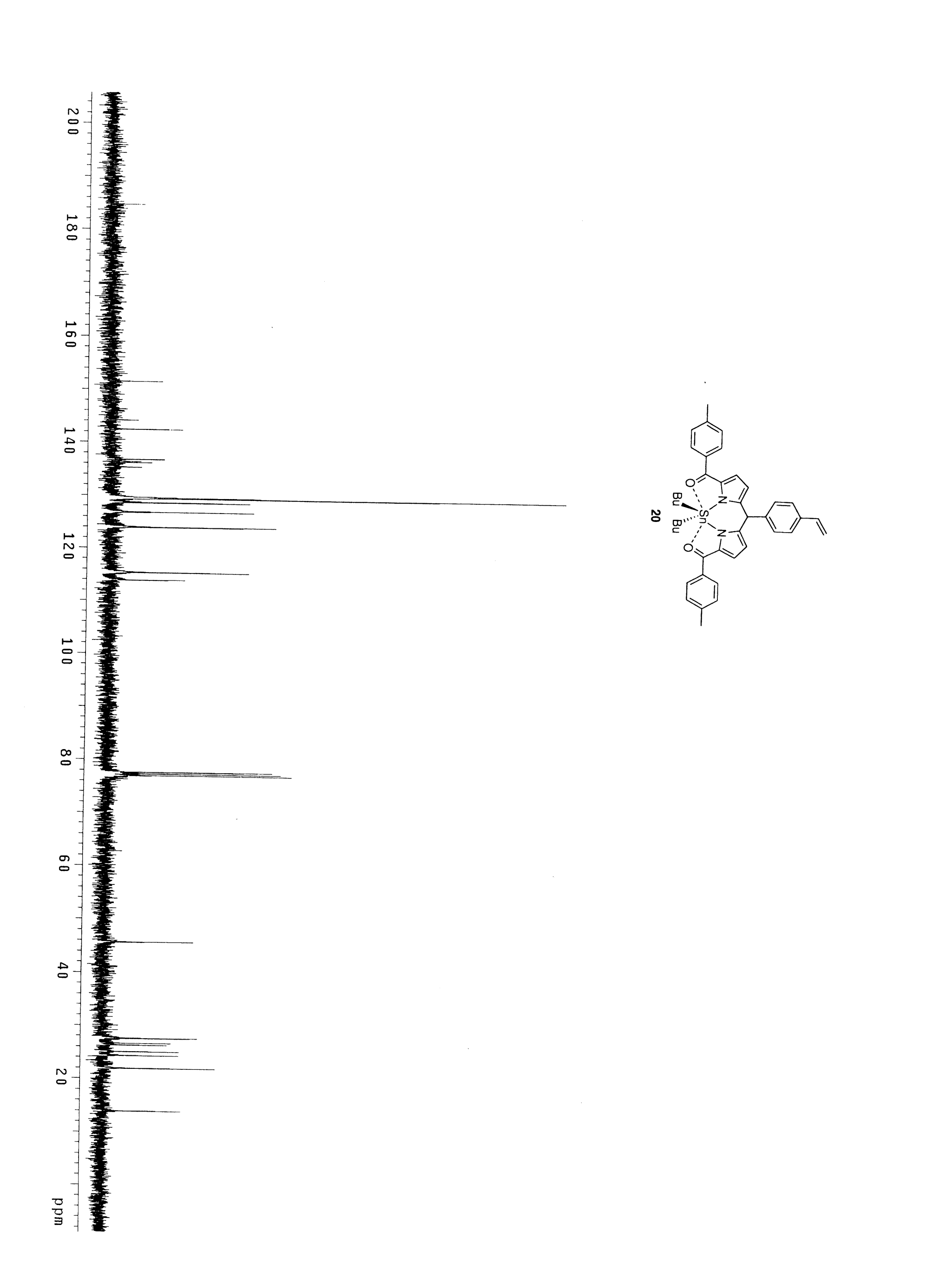




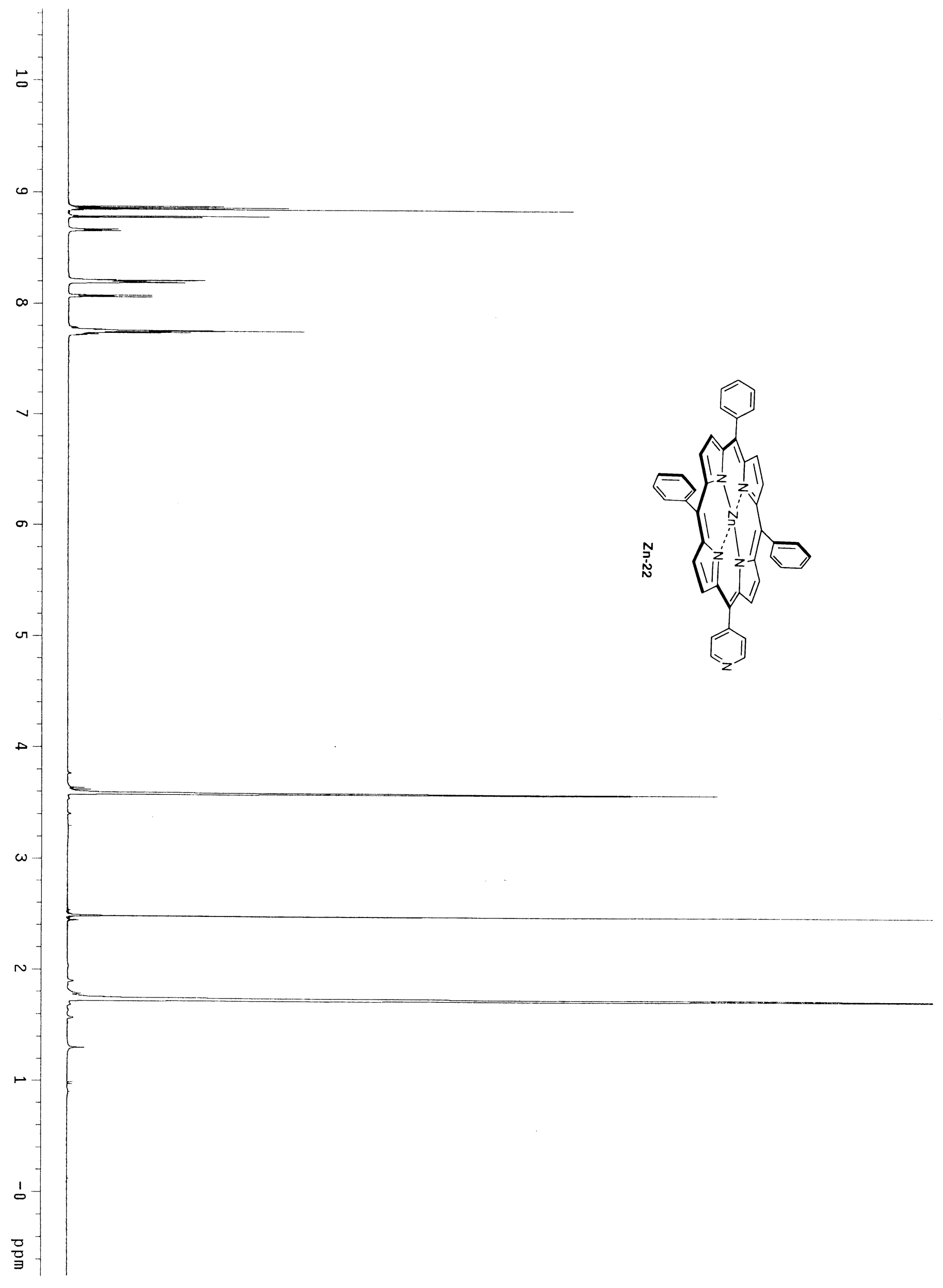



○
$\begin{array}{ll}N & 0 \\ O & O \\ O & O\end{array}$
$\begin{array}{ll}0 & \infty \\ 0 & 0 \\ 0 & 0\end{array}$
$\overrightarrow{0}$
$\bigcirc$
$\bigcirc$
$\vec{N}$
$O$
$O$

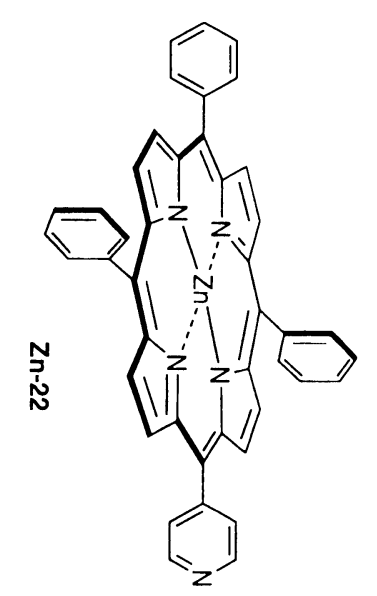

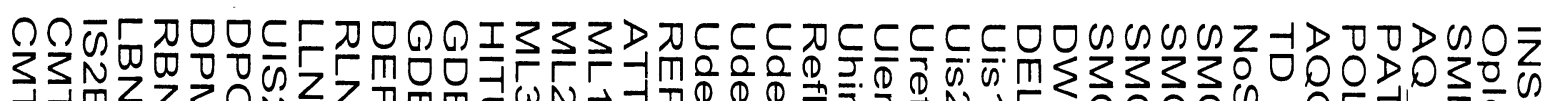

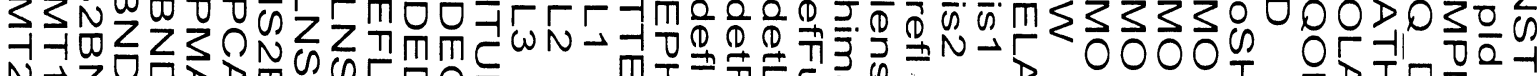

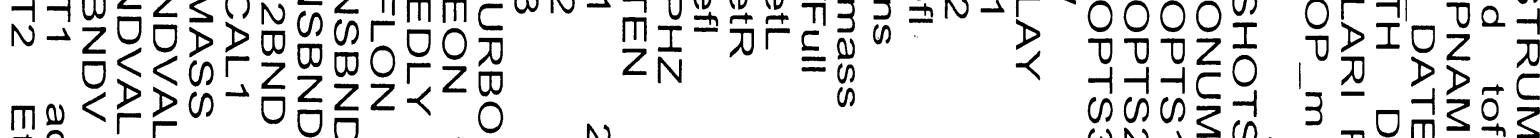

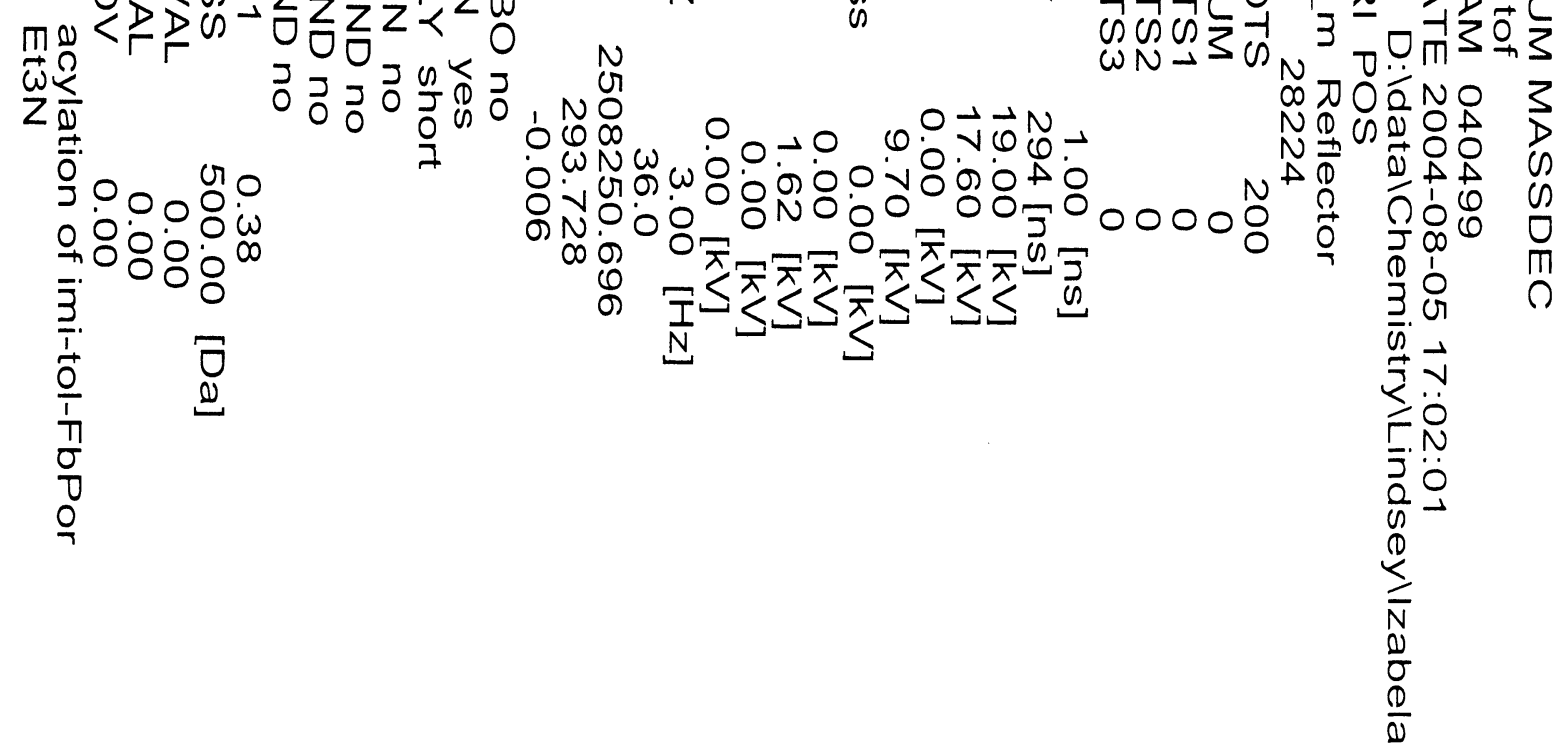




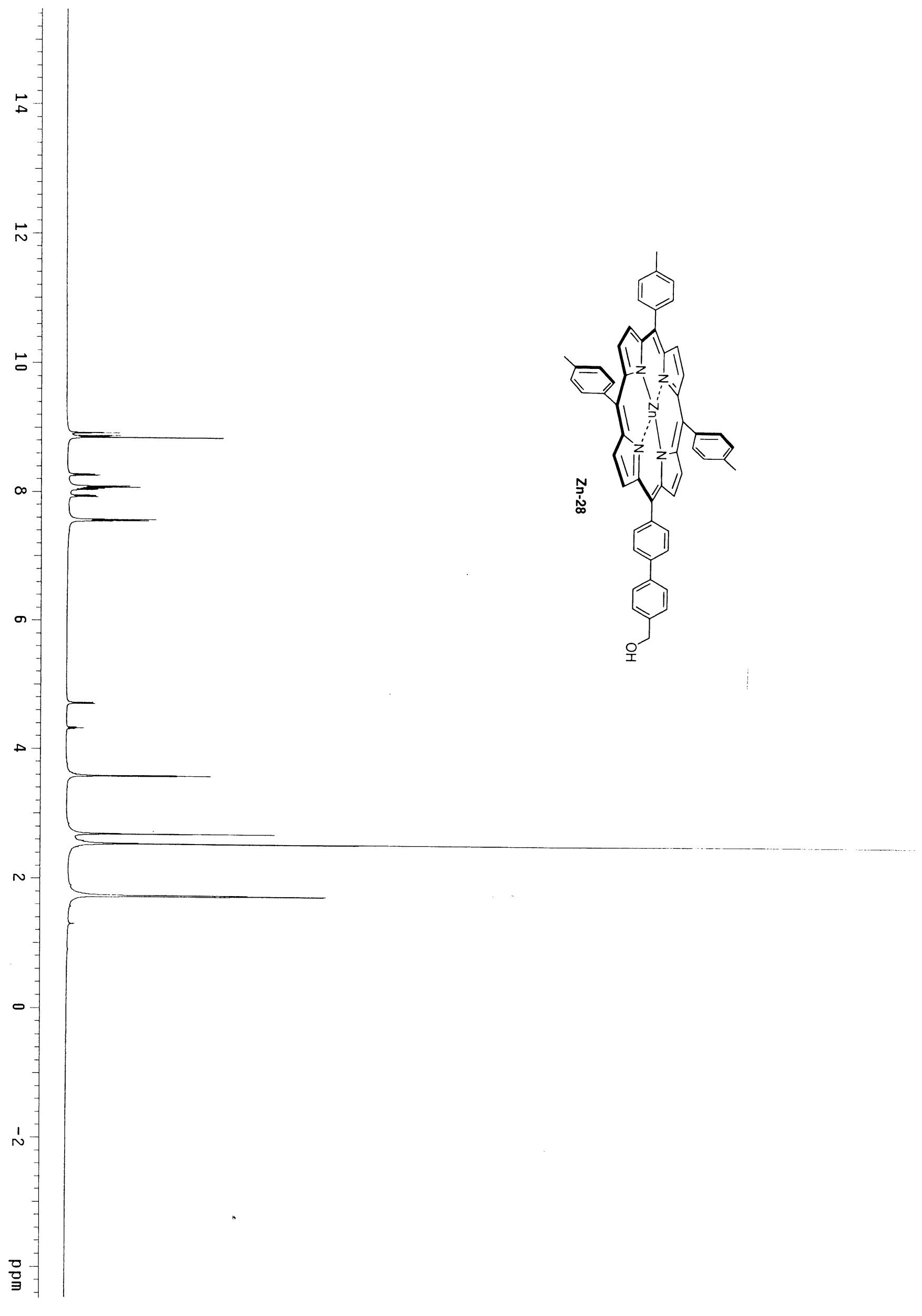




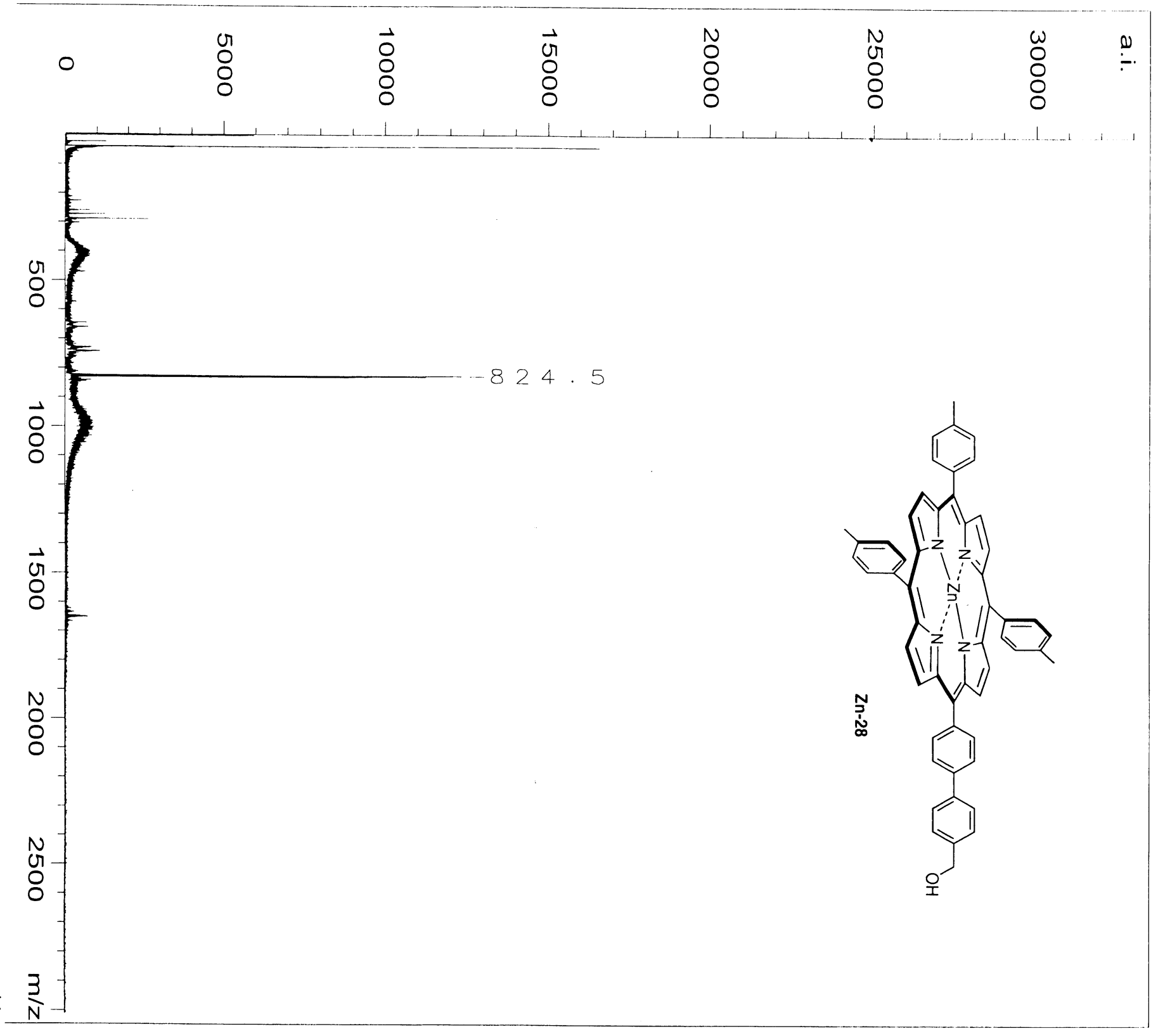

ภคต

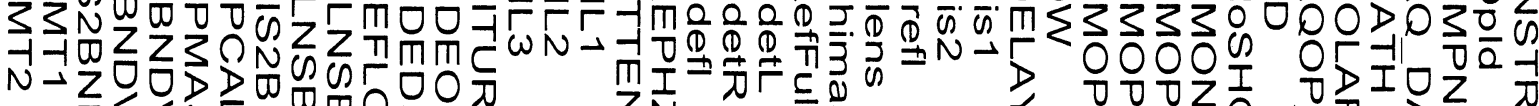

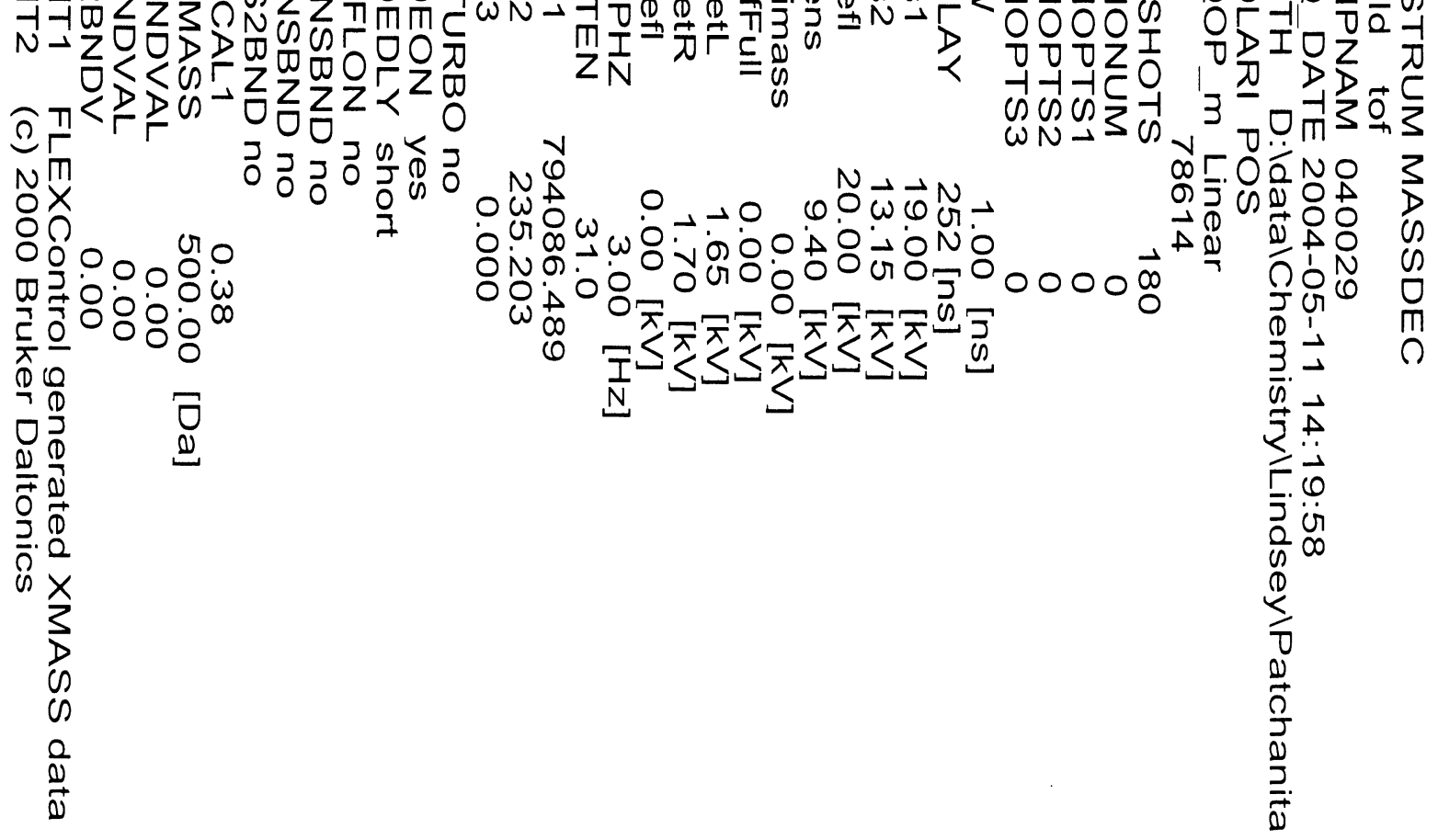




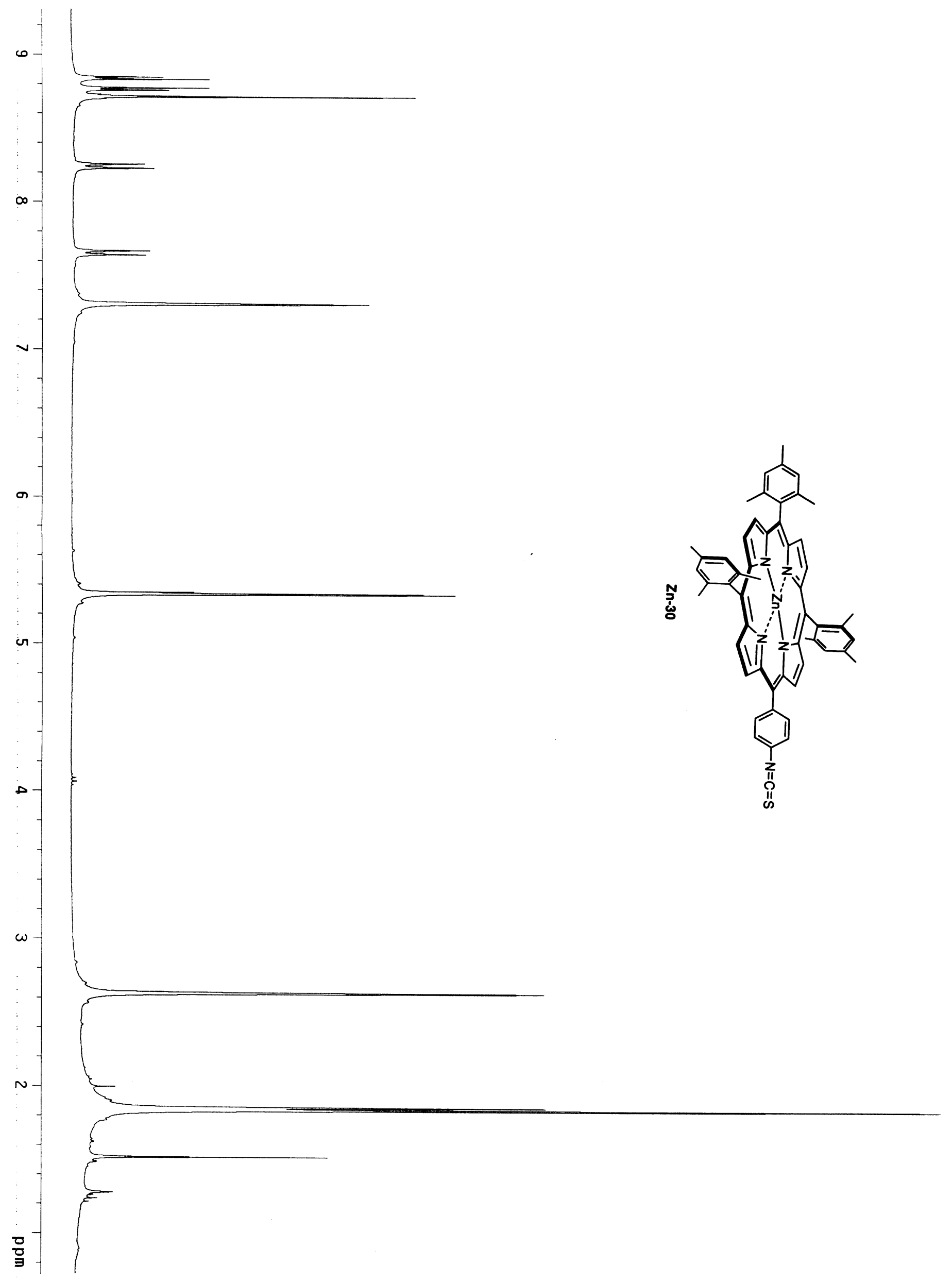




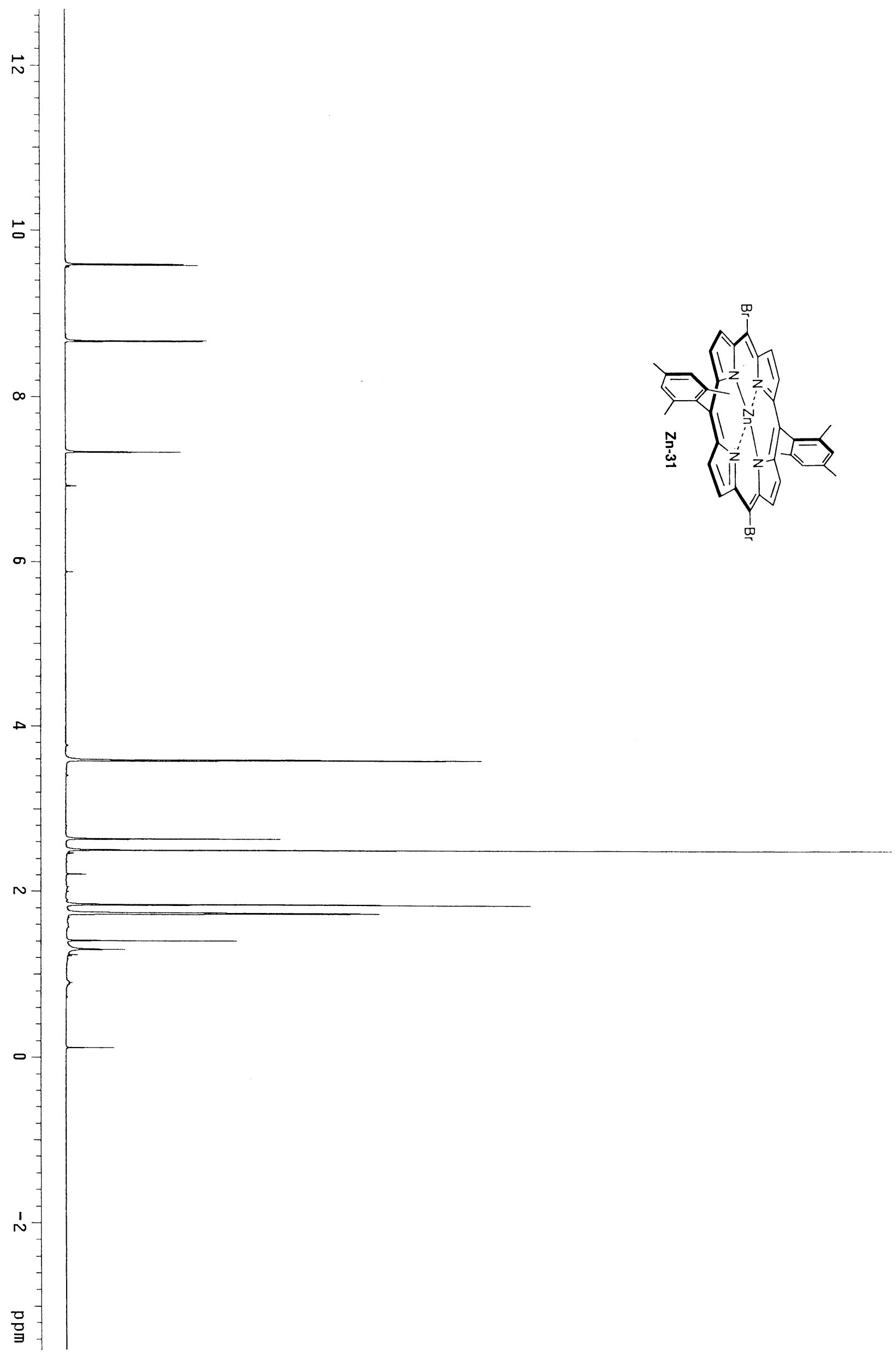




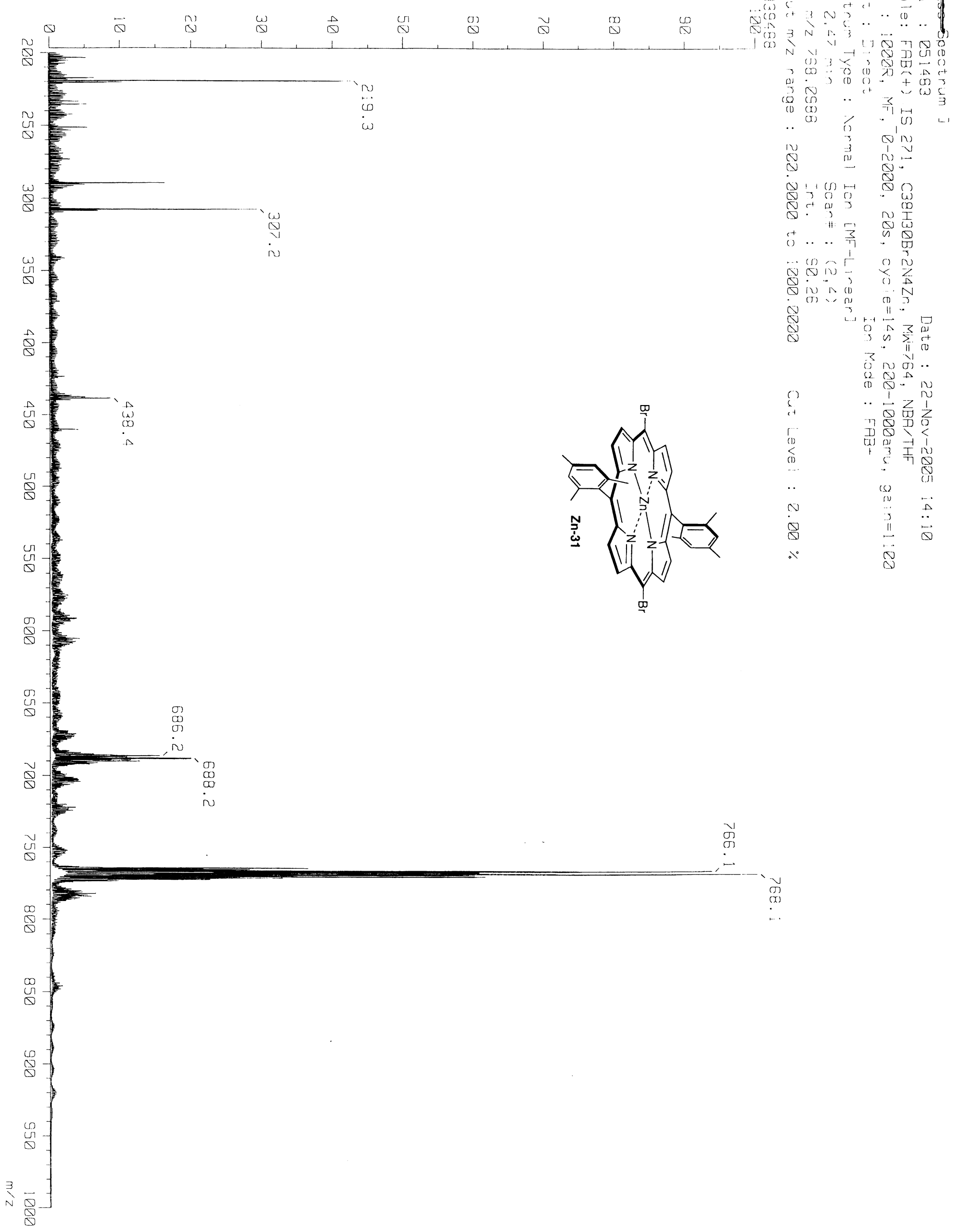




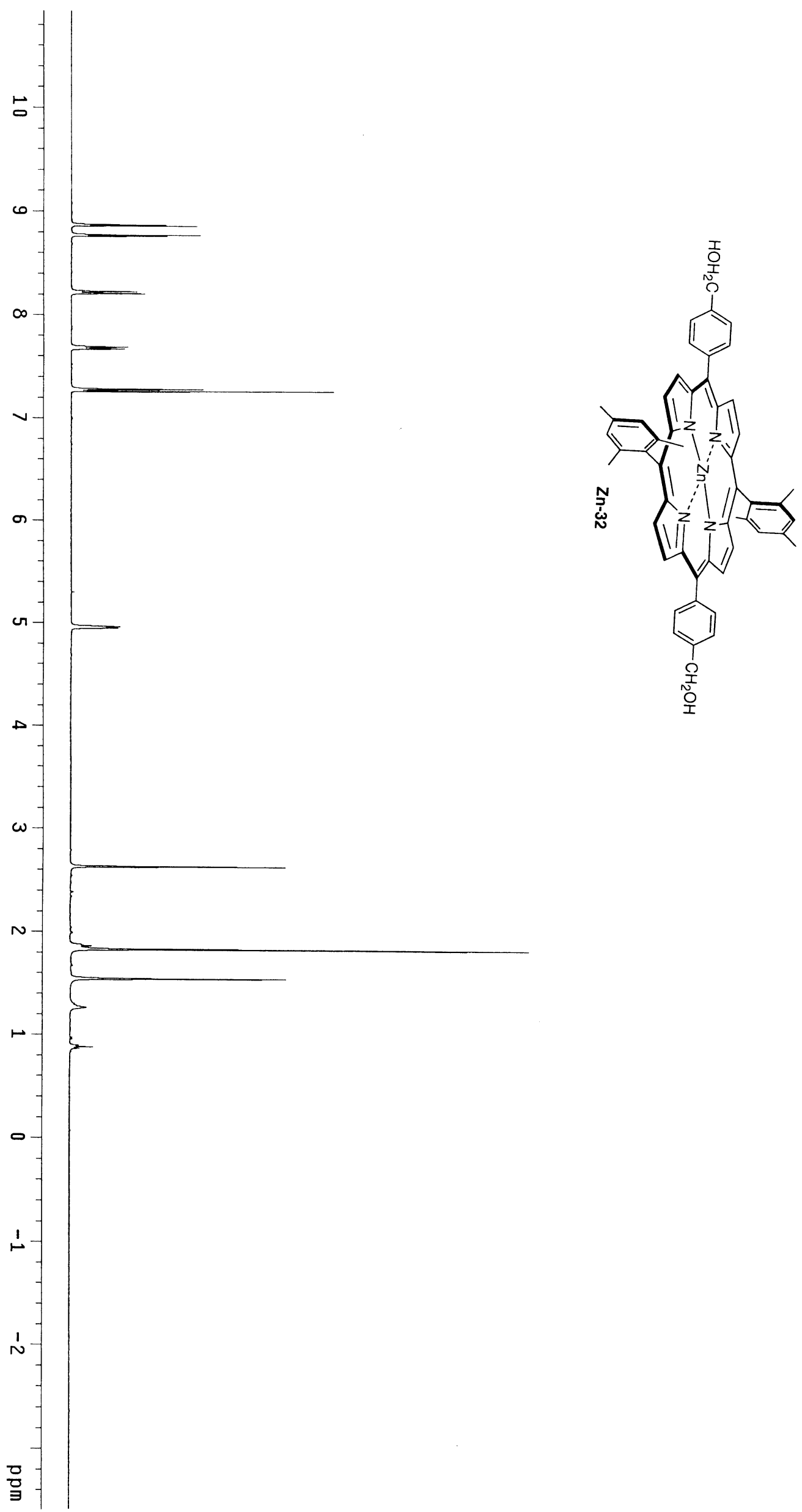




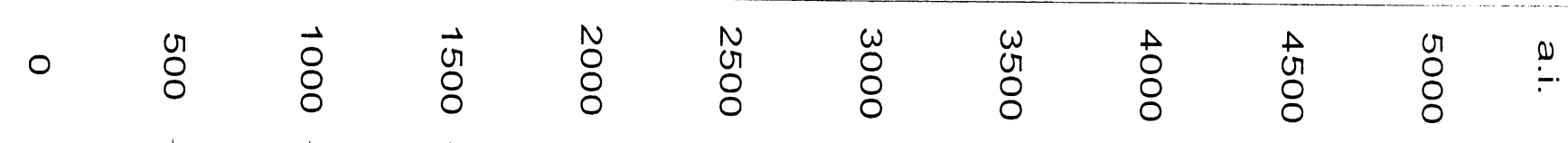

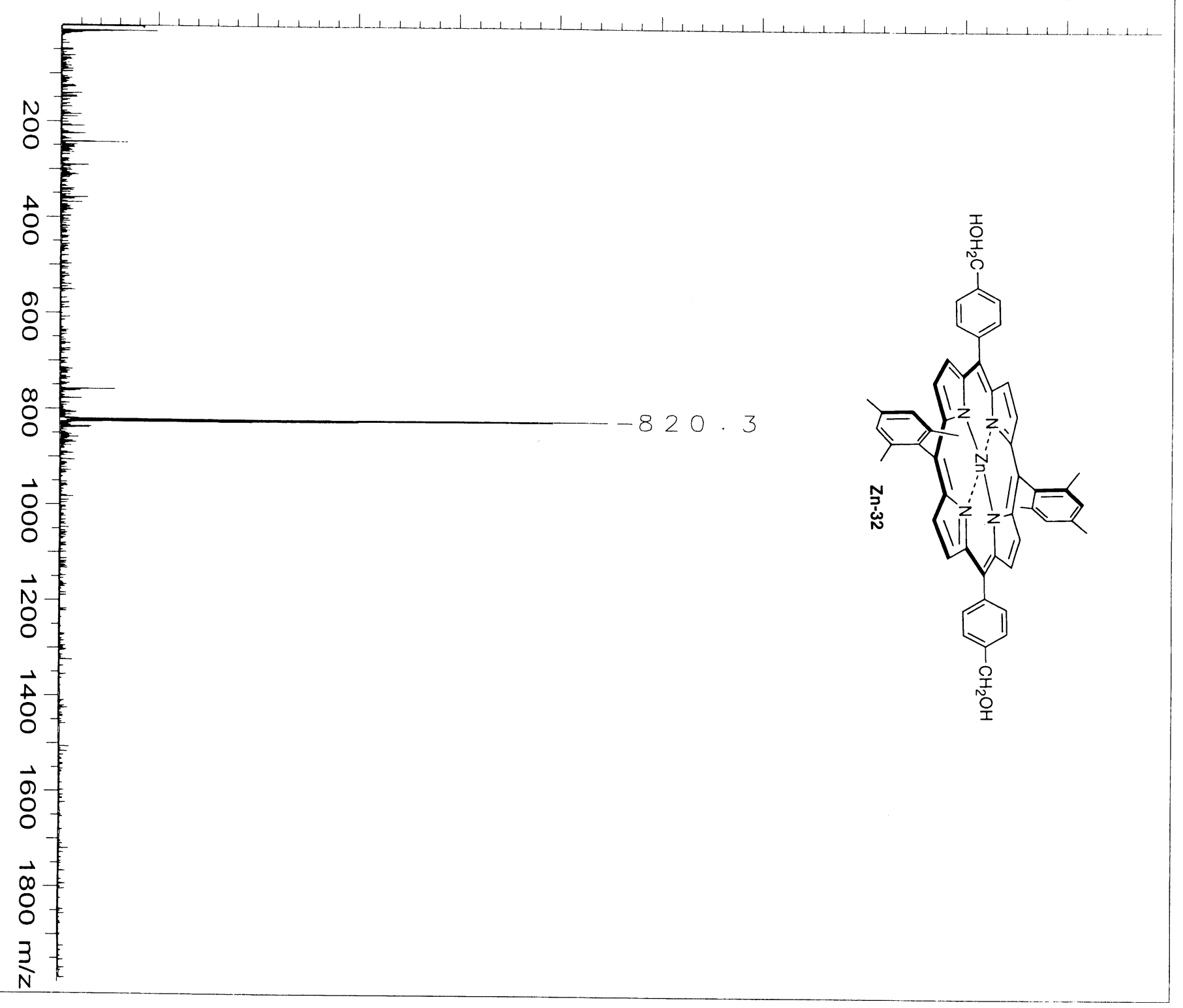

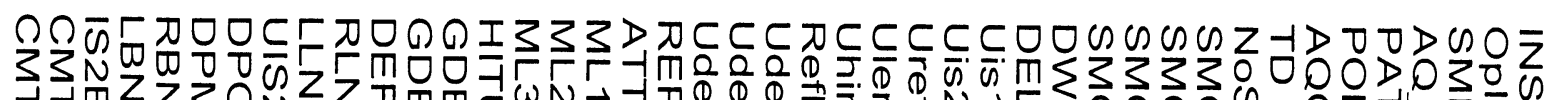

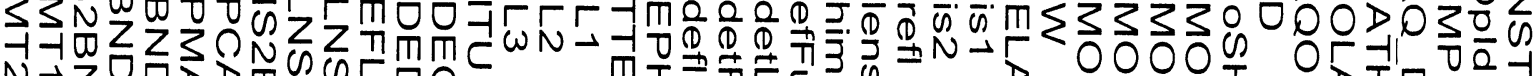

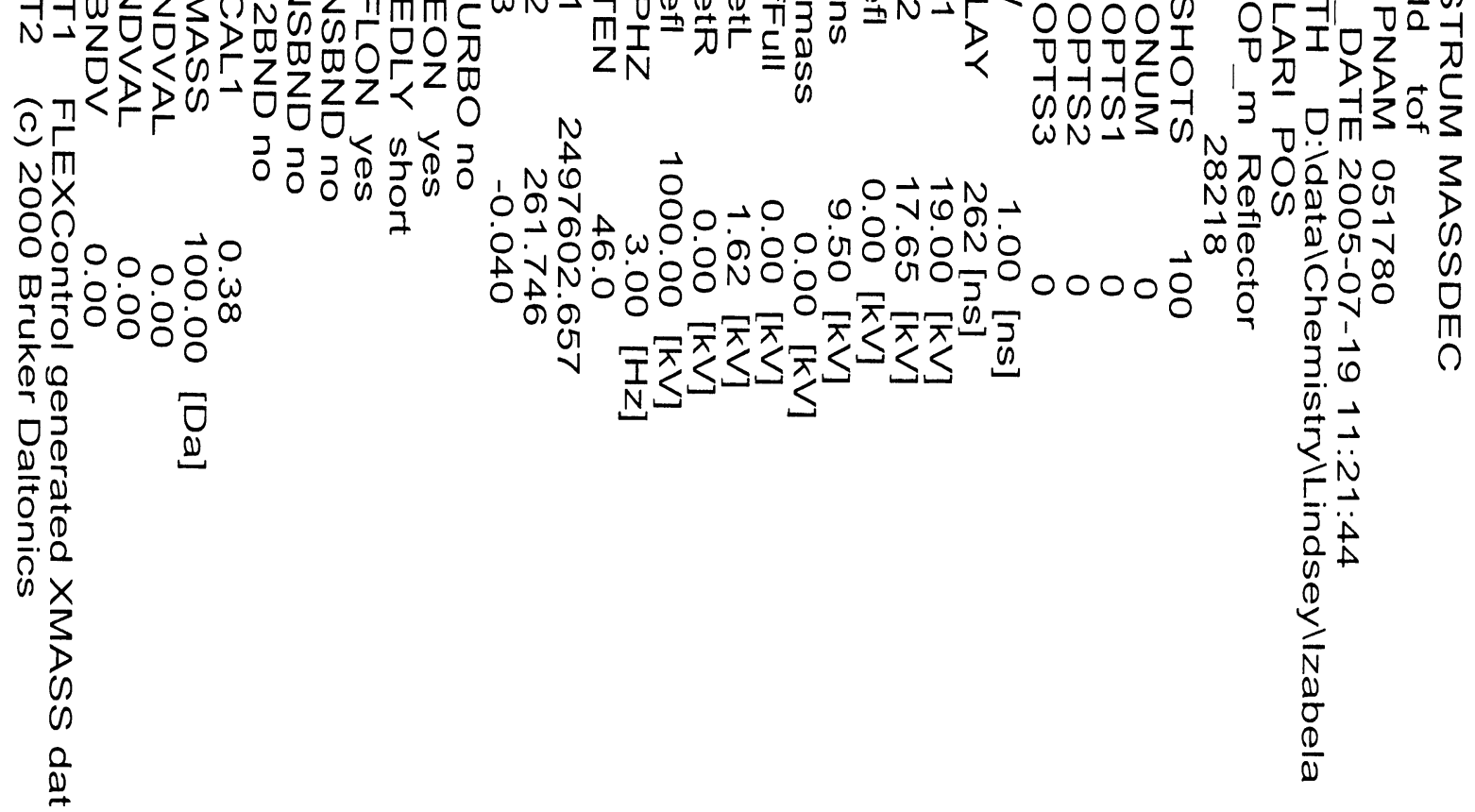




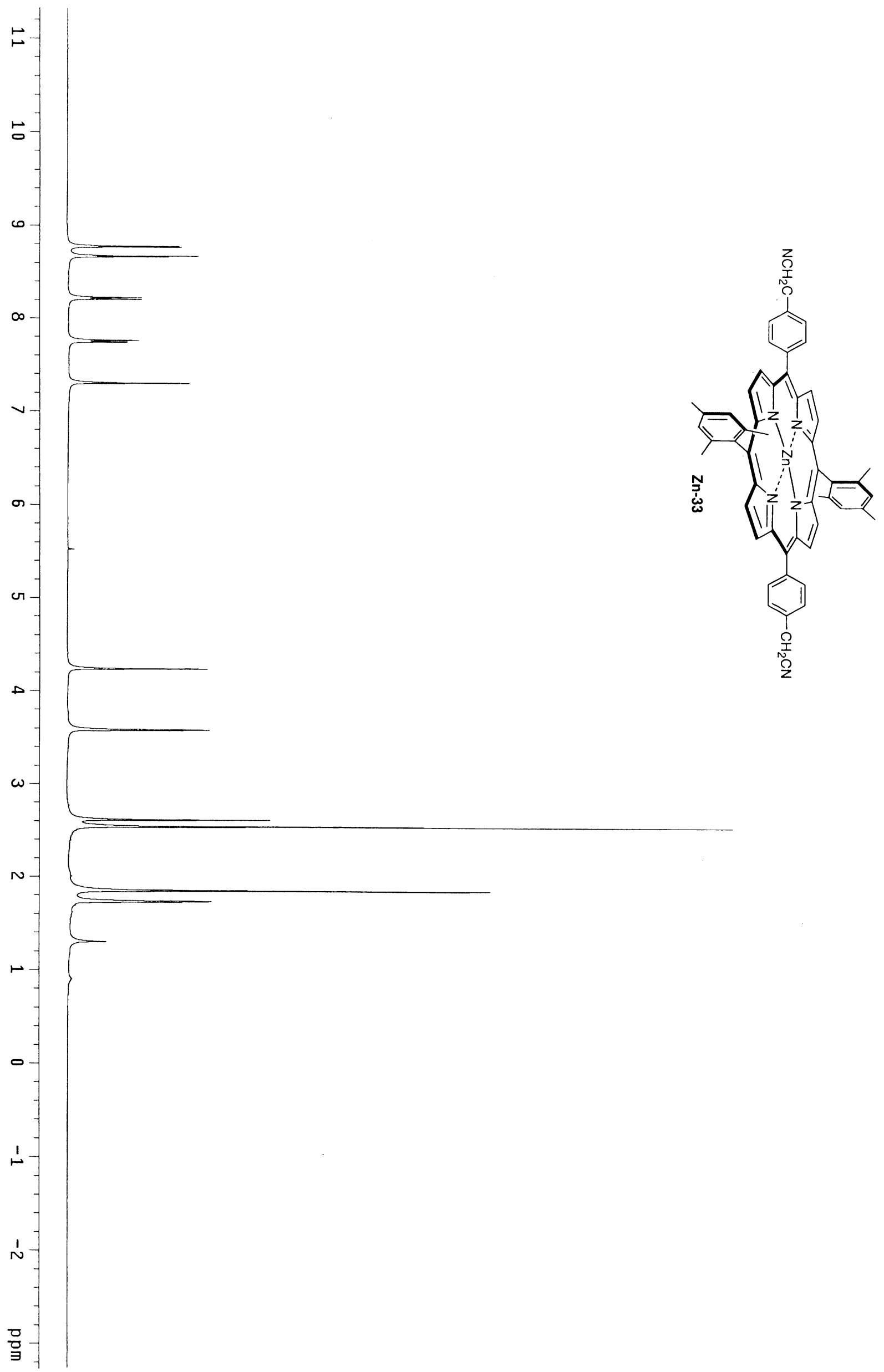




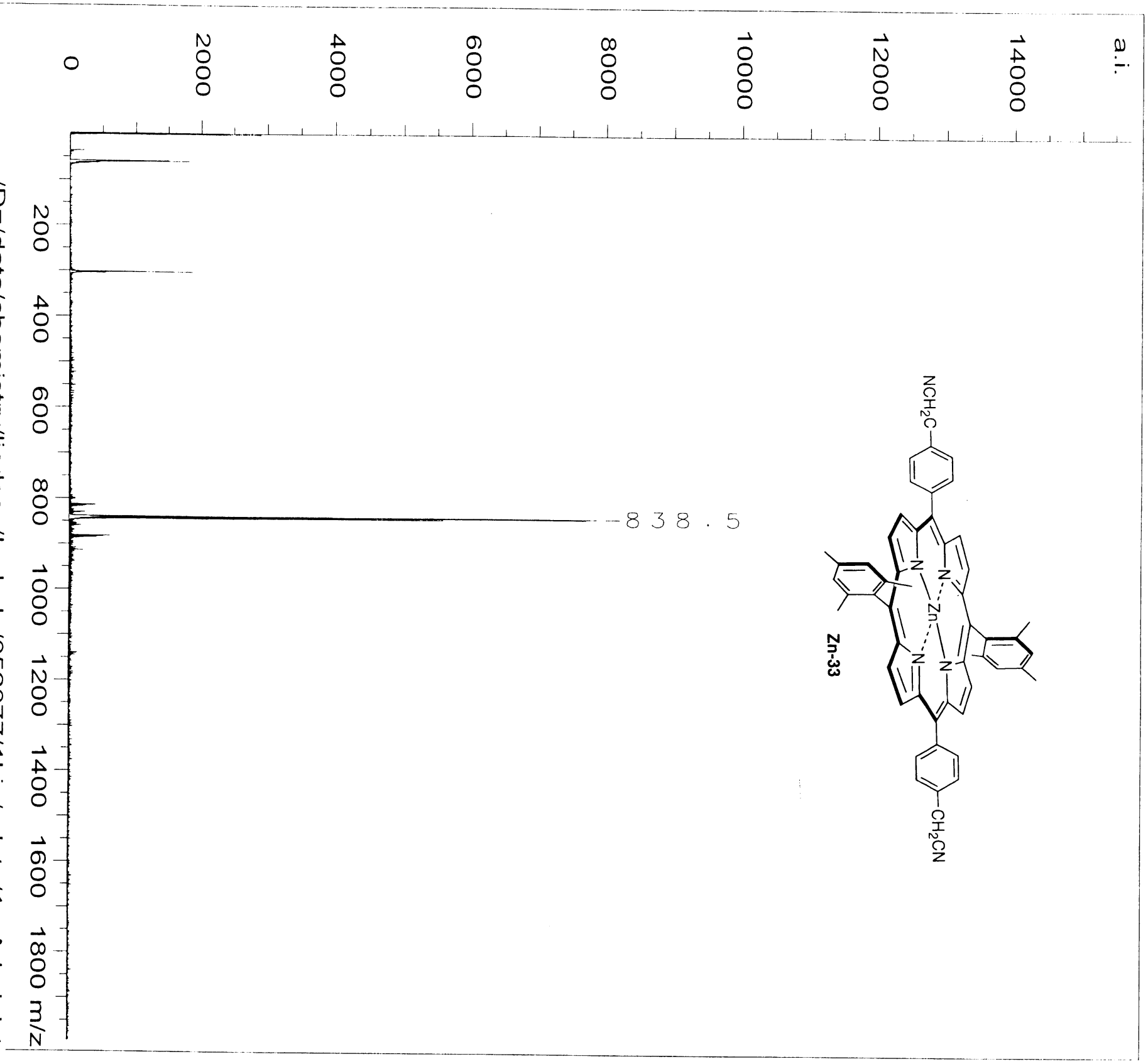

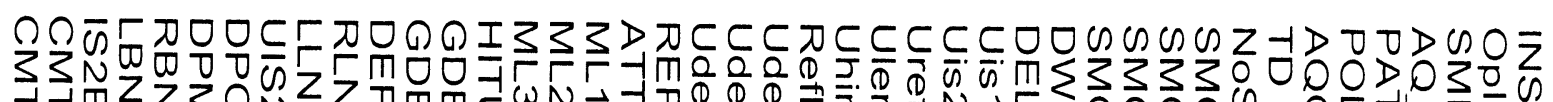

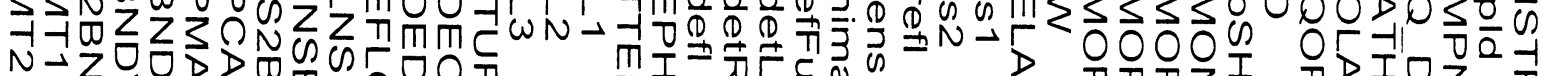

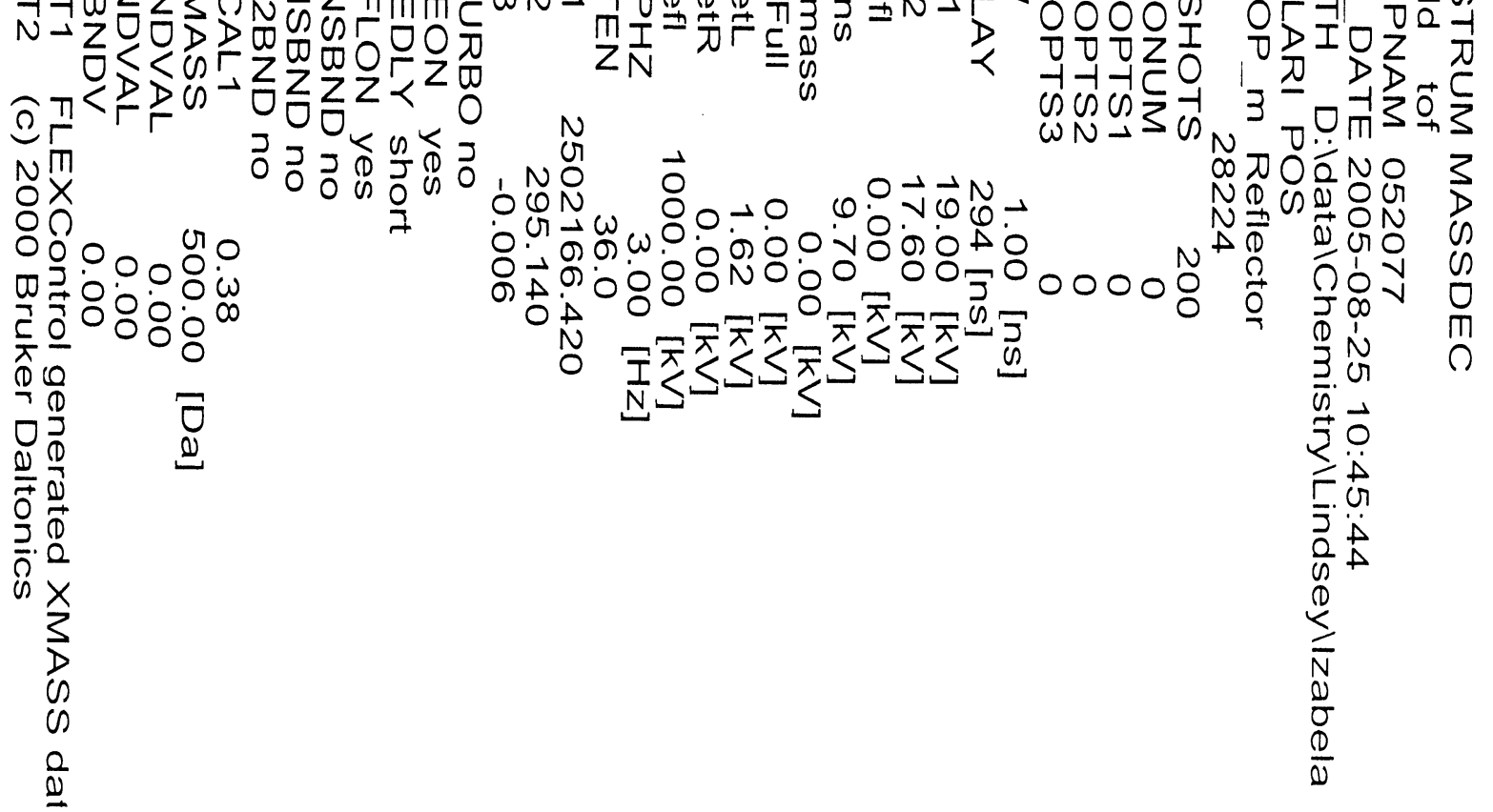




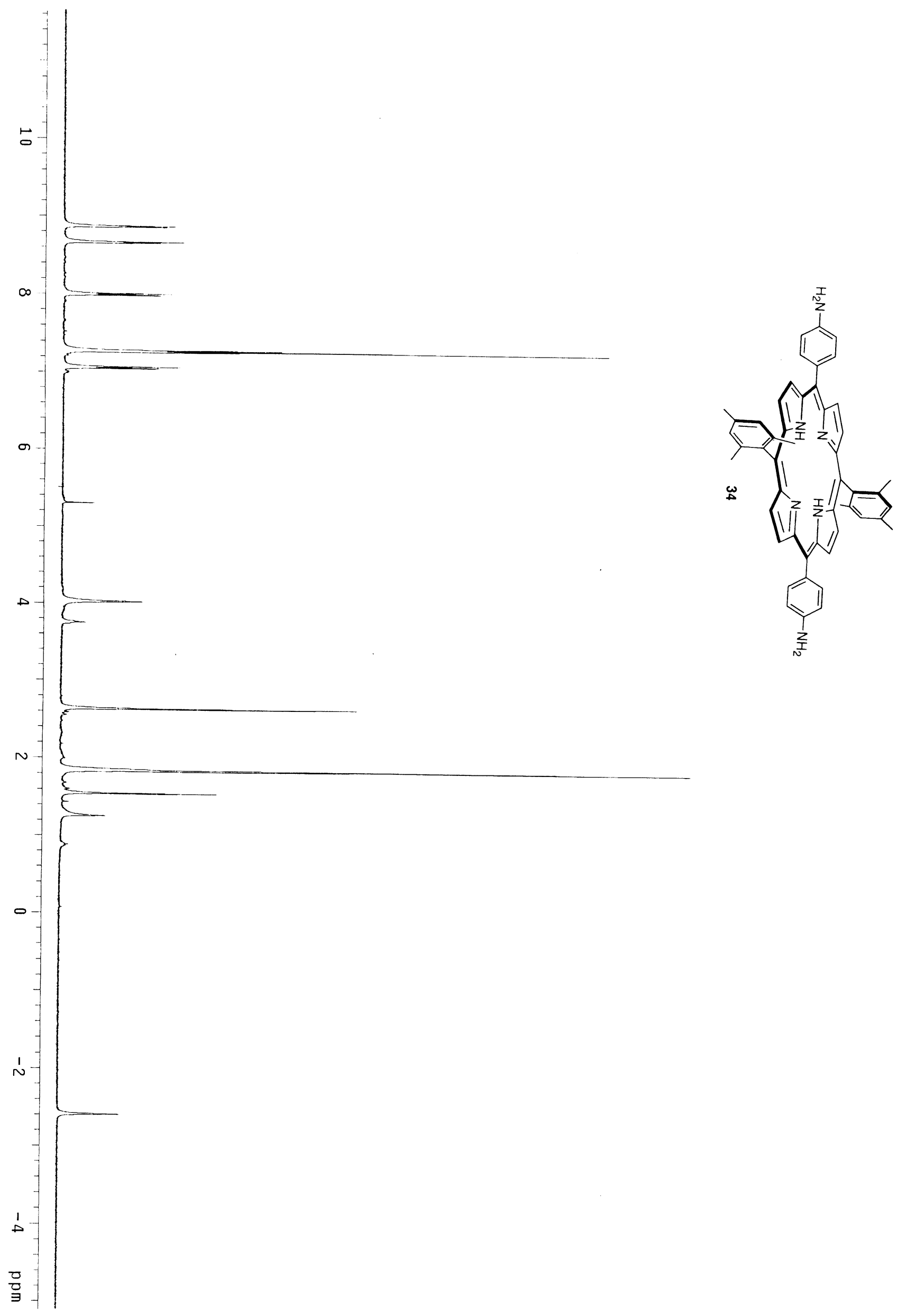


- $\quad:$

离 $\quad \vec{\square}$

ัํำ

음

:

:

\&

$-728.9$

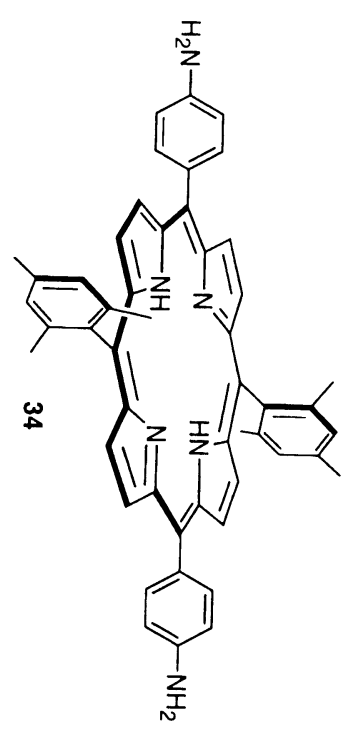

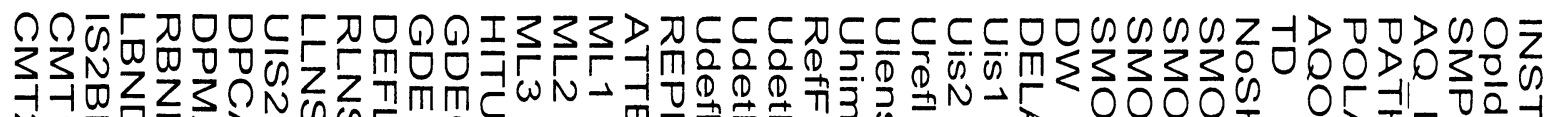

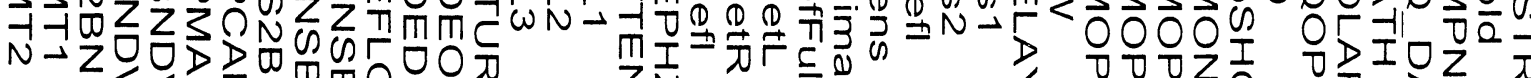

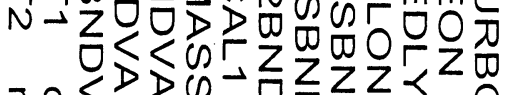

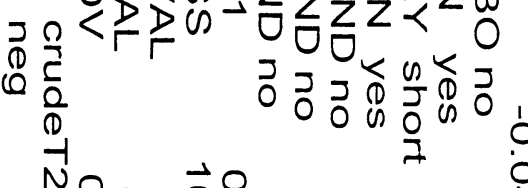

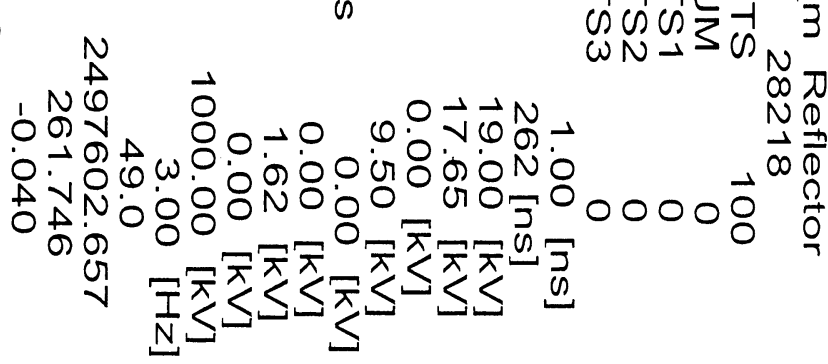

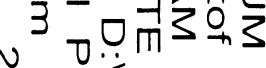

0<smiles>[AlH2]</smiles>

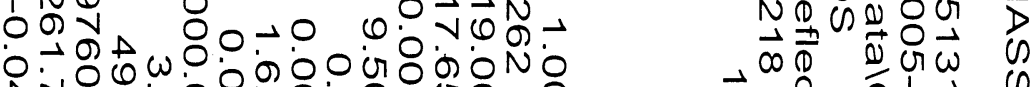

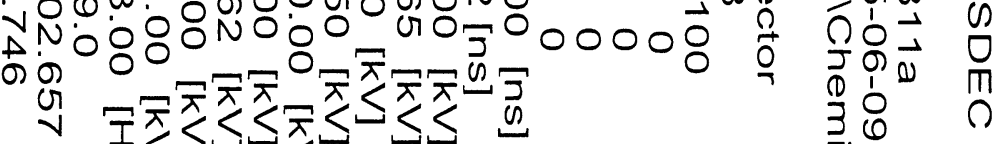
ฮั 


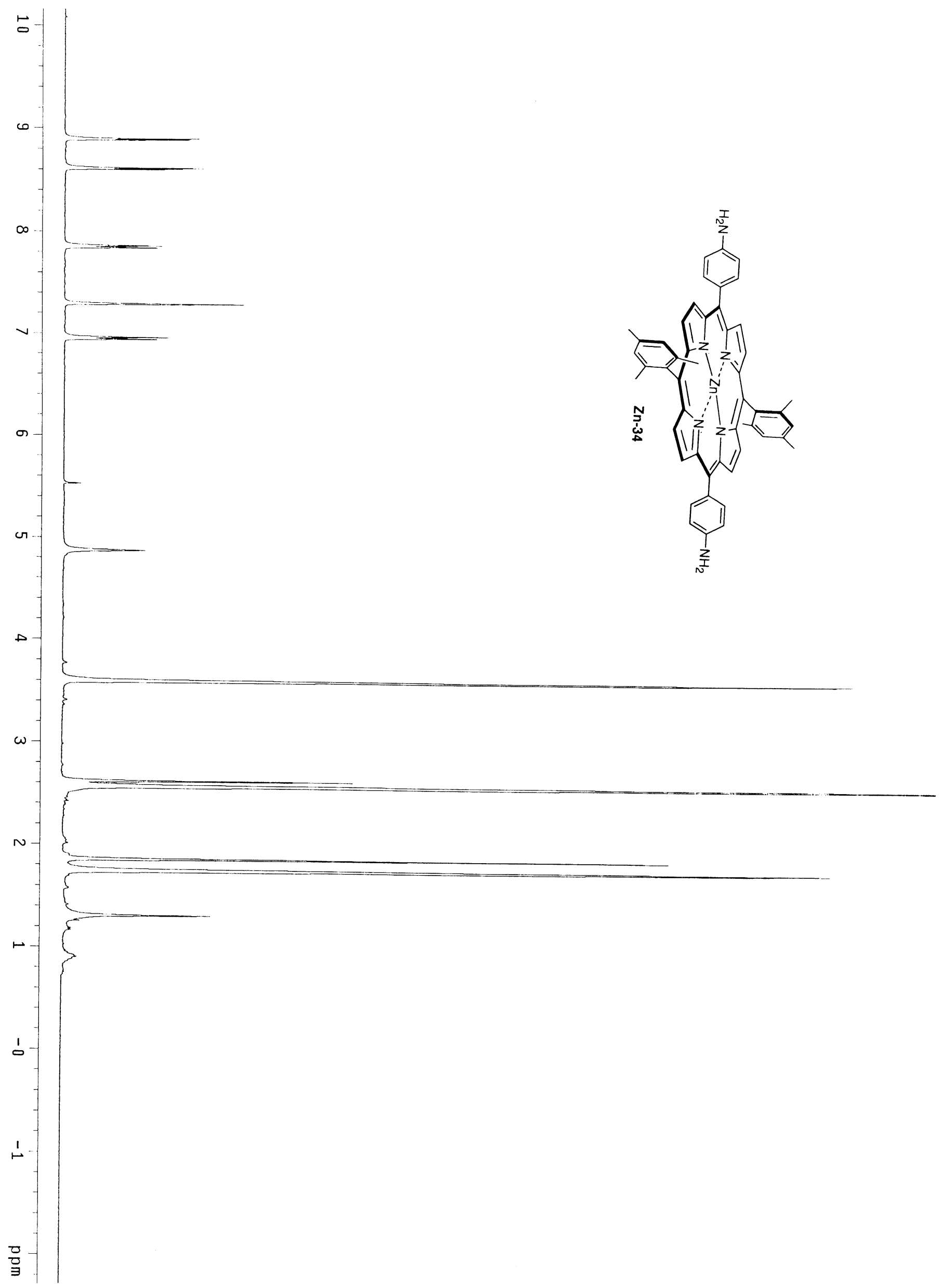



。
$\vec{\circ}$
$\overrightarrow{\mathrm{g}}$

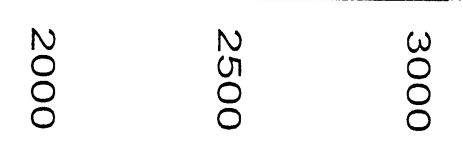
器
高

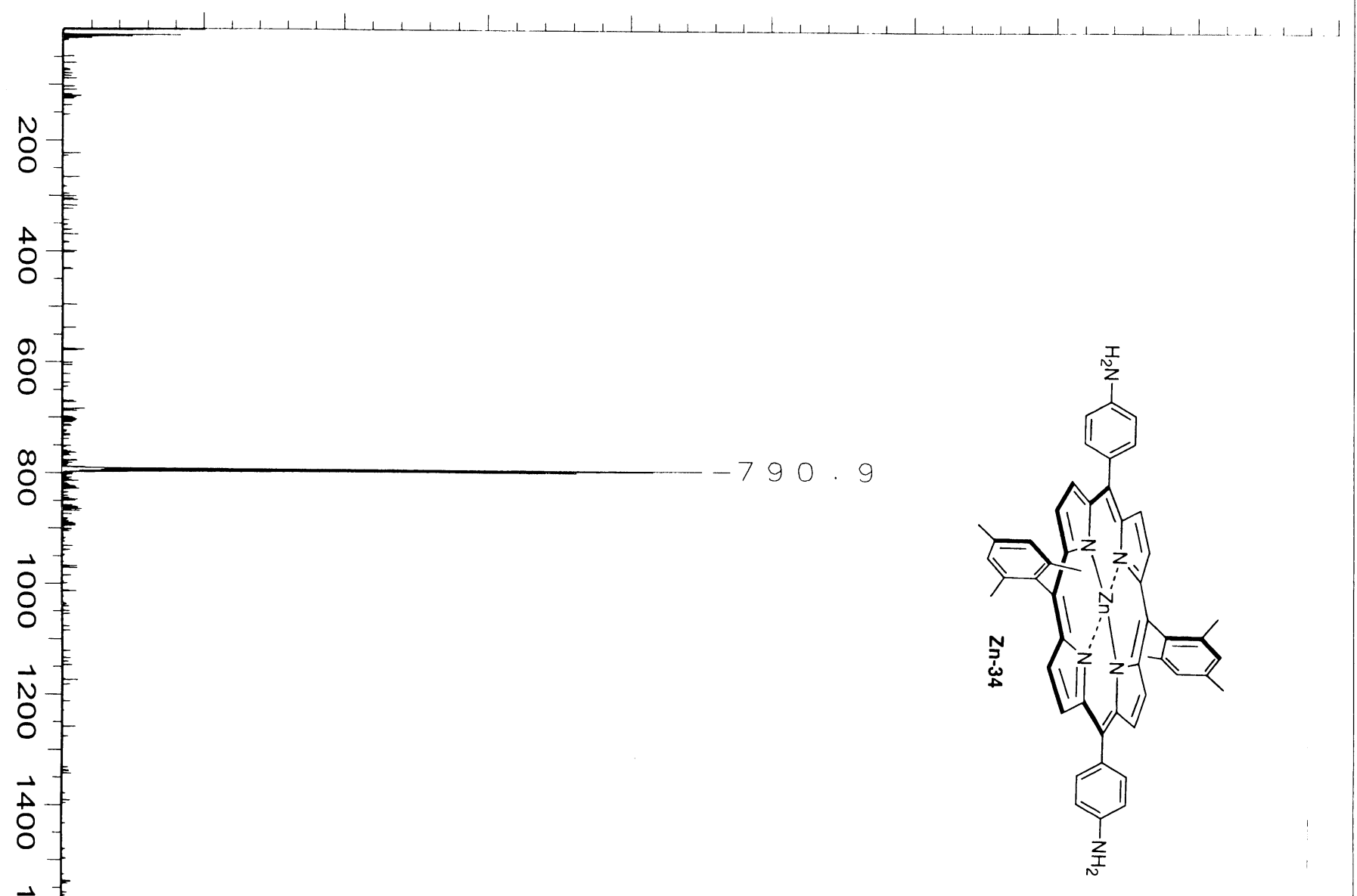

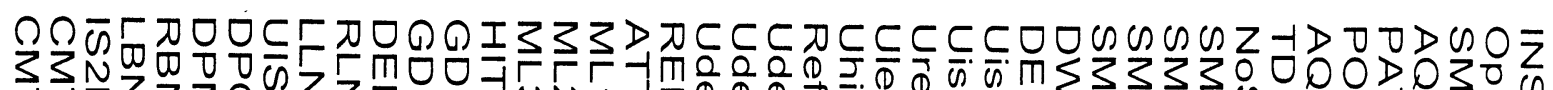

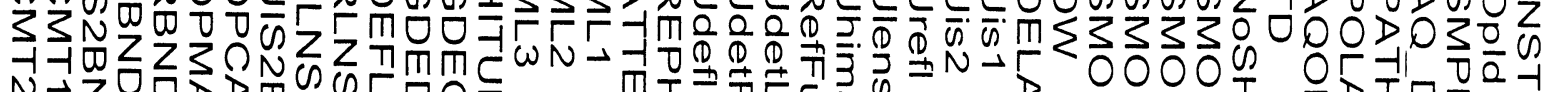

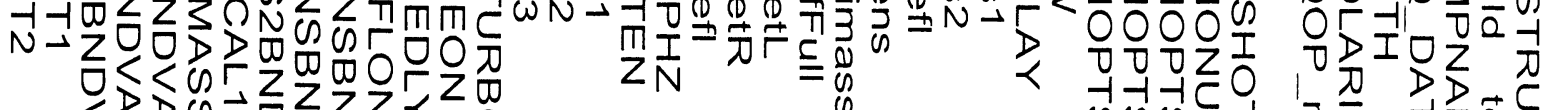

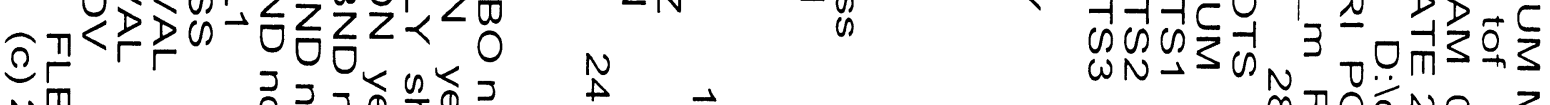

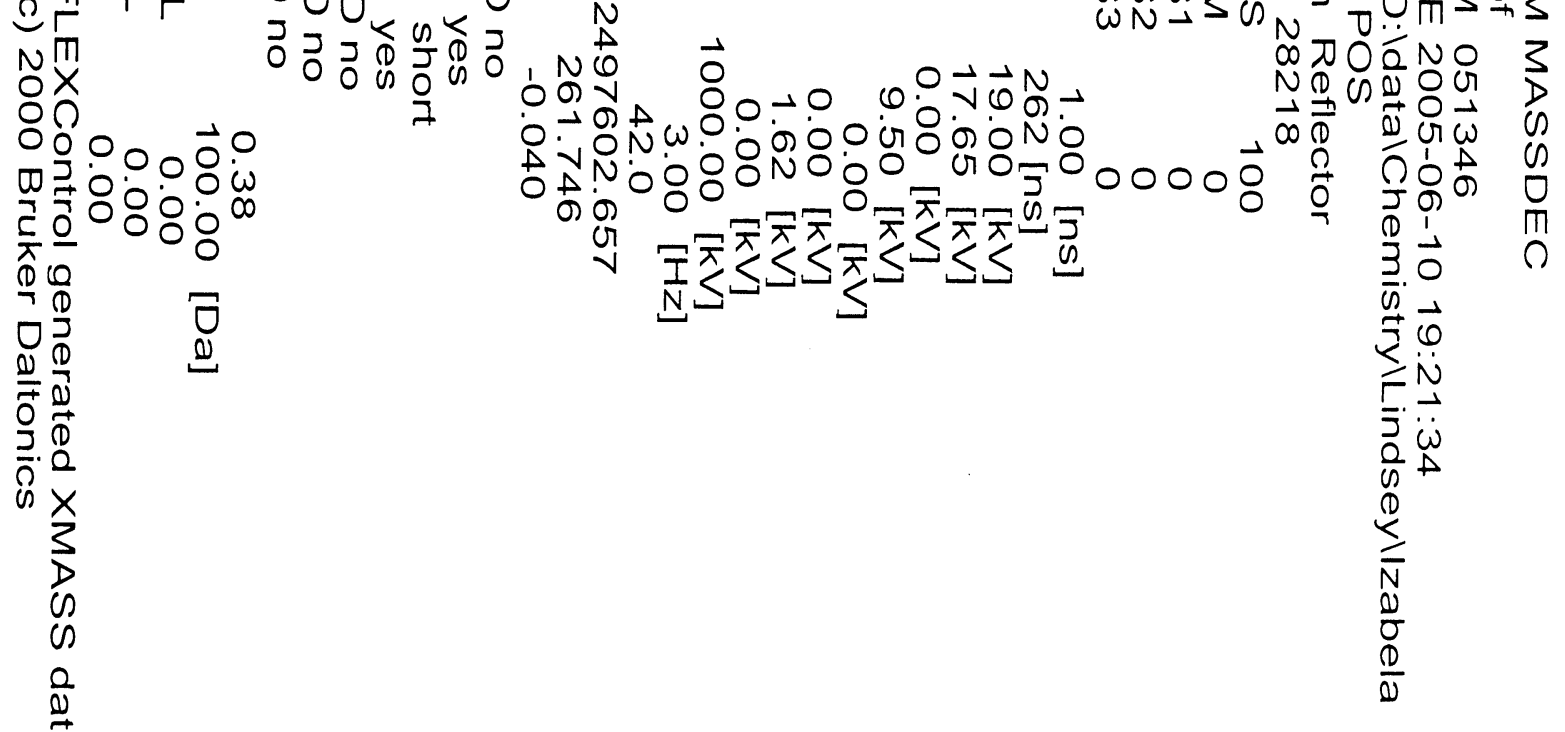




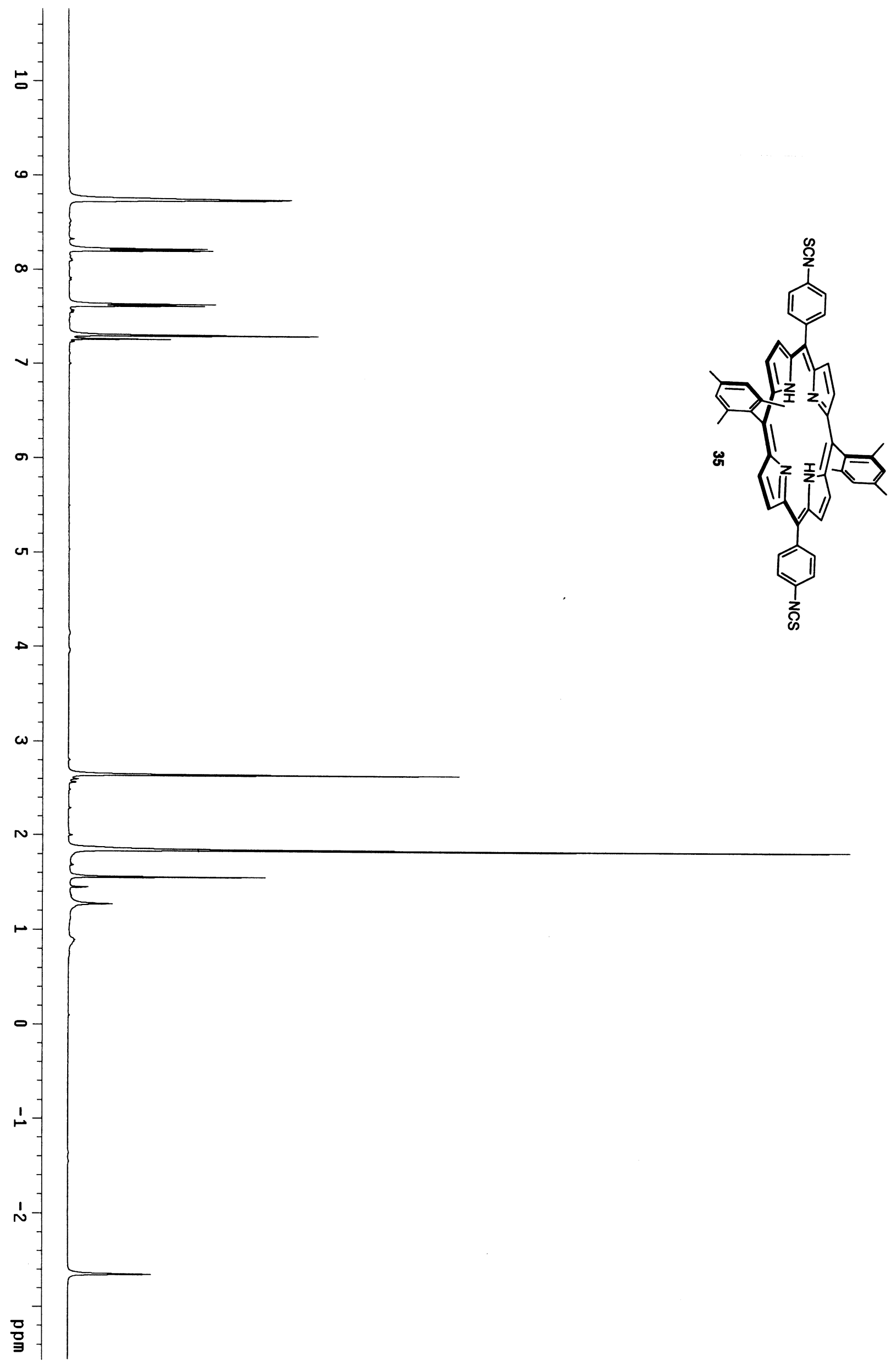



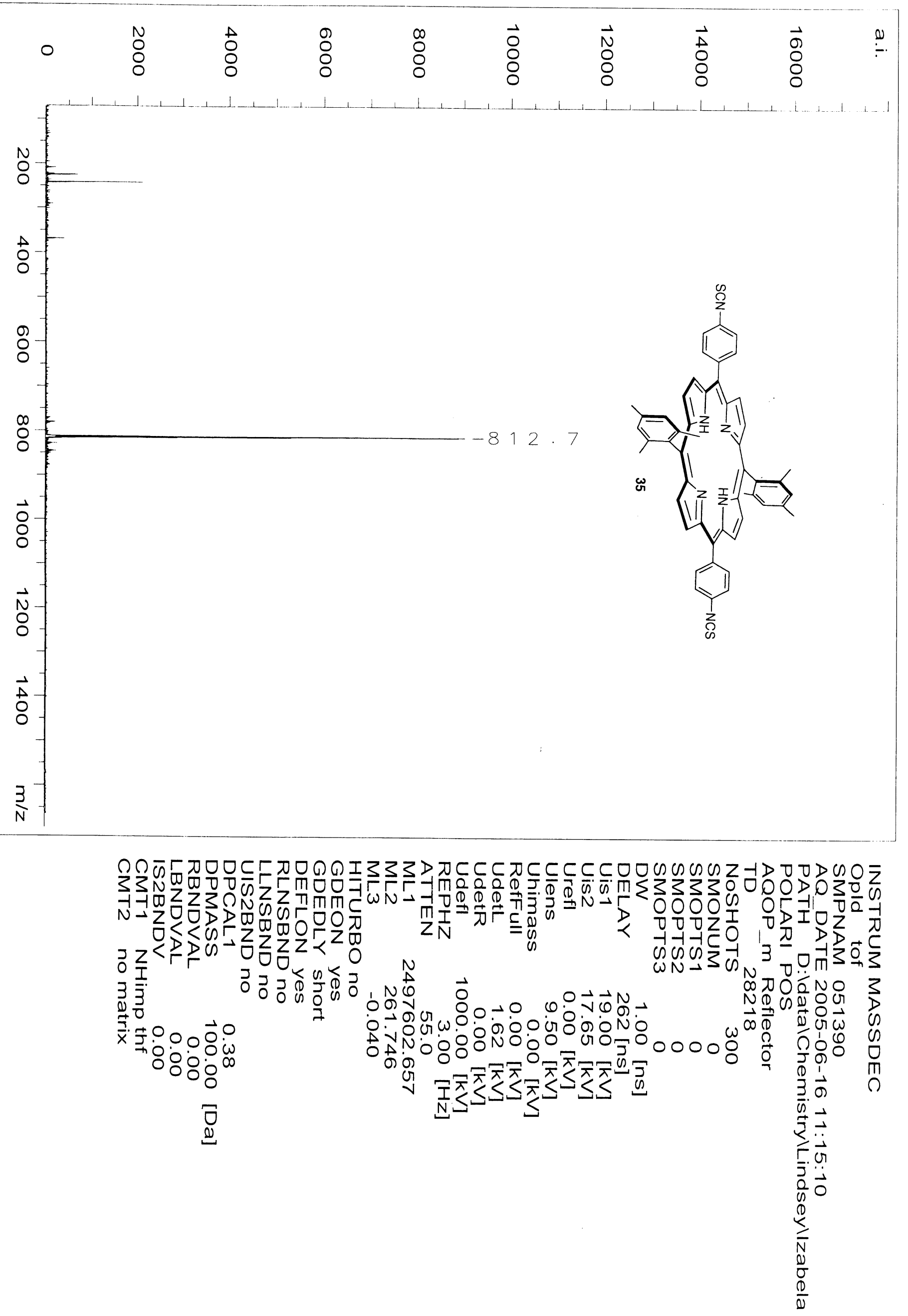


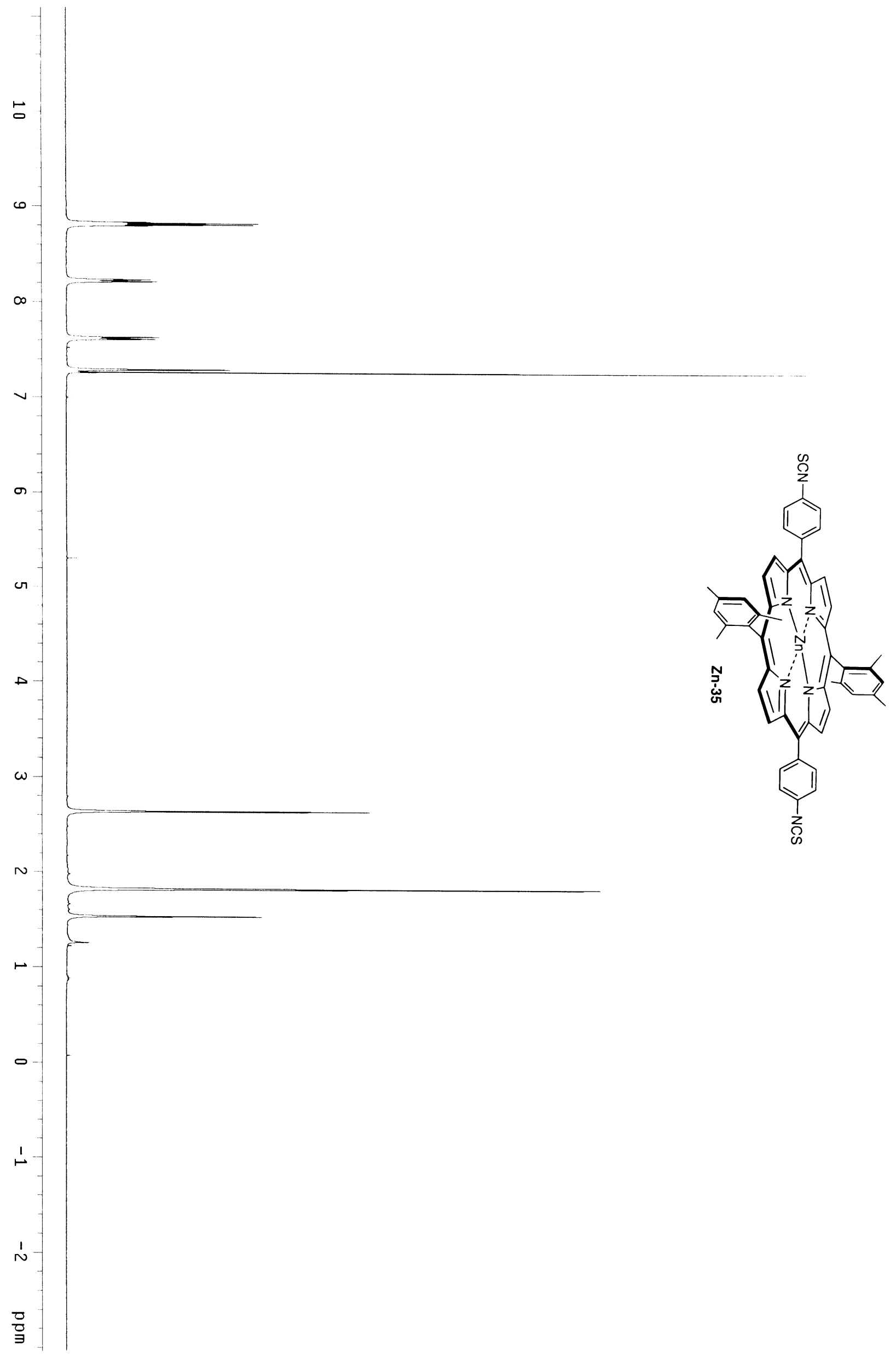




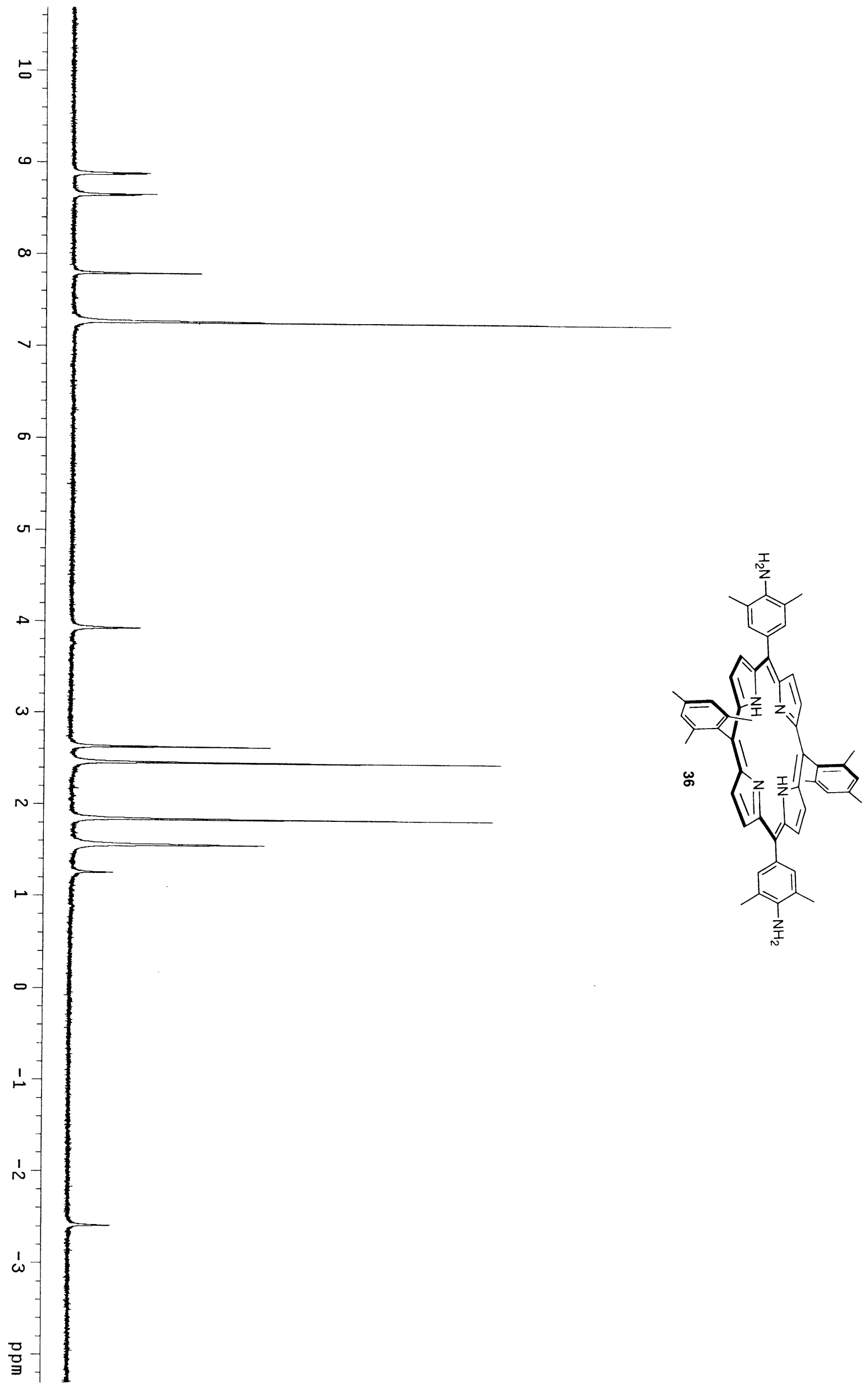




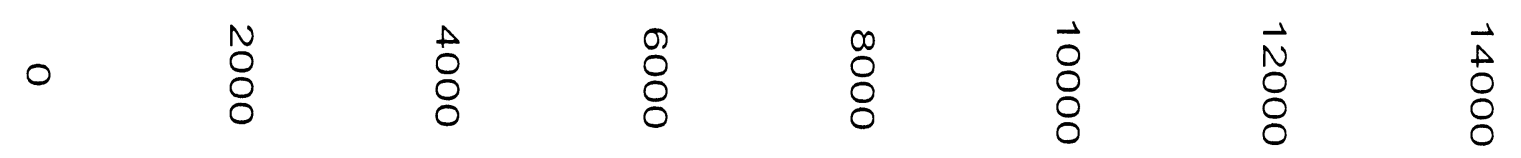

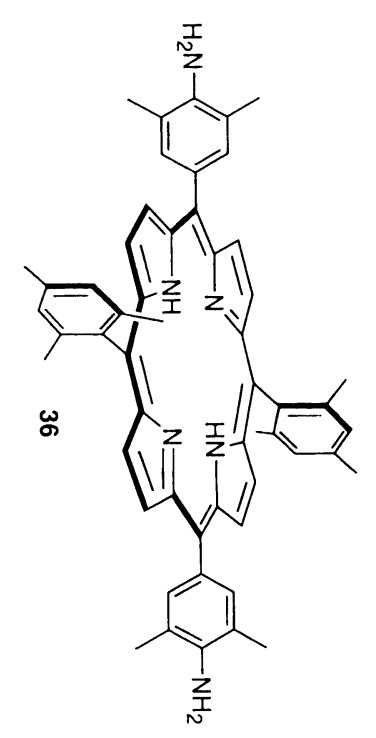

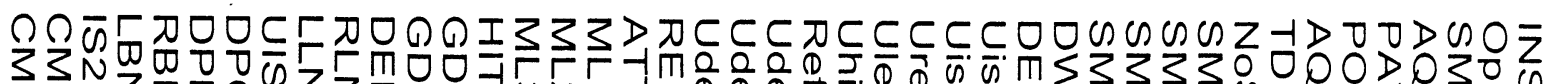

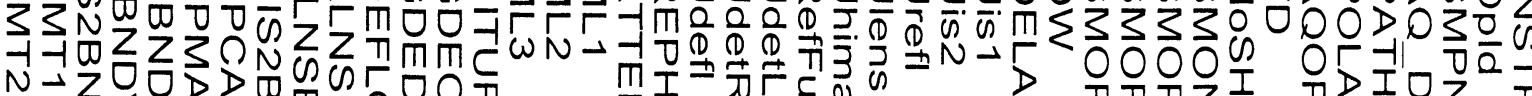

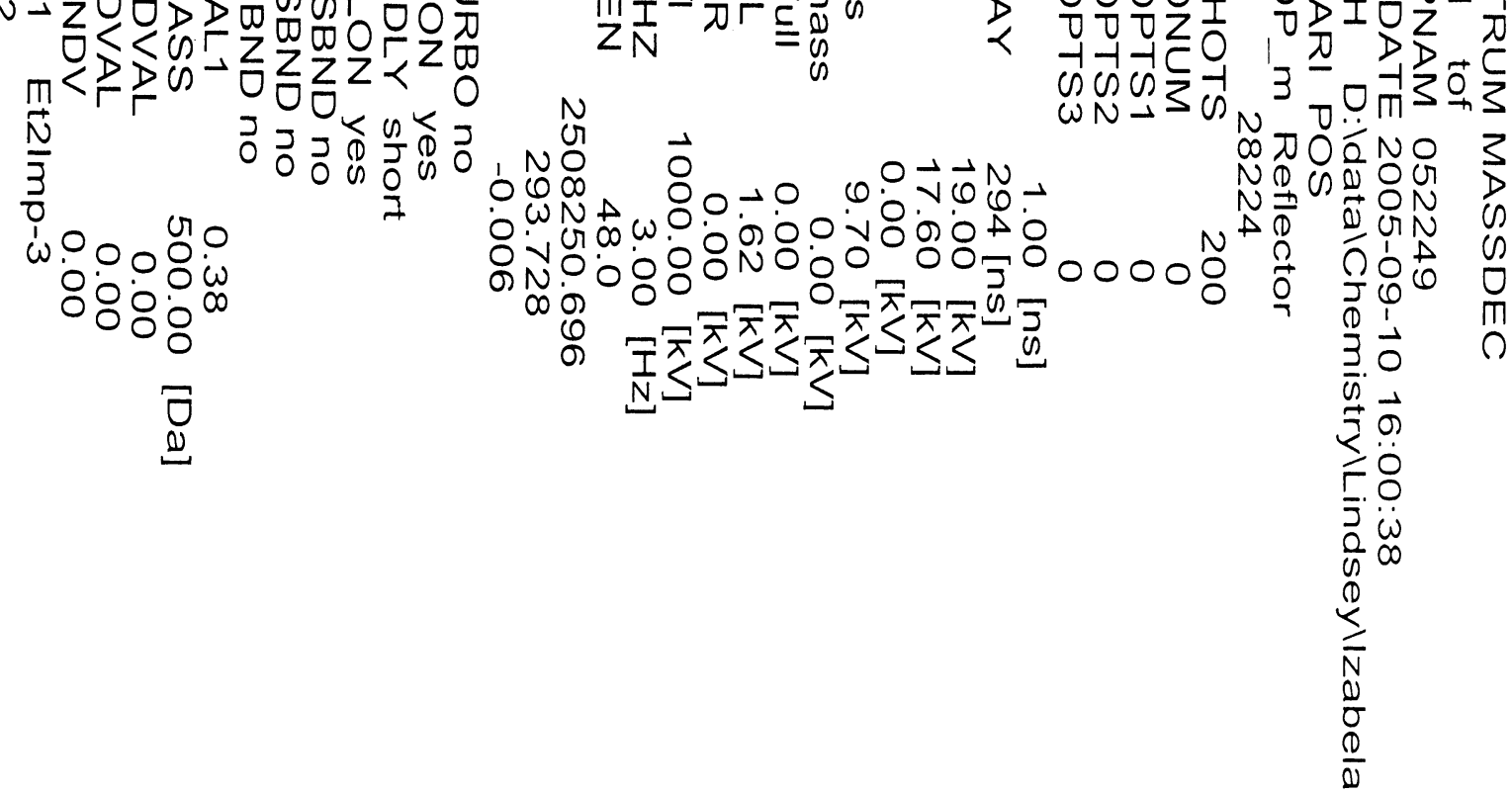




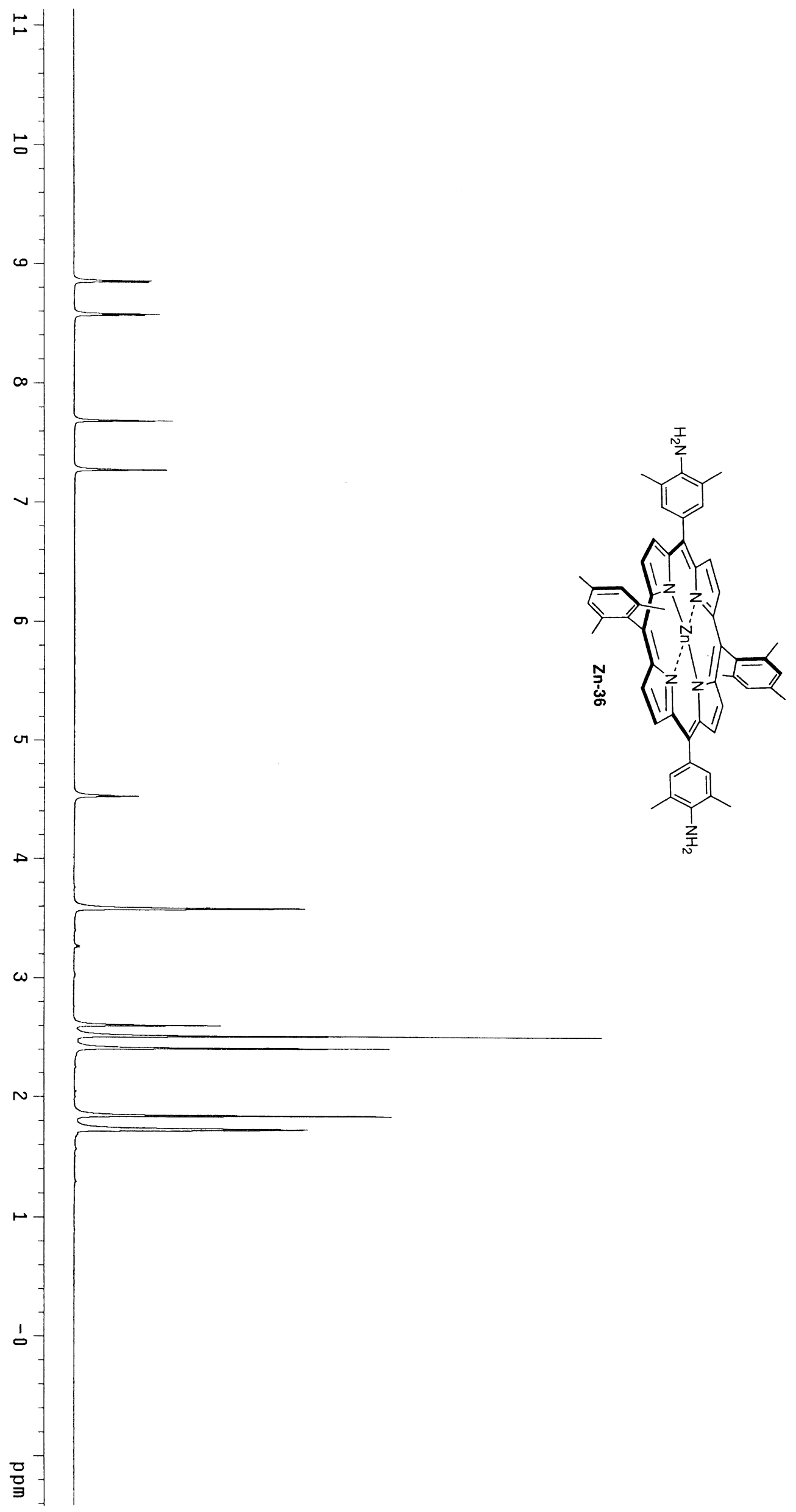




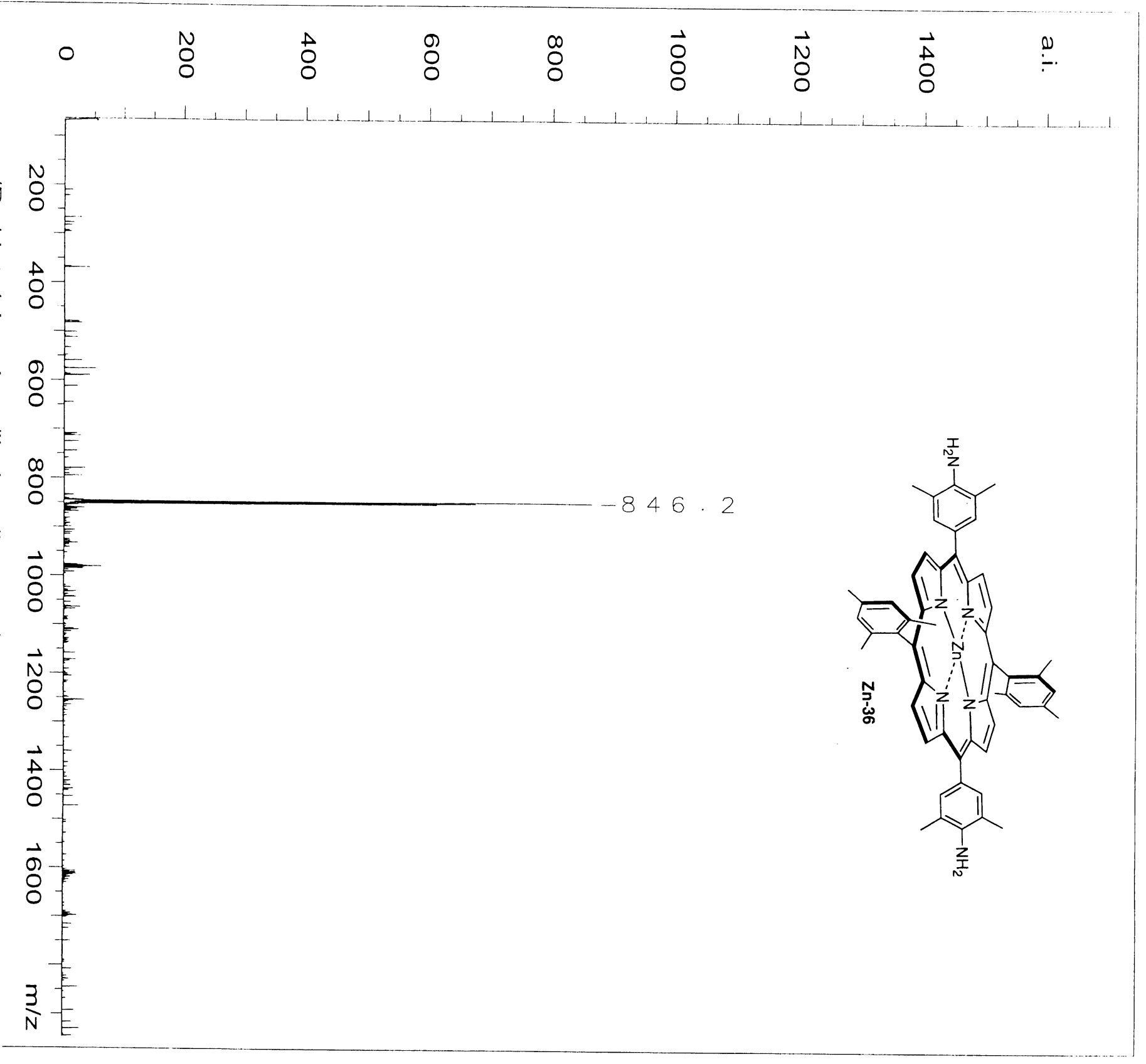

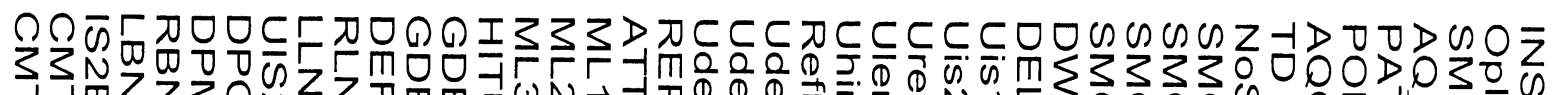

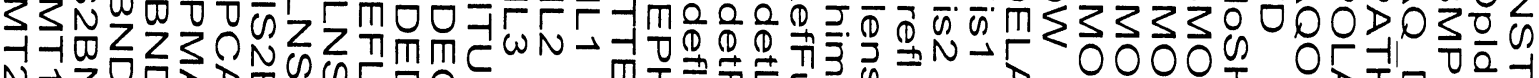

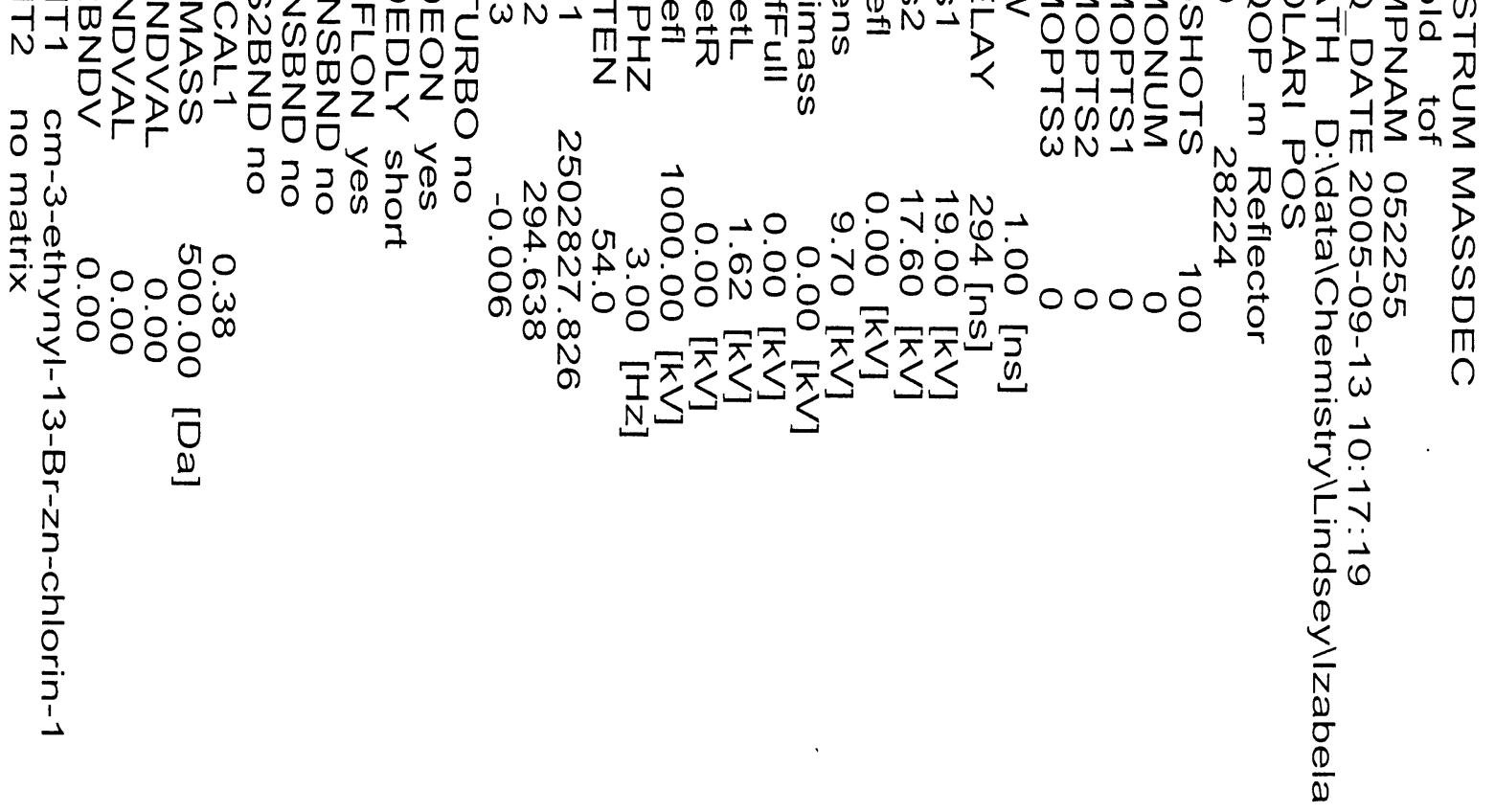




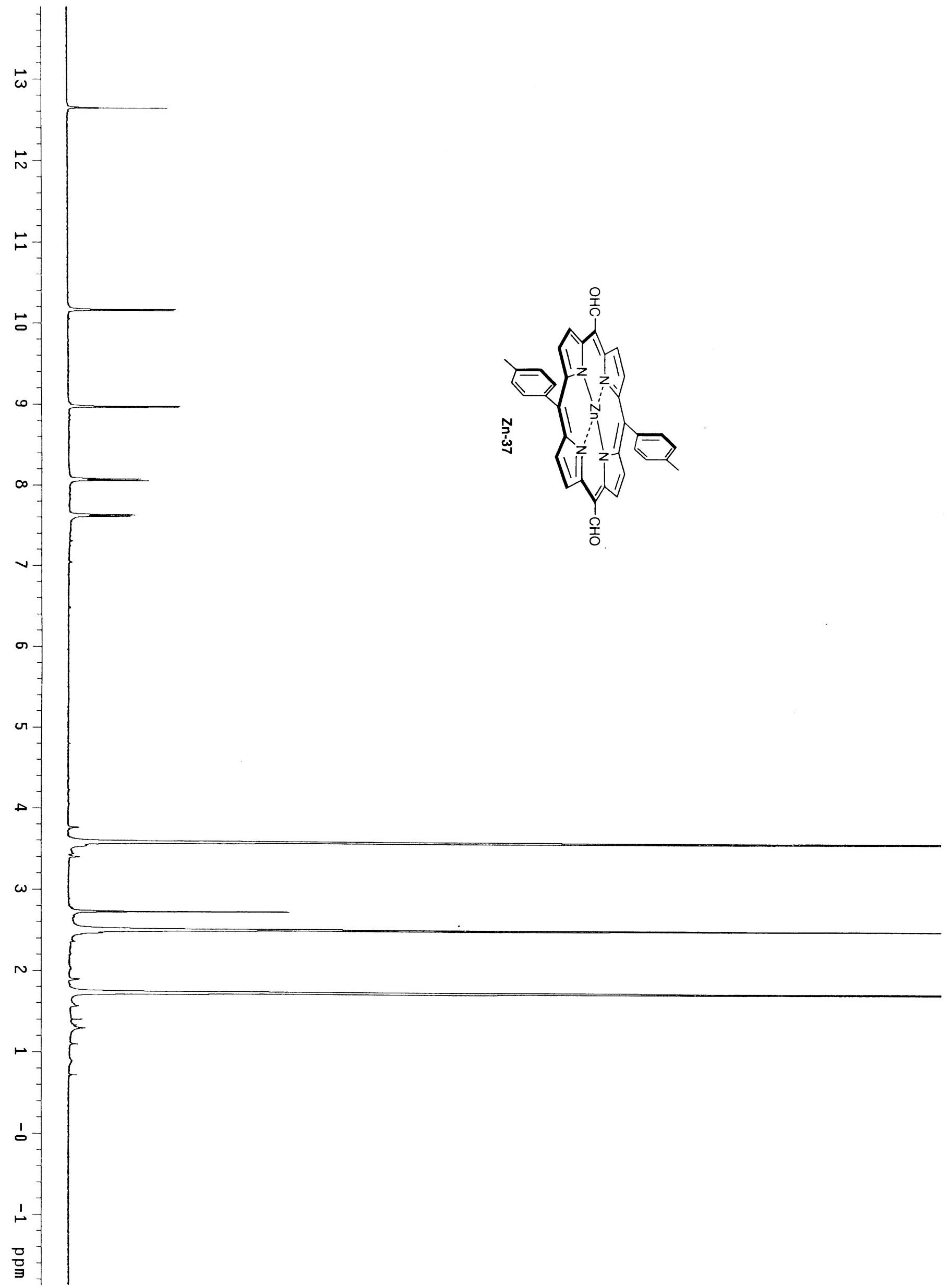


$\begin{array}{ccccccc}0 & 0 & \vec{N} & N & N & 0 \\ 0 & 0 & 0 & 0 & 0 & 0\end{array}$

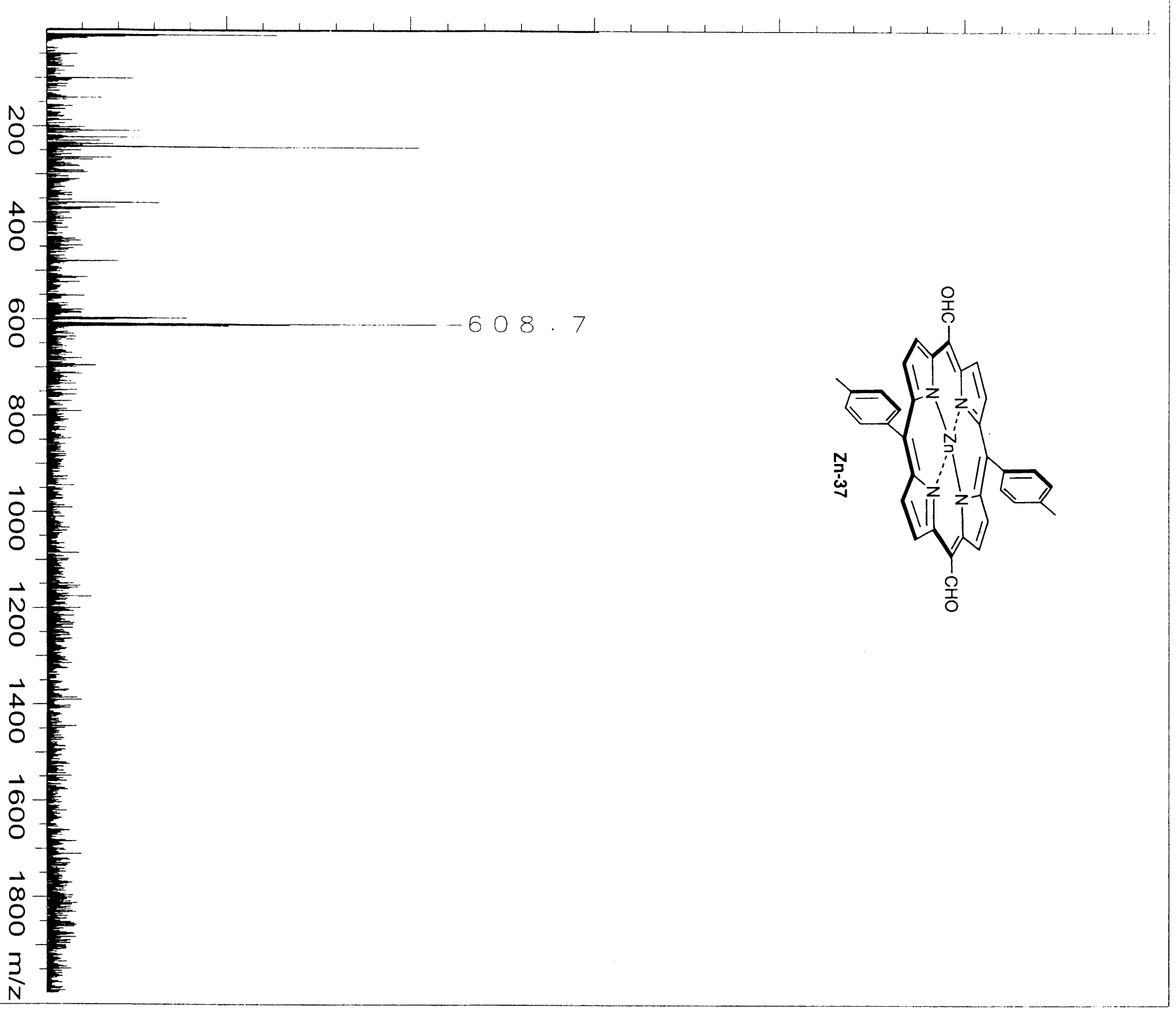

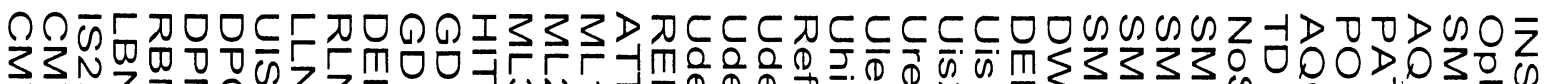

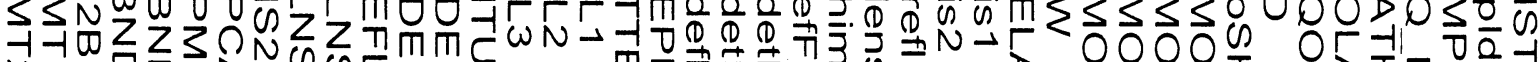

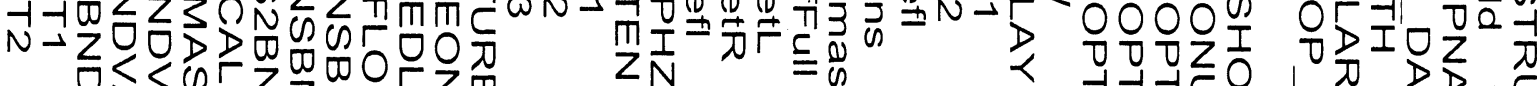

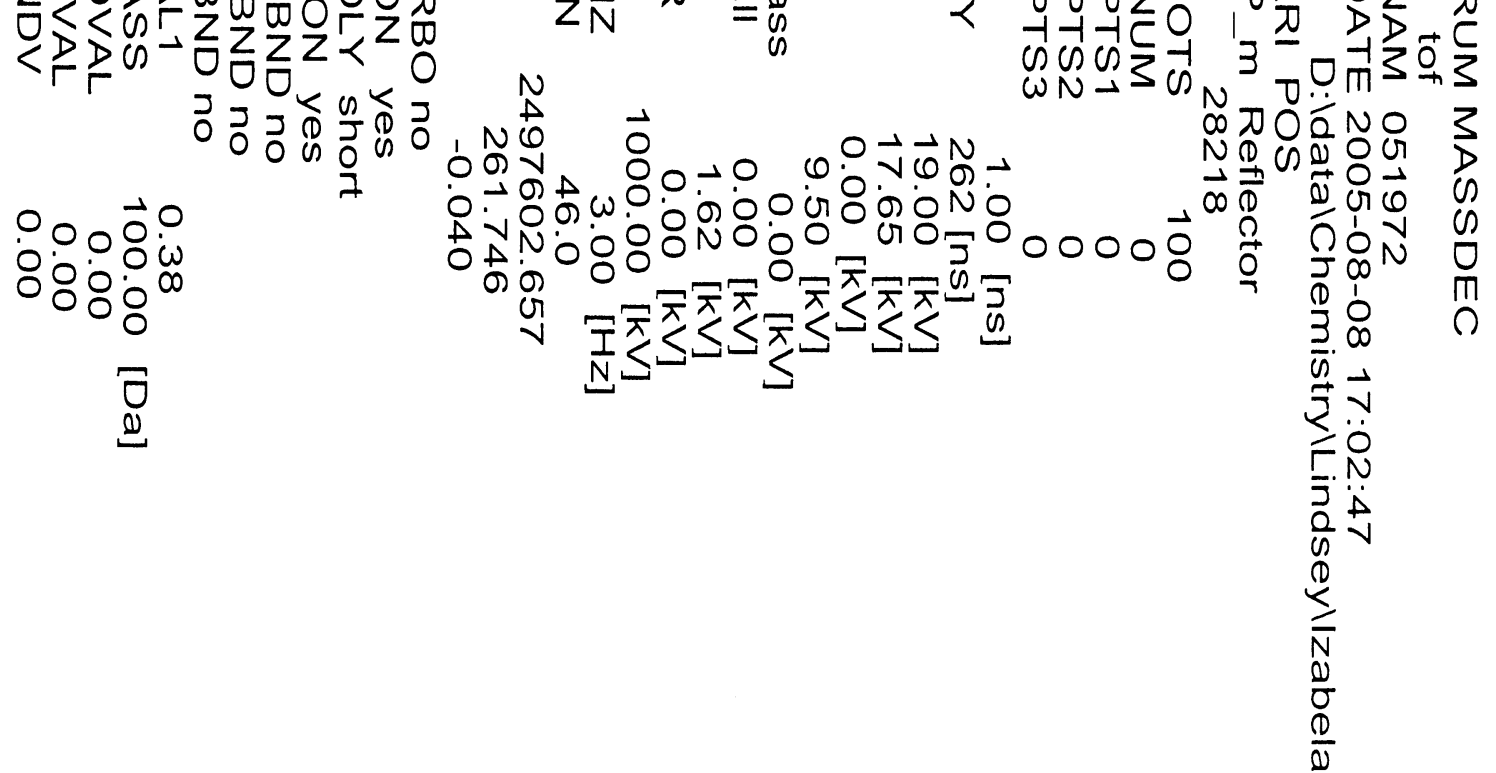



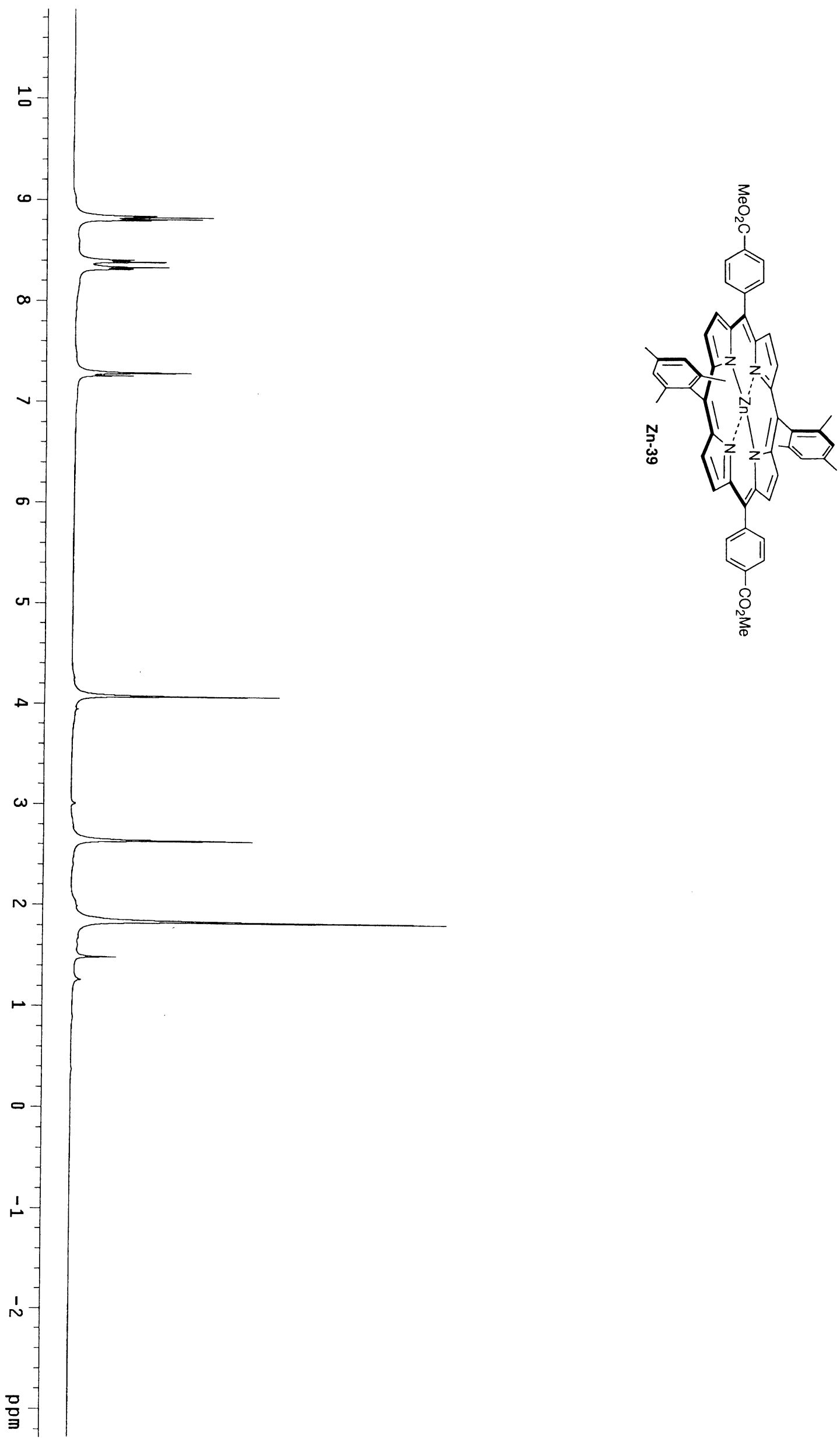
○ 芩

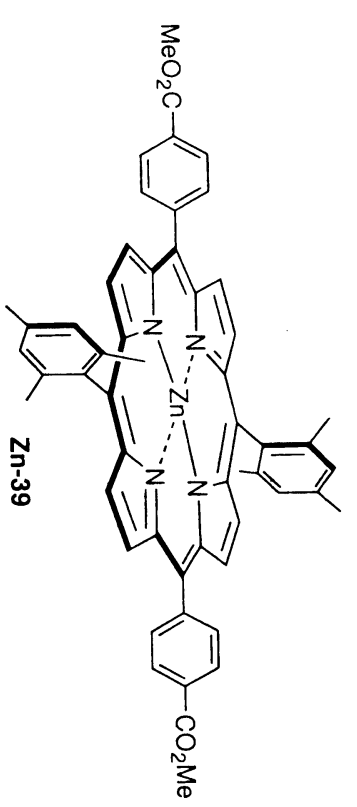

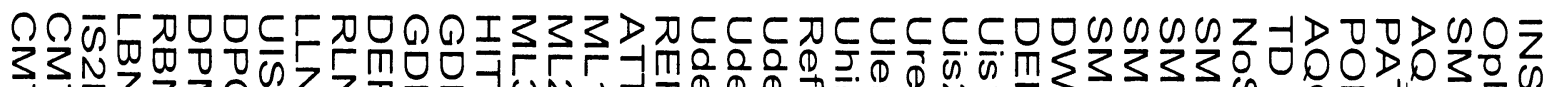
उЗN

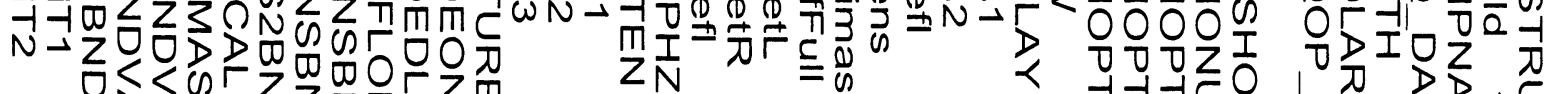

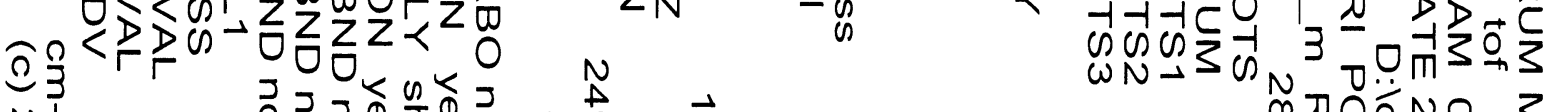

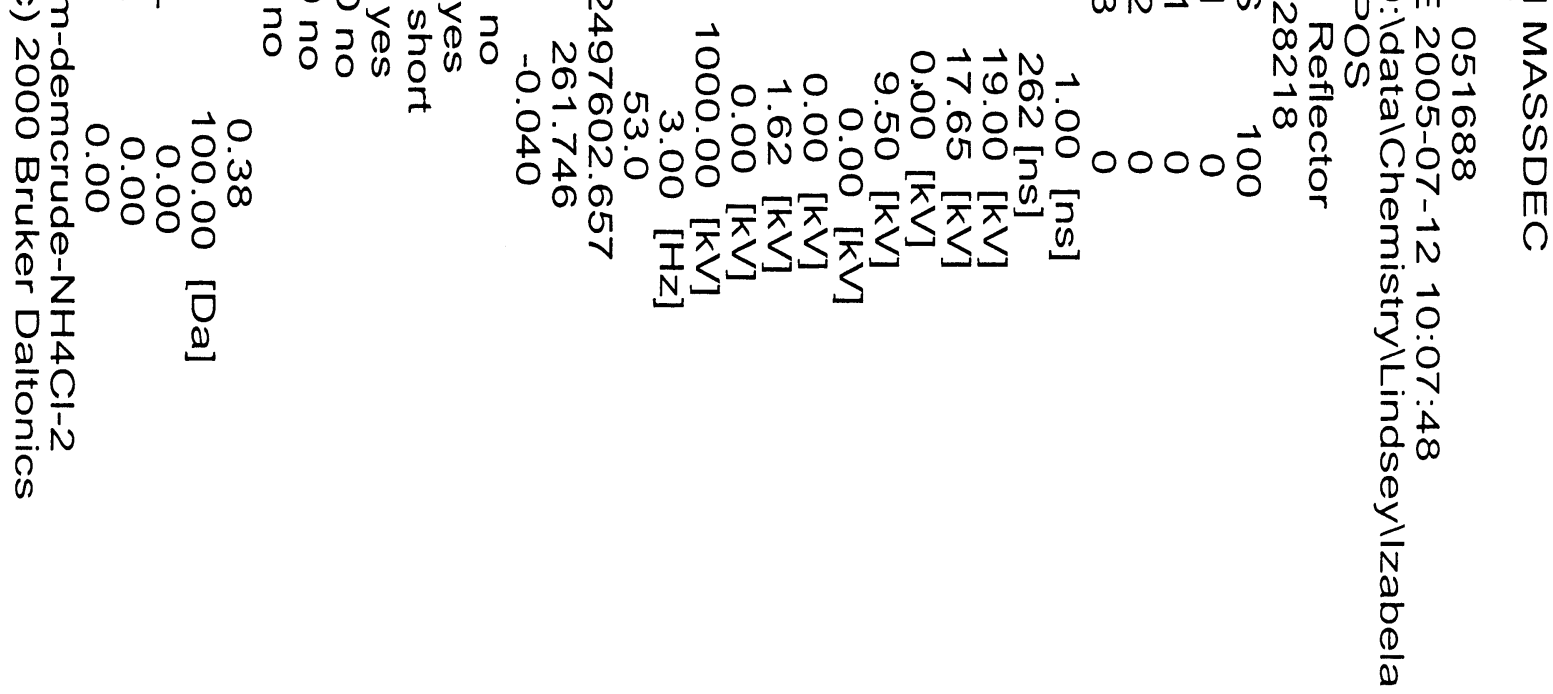



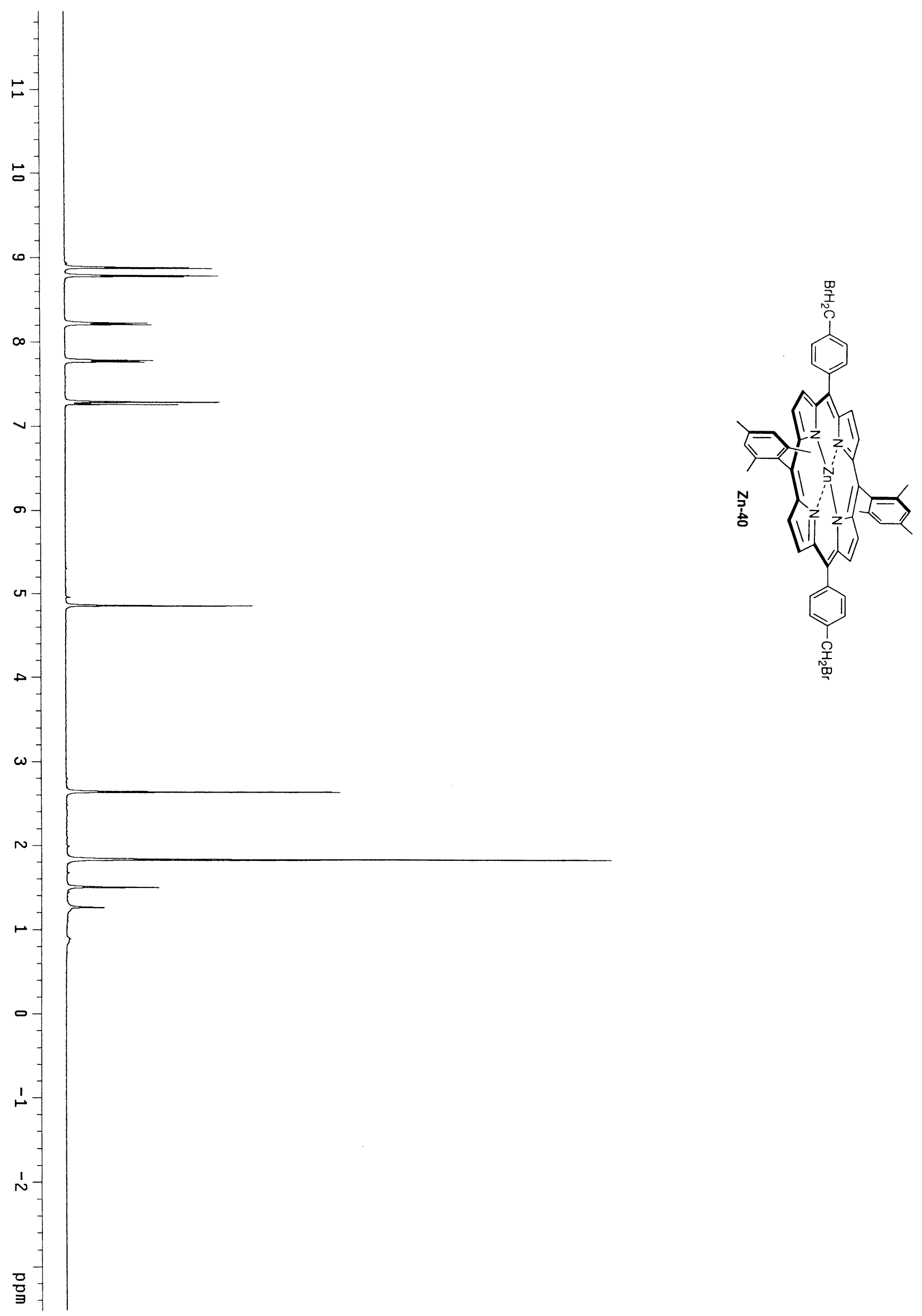


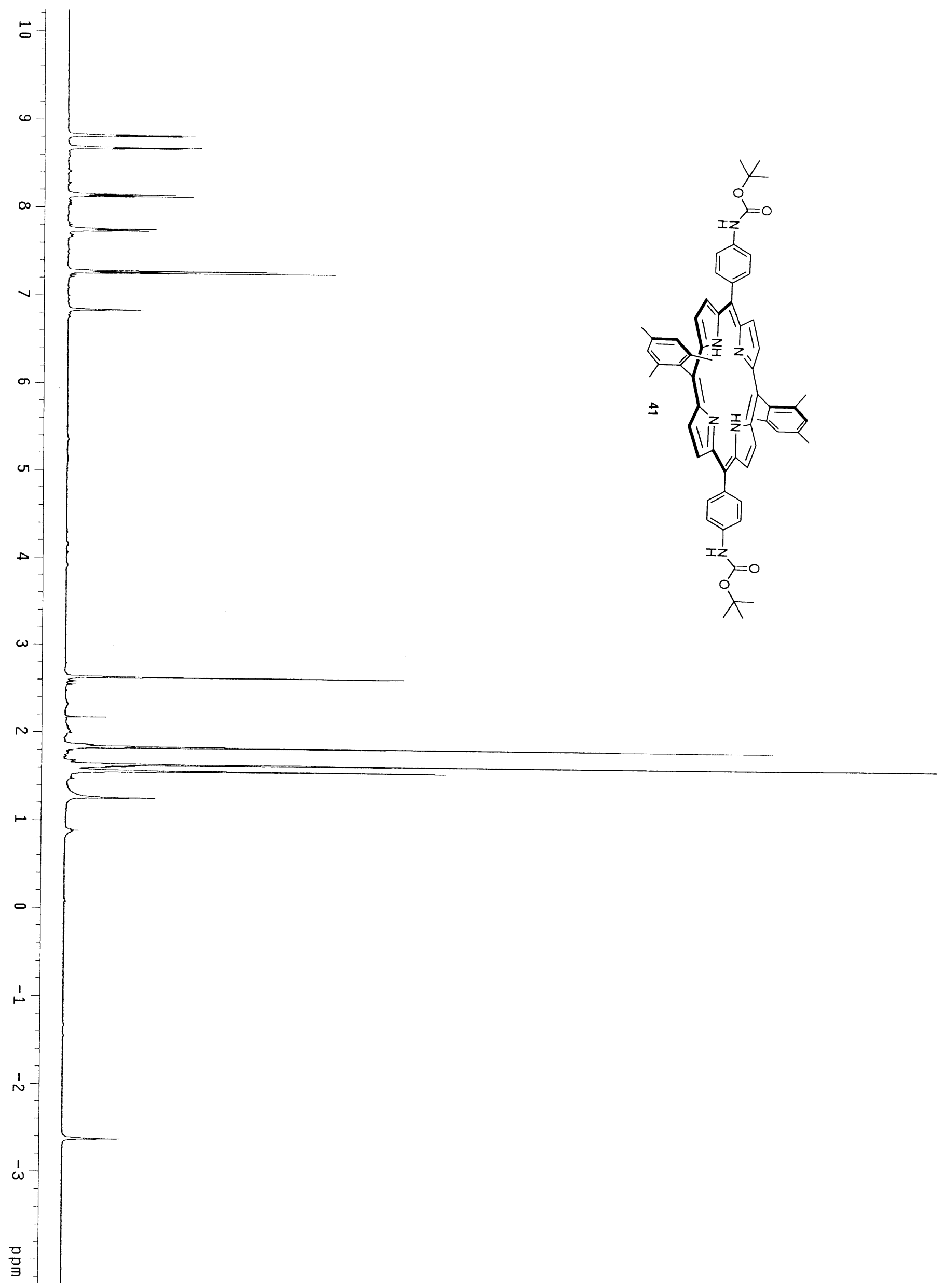


○ 省

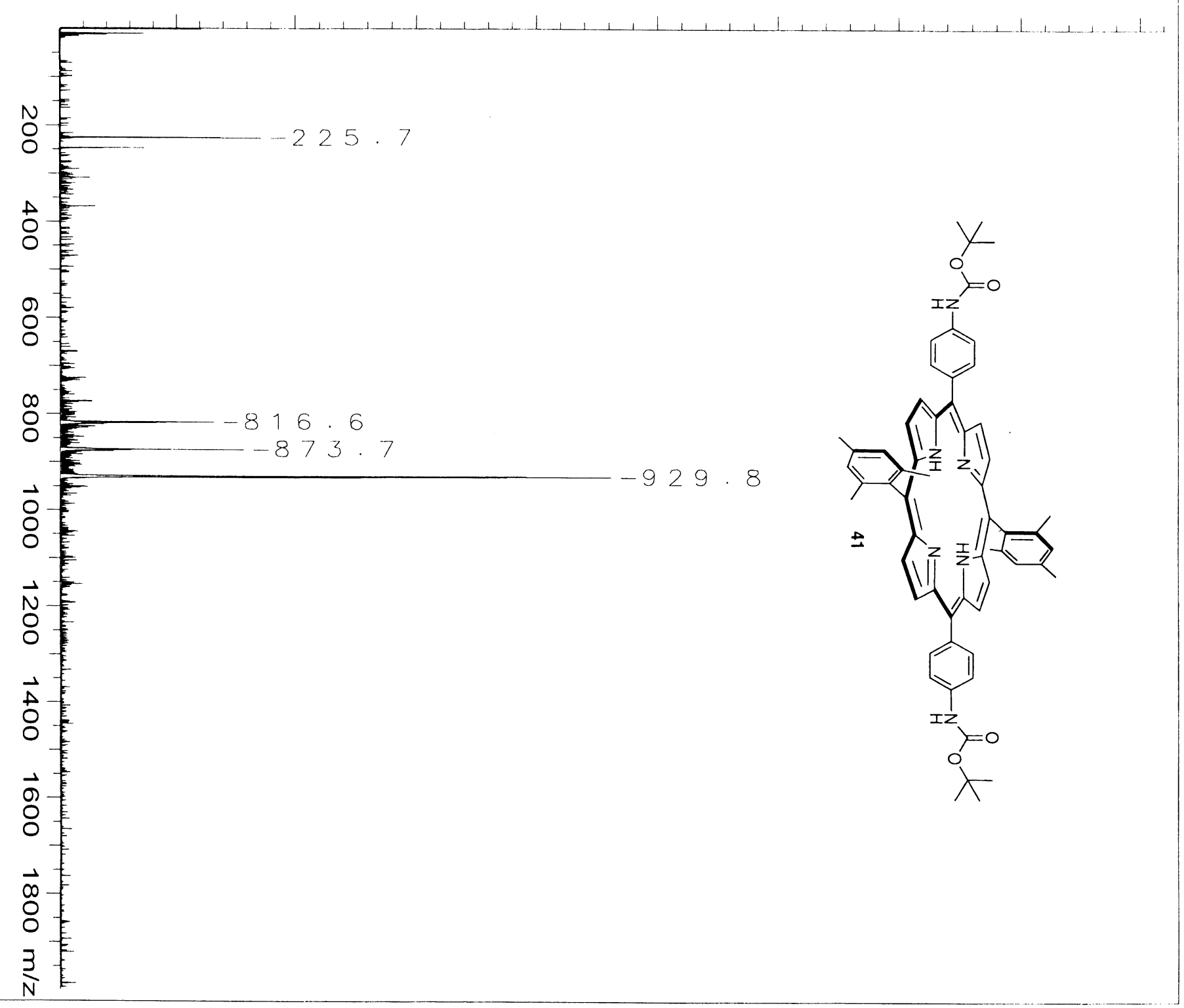

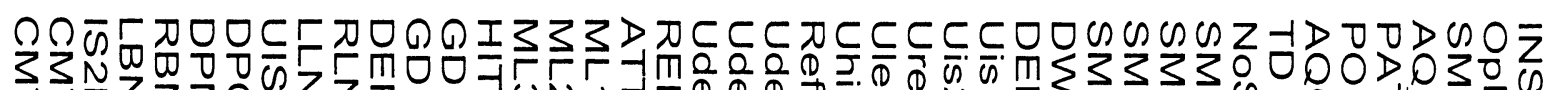

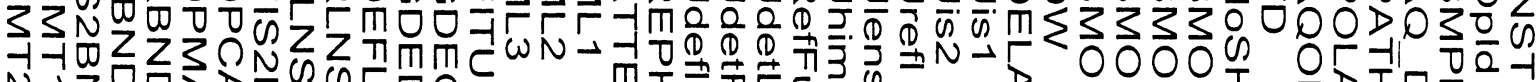

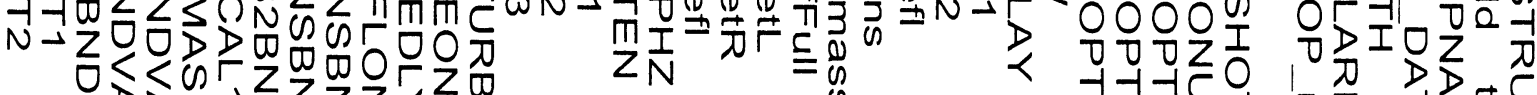

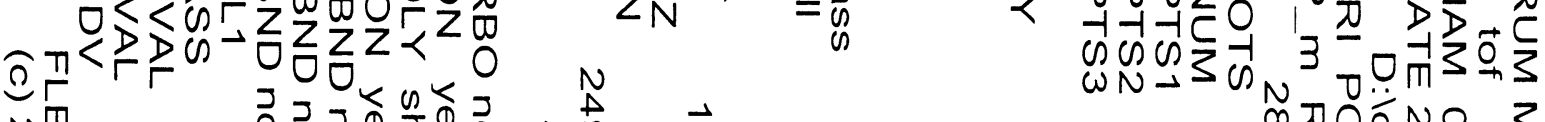

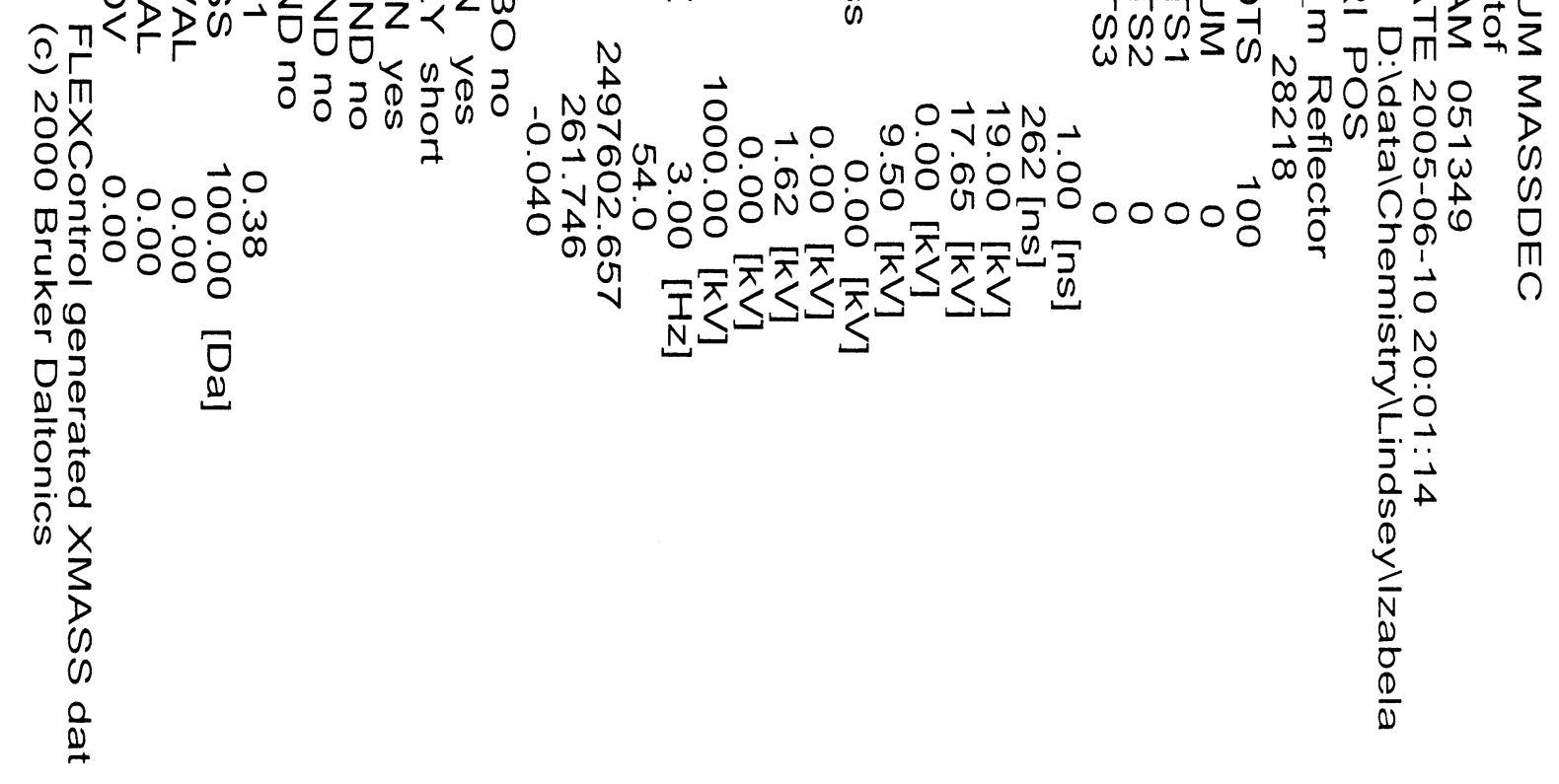



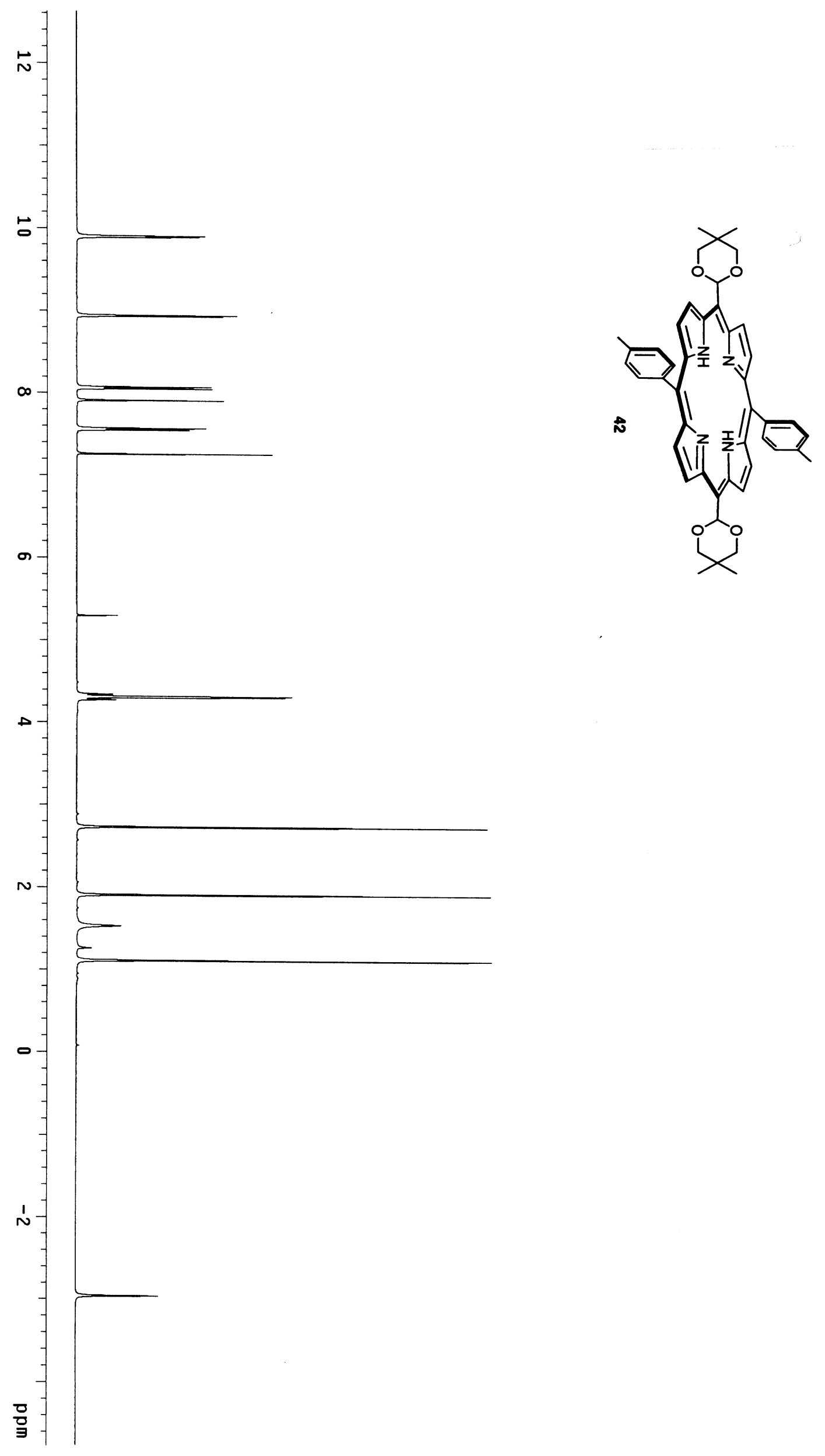


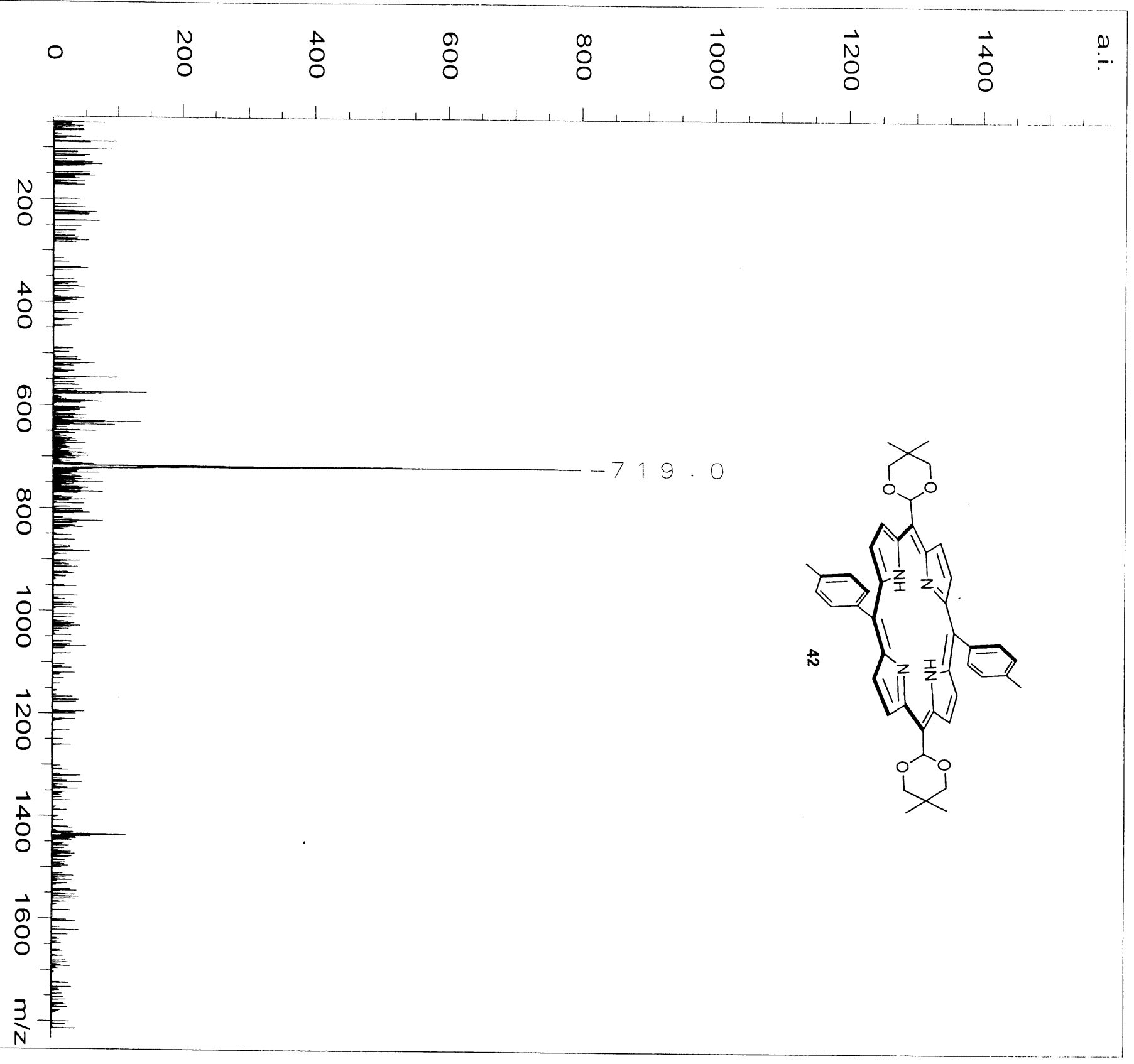

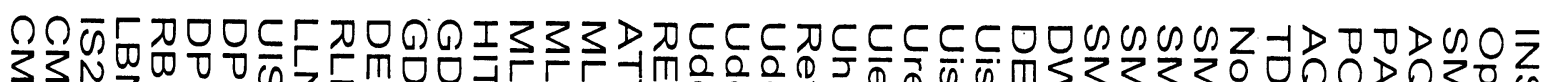

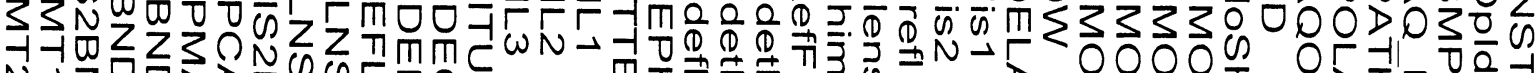

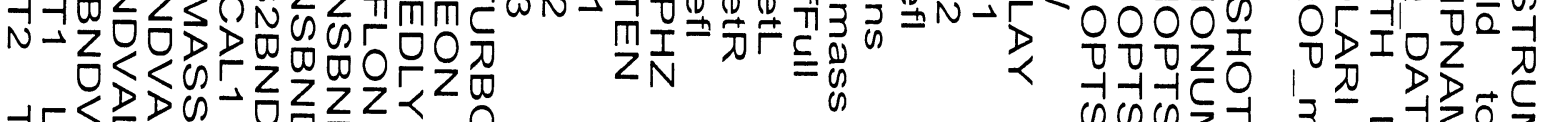

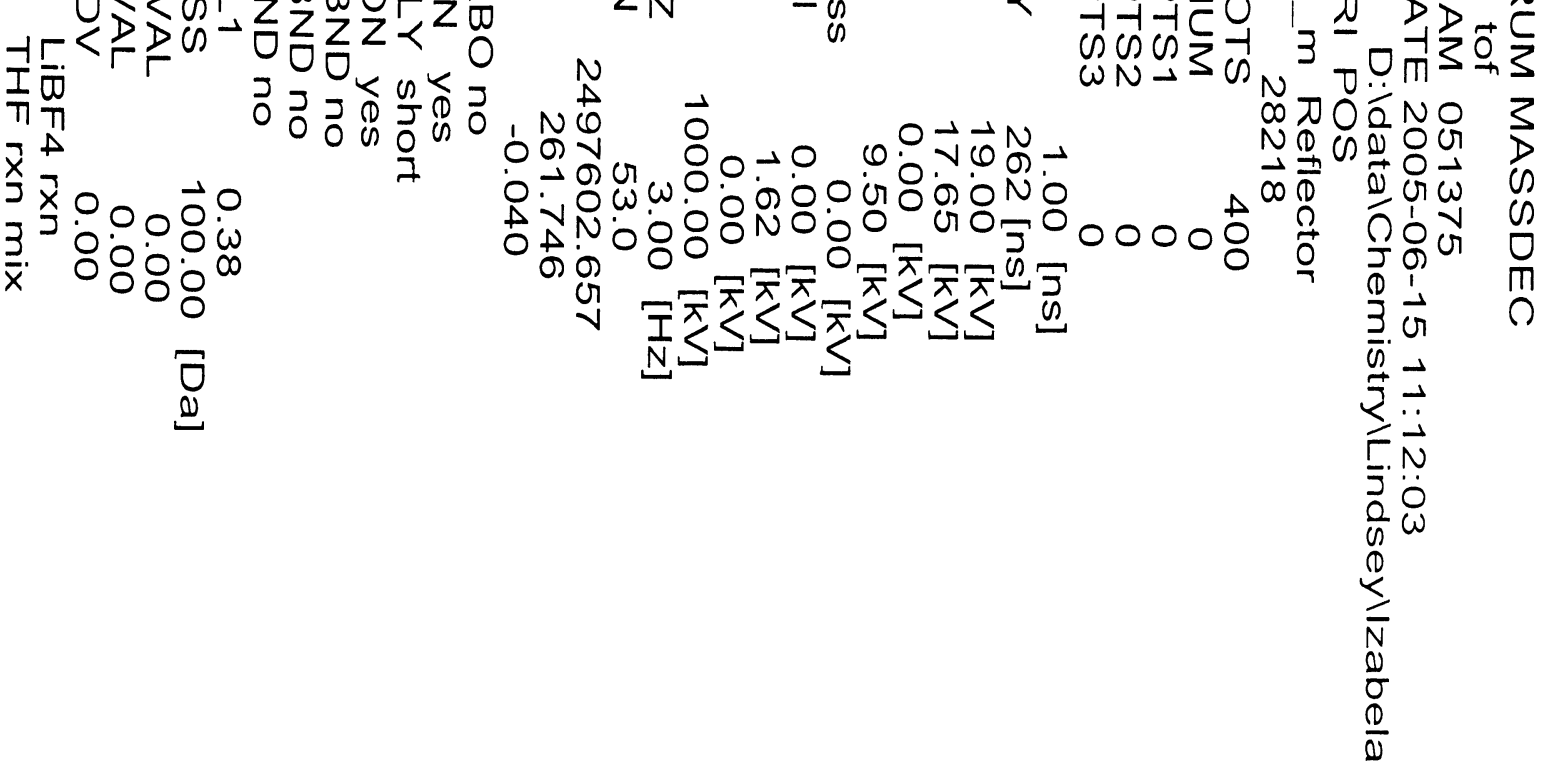




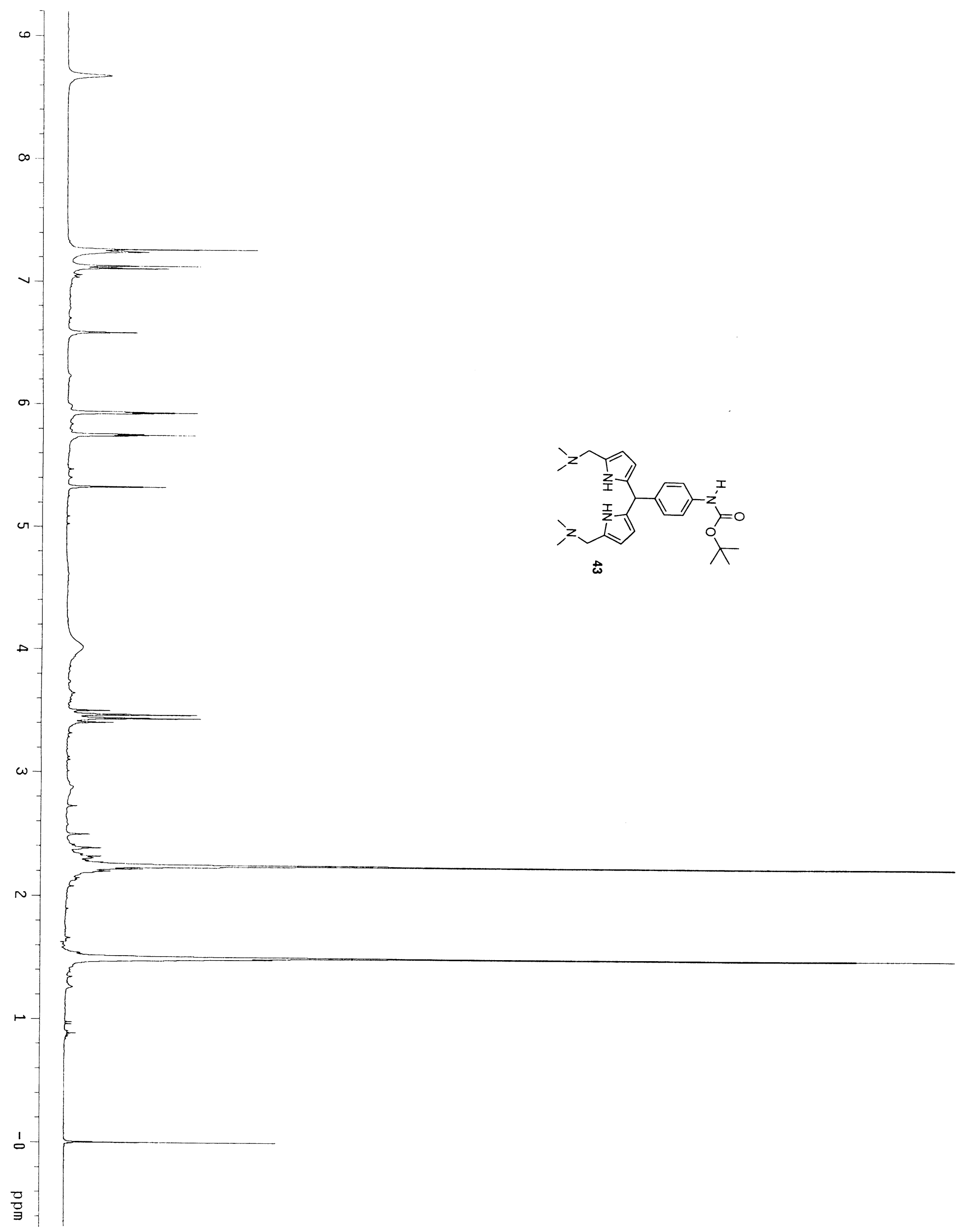




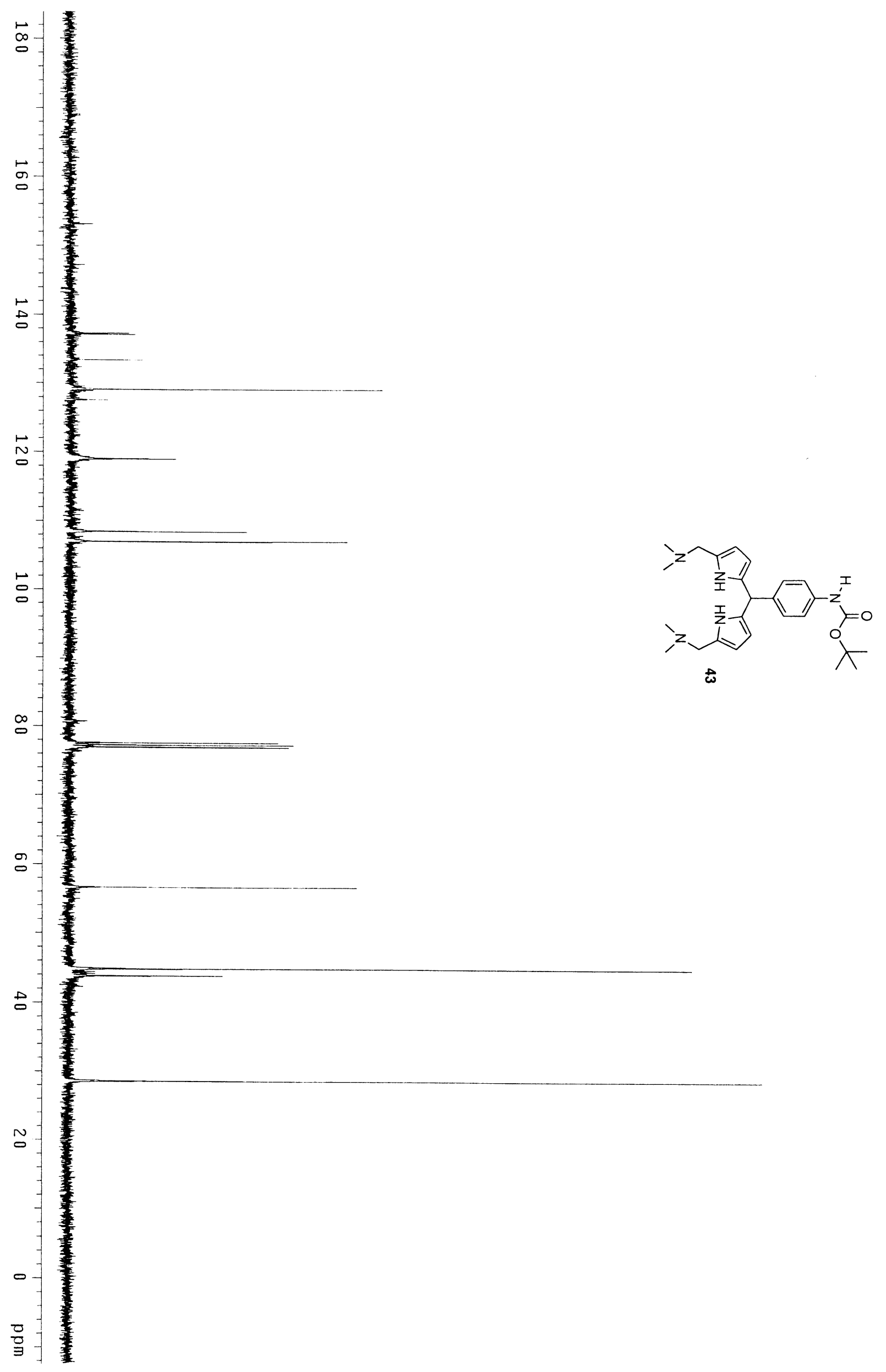




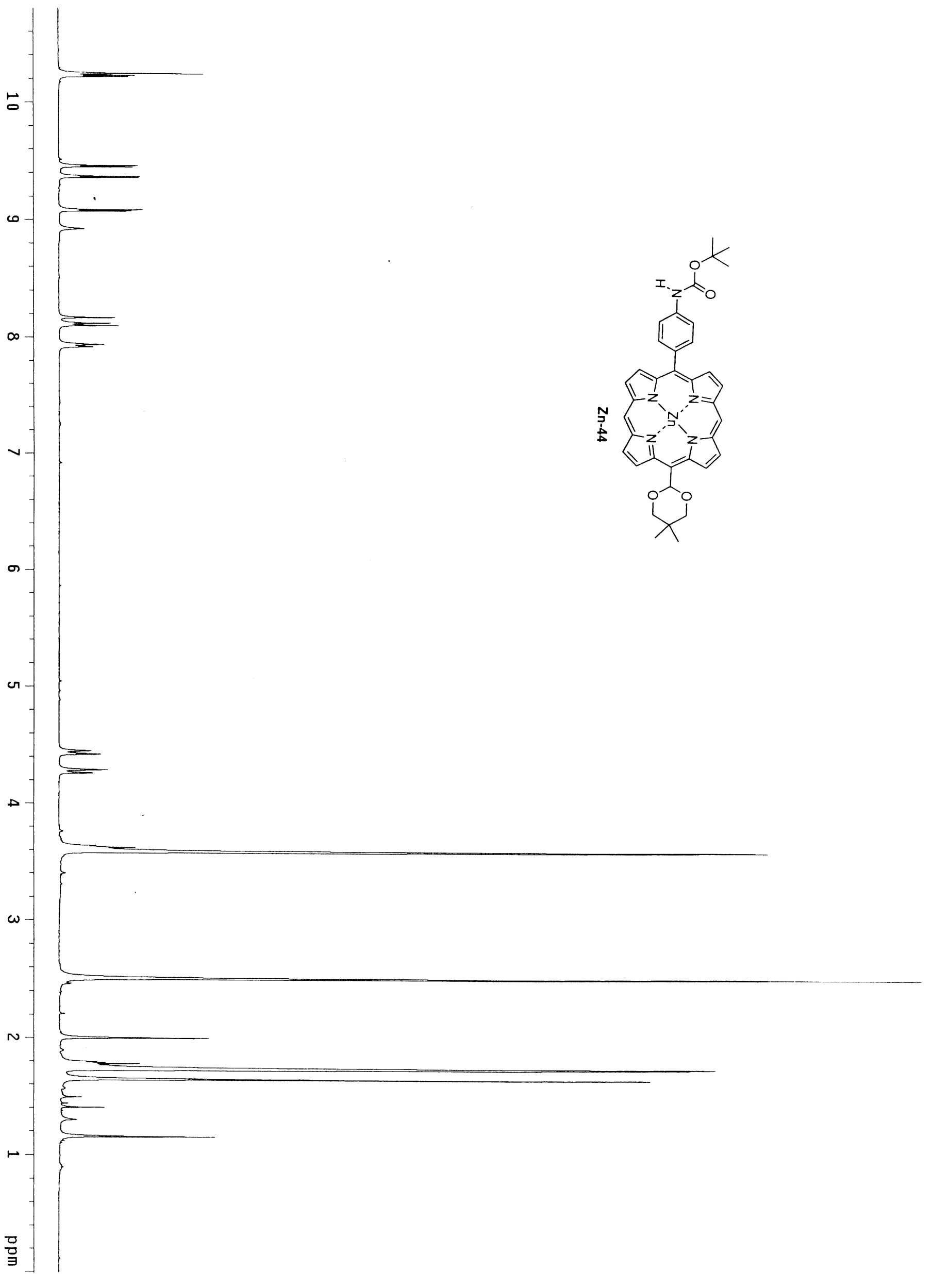



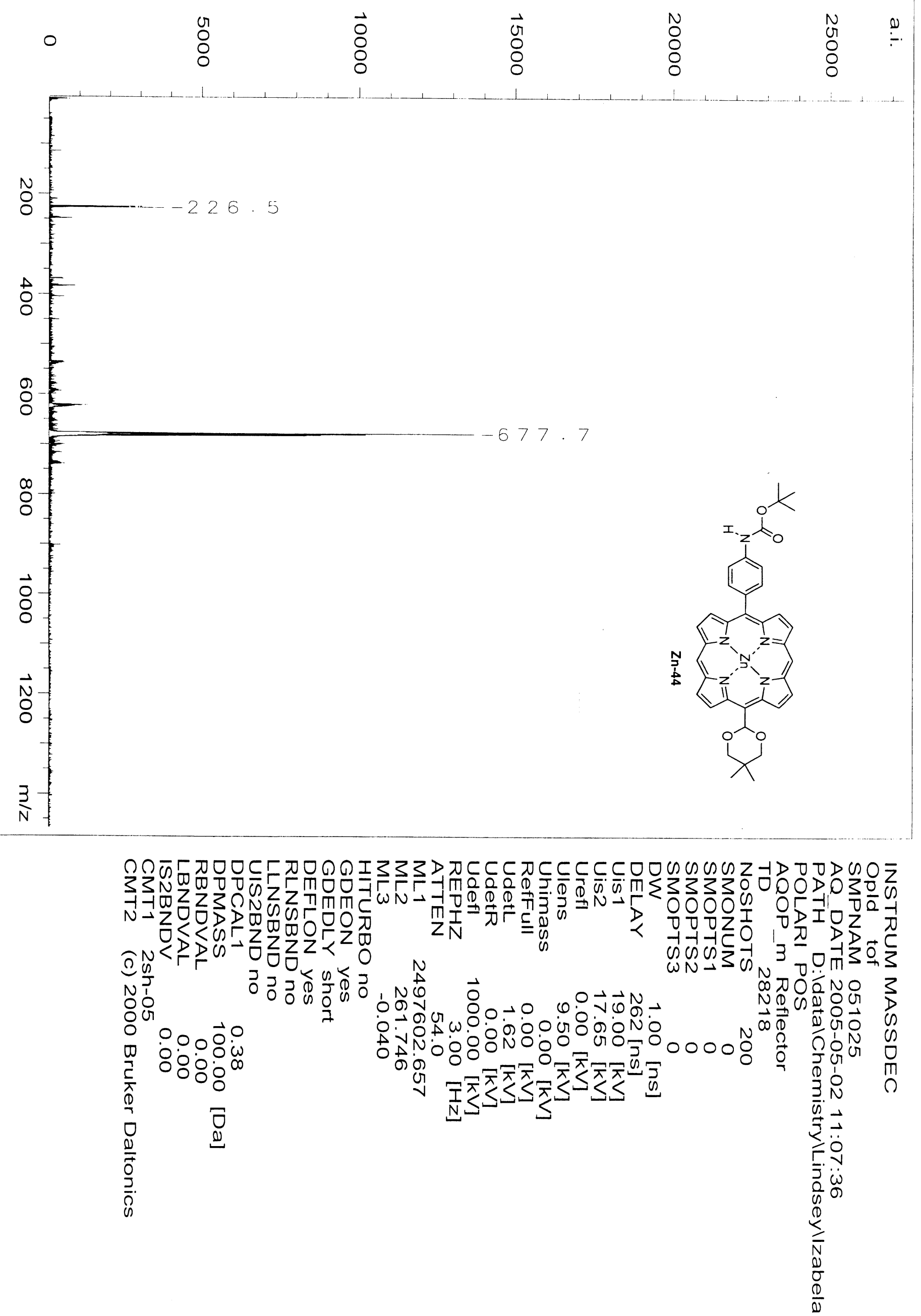


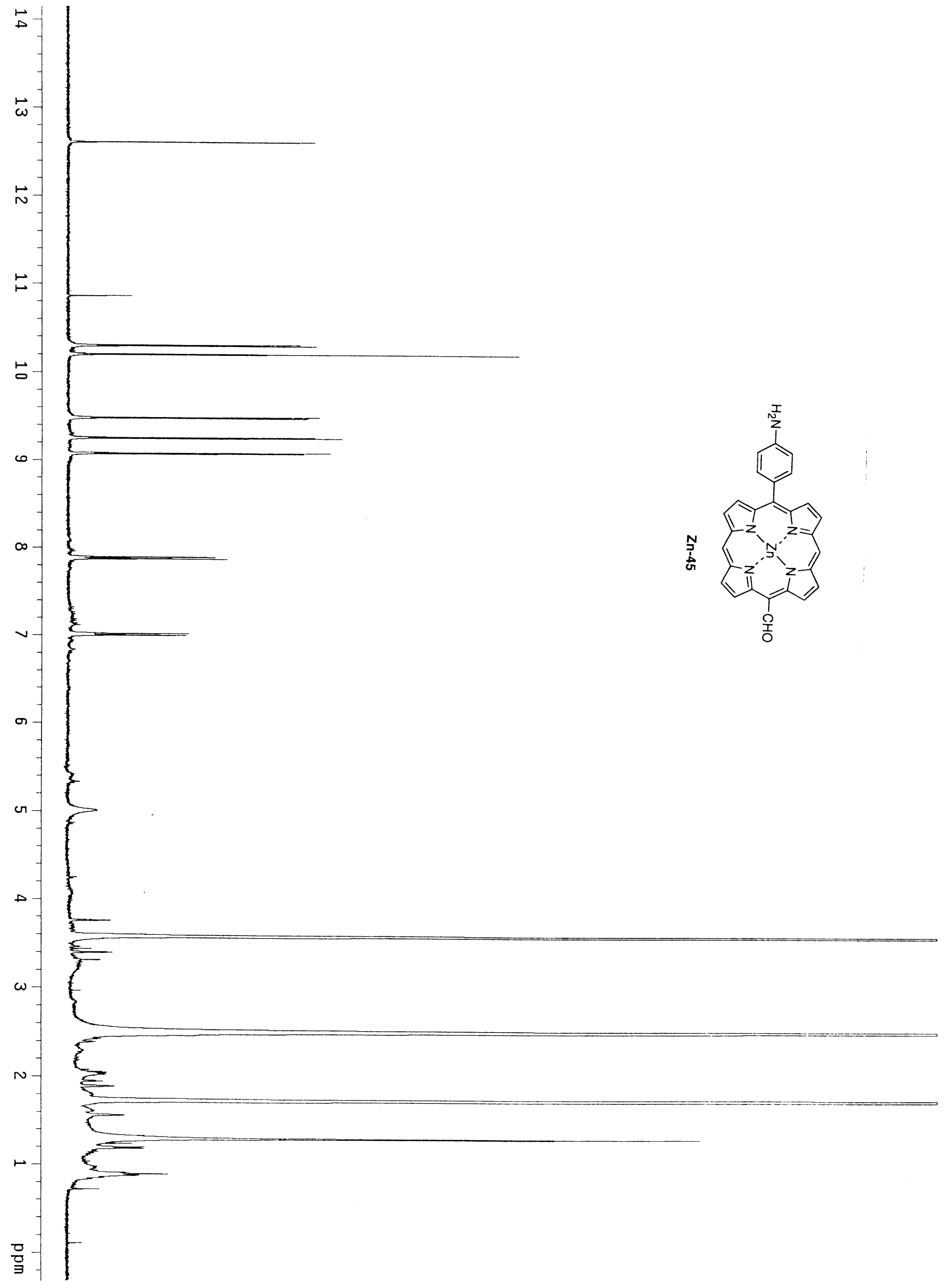



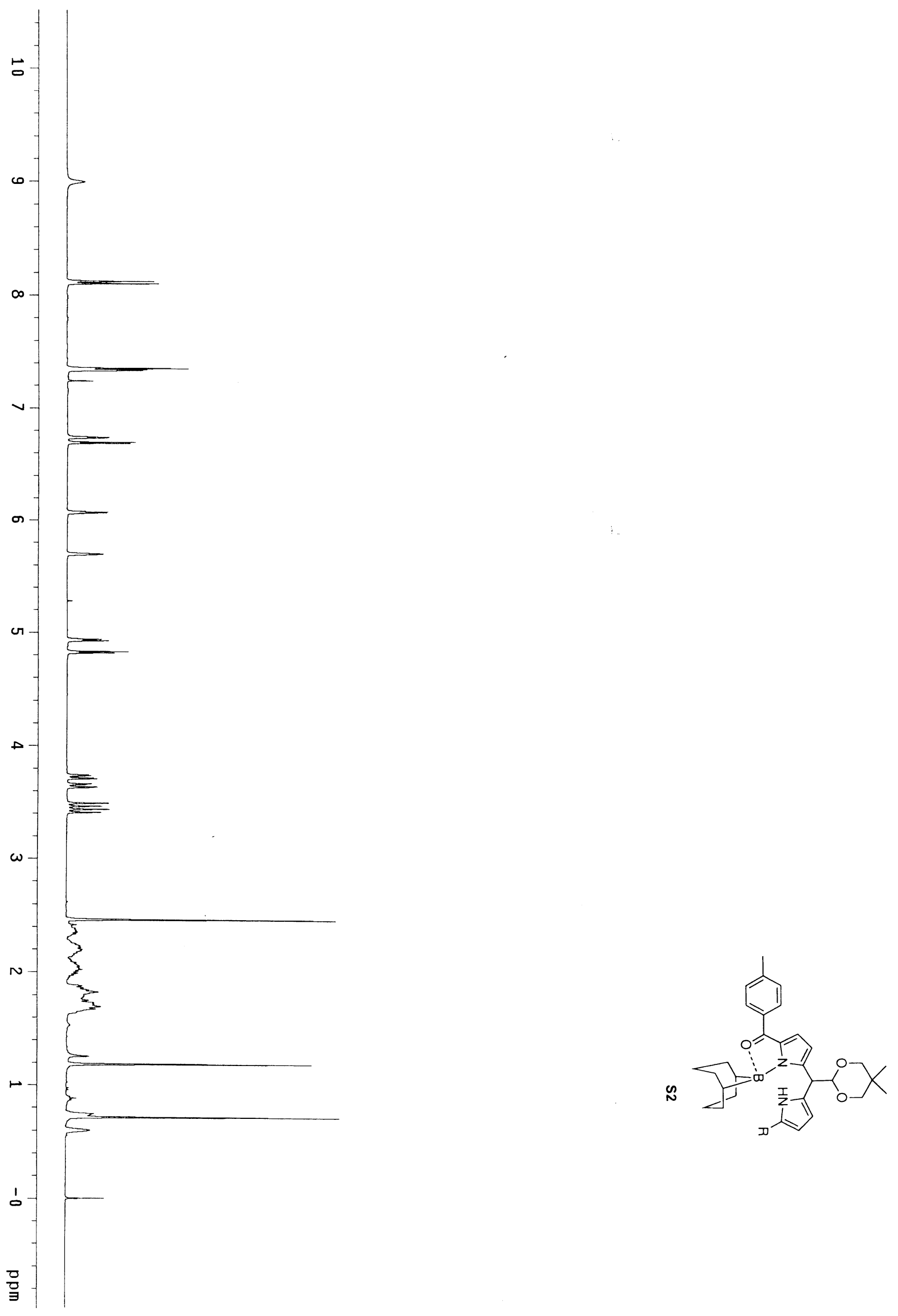

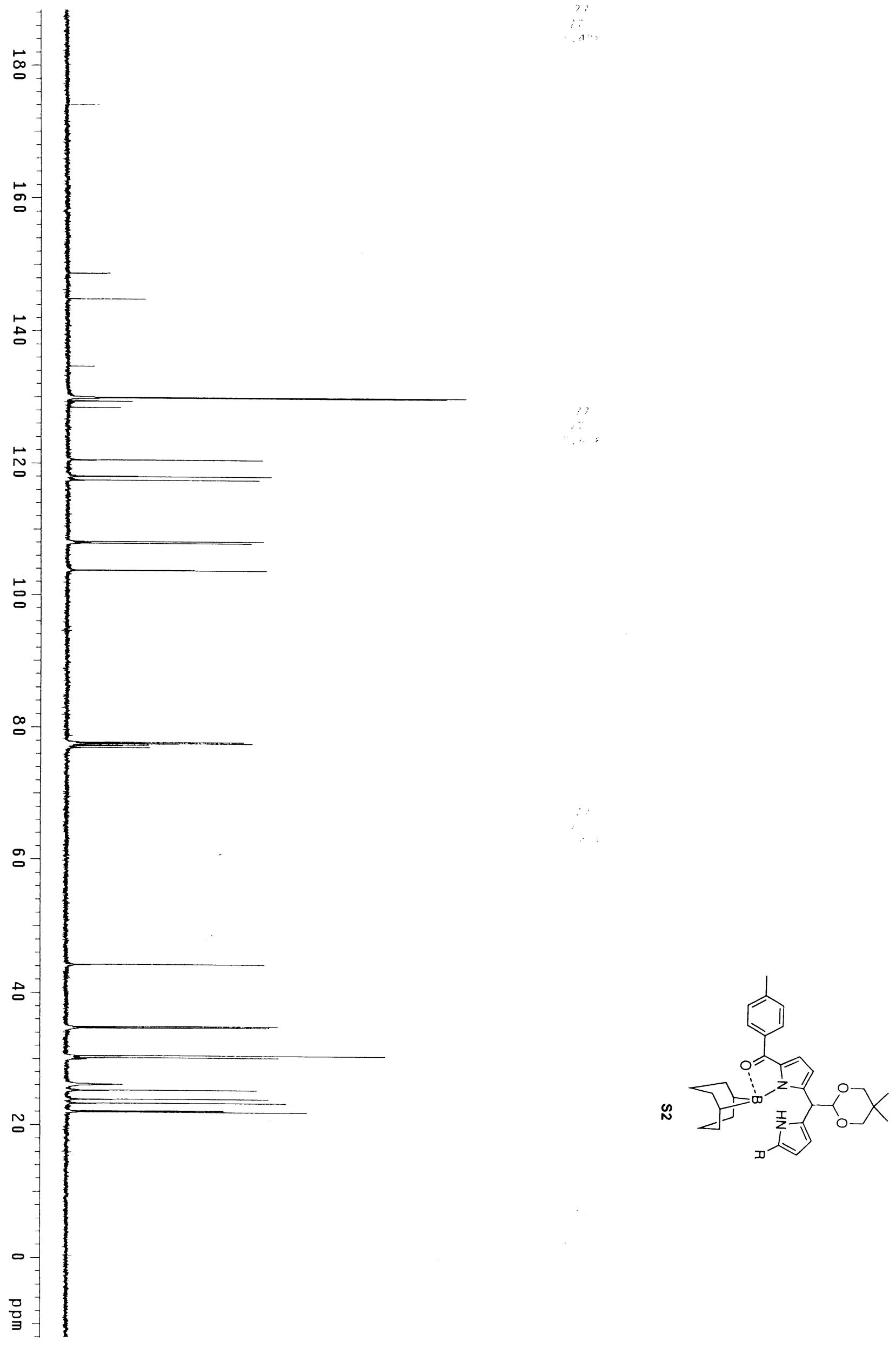


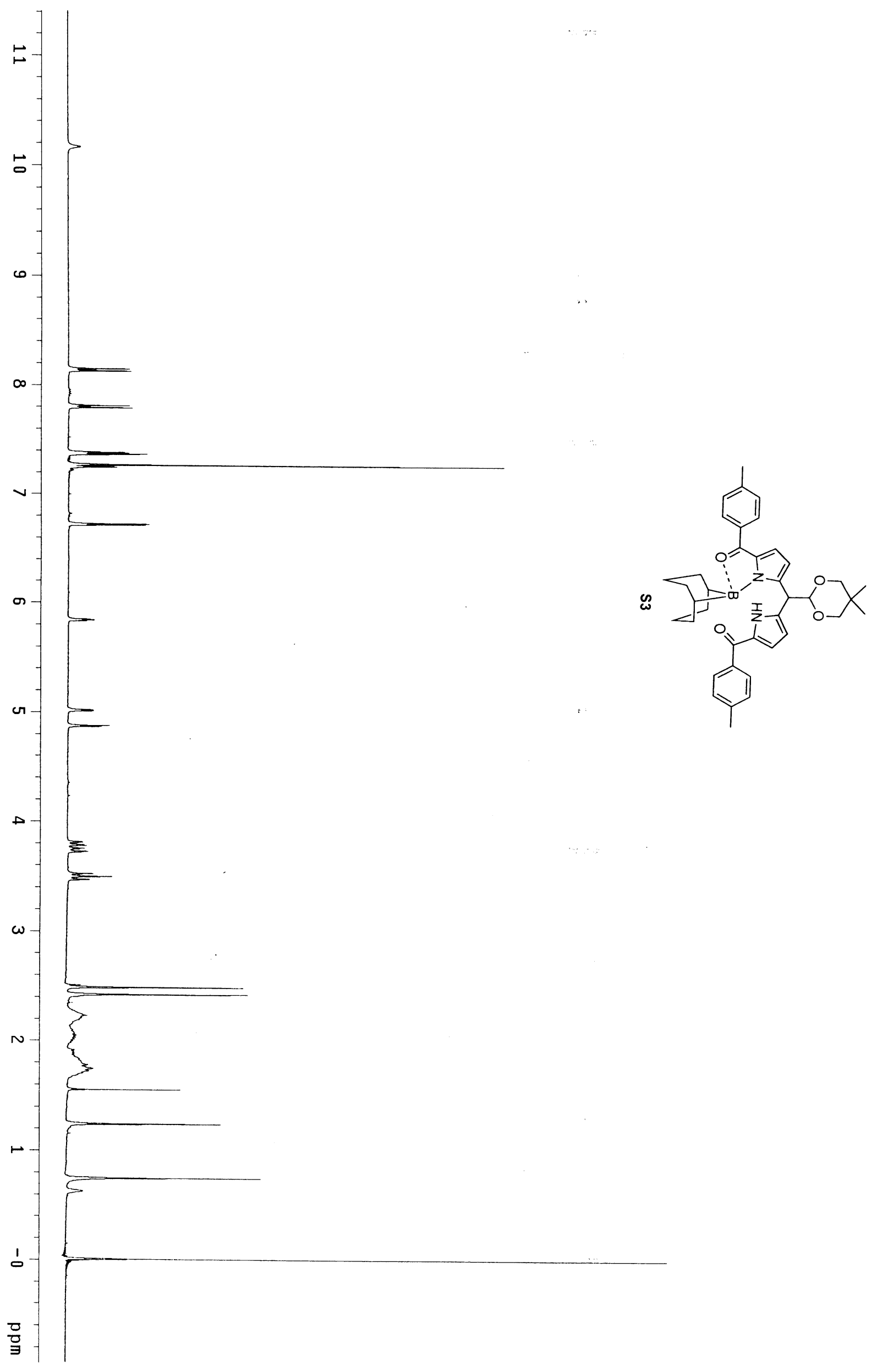




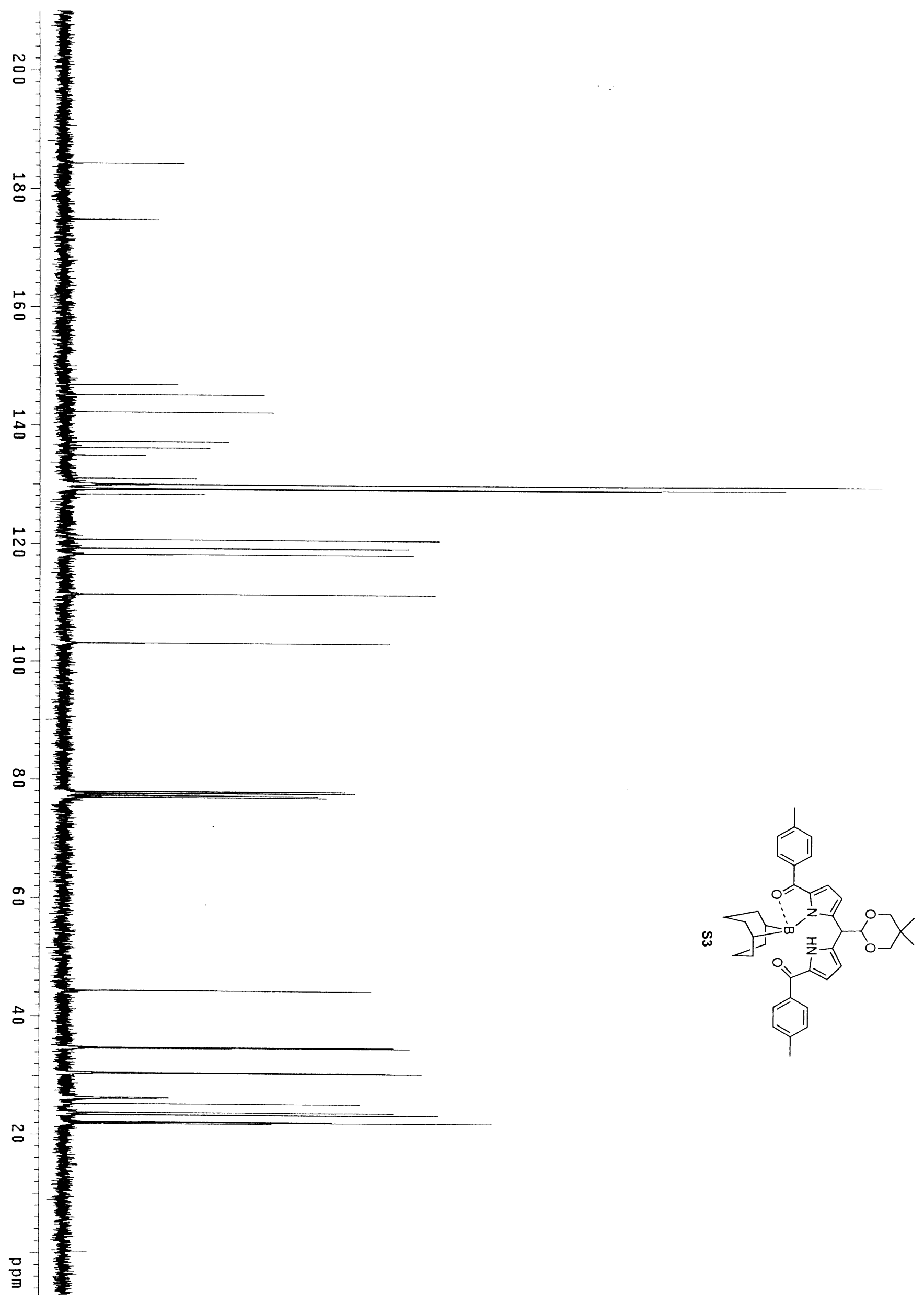

